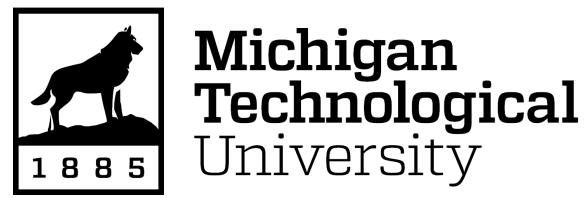

Michigan Technological University Digital Commons @ Michigan Tech

\title{
EASILY VERIFIABLE CONTROLLER DESIGN WITH APPLICATION TO AUTOMOTIVE POWERTRAINS
}

Mohammad Reza Amini

Michigan Technological University, mamini@mtu.edu

Copyright 2017 Mohammad Reza Amini

\section{Recommended Citation}

Amini, Mohammad Reza, "EASILY VERIFIABLE CONTROLLER DESIGN WITH APPLICATION TO

AUTOMOTIVE POWERTRAINS", Open Access Dissertation, Michigan Technological University, 2017.

https://doi.org/10.37099/mtu.dc.etdr/403

Follow this and additional works at: https://digitalcommons.mtu.edu/etdr

Part of the Automotive Engineering Commons, Controls and Control Theory Commons, and the Dynamics and Dynamical Systems Commons 


\title{
EASILY VERIFIABLE CONTROLLER DESIGN WITH APPLICATION TO AUTOMOTIVE POWERTRAINS
}

\author{
By \\ Mohammad Reza Amini

\begin{abstract}
A DISSERTATION
Submitted in partial fulfillment of the requirements for the degree of DOCTOR OF PHILOSOPHY
\end{abstract} \\ In Mechanical Engineering-Engineering Mechanics \\ MICHIGAN TECHNOLOGICAL UNIVERSITY
}

2017

(C) 2017 Mohammad Reza Amini 

This dissertation has been approved in partial fulfillment of the requirements for the Degree of DOCTOR OF PHILOSOPHY in Mechanical Engineering-Engineering Mechanics.

Department of Mechanical Engineering-Engineering Mechanics

Dissertation Advisor: Dr. Mahdi Shahbakhti

Committee Member: Dr. Rush D. Robinett III

Committee Member: Dr. Darrell L. Robinette

Committee Member: Dr. Jie Sun

Committee Member: Dr. Wayne W. Weaver

Department Chair: Dr. William W. Predebon 



\section{Dedication}

To:

my mother, Molouk, and my father, Jamal,

my dear wife, Sanaz,

and, Prof. J. Karl Hedrick (1944-2017) 



\section{Contents}

List of Figures $\ldots \ldots \ldots \ldots \ldots \ldots \ldots \ldots \ldots \ldots \ldots$ xiii

List of Tables ....................... $\ldots \ldots$ xxi

Preface .................... . . .

Acknowledgments . . . . . . . . . . . . . .

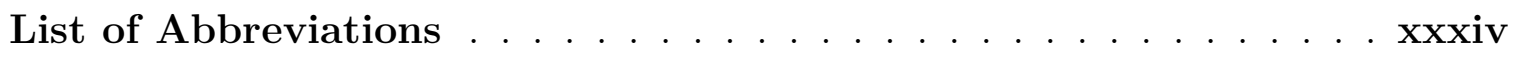

Abstract .....................

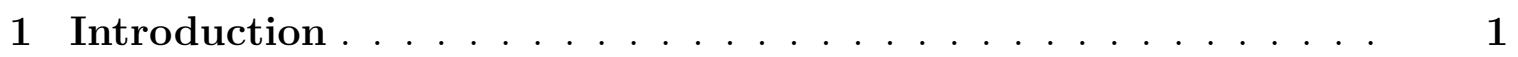

1.1 Background $\ldots \ldots \ldots \ldots \ldots \ldots \ldots \ldots \ldots \ldots \ldots$

1.2 Dissertation Objectives . . . . . . . . . . . . . . . 7

1.2.1 Dissertation Organization $\ldots \ldots \ldots \ldots$

2 Early Model-Based Design and Verification of Control Systems 13

2.1 Introduction $\ldots \ldots \ldots \ldots \ldots \ldots \ldots \ldots \ldots \ldots$

2.2 Proposed CSV Methodology . . . . . . . . . . . . . . 16 
2.2.1 Nonlinear Model Order Reduction . . . . . . . . . . . 17

2.2.1.1 Balanced realization . . . . . . . . . . 18

2.2.1.2 Singular Perturbation Approximation . . . . . . 19

2.2.2 Controller Robustness Evaluation against Implementation Imprecision ......................... 24

2.3 Case Study: Cold Start Emission Control . . . . . . . . . . . . . . 27

2.3.1 Cold start emission model . . . . . . . . . . . . 27

2.3.2 Reduced Order Engine Model/Controller . . . . . . . . . . 29

2.3.3 Controller Design ................. 34

2.3.4 HC Emission Control Results .............. 37

2.3.4.1 Robustness to Implementation Imprecision . . . . . 41

2.4 Summary and Conclusion ................. 43

3 Easily Verifiable Continuous-Time Controller Design . . . . . . . 45

3.1 Introduction . . . . . . . . . . . . . . . . 45

3.2 Uncertainty Adaptive SMC Robust to Implementation Imprecisions 47

3.3 Case Study: Cold Start Emission Control . . . . . . . . . . . . . 51

3.3.1 Baseline SISO Sliding Controller Design \& Impact of Implementation Imprecisions . . . . . . . . . . . . . 52

3.3.2 Uncertainty-Adaptive SISO Sliding Controller Design . . . . 56

3.3.3 MIMO Uncertainty-Adaptive SMC Design . . . . . . . . . 59

3.3.4 Real-Time Controller Verification ........... 62 
3.4 Summary and Conclusion ................. 69 69

4 Discrete SMC with Robustness to Implementation Imprecisions 71

4.1 Introduction . . . . . . . . . . . . . . . . . 71

4.2 DSMC with Predicted Uncertainties . . . . . . . . . . . 73

4.2.1 Uncertainty Prediction on Measured Signal Due to ADC . . 73

4.2.2 DSMC Design and Uncertainty Propagation . . . . . . . 75

4.2.3 Inclusion of Propagated Uncertainties into the DSMC . . . 80

4.3 Case Studies . . . . . . . . . . . . . . . . . . . 81

4.3.1 Linear Case Study: DC Motor Speed Control . . . . . . . 81

4.3.2 Nonlinear Case Study: Automotive Engine Control . . . . . 85

4.4 DSMC Real-Time Verification . . . . . . . . . . . . . . 91

4.5 Summary and Conclusion . . . . . . . . . . . . . 91

$5 \quad$ First Order SISO Adaptive Discrete Sliding Mode Control . . 93

5.1 Introduction . . . . . . . . . . . . . . . . 93

5.2 Adaptation Against Model Uncertainties . . . . . . . . . . . . . . 94

5.3 Nonlinear Case Study: Engine Control . . . . . . . . . . . . . . 100

5.4 First Order Adaptive DSMC for Linear Uncertain Systems . . . . . 117

5.4.1 Linear Case Study: DC Motor Speed Control . . . . . . . . 125

5.4.2 DC Motor First Order Adaptive DSMC Real-Time Verification $\quad 126$

5.5 Summary and Conclusion . . . . . . . . . . . . 128 
6 Second Order SISO/MIMO Adaptive DSMC . . . . . . . . . 131

6.1 Introduction . . . . . . . . . . . . . . . . . . 131

6.2 SISO Second Order Sliding Mode Control . . . . . . . . . . 134

6.2.1 Continuous-Time Second Order SMC . . . . . . . . . 134

6.2.2 Discrete-Time Adaptive Second Order SMC . . . . . . . 136

6.3 MIMO Adaptive Second Order DSMC $\ldots \ldots \ldots \ldots$

6.4 Nonlinear Case Study: Engine Control . . . . . . . . . . . . 158

6.4.1 Handling Implementation Imprecisions $\ldots \ldots \ldots$

6.4.2 External Disturbance Rejection . . . . . . . . . . . 164

6.4.3 Real-Time Engine $2^{\text {nd }}$ Order DSMC Verification . . . . . 168

6.5 Second Order Adaptive DSMC for Uncertain Linear Systems . . . 172

6.5.1 Linear Case Study: DC Motor Speed Control . . . . . . . 173

6.5.2 Real-Time DC motor $2^{\text {nd }}$ order DSMC Verification $\ldots \ldots$

6.6 Summary and Conclusion $\ldots \ldots \ldots \ldots$

7 Experimental Testing of the DSMC on an Electronic Throttle

Body .......................... 181

7.1 Introduction . . . . . . . . . . . . . . . . . . 181

7.2 Throttle Experimental Setup . . . . . . . . . . . . . . . . 184

7.3 Throttle Modelling and Parameter Identification . . . . . . . . . 185

7.4 DSMC design for Throttle Position Control . . . . . . . . 193

7.5 Second Order DSMC for Throttle Position Control . . . . . . . 201 
7.6 Summary and Conclusion ................ 204

8 Conclusion and Future Work . . . . . . . . . . . . . . 207

8.1 Summary and Conclusion ................ 207

8.2 Suggestions for Future Work . . . . . . . . . . . . . 217

References ........................ 219

A Parameters of the Models/Controllers . . . . . . . . . . 239

A.1 Parameters of cold start plant model . . . . . . . . . . . 239

A.1.1 Constants ........................ 239

A.1.2 Functions ...................... 239

A.2 Parameters of the Engine SMCs in Chapter $3 \ldots . . . . .241$

A.2.1 Uncertainty-Adaptive SISO SMC . . . . . . . . . . . 241

A.2.2 Uncertainty-Adaptive MIMO SMC . . . . . . . . . . 241

A.3 Parameters of DC Motor Plant Model . . . . . . . . . . . . 241

B PhD Publications . . . . . . . . . . . . . . . . . . 243

B.1 Peer Reviewed Journal Papers . . . . . . . . . . . . . . . . 243

B.1.1 Published Journal Papers ............. 243

B.1.2 Submitted Journal Paper . . . . . . . . . . . . 244

B.2 Peer-Reviewed Conference Papers ............. 244

B.2.1 Submitted Conference Papers ............ 245 
C Program and Data File Summary . . . . . . . . . . . 247

C.1 Chapter 1 . . . . . . . . . . . . . . ...... 247

C.2 Chapter $2 \ldots \ldots \ldots \ldots \ldots \ldots \ldots$. . . . . . . . . . . . . . . .

C.3 Chapter 3 . . . . . . . . . . . . . . . . . . . . . . . . . . 248

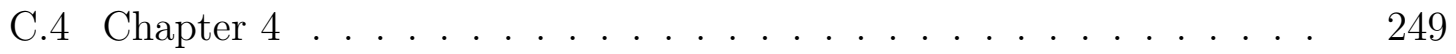

C.5 Chapter $5 \ldots \ldots \ldots \ldots \ldots \ldots$. . . . . . . . . . . . . . .

C.6 Chapter 6 . . . . . . . . . . . . . . . . . . . 251

C.7 Chapter $7 \ldots \ldots \ldots \ldots$

D Letters of Permission .................... 255

D.1 Letter of Permission for Ref [1] (Chapter 2) . . . . . . . 256

D.2 Letter of Permission for Ref [2] (Chapter 2] . . . . . . . 257

D.3 Letter of Permission for Ref [3] (Chapter 3) . . . . . . . . 258

D.4 Letter of Permission for Ref [4] (Chapter 4) . . . . . . 259]

D.5 Letter of Permission for Ref [5] (Chapter 5) . . . . . . . 260

D.6 Letter of Permission for Ref [6] (Chapter 5) . . . . . . . . 261

D.7 Letter of Permission for Ref [9] (Chapter 7) . . . . . . . 262

D.8 Letter of Permission for Figure 2.4 [7] . . . . . . . . . 263 


\section{List of Figures}

1.1 Typical V-cycle for the design of automotive controllers. . . . . . . 2

1.2 Imprecisions on measured signals due to sampling and quantization. 3

1.3 Background of previous literature on controller design against implementation imprecisions. ................. 4

1.4 Overview of model reduction techniques for control systems. . . . . 7

1.5 Organization of this dissertation. . . . . . . . . . . 12

2.1 Computational flow in a typical fixed-point automotive control system.............................. 14

2.2 Proposed early model-based V \& V methodology. . . . . . . . . . . 16

2.3 Process of nonlinear SPA model order reduction. . . . . . . . . 25

2.4 Comparison between measured and simulated cumulative tailpipe HC emission. ........................ 30

2.5 Hankel singular values for cold start emission model. . . . . . . . . 32

2.6 Comparison of tailpipe $\mathrm{HC}$ flow rates predictions by full state and reduced models. .................... 33

2.7 Structure of the designed cold start controller. . . . . . . . . . . . . 35 
2.8 Comparison of tracking performance of the designed SMCs. . . . . . 38

2.9 Comparison of cold start emission control the full order SMC and the nonlinear reduced-order SPA SMC. . . . . . . . . . . . 40

2.10 Effect of data types, quantization levels and sampling rates on the performance of the cold start controller. . . . . . . . . . . 43

3.1 Proposed adaptive SISO SMC to account for implementation imprecisions............................ 51

3.2 Effects of ADC imprecisions on baseline SMC for tracking AFR, $T_{e x h}$, and $\omega_{e} \ldots \ldots \ldots \ldots \ldots \ldots$

3.3 Verification results of the uncertainty gains from the SISO adaptive SMC ......................... 57

3.4 Impact of AFR and engine speed control on each other from SISO

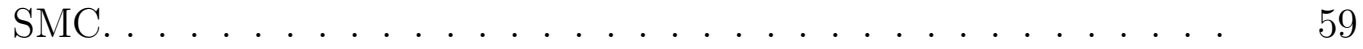

3.5 Schematic of the adaptive MIMO SMC under implementation imprecisions. . . . . . . . . . . . . . . . . . 62

3.6 Schematic of the processor-in-the-loop (PIL) setup for real-time controller verification. . . . . . . . . . . . . . 63

3.7 Real-time verification of the designed adaptive MIMO controller for tracking AFR, exhaust temperature, and engine speed under ADC imprecisions. . . . . . . . . . . . . . . . 66 
3.8 Real-time results of tracking the desired trajectories from ideal, baseline, and adaptive MIMO SMCs to control HC emission. . . . . . . 67

3.9 Cumulative tailpipe HC emission from ideal, baseline, and proposed adaptive MIMO SMCs. . . . . . . . . . . . . . . . 69

4.1 Uncertainty on a measured signal due to sampling. . . . . . . . . 74

4.2 Schematic of the DSMC with online estimations of uncertainties on measured and control signals. . . . . . . . . . . . . . 779

4.3 Schematic of the modeled DC motor. . . . . . . . . . . . 82

4.4 Comparison of actual and predicted uncertainties due to sampling and quantization. ........................... 83

4.5 DC motor speed control using the baseline SMC and the modified DSMC.......................... 85

4.6 Results of engine control under ADC imprecisions. . . . . . . . 88

4.7 Results of DSMC real-time verification for the DC motor speed control. ............................. 91

4.8 Real-time verification for the engine control using the proposed DMSC .............................. 92

5.1 Schematic of the adaptive DSMC with online estimations of uncertainties on measured and control signals. . . . . . . . . . . . . 101

5.2 Block diagram of the engine cold start control system. . . . . . . . . 102 
5.3 Comparison of actual and predicted uncertainties due to sampling and quantization on measured variables of the SI engine. . . . . . . . 108

5.4 The impacts of unknown uncertainty terms on the engine dynamics inside the DSMC and how the adaptation mechanisms drive the model with error to its nominal value. . . . . . . . . . . . . . . . 109

5.5 Convergence results of unknown engine model multiplicative and additive terms. . . . . . . . . . . . . . . . . 110

5.6 Comparison between baseline adaptive and non-adaptive engine DSMCs under ADC imprecisions and model uncertainties. . . . . . 111

5.7 Results of engine control under ADC imprecisions and model uncertainties. . . . . . . . . . . . . . . 113

5.8 Effect of sampling time and quantization level on the tracking performance of the engine controller with and without the predicted ADC uncertainties. . . . . . . . . . . . . . . . 114

5.9 Effects of incorporating the predicted ADC uncertainties and the adaptation laws on the DSMC robustness characteristics. . . . . . . 116

5.10 Convergence results of the unknown additive and multiplicative terms in the DC motor model. . . . . . . . . . . . . . . . . . . 127

5.11 DC motor speed control using the baseline adaptive DSMC and the modified adaptive DSMC with predicted ADC uncertainties. . . . . 127 
5.12 Real-time verification result of adaptive robust DSMC for the DC motor speed control. . . . . . . . . . . . . . . . . . 128

5.13 Real-time verification results of the adaptive robust DSMC for the engine control case study. . . . . . . . . . . . . . . . . . . . 129

6.1 Engine tracking results by the first and second order SISO DSMCs. 161

6.2 Results of desired trajectories tracking from SISO Baseline $1^{\text {st }}$ and $2^{\text {nd }}$ order DSMCs, and MIMO $2^{\text {nd }}$ order DSMC with predicted ADC uncertainties. ........................ 162

6.3 Disturbing load torque profile. . . . . . . . . . . . . 165

6.4 Disturbance rejection performance of the SISO baseline first and second order DSMCs. . . . . . . . . . . . . . . . . . . . . . . 166

6.5 Disturbance rejection performance of the SISO and MIMO baseline first and second order DSMCs. . . . . . . . . . . . . . . . 167

6.6 Results of unknown multiplicative parameters convergences. . . . . 170

6.7 Results of engine control under model uncertainties. . . . . . . . . . 170

6.8 Comparison among the speed tracking results of the first and second order DSMCs for different sampling rates. . . . . . . . . . . 174

6.9 Comparison between the first and second order DSMCs in tracking the desired speed trajectory of the DC motor for different quantization levels. ........................ 175 
6.10 Comparison between the MIMO and SISO second order DSMCs, with and without predicted ADC uncertainties, in tracking the desired speed trajectory under extreme ADC uncertainties. . . . . . . . . 176

6.11 Disturbance rejection performance of the SISO and MIMO second order DSMCs. . . . . . . . . . . . . . . . 177

6.12 Disturbance rejection performance of the SISO and MIMO second order DSMCs, with and without predicted ADC uncertainties. . . . . 178

6.13 Real-time verification result of the DC motor speed control using the adaptive first order SISO DSMC and the modified adaptive second order MIMO DSMC with predicted ADC uncertainties. . . . . . . . 179

7.1 Throttle experimental setup. . . . . . . . . . . . . . . . . . . 184

7.2 Schematic of the electronic throttle actuator. . . . . . . . . 186

7.3 Effect of nonlinear return spring with variable stiffness 12 on throttle torque-position response. . . . . . . . . . . . . . 186

7.4 Open-loop experimental results of testing the throttle. . . . . . . 188

7.5 Effects of nonlinear return spring on the throttle position dynamic at different input frequencies. . . . . . . . . . . . . . . . 188

7.6 Results of throttle model verification against two sets of experimental data. ........................... 192

7.7 Simulation results of the baseline DSMC for tracking the desired throttle position. . . . . . . . . . . . . . . 196 
7.8 Comparison of actual and predicted uncertainties due to sampling and quantization on measured throttle position. . . . . . . . . . 197

7.9 Simulation results of the baseline DSMC and robust DSMC with predicted sampling uncertainties for tracking the desired throttle position. ............................ 198

7.10 Simulation results of the baseline DSMC and robust DSMC with predicted ADC uncertainties for tracking the desired throttle position.

7.11 Experimental testing results of the baseline DSMC for tracking the desired throttle position . . . . . . . . . . . . . 200

7.12 Experimental testing results of the baseline DSMC for tracking the desired throttle position. . . . . . . . . . . . . .

7.13 Experimental testing results of the baseline and robust DSMC for tracking the desired throttle position. . . . . . . . . . . 201

7.14 Experimental testing results of the baseline and robust DSMC for tracking the desired throttle position. . . . . . . . . . .

7.15 Simulation results of comparing the baseline second order DSMC with the baseline first order DSMC for tracking the desired throttle posi-

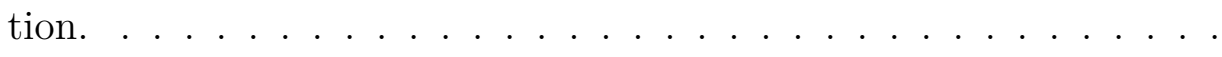

D.1 Letter of Permission for Ref [1]. . . . . . . . . 256

D.2 Letter of Permission for Ref [2] . . . . . . . . . . 257

D.3 Letter of Permission for Ref [3]. . . . . . . . . . . 258 
D.4 Letter of Permission for Ref 4 . . . . . . . . . . . . 259

D.5 Letter of Permission for Ref $[5] \ldots \ldots \ldots \ldots \ldots$

D.6 Letter of Permission for Ref $[6] \ldots \ldots \ldots$

D.7 Letter of Permission for Ref $[9] \ldots \ldots \ldots \ldots$

D.8 Letter of Permission for Figure $2.4[7] . \ldots \ldots \ldots \ldots$ 


\section{List of Tables}

2.1 Baseline Condition Used in the Robustness Analysis. . . . . . . . . 41

3.1 Mean value of the tracking errors from the baseline and adaptive SISO SMCs. ......................... 57

3.2 Comparison between the baseline and MIMO adaptive SMCs in terms of mean error in tracking the desired engine trajectories. . . . . . 65

4.1 Mean and Standard Deviation of ADC Uncertainty Prediction Errors for the DC Motor Model. . . . . . . . . . . . . . . . .

4.2 Mean and Standard Deviation errors for Predicting the ADC Uncertainty for the Engine Model's Measured Signals. . . . . . . . . . .

4.3 Mean and Standard Deviation of Tracking Errors. Values Inside the Parentheses Show the Resulting Improvement Compared to The Baseline SMC. . . . . . . . . . . . . . . . .

5.1 Mean and standard deviation of ADC uncertainty prediction errors for the SI engine model. . . . . . . . . . . . . . . . . 
5.2 Mean value of the errors in trajectory tracking, and estimating the engine dynamics from the non-adaptive and adaptive DSMCs. . . . 112

6.1 Mean of Tracking Errors. . . . . . . . . . . . . . . . . 161

7.1 Identified parameters of the throttle model. . . . . . . . . . 192

7.2 Comparison results between the first and second order DSMCs for tracking the desired throttle position. . . . . . . . . . . . . 203

A.1 Plant model constants. . . . . . . . . . . . . . . . 239

A.2 Gains $(\lambda, \gamma)$ and boundary layers $(\phi)$ of designed SISO adaptive SMC for the engine control. . . . . . . . . . . . . . 241

A.3 Gains and boundary layers of designed MIMO adaptive SMC for the engine control. ..................... 241

A.4 DC motor model constants. . . . . . . . . . . . . . . . 241

C.1 Chapter 1 Figure files. . . . . . . . . . . . . . . . . 247

C.2 Chapter 2 Figure files. . . . . . . . . . . . . . 247

C.3 Chapter 2 MATLAB script and SIMULINK files. . . . . . . . 248

C.4 Chapter 3 Figure files. . . . . . . . . . . . . . . . 248

C.5 Chapter 3 MATLAB script and SIMULINK files. . . . . . . . . 249

C.6 Chapter 4 Figure files. . . . . . . . . . . . . . . 249

C.7 Chapter 4 MATLAB script and SIMULINK files (Engine case study). 249 
C.8 Chapter 4 MATLAB script and SIMULINK files (DC motor case study).......................... 250

C.9 Chapter 5 Figure files. . . . . . . . . . . . . . . . . . . 250

C.10 Chapter 5 MATLAB script and SIMULINK files (Engine case study). $\quad 250$

C.11 Chapter 5 MATLAB script and SIMULINK files (DC motor case study)............................ 251

C.12 Chapter 6 Figure files. . . . . . . . . . . . . . . . . . . . . 251

C.13 Chapter 6 MATLAB script and SIMULINK files (Engine case study). 251

C.14 Chapter 6 MATLAB script and SIMULINK files (DC motor case study)............................... 252

C.15 Chapter 7 Figure files. . . . . . . . . . . . . . . . . . . . 252

C.16 Chapter 7 MATLAB script, SIMULINK files, and experimental data. 253 



\section{Preface}

The manuscript and the results of this dissertation are mainly based on five journal papers and seven conference papers. Publishers of these papers have granted the permissions found in Appendix D. The contribution of the author of this dissertation as well as the contributions of the co-authors for each of the papers are as follows:

- Contribution for Chapter 2 ([1, 2]): Developing the nonlinear model order reduction techniques based on singular perturbation approximation, design of the reduced order SMC, evaluation of the designed controllers, and writing these sections have been done by the author of this dissertation, M. R. Amini. Dr. M. Shahbakhti's contributions included development of the early model-based SIL verification methodology, developing a method of characterizing and evaluating robustness of the controllers against ADC imprecisions, design of PIL setting for real-time controller testing, controller structure design, analysis of the modeling and control results, and manuscript editing. Nonlinear model linearizion and computing the balanced realization form of the engine model were done by Mr. S. Asami. Mr. J. Li has evaluated the controller performance under implementation imprecisions. Dr. J. K. Hedrick and Dr. A. Ghaffari provided technical comments during the course of writing these papers.

- Contribution for Chapter 3 ([3]): Developing the concept of uncertaintyadaptive SMC, design of the SISO/MIMO SMC for engine cold start case study, 
design of the processor-in-the-loop (PIL) setup, conducting the real-time controller tests, and writing this paper have been done by the author of this dissertation, M. R. Amini. Dr. M. Shahbakhti guided the research during the course of the work, leading to this publication. He also contributed in analysis of the results and manuscript editing. Dr. J. K. Hedrick provided technical comments during the course of this paper.

- Contribution for Chapter 4 ([4]): Developing the sampling and quantization uncertainty prediction and propagation technique, design of the DSMC with incorporated ADC imprecisions, evaluation of the designed controller for combustion engine and DC motor case studies under implementation imprecisions, real-time testing of the controllers, and writing this paper have been done by the author of this dissertation, M. R. Amini. Dr. M. Shahbakhti guided the research during the course of the work, leading to this publication. He also contributed in analysis of the results and manuscript editing. Dr. J. K. Hedrick provided technical comments during the course of this paper.

- Contribution for Chapter $5([5,6])$ : Developing the adaptive DSMC with incorporated ADC imprecisions, stability analysis of the designed controller based on Lyapunov stability theorem, deriving the adaptation laws for handling additive type of modeling uncertainty, evaluation of the designed controller for combustion engine and DC motor case studies under modeling and implementation imprecisions, real-time testing of the controllers, and writing these papers have 
been done by the author of this dissertation, M. R. Amini. Dr. M. Shahbakhti guided the research during the course of the work, leading to these publications. He also contributed in analysis of the results and manuscript editing. Dr. S. Pan has developed the adaptive DSMC formulation for handling multiplicative type of modeling uncertainty, and provided technical comments on extending the adaptation algorithm to additive type of modeling uncertainty and inclusion of implementation imprecisions into the adaptive DSMC formulation, stability analysis of the proposed controller, and manuscript editing. Dr. J. K. Hedrick provided technical comments during the course of these papers.

- Contribution for Chapter 6] ([8]): Developing the concept of second order SISO/MIMO adaptive DSMC, inclusion of the predicted implementation imprecisions in the second order DSMC, Lyapunov-based stability analysis, deriving the adaptation laws for handling multiplicative type of modeling uncertainty, evaluation of the designed controller for combustion engine and DC motor case studies under modeling uncertainty, implementation imprecisions, and external disturbances, real-time testing of the controllers, and writing this paper have been done by the author of this dissertation, M. R. Amini. Dr. M. Shahbakhti guided the research during the course of the work, leading to this publication. He also contributed in analysis of the results and manuscript editing. Dr. S. Pan has provided technical comments on the stability analysis of the proposed second order controller, and manuscript editing. Dr. J. K. Hedrick provided technical comments during the course of writing this paper. 
- Contribution for Chapter 7 ([9]): Data collection, analysis of the experimental data, modeling, validation of the throttle model, design and experimental testing of the model-based throttle DSMC, and writing this paper have been done by the author of this dissertation M. R. Amini. Dr. M. Razmara helped with collecting the experimental data. Dr. M. Shahbakhti guided the research during the course of the work, leading to this publication. He also contributed in analysis of the results and manuscript editing. 


\section{Acknowledgments}

It is a great pleasure for me to take this opportunity to express my gratitude to my PhD advisor, Dr. Mahdi Shahbakhti, for all his encouragement, guidance, and support during the past seven years. I had the opportunity to work under his supervision during my MS and $\mathrm{PhD}$, and develop my research background in the field of automotive powertrain controls. Dr. Shahbakhti's enthusiasm for research has been a great motivation for me to conduct research, publish several journal and conference articles, and pursue my future career in academia. Among all the lessons I have learned from him, I will take the professionalism and ethics in the engineering profession, as the life long lessons.

I gratefully thank Prof. Rush D. Robinett III, who not only served on my dissertation committee, but he has been also a wonderful counselor and mentor for me. He has always given me novel ideas for my research, and valuable advice for my future academic life.

It has been a great honor for me to have Prof. J. Karl Hedrick (1944-2017) as my mentor and PhD committee member since 2014. He has been a role model for me as a great researcher, and an excellent professor, in the field of nonlinear control systems. I am pleased that I had the opportunity to deliver a workshop at 2016 American 
Control conference (ACC) in Boston in collaboration with him.

I graciously acknowledge Dr. Selina Pan from Research and Innovation Center of Ford Motor Company for assisting me in developing adaptive controls theory, her support, and providing me with solid comments and discussions through email communications, and online meetings.

I am grateful to Dr. Ken Butts from Toyota Technical Center for his technical comments and support during the course of my PhD. Also I would like to thank United States National Science Foundation (NSF), and Dr. Predebon and Dr. Friedrich from MTU Mechanical Engineering department for facilitating my PhD study by providing financial support.

My special thanks goes to Dr. Darrell L. Robinette, Dr. Jie Sun, and Dr. Wayne W. Weaver who agreed to serve on my dissertation committee, and provided me with valuable inputs to improve my dissertation quality.

I would like to thank several individual colleagues at MTU, who have provided both encouragement and technical help during my PhD: Dr. Meysam Razmara, as one of my best friends, Dr. Amir Rezaei and Barzin Moridian for helping me to build up the required skills for configuring the processor-in-the-loop setup, Dr. Ehsan Taheri for his encouragement and support, Dr. Nancy Barr for her editorial comments, and Jayant Arora and Kaushik Kannan for their help in collecting the experimental 
throttle data. Additionally, I would like to thank Andreas Hansen and Eric Choi from University of California, Berkeley for their technical comments. I would like to acknowledge my dear friends all around the globe: Arash Jamali, Amirhossein Hosseini, Kamran Amini, Mohammad Reza Ramezani, Mohammad Abbasi, Kianoosh Azizi, Mohammad Naderi, Mohammad Salemi, Kian Mirniazy, Ehsan Omidi, Mehdi Mortazavi, and Bjorn Eberth.

Lastly, I owe deep gratitude to my family for all the spiritual support, love, and encouragement: my lovely wife, Sanaz, my dear mother, Molouk, my sacrificing father, Jamal, my little sister, Sarah, my brother, Alireza, my mother-in-law, Shahnaz, and my father-in-law, Reza. 



\section{List of Abbreviations}

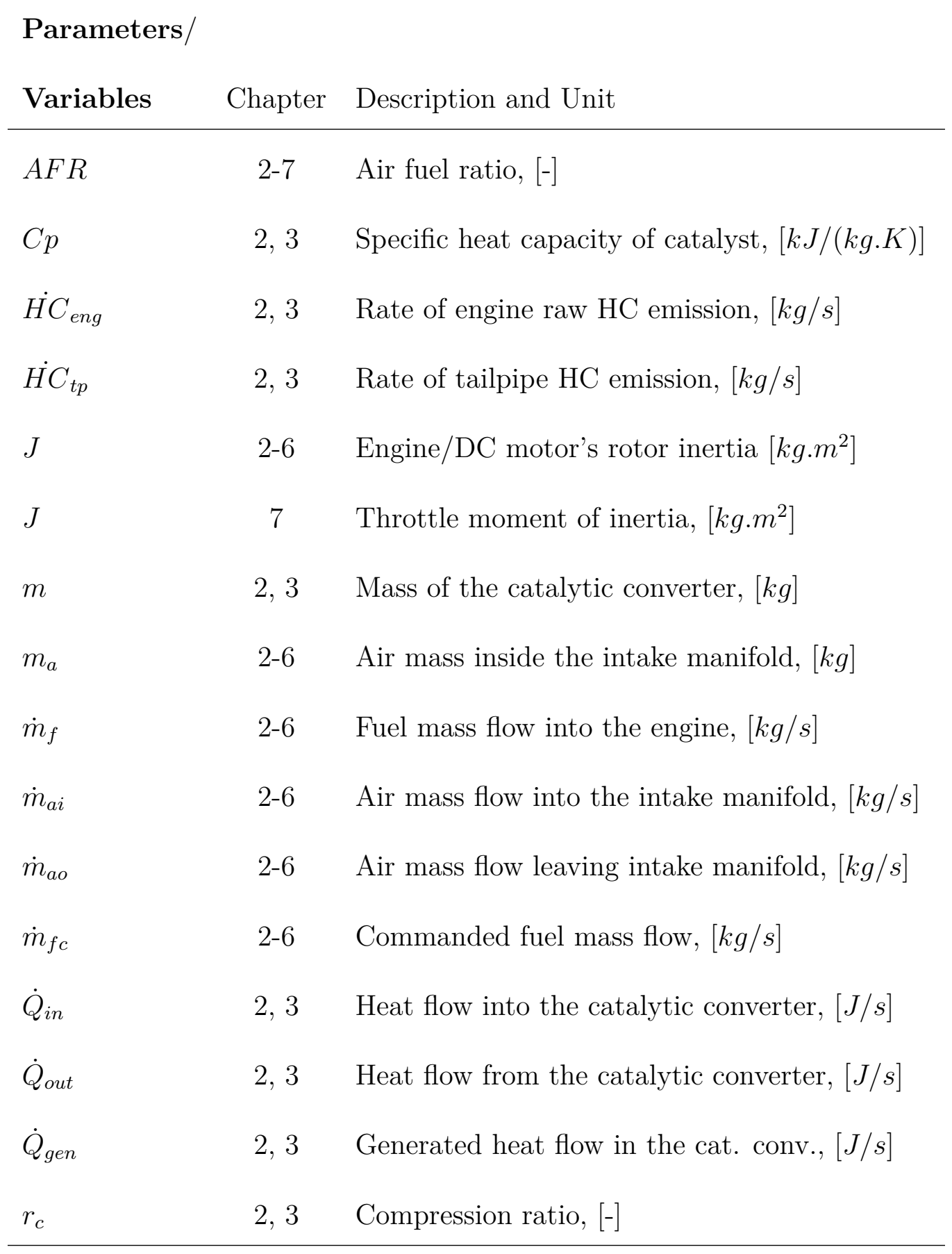




\begin{tabular}{|c|c|c|}
\hline$T_{\text {cat }}$ & 2.3 & Catalytic converter temperature, $[K]$ \\
\hline$T_{e x h}$ & $2+6$ & Exhaust gas temperature, $[K]$ \\
\hline$\Delta$ & $2+6$ & Spark timing, $\left[{ }^{\circ}\right.$ ATDC $]$ \\
\hline$\eta_{\text {cat }}$ & 2,3 & Catalytic converter conversion efficiency, [-] \\
\hline$\eta_{v o l}$ & & Engine volumetric efficiency, [-] \\
\hline$\lambda$ & & Gradient of sliding surface, $[-]$ \\
\hline$\omega_{e}$ & 2 & Engine speed, $[\mathrm{rad} / \mathrm{s}]$ \\
\hline$N$ & 4 & Engine speed, $[R P M]$ \\
\hline$N$ & 1 & Total gear ration from DC motor shaft to throttle shaft, $[-]$ \\
\hline$\tau_{f}$ & $24 \mid$ & Fuel time constant, $[1 / s]$ \\
\hline$\tau_{f}$ & 7 & Dry friction torque, $[N . m]$ \\
\hline$\tau_{e}$ & 246 & Exhaust gas time constant, $[1 / s]$ \\
\hline$H S V$ & 2 & Hankel singular values , [-] \\
\hline$T_{s}$ & 2,3 & Sampling Time, $[\mathrm{sec}]$ \\
\hline$T$ & 4.7 & Sampling time, $[s]$ \\
\hline$n$ & $3-7$ & Quantization Level, $[$ bit $]$ \\
\hline$R$ & 4.5 & Electrical resistance, $[\Omega]$ \\
\hline$L$ & 4.5 & Electrical inductance, $[\mathrm{H}]$ \\
\hline$\Gamma$ & 4.5 & Generated torque on DC motor's rotor, $[$ N.m] \\
\hline$k_{f}$ & \begin{tabular}{|l|l|}
4 & 5
\end{tabular} & Mechanical dimpling, [N.m.s] \\
\hline$k_{m}$ & $4 \mid 5$ & Motor torque constant, $[\mathrm{N} . \mathrm{m} / \mathrm{A}]$ \\
\hline
\end{tabular}




\begin{tabular}{|c|c|c|}
\hline$k_{b}$ & 4.5 & Electromotive force constant, [V.s/rad] \\
\hline$\rho$ & 4.7 & Tunable first order DSMC gain, [-] \\
\hline $\mathcal{P}$ & 5 & Tunable first order DSMC Matrix, [-] \\
\hline$\alpha$ & $5 \mid$ & Additive uncertainty term, $[-]$ \\
\hline$\alpha$ & 6 & Multiplicative uncertainty term, $[-]$ \\
\hline$\beta$ & 5 & Multiplicative uncertainty term, $[-]$ \\
\hline$\beta$ & 6 & Tunable second order DSMC gain, [-] \\
\hline$\beta$ & 6 & Tunable second order DSMC Matrix, [-] \\
\hline$\rho_{\beta}$ & $|5|$ & Adaptation gain for multiplicative uncertainty, $[-]$ \\
\hline$\rho_{\alpha}$ & 5 & Adaptation gain for additive uncertainty, $[-]$ \\
\hline$\rho_{\alpha}$ & $6 \mid$ & Multiplicative adaptation gain, [-] \\
\hline$\theta$ & $4-6$ & DC motor rotational speed, $[\mathrm{rad} / \mathrm{s}]$ \\
\hline $\mathcal{I}$ & 4.7 & DC motor armature current, $[A]$ \\
\hline$\xi$ & 6 & Second order sliding mode variable, $[-]$ \\
\hline$\xi$ & 7 & Vertical force, $[N]$ \\
\hline $\mathcal{N}$ & 6 & Sliding mode order, [-] \\
\hline$\varphi_{M}$ & 7 & DC motor shaft position, $[\mathrm{rad}]$ \\
\hline$\varphi$ & 7 & Throttle position, $[\mathrm{rad}]$ \\
\hline$k_{e m f}$ & 7 & Back electromotive force constant, $[\mathrm{V} . s / \mathrm{rad}]$ \\
\hline$\eta$ & 7 & Efficiency of the mechanical system, $[-]$ \\
\hline$\tau_{M}$ & $7 \mid$ & Electrical torque on DC motor shaft, $[N . m]$ \\
\hline
\end{tabular}




\begin{tabular}{|c|c|c|}
\hline$\tau$ & 7 & Electrical torque on throttle shaft, $[N . m]$ \\
\hline$\tau_{v}$ & 7 & Mechanical viscous torque, $[N . m]$ \\
\hline$\tau_{s}$ & 7 & Nonlinear spring torque, $[N . m]$ \\
\hline$b_{m e c h}$ & 7 & Mechanical viscous damping constant, $[$ N.m.s/rad $]$ \\
\hline$k_{s}$ & 7 & Stiffness of the return spring, $[N . m / r a d]$ \\
\hline$\mu$ & 7 & Dry friction constant, $[m]$ \\
\hline
\end{tabular}




\begin{tabular}{ll} 
Acronyms & \\
ADC & Analog-to-digital converter \\
AFR & Air-fuel ratio \\
APC & Adaptive pulse control \\
ATDC & After top dead center \\
CSV & Control software verification \\
DC & Direct current \\
DSMC & Discrete sliding mode control \\
ECU & Electronic control unit \\
FSR & Full scale range \\
HC & Hydrocarbon \\
HIL & Hardware-in-the-loop \\
ICE & Internal combustion engine \\
I/O & Input/output \\
MIL & Model-in-the-loop \\
MIMO & Multi-input Multi-output \\
LPV & Linear parameter varying \\
PID & Proportional-integral-derivative \\
PIL & Processor-in-the-loop \\
RCP & Rapid control prototype \\
\hline
\end{tabular}




\section{Acronyms}

SIL Software-in-the-loop

SISO Single-input single-output

SMC Sliding mode control

SPA Singular perturbation approximation

TPS Throttle position sensor

V\&V Verification and validation 



\section{Abstract}

Bridging the gap between designed and implemented model-based controllers is a major challenge in the design cycle of industrial controllers. This gap is mainly created due to (i) digital implementation of controller software that introduces sampling and quantization imprecisions via analog-to-digital conversion (ADC), and (ii) uncertainties in the modeled plant's dynamics, which directly propagate through the controller structure. The failure to identify and handle these implementation and model uncertainties results in undesirable controller performance and costly iterative loops for completing the controller verification and validation $(\mathrm{V} \& \mathrm{~V})$ process.

This PhD dissertation develops a novel theoretical framework to design controllers that are robust to implementation imprecision and uncertainties within the models. The proposed control framework is generic and applicable to a wide range of nonlinear control systems. The final outcome from this study is an uncertainty/imprecisionsadaptive, easily verifiable, and robust control theory framework that minimizes $\mathrm{V} \& \mathrm{~V}$ iterations in the design of complex nonlinear control systems.

The concept of sliding mode controls (SMC) is used in this study as the baseline to construct an easily verifiable model-based controller design framework. SMC is a robust and computationally efficient controller design technique for highly nonlinear systems, in the presence of model and external uncertainties. The SMC structure 
allows for further modification to improve the controller robustness against implementation imprecisions, and compensate for the uncertainties within the plant model.

First, the conventional continuous-time SMC design is improved by: (i) developing a reduced-order controller based on a novel model order reduction technique. The reduced order SMC shows better performance, since it uses a balanced realization form of the plant model and reduces the destructive internal interaction among different states of the system. (ii) developing an uncertainty-adaptive SMC with improved robustness against implementation imprecisions. Second, the continuous-time SMC design is converted to a discrete-time SMC (DSMC). The baseline first order DSMC structure is improved by: (i) inclusion of the ADC imprecisions knowledge via a generic sampling and quantization uncertainty prediction mechanism which enables higher robustness against implementation imprecisions, (ii) deriving the adaptation laws via a Lyapunov stability analysis to overcome uncertainties within the plant model, and (iii) developing a second order adaptive DSMC with predicted ADC imprecisions, which provides faster and more robust performance under modeling and implementation imprecisions, in comparison with the first order DSMC.

The developed control theories from this $\mathrm{PhD}$ dissertation have been evaluated in real-time for two automotive powertrain case studies, including highly nonlinear combustion engine, and linear DC motor control problems. Moreover, the DSMC with predicted ADC imprecisions is experimentally tested and verified on an electronic air throttle body testbed for model-based position tracking purpose. 


\section{Chapter 1}

\section{Introduction}

Verification and Validation $(\mathrm{V} \& \mathrm{~V})$ in a controller design cycle ensures that (i) the implemented controller in an electronic control unit (ECU) accurately represents the conceptual description and specifications of the designed controller; (ii) the designed controller fulfills intended control targets. V\&V for complex dynamic systems is too costly and time consuming. For instance, the current ECUs in a Lexus LS460 vehicle has a program size of seven million lines [10]. The traditional V\&V cycle of various ECU functions, shown in Figure 1.1, is completed with multiple software-in-the-loop (SIL), processor-in-the-loop (PIL), and hardware-in-the-loop (HIL) iterations which are complex and time consuming. Using the current technology, the $\mathrm{V} \& \mathrm{~V}$ process for typical automotive ECUs can take about two years, and it can easily cost over several million dollars. The cost and development time of the ECU dramatically 
increase when the complexity of an automotive system increases (e.g., a hybrid electric vehicle). Reducing cost and time of the $\mathrm{V} \& \mathrm{~V}$ is a major challenge for all complex control systems and this dissertation aims to develop and illustrate a model-based controller design methodology for reducing V\&V efforts.

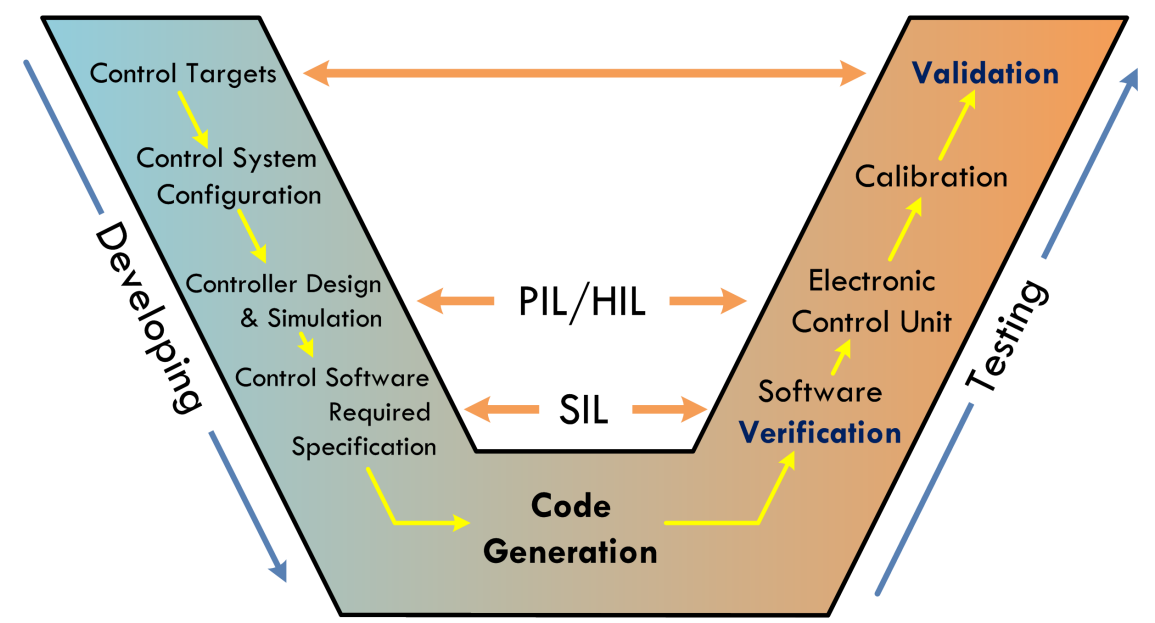

Figure 1.1: Typical V-cycle for the design of automotive controllers.

There are two main sources of uncertainties in the design cycle of industrial modelbased controllers that cause errors in a V\&V cycle. First, the imprecisions arise during digital implementation of the controller upon analog-to-digital (ADC) conversion of signals at the input/output (I/O) of the controller due to sampling and quantization. Second, uncertainties exist in the modeled plant's dynamics used in the controller formulation. If these uncertainties could be identified and fixed during the early stages of the controller software design, the $\mathrm{V} \& \mathrm{~V}$ process would cost 10 times less [11, 12]. Thus, designing a robust and adaptive controller that tries to minimize the impact of implementation and model imprecisions can reduce the V\&V cost significantly. 


\section{$1.1 \quad$ Background}

A digital control unit acquires the feedback data from the plant through analog to digital converter (ADC) which samples and quantizes the measured signals. Figure 1.2 shows the analog versus sampled and quantized signal after ADC. As seen in Figure 1.2, significant imprecisions can be caused by ADC, depending on sampling time and quantization level. Data acquisition systems quantize the sampled data from measurements to generate digital signals with a quantization level that is supported by the ECU. Quantization is not usually considered in the controller design and introduces round-off error when controller is implemented. As discussed, sampling and quantization imprecisions have major effects on the controller performance. If these imprecisions are not considered during the early stages of the controller design, they could lead to failure in the controller performance and consequently time-consuming $\mathrm{V} \& \mathrm{~V}$ iterations [12].

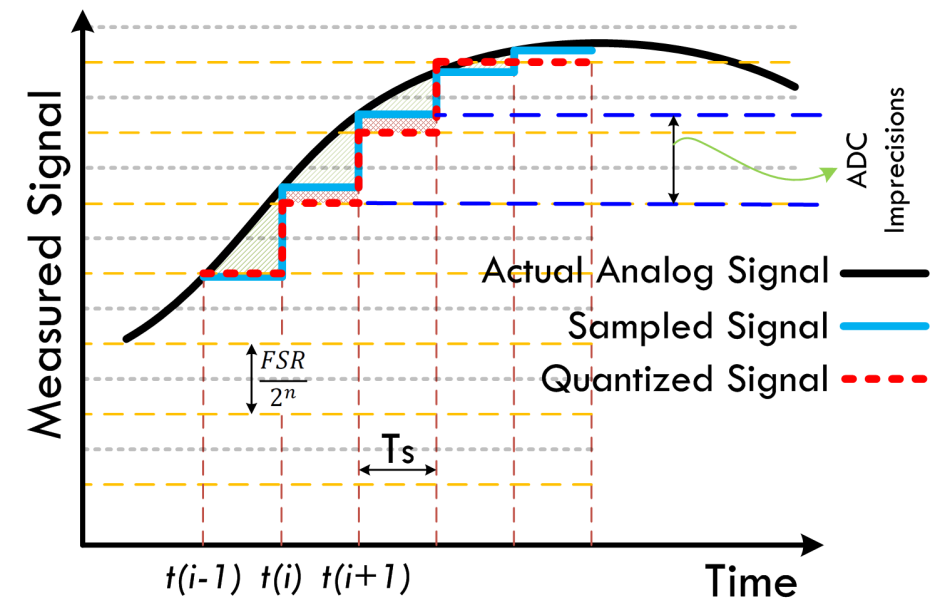

Figure 1.2: Imprecisions on measured signals due to sampling and quantization ( $T_{S}$ is sampling time, FSR is full scale range of the measured signal, and $n$ is number of ADC bits and shows the ADC quantization level). 
The major previous studies, that focused on analyzing and developing controller designs to overcome implementation imprecisions, are shown in Figure 1.3. Control design techniques with sampled data have been studied extensively, while few works have addressed data quantization imprecisions. State feedback function selection for favorable stability characteristics is a major challenge in the controller design process [13]. Other investigated challenges include controller design to guarantee global asymptotic stability at the origin under discrete-time implementation and finding the permissible bounds of sampling period to achieve asymptotic stability [13, 14]. Verifying a controller's stability for a given bounded set against quantization imprecisions is the other problem which has been studied in [15] and [16].

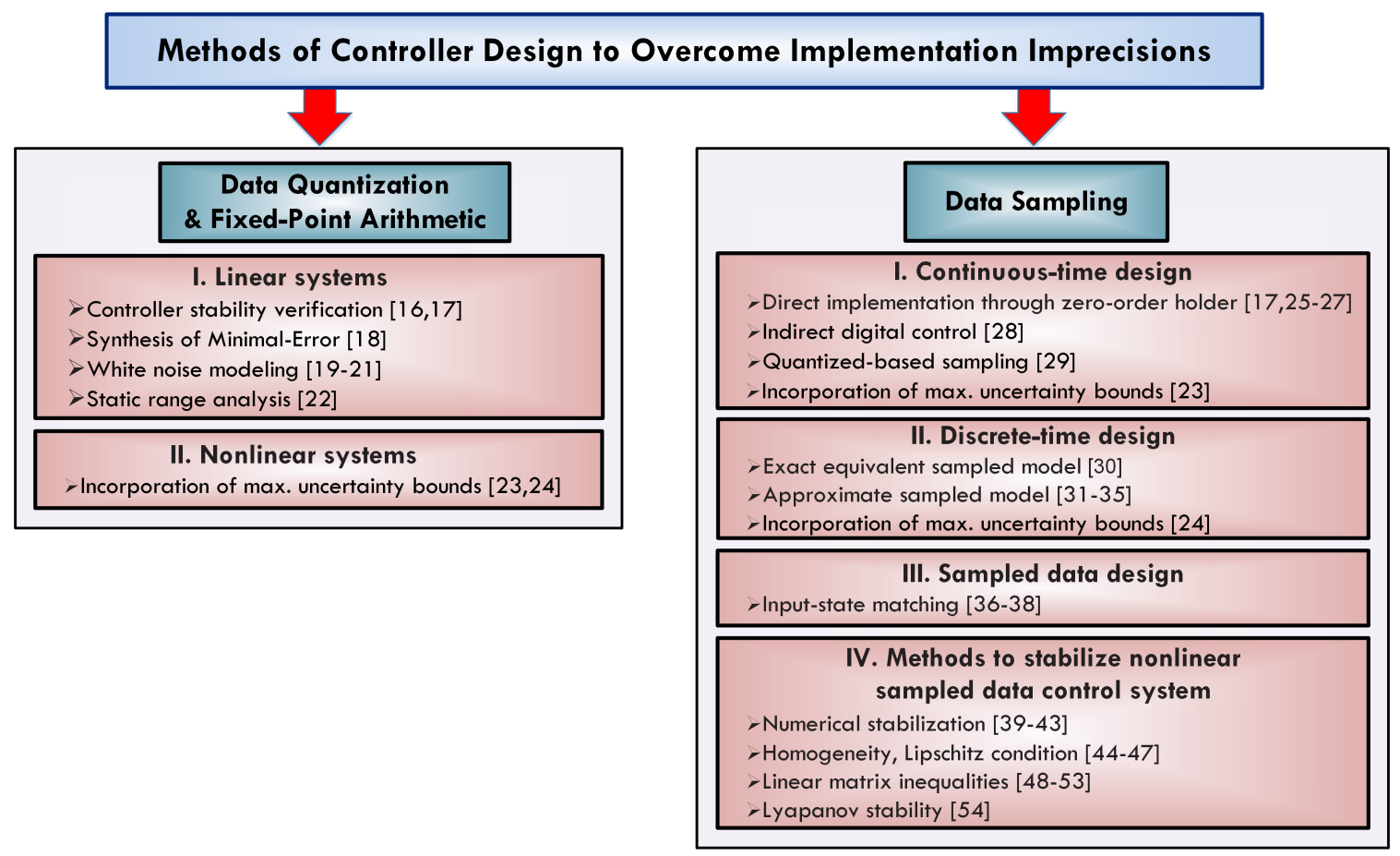

Figure 1.3: Background of previous literature on controller design against implementation imprecisions [16, 17, 18, 19, 20, 21, 22, 23, 24, 25, 26, 27, $28,29,30,31,32,33,34,35,36,37,38,39,40,41,42,43,44,45,46,47,48$, $49,50,51,52,53,54$. 
Sliding Mode Controls: A sliding mode controller (SMC) is a robust controller that can be applied to both linear and nonlinear systems, and allows for dealing with uncertainties, un-modeled dynamics, and disturbances. SMC design converts a complicated dynamic system into a first-order system through a sliding surface transformation. Previous studies in the literature have demonstrated the capabilities of SMC in handling implementation and model uncertainties. There are two main groups in which the SMC design with robustness to implementation imprecisions can be studied: (i) continuous-time designs, and (ii) discrete-time designs. For the first group, results in [23] showed that the robustness of the implemented controller against ADC uncertainties can be improved by incorporating the maximum ADC uncertainty bounds into the controller equations. Converting the controller design from a single-input singleoutput (SISO) to a multi-input multi-output (MIMO) formulation is another effective approach to enhance controller robustness by minimizing the correlation between the sliding variables via a nonlinear balanced realization method [55].

Handling the modeling uncertainty in SMC design has been the subject of few previous works in the literature. The model uncertainty/mismatch has been addressed using non-adaptive [56], continuous-time adaptive [57], discrete-time adaptive [58, 59], and neural network-based [60, 61] SMC designs. The adaptive DSMC formulation from [62] presents a generic solution for removing a multiplicative type of model uncertainty for a general class of nonlinear systems. Development of a generic adaptive 
first and second order SISO/MIMO DSMCs with incorporated sampling and quantization imprecisions, and adaptation against two types of modeling uncertainties (multiplicative and additive), is the main solution which is proposed in this dissertation to construct an easily verifiable model-based controller design framework.

Model Order Reduction: One important requirement for developing a model-based control strategy is having an accurate and computationally efficient low-order physical model for real-time control. This model should have the capability to be easily implemented and utilized in the design of model-based controllers to evaluate system response under real environment conditions and uncertainties. One approach for simplifying the controller structure, reducing the calculation load, and reducing the controller calibration effort is by utilizing methods of model order reduction shown in Figure 1.4. Three groups are identified in Figure 1.4. The first group includes parameter space reduction methods. These methods reduce controller calibration load through identifying and removing insensitive parameters. The second group is greybox modeling approach which combines physical (clear-box) models and empirical (black-box) models and tries to develop high fidelity models [63]. The third group includes plant order/state reduction methods. These methods attempt to evaluate system's dynamics and eliminate fast and low energetic states of system's dynamics. In order to keep the nonlinearity of the plant, nonlinear state/order reduction methods should be applied on the engine model. Plant state/order reductions methods for linear dynamical systems are described in detail in [64, 65, 66]. One well known 
linear reduction method is the singular perturbation approximation (SPA) method. This method divides system dynamics into fast and slow parts [65] and focuses on the slow part as the important part of the system's dynamics. SPA will be discussed later in detail in Chapter 2.

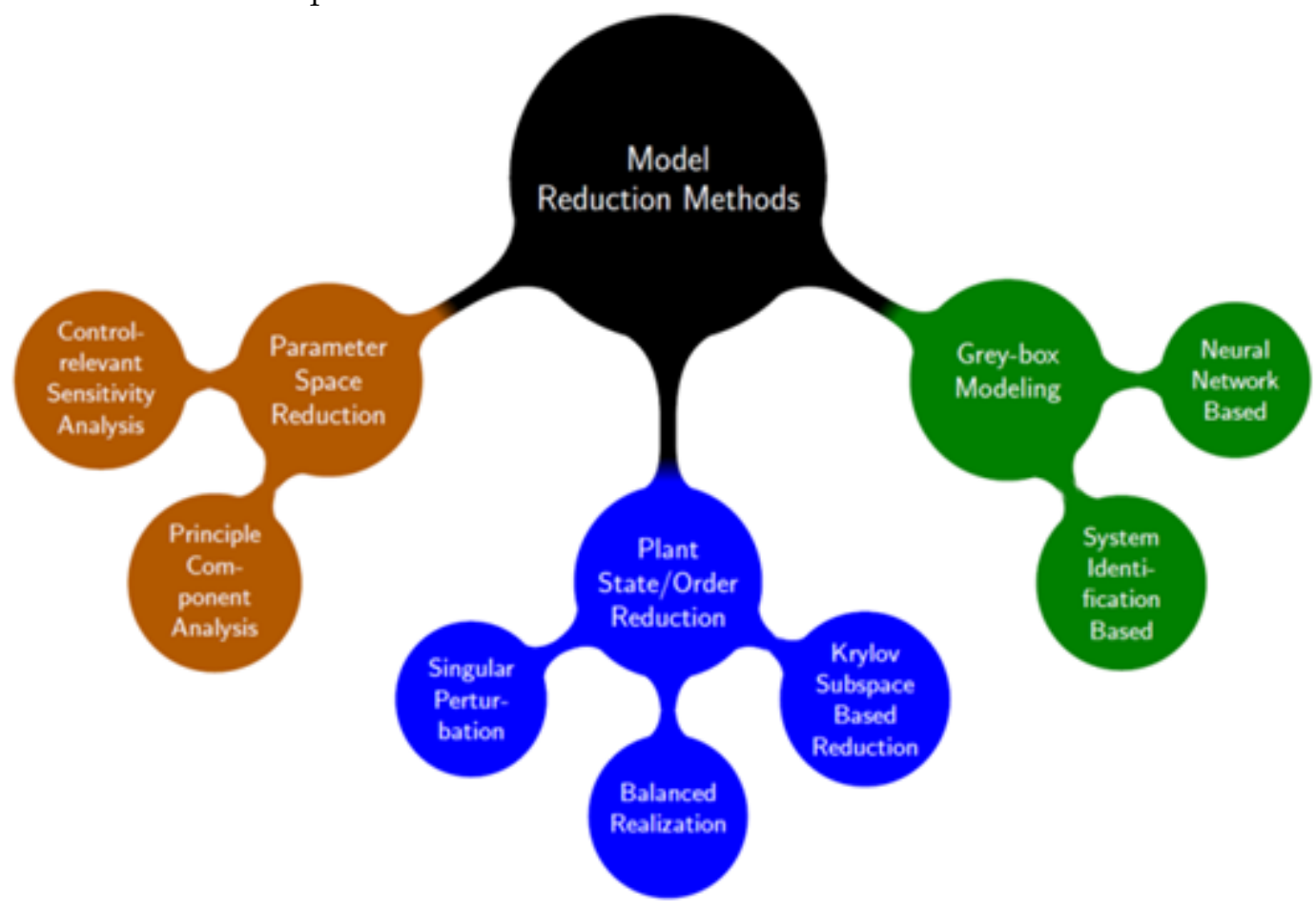

Figure 1.4: Overview of model reduction techniques for control systems.

\subsection{Dissertation Objectives}

The main objective of this study is developing an easily verifiable control theory framework that can be used to minimize controller software development time and cost by minimizing V\&V iterations. Specific project objectives are:

1. Developing easily verifiable control theories in continuous and discrete times,

2. Developing uncertainty prediction and propagation models for controller implementation imprecision, 
3. Developing control theory with robustness to implementation imprecisions,

4. Handling model uncertainties via an adaptive model-based controller design,

5. Design of a nontrivial processor-in-the-loop (PIL) and hardware-in-the-loop (HIL) platforms for real-time testing of the designed controllers against implementation uncertainties and modeling uncertainties,

6. Applying the theories of easily verifiable controller design for challenging automotive control problems.

\subsubsection{Dissertation Organization}

This $\mathrm{PhD}$ dissertation is organized as follows:

- Chapter 2 In this chapter, an early model-based methodology is presented to reduce the $\mathrm{V} \& \mathrm{~V}$ time. The application of the proposed methodology is demonstrated on a "Cold Start Emission" control problem in a mid-size passenger car. A nonlinear reduced order model-based controller based on singular perturbation approximation is designed to reduce cold start hydrocarbon emissions from a spark ignition (SI) combustion engine. A model-based simulation platform is created to verify the controller robustness against sampling, quantization and fixed-point arithmetic imprecision.

- Chapter 3 A model-based uncertainty-adaptive methodology is proposed in this chapter to enable easily verifiable controller design based on the formulation of continuous-time sliding mode controls (SMC). The proposed adaptive SMC 
improves the controller robustness against major implementation imprecisions including sampling and quantization. The application of the proposed technique is demonstrated on the engine cold start emission control problem. The cold start controller is first designed in a single-input single-output (SISO) structure with three separate sliding surfaces, and then is redesigned based on a multiinput multi-output (MIMO) SMC design technique using nonlinear balanced realization. The MIMO controller prioritizes the states of the model based on their energy and put more efforts to track trajectories with higher level of energy. The controller behavior is improved using the MIMO structure with fewer tunable parameters. The performance of the MIMO adaptive controller is validated in real-time by testing the control algorithm in a processor-in-theloop (PIL) platform.

- Chapter 4: In this chapter, a new discrete-time control approach is developed to mitigate the $\mathrm{ADC}$ imprecisions by (i) identifying the $\mathrm{ADC}$ imprecisions in the early stages of a controller design cycle, (ii) developing a mechanism for real-time prediction of uncertainties due to $\mathrm{ADC}$, and (iii) incorporating the predicted uncertainties into the controller design. To this end, a generic online technique is developed to predict sampling and quantization uncertainties on measured signals. Then a discrete sliding mode controller (DSMC) is designed for incorporation of ADC uncertainties. Inclusion of predicted imprecisions into the DSMC requires the knowledge of propagated uncertainties on control signals. An experimental approach is proposed for a general class of nonlinear 
systems to estimate the propagated ADC uncertainties on control inputs. The proposed control approach is illustrated on one linear and one nonlinear control problems, including DC motor and automotive engine controls. The designed controller is tested in real-time in the PIL setup using an actual ECU.

- Chapter 5 In this chapter, the new DSMC with predicted implementation imprecisions from Chapter 4 is extended to handle additive and multiplicative types of model uncertainties for linear and nonlinear systems using a discrete Lyapunov stability analysis. The proposed adaptive DSMC with predicted implementation imprecisions provides an integrated framework to improve the robustness of a common DSMC against both model and hardware (ADC) uncertainties. The performance of the proposed adaptive robust DSMC is evaluated on DC motor and nonlinear automotive engine control problems. Finally, the designed controllers are experimentally verified on a real ECU.

- Chapter 6: The implementation of the conventional continuous-time SMC on digital computers is limited, due to not only the ADC imprecisions, but also the chattering phenomena, which results in high frequency oscillations. One effective solution to minimize the chattering, and reduce the effects of data sampling and quantization is the use of higher order sliding modes. To this end, in this chapter, a new adaptive second order DSMC formulation is presented for a general class of MIMO uncertain nonlinear systems. Based on a Lyapunov stability argument and by invoking the new Invariance Principle, not only is the 
asymptotic stability of the controller guaranteed, but also the adaptation law is also derived to remove the uncertainties within the nonlinear plant dynamics. Moreover, a new switching control input is introduced to improve the controller robustness against data sampling and quantization imprecisions. The proposed controller is designed and experimentally tested in real-time for the linear DC motor, and the highly nonlinear automotive combustion engine case studies.

- Chapter 7 In this chapter, the generic DSMC with predicted ADC imprecisions from Chapter 4 is experimentally verified for an electronic throttle position tracking problem. Electronic throttle control is an integral part of an engine control unit that directly affects vehicle fuel economy, drivability, and engineout emissions by managing engine torque and air-fuel ratio through adjusting intake charge flow to the engine. The highly nonlinear dynamics of the throttle body call for nonlinear control techniques that can be implemented in real-time and are also robust to controller implementation imprecision. First, a nonlinear physical model for an electromechanical throttle body is derived. Parameters of the model are determined using techniques of model/parameter identification. Next, a DSMC is formulated for controlling the throttle position. The performance of the DSMC is examined under different sampling and quantization levels via the analog-to-digital converter.

Figure 1.5 summarizes the organization of chapters in this dissertation, along with publications from this thesis. 


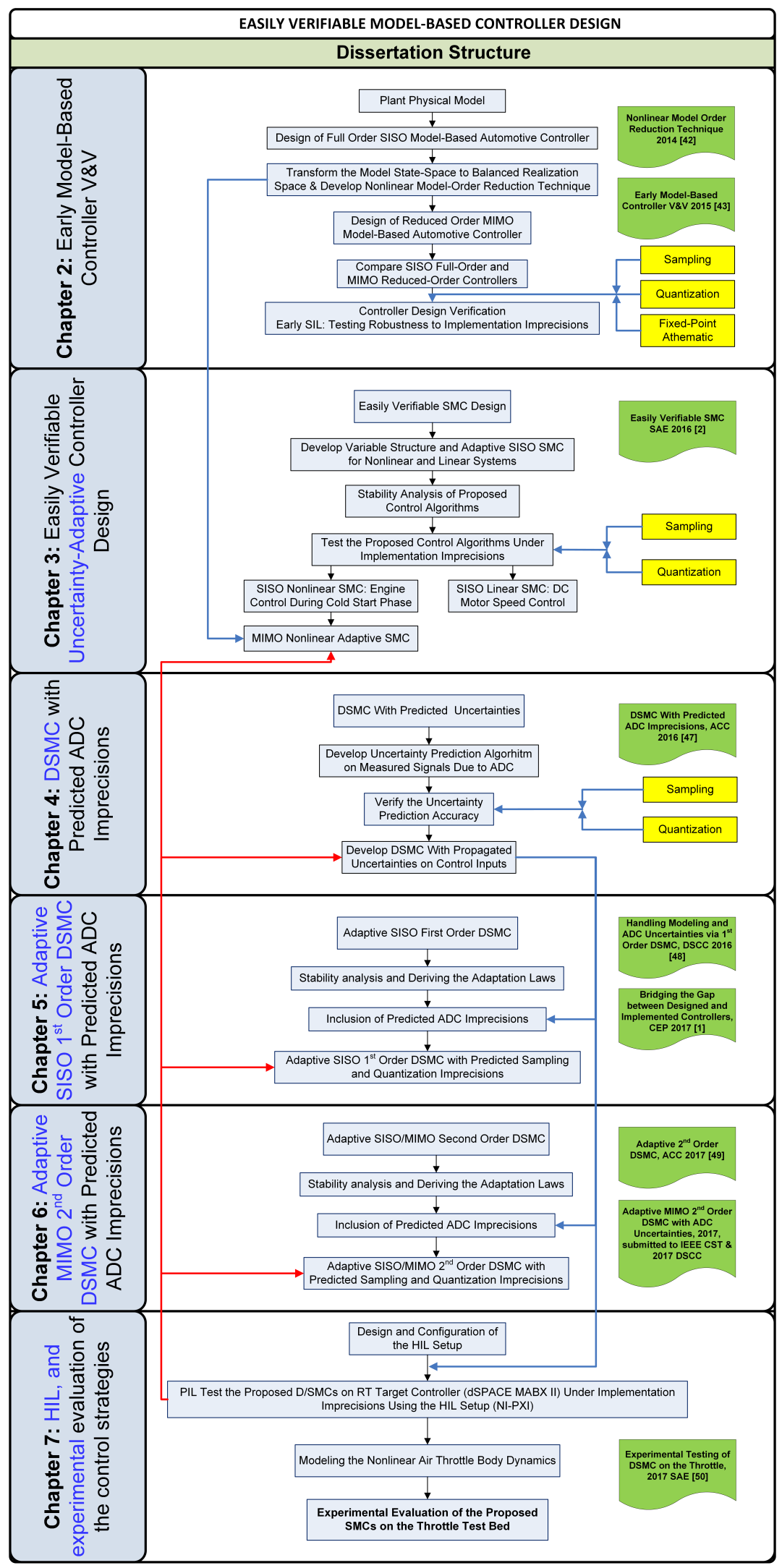

Figure 1.5: Organization of this dissertation. 


\section{Chapter 2}

\section{Early Model-Based Design and}

\section{Verification of Control Systems ${ }^{1}$}

\section{$2.1 \quad$ Introduction}

Controller software verification (CSV) is the critical process used to avoid mismatch between a designed and implemented controller. Imprecision caused by approximations in the process of implementing a controller's software is one of the main sources of error in the CSV of an automotive ECU. The software implementation process involves issues such as sampling, quantization, ECU scaling, fixed-point arithmetic,

\footnotetext{
${ }^{1}$ The contents of this chapter have been partially published in ASME Journal of Dynamic Systems, Measurement, and Control [1] (doi: 10.1115/1.4027845), and proceeding of 2014 SAE World Congress 2] (doi:10.4271/2014-01-1547). Reprinted with permissions by SAE @ 2017 SAE International and ASME as shown in Appendix D. Further distribution of these materials is not permitted without prior permissions from SAE and ASME.
} 
saturation, and operating system adjustments; all of these can cause mismatch between the original designed controller and the final implemented controller. The computational flow of a typical fixed-point automotive ECU is shown in Figure 2.1. Sampling, quantization and fixed-point arithmetic are the main sources of CSV errors.

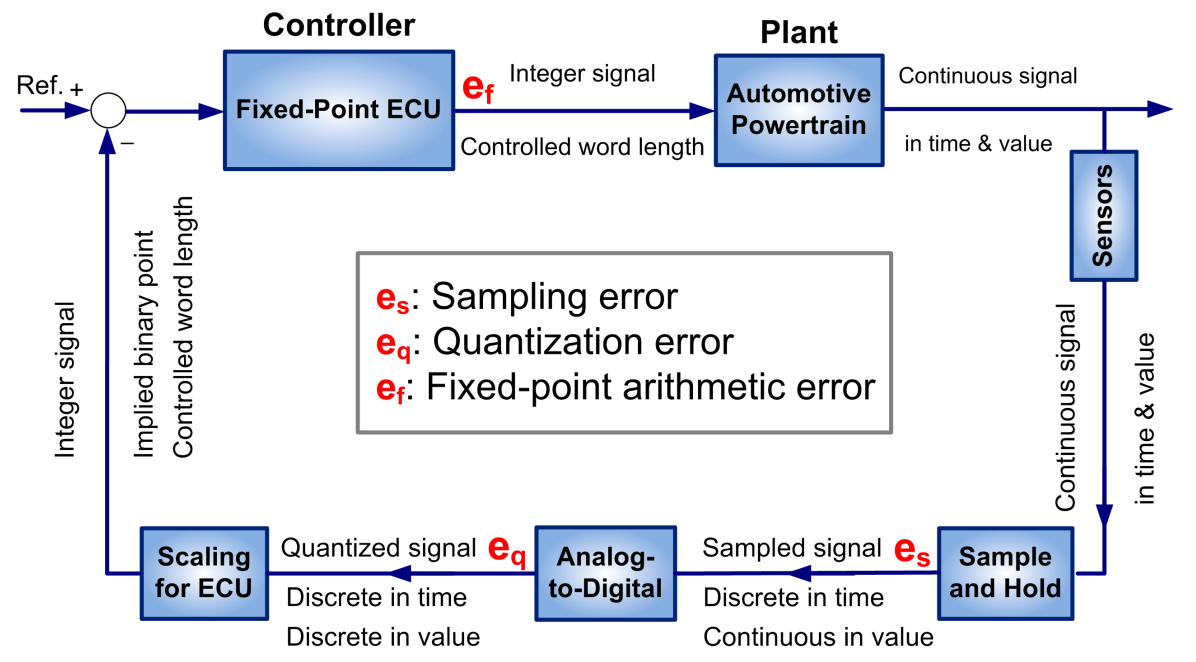

Figure 2.1: Computational flow in a typical fixed-point automotive control system.

A large number of errors detected during independent $\mathrm{V} \& \mathrm{~V}$ are introduced during the initial stages of a controller development and it will cost 10 times less if those errors are identified and fixed during the early stages of a controller software design [1]. Design and implementation of controllers involve three disciplines, including: control engineering, software engineering, and electronic hardware engineering. A critical gap is found when uncertainty in controller software/hardware implementation is not considered as part of the controller design.

Common CSV practice in the automotive industry is to test a controller after its 
software is fully implemented. This CSV practice is costly and time consuming. A number of studies have been conducted to improve CSV of complex embedded control systems such as automotive ECUs. Some of the recent studies include: (1) developing methods of model based software-in-the-loop [67, 68, 69, 70]; (2) automatic verification of a controller by stability analysis through using a model of implementation errors in a controller design [16, 71]; (3) developing techniques for verification of a controller's software [72]; (4) analytical methods to accurately compute a controller's reachable set, providing easier verification of a controller [73, 74, 75]; (5) developing tools for model-based verification of controllers [76, 77, 78]; (6) using advanced optimization solvers to find mathematically equivalent software implementation with smaller fixed-point arithmetic imprecision [79]; (7) model-based calibration of controllers to reduce $\mathrm{V} \& \mathrm{~V}$ required time [80, 81].

The contribution of this chapter is threefold. Firstly, an early model-based methodology is proposed for evaluating the controller performance under implementation imprecisions. This section presents the benefits of the proposed methodology for reducing $\mathrm{V} \& \mathrm{~V}$ efforts and reducing implementation requirements. Secondly, a new nonlinear model order reduction method is developed, based on singular perturbation approximation (SPA), to simplify the controller design for reducing V\&V. Thirdly, the performance results are illustrated by developing a nonlinear model-based controller to reduce cold start hydrocarbon (HC) emissions from an automotive engine. 


\subsection{Proposed CSV Methodology}

The proposed methodology and typical time-consuming process of $\mathrm{V} \& \mathrm{~V}$ for automotive controllers are shown in Figure 2.2. This study proposes an early model-based design and verification before launching the process of controller implementation, which will serve to reduce the time spent in the typical V\&V process. While using a minimum-order model-based controller will simplify the calibration and verification stages, the early $\mathrm{V} \& \mathrm{~V}$ can help identify and avoid the errors that will occur later in the controller implementation process.

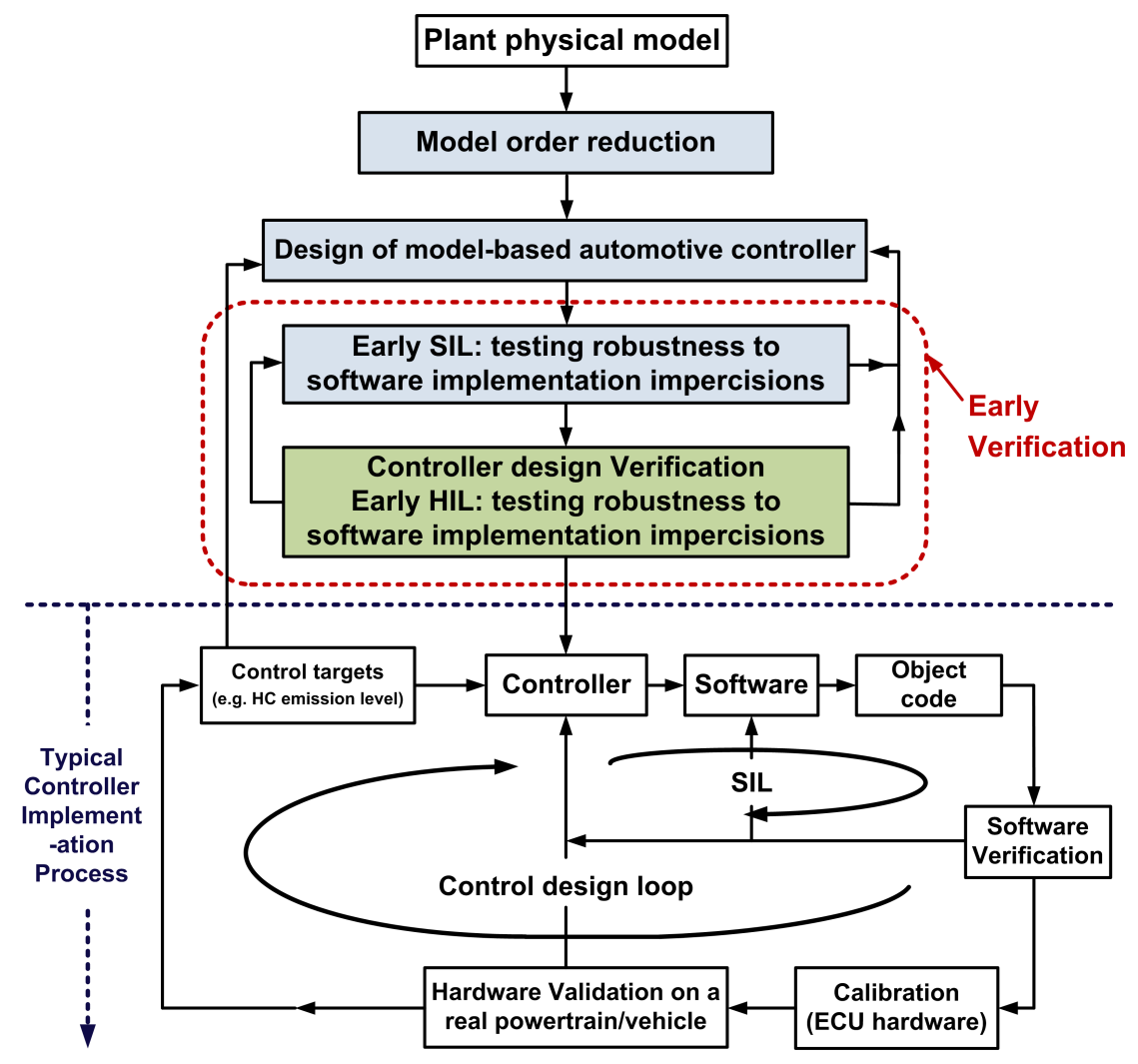

Figure 2.2: Proposed early model-based V \& V methodology. 
The proposed methodology in Figure 2.2 has two steps. The first step is developing a reduced order model for a controller design. This is particularly important given ever-increasing complexity in advanced automotive systems. Using a reduced order model is expected to reduce controller calibration load since a simplified model-based controller will be implemented. Reduced calibration efforts will lead to reduced V\&V time and efforts. To this end, developing model order reduction techniques is critical. Those techniques should be able to capture the most important part of the model's dynamics with minimum required states/parameters. The second step includes design of a model-based controller using a reduced order model. Next, the controller's robustness is evaluated against main imprecisions arising during the software implementation process. The robustness is determined by how sensitive the controller's performance is against imprecision caused by sampling rate, quantization level, and fixed-precision arithmetic in an ECU. The two steps in Figure 2.2 are discussed in the remaining of this section. The application of the proposed methodology is shown for a case study on automotive emission control.

\subsubsection{Nonlinear Model Order Reduction}

As proposed in Section 2.2, a plant model with a minimum order is desirable to simplify the design and verify the controller. The reduced order model must capture the most important part of the system's dynamics and guarantee acceptable estimation 
accuracy in comparison with its full order nonlinear model counterpart. In the remaining of this section, with respect to balanced realization and singular perturbation approximation techniques for linear systems, a novel nonlinear model order reduction methodology is presented.

\subsubsection{Balanced realization}

Linear balanced realization transfers the space of the physical states of the model into another linear space in which new non-physical states form a linear combination of the physical states. In this new space, controllability and observability gramians are diagonal and equal to the Hankel singular value (HSV) matrix. Since the magnitudes of HSVs indicate the level of each state's energy, significant differences among these singular values can be considered as a proof of the state's dominance [64].

For the linear systems, the HSVs of the system are defined as the square root of the eigenvalues of $P Q$, where $P$ and $Q$ are the observability and controllability gramians of the linearized system, respectively:

$$
\sigma_{i}=\sqrt{\lambda_{i}(P Q)} \quad i=1, \ldots, n
$$

where, $\lambda_{i}$ is the $P Q$ eigenvalue. The HSVs values are then placed in descending order into the HSV matrix $(\Sigma)$. A similarity transformation matrix is then determined such that the transformed gramians are diagonal and equal to $\Sigma$. If the linear system is observable, there exists a positive definite square root matrix $(R)$ for the observability 
gramian. A Hermitian eigenvalue decomposition on $R P R^{\top}$ is then performed to find the unitary matrix $W$, where $R^{\top}$ indicates the transpose of matrix $R$. The similarity transformation matrix that achieves a balanced realization can then be calculated.

$$
\begin{gathered}
Q=R R^{\top} \\
R P R^{\top}=W \Sigma^{2} W^{\top} \\
T=R^{-1} W \Sigma^{1 / 2}
\end{gathered}
$$

The balanced state-space realization is calculated using the transformation $x=T z$. $T$ is defined as the coordinate transition matrix which transfers the states of the physical model $(X)$ to another non-physical space $(Z)$ where the system is balanced. It must be noted that dimensions of $X$ and $Z$ are still equal.

\subsubsection{Singular Perturbation Approximation}

The linear singular perturbation approximation (SPA) technique simplifies the dynamics of the linear system with respect to differences among HSVs of the balanced realization form of a linear system [65]. The idea of linear balanced realization is used to extend the linear SPA order reduction method to nonlinear systems. Consider a general nonlinear system: 


$$
\left\{\begin{array}{l}
\dot{x}=f(x, u) \\
y=h(x, u)
\end{array}\right.
$$

where $x \subset X \in \mathbb{R}^{n}, y \subset Y \in \mathbb{R}^{m}$ and $u \subset U \in \mathbb{R}^{h}$ are the state, the output and the input vectors, respectively. Once linearzed realization of the nonlinear system (Eq. (2.5) ) is obtained, the coordinate transition matrix can be calculated by a similar approach using (Eq. (2.4)). Eventually, state-space transition will be as follows:

$$
x_{(n \times 1)}=T_{(n \times n)} z_{(n \times 1)}, \dot{x}_{(n \times 1)}=T_{(n \times n)} \dot{z}_{(n \times 1)}
$$

by applying Eq. (2.6) transformation on nonlinear system Eq. (2.5), non-physical states are calculated by:

$$
\left\{\begin{array}{l}
\dot{z}=T^{-1} f(T z, u) \\
y=h(T z, u)
\end{array}\right.
$$

Principle of SPA order reduction is based on dividing the dynamics into the fast and the slow parts. To separate the fast and the slow parts based on the HSVs, Eq. (2.6) is rearranged as follows:

$$
\dot{x}_{(n \times 1)}=\left[\begin{array}{c}
\dot{x}_{s} \\
\dot{x}_{f}
\end{array}\right]=T \dot{z}_{(n \times 1)}=T\left[\begin{array}{c}
\dot{z}_{s} \\
\dot{z}_{f}
\end{array}\right]
$$

where $\dot{x}_{s} \in \mathbb{R}^{r}$ and $\dot{x}_{f} \in \mathbb{R}^{(n-r)}$ are the slow and the fast state-space vectors of $X \in \mathbb{R}^{n}$ 
space, respectively. Additionally, $\dot{z}_{s} \in \mathbb{R}^{r}$ and $\dot{z}_{f} \in \mathbb{R}^{(n-r)}$ are the state-space vectors of $Z \in \mathbb{R}^{n}$ space corresponding to $\dot{x}_{s}$ and $\dot{x}_{f}$, respectively. Considering the lengths of slow and the fast state-space vectors and also descending order of HSVs elements in $\Sigma$, the coordinate transition matrix $T$ can be partitioned as follows:

$$
\begin{gathered}
T=\left[\begin{array}{cc}
T_{11(r \times r)} & T_{12(r \times(n-r))} \\
T_{21((n-r) \times r)} & T_{22((n-r) \times(n-r))}
\end{array}\right] \\
T^{-1}=\left[\begin{array}{cc}
T_{11}^{-1}{ }_{(r \times r)} & T_{12}^{-1}(r \times(n-r)) \\
T_{21}^{-1}{ }_{((n-r) \times r)} & T_{22}^{-1}{ }_{((n-r) \times(n-r))}
\end{array}\right]
\end{gathered}
$$

The fast part of the dynamics diminishes and reaches its steady state conditions prior to the slow part; thus, its derivative can be set to zero. By substituting Eq. (2.9) in Eq. (2.8) and setting $\dot{x}_{f}$ to zero, we have:

$$
\dot{x}=\left[\begin{array}{c}
\dot{x}_{s} \\
0
\end{array}\right]=\left[\begin{array}{ll}
T_{11} & T_{12} \\
T_{21} & T_{22}
\end{array}\right]\left[\begin{array}{c}
\dot{z}_{s} \\
\dot{z}_{f}
\end{array}\right]
$$

then, Eq. 2.11 can be simplified into the following representation:

$$
\dot{x}_{s} \cong\left(T_{11}-T_{12} T_{22}^{-1} T_{21}\right) \dot{z}_{s}
$$

We define $T_{S P A}$ as follows:

$$
T_{S P A}=T_{11}-T_{12} T_{22}^{-1} T_{21}
$$


thus:

$$
x_{s} \cong T_{S P A} z_{s}
$$

In addition, in a similar way it can be shown that:

$$
\begin{aligned}
& \dot{z}_{s} \cong T_{S P A}{ }^{*} \dot{x}_{s} \\
& z_{s} \cong T_{S P A}{ }^{*} x_{s}
\end{aligned}
$$

where:

$$
T_{S P A}{ }^{*} \cong T_{11}{ }^{-1}-T_{12}{ }^{-1}\left(T_{22}{ }^{-1}\right)^{-1} T_{21}{ }^{-1}
$$

Slow and energetic states $\left(z_{s} \in \mathbb{R}^{r}\right)$ of the $Z \in \mathbb{R}^{n}$ space are calculated by applying Eq. (2.16) transformation to the slow part of $x_{s} \in \mathbb{R}^{r}$ states in $X \in \mathbb{R}^{n}$ space. These $z_{s}$ states constitute the $Z_{s} \in \mathbb{R}^{r}$ reduced non-physical sub-space $\left(Z_{s} \subset Z\right)$. The objective of the model order reduction approach is calculating the states of $Z_{s}$ space directly from $X$ space's states. However, $T_{S P A} \in \mathbb{R}^{r \times r}$ and $T_{S P A}{ }^{*} \in \mathbb{R}^{r \times r}$ matrices are square. Therefore, to transit from a higher order space $(X)$ to a lower order space $\left(Z_{s}\right)$, these matrices should be modified in dimensions. In transition from $n^{\text {th }}(r<n)$ order space to lower $r^{\text {th }}$ order space, according to Eq. 2.14), the coordinate transition matrix should be a $(n \times r)$ matrix. In addition, the coordinate transition matrix from a lower space to a higher order space, Eq. (2.16), should be $(r \times n)$. Regarding the dimension of $T_{S P A}$ and $T_{S P A}{ }^{*}$, to compensate for the dimension of these two matrices, 
two adjoint matrices are associated with them $\left(T_{c}\right.$ and $\left.T_{c}{ }^{*}\right)$ :

$$
\begin{gathered}
x_{(n \times 1)} \cong\left[\begin{array}{c}
T_{S P A(r \times r)} \\
T_{c((n-r) \times r)}
\end{array}\right]_{(n \times r)} z_{s(r \times 1)} \\
z_{s(r \times 1)} \cong\left[T_{\left.S P A^{*}{ }_{(r \times r)} \mid T_{c}{ }_{(r \times(n-r))}\right]_{(r \times n)} x_{(n \times 1)}}\right.
\end{gathered}
$$

The simplest choice for these adjoint matrices is the null matrix. Eq. (2.18) and (2.19) are simplified in the following general representations:

$$
\begin{aligned}
& x_{(n \times 1)} \cong \tilde{T}_{S P A_{(n \times r)}} z_{s(r \times 1)} \\
& z_{s(r \times 1)} \cong \tilde{T}_{S P A_{(r \times n)}}^{*} x_{(n \times 1)}
\end{aligned}
$$

where, $\tilde{T}_{S P A}$ and $\tilde{T}_{S P A}^{*}$ are state transition matrices between higher order space $(X)$ and non-physical reduced order space $\left(Z_{s}\right)$ :

$$
\begin{gathered}
\tilde{T}_{S P A}=\left[\begin{array}{c}
T_{S P A} \\
- \\
T_{c}
\end{array}\right] \\
\tilde{T}_{S P A}^{*}=\left[T_{S P A}^{*} \mid T_{c}^{*}\right]
\end{gathered}
$$


Once the state transition matrix will be reduced by using singular perturbation approximations (Eq. (2.22) and (2.23)), the SPA balanced reduction method can be extended to a nonlinear system. By applying SPA to the nonlinear system Eq. 2.5, we have a SPA nonlinear reduced order model with respect to Eq. (2.7) as follows:

$$
\left\{\begin{array}{l}
\dot{z}_{s} \cong \tilde{T}_{S P A}^{-1} f\left(\tilde{T}_{S P A} z_{s}, u\right) \\
\tilde{y} \cong h\left(\tilde{T}_{S P A} z_{s}, u\right)
\end{array}\right.
$$

where, $\tilde{y}$ is the estimated output of the nonlinear SPA reduced model and $\tilde{T}_{S P A}^{*} \cong$ $\tilde{T}_{S P A}^{-1}$. It can be concluded from Eq. 2.12-2.17 that the reduced order of $T_{S P A}$ and $T_{S P A}{ }^{*}$ matrices contain all elements of the coordinate state transition matrix $\left(T_{11}, T_{12}, T_{21}, T_{22}\right)$ and its inverses. Thus, no part of the original dynamics will be ignored during the reduction. The chart in Figure 2.3 shows the roadmap for developing reduced SPA nonlinear model with respect to physical model and balanced realization form of equations.

\subsubsection{Controller Robustness Evaluation against Implementa- tion Imprecision}

As shown in Figure 2.1, there are three sources of implementation errors, including data sampling, quantization, and fixed-point data type conversion imprecisions. In order to evaluate the robustness of a model-based controller design, the impact of 


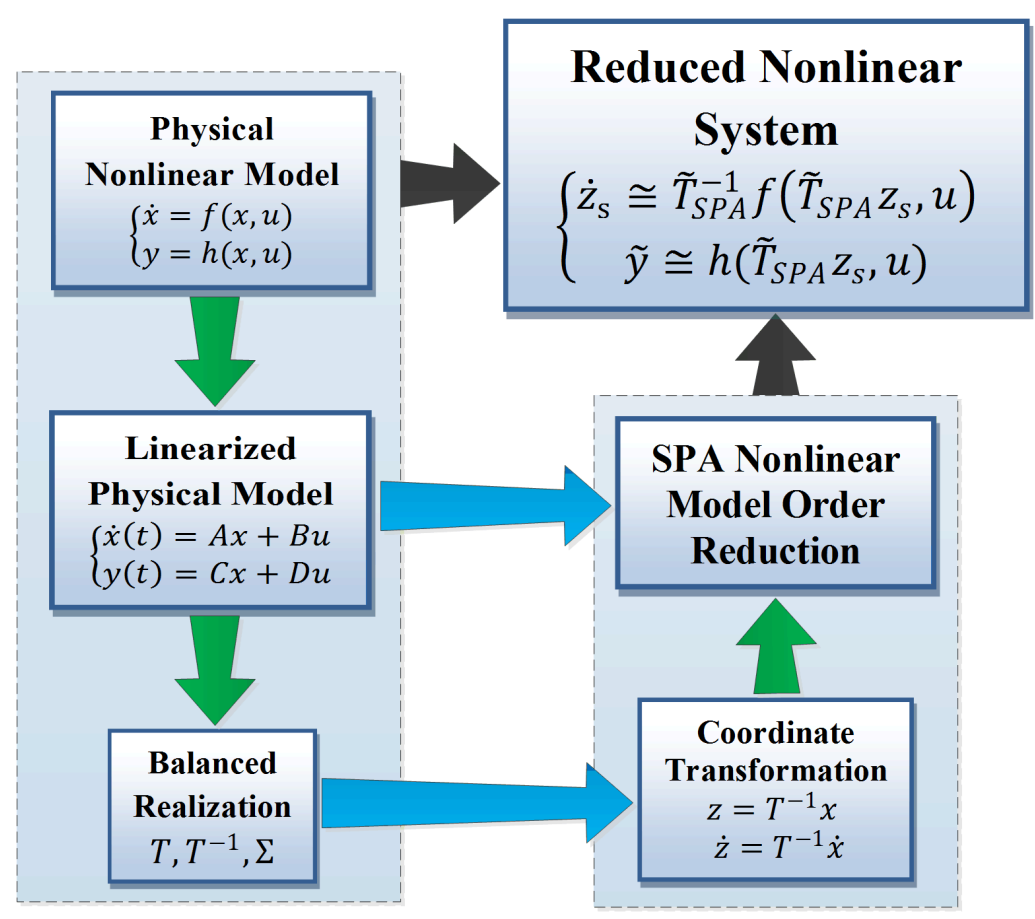

Figure 2.3: Process of nonlinear SPA model order reduction.

these imprecisions are investigated during the second step of the proposed early modelbased CSV approach shown in Figure 2.2. A fixed-point data is a triple $(s, n, m)$ characterized by a sign bit $(s)$, word length/number of bits $(n)$ and a fraction part $(m)$ :

$$
\begin{cases}\frac{1}{2^{m}} \sum_{i=0}^{n-1} 2^{i} b_{i} & \text { (a) Unsigned } \\ \frac{1}{2^{m}}\left[-2^{n-1} b_{n-1}+\sum_{i=0}^{n-1} 2^{i} b_{i}\right] & \text { (b) Signed }\end{cases}
$$

where, $b$ is the bit vector representing values between $-2^{n-1}$ and $2^{n-1}-1$ for signed fixed-point data and representing values between 0 and $2^{n}-1$ for unsigned fixed-point data. The fixed-point data represents an approximation of a real number. The associated imprecision from this approximation is considered as fixed-point imprecision 
$\left(e_{f p}\right)$ which is less than $\frac{1}{2^{m}}$ for the representation of a real number. The total $e_{f p}$ depends on arithmetic operations performed inside the controller. The $e_{f p}$ directly affects the accuracy of the controller outputs.

The ECU communicates with signals discretized in both time, through sampling, and in value, through A-to-D converters (ADCs). The sampling rate determines the time discretization resolution, whereas the quantization level in the ADCs determines the resolution in value. These data resolutions will affect the performance of the ECU. Sampling imprecision $\left(e_{s}\right)$ is calculated by:

$$
e_{s} \leq|\vartheta| T_{s}
$$

where, $T_{s}$ is sampling time and $\vartheta$ is the maximum negative/positive slopes of signals. Quantization rounding imprecision $\left(e_{q}\right)$ is determined by:

$$
-\frac{1}{2} \frac{F S R}{2^{n}}<e_{q}<\frac{1}{2} \frac{F S R}{2^{n}}
$$

where, $n$ is the number of bits and $F S R$ is the full-scale range of signal. In the next section, first the proposed nonlinear model order reduction technique in Section 2.2.1 is applied to design a reduced order controller for a combustion engine cold start emission model. Next, in Section 2.3.4.1, the robustness of the designed controller will be studied under implementation imprecisions. 


\subsection{Case Study: Cold Start Emission Control}

Cold start emissions account for over $80 \%$ of the total $\mathrm{HC}$ emissions in standard driving cycles [82, 83]. Poor air-fuel mixture formation and low efficiency of the exhaust

catalytic converters are two main causes of high emission levels of the passenger cars during the cold start operation. The critical cold phase is the 2-5 minute time period between the cold start and the moment the catalytic converter reaches its operating temperature (i.e. $200-300^{\circ} \mathrm{C}$ ). Cold start engine controller design is a well recognized challenge with increasing importance in moving towards green vehicles. This challenge is mainly due to highly nonlinear behavior of the automotive engines during cold start. In addition, during the cold start phase, reliable data from Air-Fuel Ratio (AFR) and exhaust temperature sensors are not available.

\subsubsection{Cold start emission model}

A physical model from [84] is used to estimate tailpipe HC emissions during cold start. The model is parameterized for a 2.4-liter, 4-cylinder, DOHC 16 valve Toyota 2AZFE engine and a three-way catalyst exhaust aftertreatment system. The engine rated power is $117 \mathrm{~kW} @ 5600 \mathrm{RPM}$ and it has a peak torque of $220 \mathrm{Nm} @ 4000 \mathrm{RPM}$. The experimental validation of different components of the model is found in [7, 85, 86]. The inputs to the model are $\dot{m}_{a i}$, air mass flow rate into the intake manifold, $\dot{m}_{f c}$, commanded fuel mass flow rate, and $\Delta$, spark timing after top dead center (ATDC). The model includes five states, indicated with the following dynamic equations: 


$$
\begin{gathered}
\dot{x}_{1}=\dot{m}_{a}=\dot{m}_{a i}-\dot{m}_{a o}\left(m_{a}, \omega_{e}\right)=f_{m_{a}} \\
\dot{x}_{2}=\dot{\omega}_{e}=\frac{1}{J}\left[T_{E}\left(m_{a}, \omega_{e}\right)\right]=f_{\omega_{e}} \\
\dot{x}_{3}=\ddot{m}_{f}=\frac{1}{\tau_{f}}\left[\dot{m}_{f c}-\dot{m}_{f}\right]=f_{\dot{m}_{f}} \\
\dot{x}_{4}=\dot{T}_{c a t}=\frac{1}{m C_{p}}\left[\dot{Q}_{g e n}+\dot{Q}_{\text {in }}-\dot{Q}_{o u t}\right]=f_{T_{c a t}} \\
\dot{x}_{5}=\dot{T}_{e x h}=\frac{1}{\tau_{e}}\left[S I(\Delta) \cdot A I\left(m_{a}, \omega_{e}, \dot{m}_{f}\right)-T_{e x h}\right]=f_{T_{e x h}}
\end{gathered}
$$

The five states are the mass of air inside the intake manifold $\left(m_{a}\right)$, the engine speed $\left(\omega_{e}\right)$, fuel mass flow rate into the cylinders $\left(\dot{m}_{f}\right)$, the catalyst temperature $\left(T_{\text {cat }}\right)$, and the exhaust gas temperature $\left(T_{e x h}\right)$. Details of the functions and constants in Eq. 2.28 to 2.32 are found in the Appendix A. The HC production rate from the engine $\left(\dot{H} C_{\text {eng }}\right)$ is calculated along with the catalytic converter efficiency $\left(\eta_{\text {cat }}\right)$ to give the $\mathrm{HC}$ emission rate out of the tailpipe $\left(\dot{H} C_{t p}\right)$.

$$
\begin{aligned}
& \dot{H} C_{e n g}=\dot{m}_{f} \frac{r_{c}-1}{r_{c}} \exp \left[-a\left[\frac{\theta_{e v o}-\theta_{0}(\Delta)}{\delta \theta\left(m_{a}, \omega_{e}, \dot{m}_{f}\right)}\right]^{n}\right] \\
& \eta_{\text {cat }}=0.98\left(1-\exp \left[-5\left(\frac{\frac{A F R}{A F R_{s t}}-0.7}{0.3}\right)^{15}\right]\right) \\
& \left(1-\exp \left[-0.2\left(\frac{T_{c a t}-30}{150}\right)^{5}\right]\right) \\
& \dot{H} C_{t p}=\dot{H} C_{e n g}\left(1-\eta_{c a t}\right)
\end{aligned}
$$


Where, $\mathrm{AFR}_{s t}$ is the AFR at stoichiometric condition and $r_{c}$ is the compression ratio. $\delta \theta, \theta_{0}$ and $\theta_{\text {evo }}$ are fuel burn rate function and parameters which are detailed in the Appendix A. Eq. (2.28) to 2.35 altogether shape the dynamical system equations in which the output of the model is the tailpipe $\mathrm{HC}$ production rate. The difference between engine-out and tailpipe $\mathrm{HC}$ emission depends on the catalytic converter performance in the exhaust system. Eventually, cumulative $\mathrm{HC}$ generated during the cold phase $\left(H C_{c u m}\right)$ is calculated by:

$$
H C_{c u m}=\int \dot{H} C_{t p} d t
$$

The cold start model was validated with the experimental data from the same engine in [7]. A comparison between measured and simulated $H C_{c u m}$ emission is shown in

Figure 2.4. It is observed that at the end of the simulation period (200 seconds), there is less than $2 \%$ error between measured and simulated $H C_{\text {cum }}$ results.

\subsubsection{Reduced Order Engine Model/Controller}

Due to strong nonlinearity of the engine cold start model, linear model order reduction technique can not accurately estimate the response of the engine model. Therefore, nonlinear model order reduction should be applied to the system's dynamics. To this end, the nonlinear model order reduction technique from Section 2.2.1 is used to design a reduced order cold start emission model and controller. 


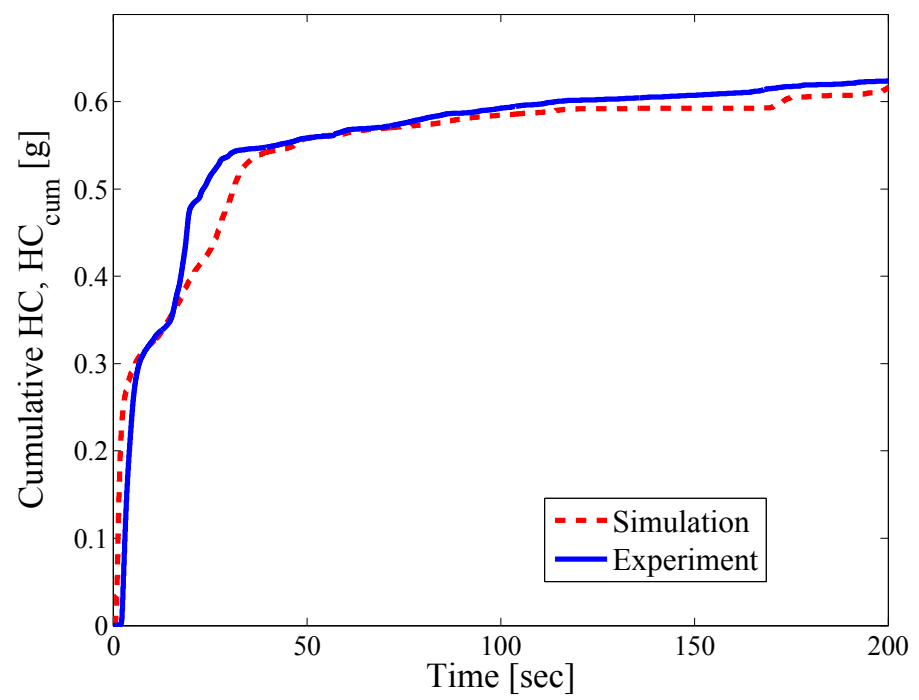

Figure 2.4: Comparison between measured and simulated cumulative tailpipe HC emission [7].

Cold start model order reduction is done in two main steps with respect to the methodology shown in Figure 2.3. In the first step, a balanced realization approach is used to reduce the order of the cold start model. Balanced realization is a transformation of the original states of a system into new states consisting of linear combinations of the original states such that the controllability and observability gramians are equal and diagonal. In the second step, the results from balanced realization are used and a nonlinear singular perturbation approximation (SPA) technique is developed. In SPA, fast and slow states of the system dynamics are separated. Then, slow and more energetic states will be selected as a reduced representation of the nonlinear model.

In order to calculate the balanced realization form of the engine cold start model, the nonlinear model is first linearized around an operation point. Linearization of the 
nonlinear model is done using the first order approximation of Taylor expansion with respect to a certain operation point before the catalyst light-off point. The linearized engine cold start model can be expressed in a general linear state-space realization as follows:

$$
\left[\begin{array}{c}
\dot{x} \\
y
\end{array}\right]=\left[\begin{array}{ll}
A_{(5 \times 5)} & B_{(5 \times 3)} \\
C_{(1 \times 5)} & D_{(1 \times 3)}
\end{array}\right]\left[\begin{array}{l}
x \\
u
\end{array}\right]
$$

where,

$$
\begin{aligned}
& x=\left[\begin{array}{lllll}
m_{a} & \omega_{e} & \dot{m}_{f} & T_{c a t} & T_{e x h}
\end{array}\right]^{T} \\
& u=\left[\begin{array}{lll}
\dot{m}_{a i} & \dot{m}_{f c} & \Delta
\end{array}\right]^{T} \\
& y=\dot{H} C_{t p}
\end{aligned}
$$

In the case of the linearized engine cold start model (Eq. (2.37), five HSVs are calculated using Eq. (2.1). The values are then placed in descending order into the HSV matrix $(\Sigma)$ :

$$
\Sigma=\operatorname{diag}\left[\sigma_{1}, \sigma_{2}, \sigma_{3}, \sigma_{4}, \sigma_{5}\right]
$$

where $\sigma_{1} \geq \sigma_{2} \geq \sigma_{3} \geq \sigma_{4} \geq \sigma_{5}$.

Here, with respect to Eq. (2.24), the order of the 5-state cold start model is reduced 
using proposed nonlinear SPA technique based on the balanced realization. The resulting HSV values from Eq. (2.41) are plotted in Figure 2.5. It is observed that there is a significant difference between orders of HSVs for the first three states and the orders of HSVs for the last two states. Thus, the new states in $Z$ space are mainly functions of $f_{m_{a}}, f_{\omega_{e}}$, and $f_{\dot{m}_{f}}$. The resulting reduced cold start model has three new non-physical states which are realizations of the slow states in $Z_{\text {s }}$ space:

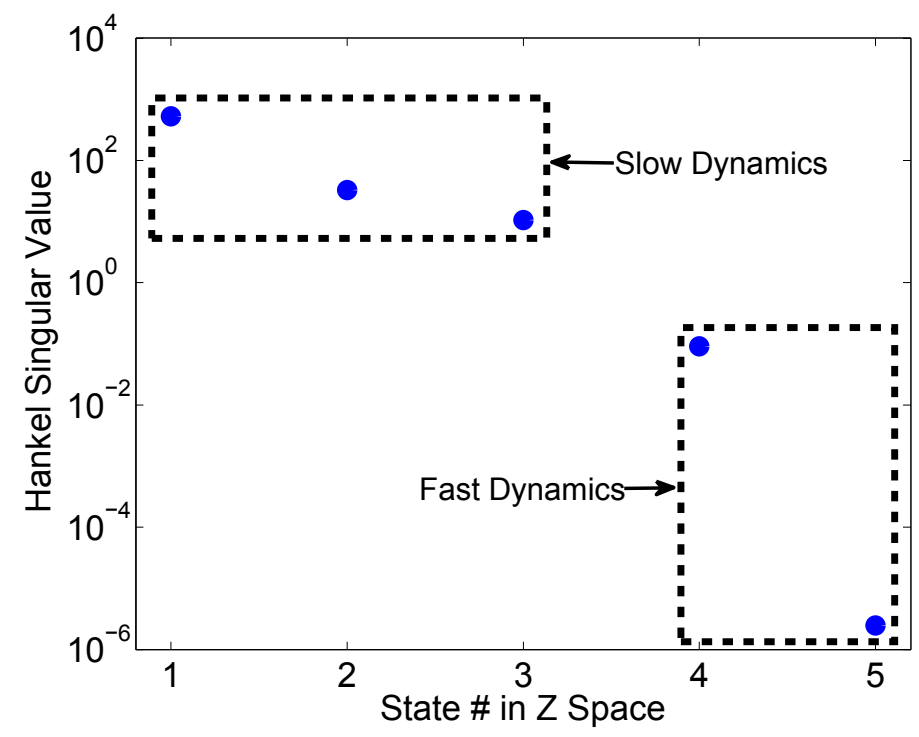

Figure 2.5: Hankel singular values for cold start emission model.

$$
\dot{z}_{s(3 \times 1)} \cong \tilde{T}_{S P A_{(3 \times 5)}}^{-1} F_{(5 \times 1)}
$$

where, $F$ is a vector including the original nonlinear state functions in $X$ space.

$$
F=\left[\begin{array}{lllll}
f_{m_{a}} & f_{\omega_{e}} & f_{\dot{m}_{f}} & f_{T_{c a t}} & f_{T_{e x h}}
\end{array}\right]^{T}
$$


(a)

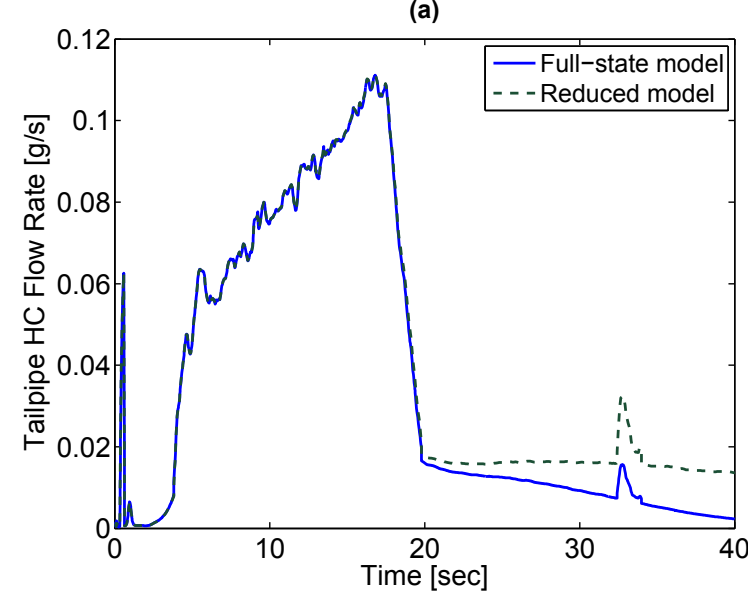

(b)

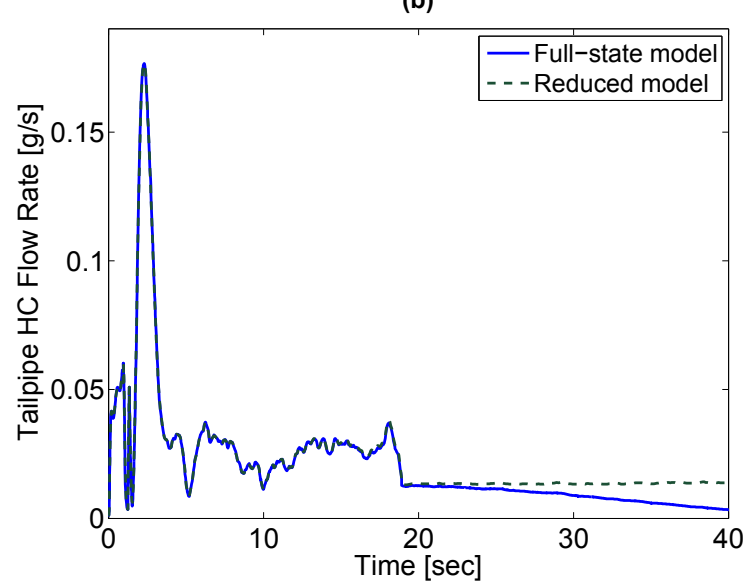

Figure 2.6: Comparison of tailpipe HC flow rates predictions by full state and reduced models.

Performance of the nonlinear SPA order reduction method is evaluated by testing the technique on the original cold start nonlinear model. Open loop control signals are needed to simulate the reduced order nonlinear model. Two sets of real data, which are taken from cold start operation of the Toyota engine, are used to validate the reduced model. In each set, three input signals, $\dot{m}_{a i}, \dot{m}_{f c}$, and $\Delta$, are used to run the simulation on the original nonlinear model and the reduced order model. Figure 2.6 shows the comparison between $\mathrm{HC}$ tailpipe flow rates from the full and reduced order nonlinear models for the two sets of real data.

The results show that the reduced nonlinear SPA model captures the main dynamics of the tailpipe $\mathrm{HC}$ during the first 40 seconds. In a modern vehicle the catalyst lightoff typically occurs before 40 seconds; thus, the cold start control strategy is critical during the very early seconds of the engine operation where the reduced SPA model is shown to be accurate. In the next section, the reduced-order nonlinear SPA model 
is used to design a SMC for reducing the cold start tailpipe $\mathrm{HC}$ emissions.

\subsubsection{Controller Design}

The main control objective is to minimize $H C_{c u m}$. This objective requires not only reducing raw $\dot{H} C_{\text {eng }}$ but also quickly heating up catalyst to shorten the catalyst lightoff period. AFR and $\omega_{e}$ influence both $\dot{H C_{e n g}}$ and $T_{c a t}$, while $T_{\text {exh }}$ directly influences $T_{\text {cat }}$. Thus three control trajectories $\left(\mathrm{AFR}_{d}, \omega_{e, d}\right.$, and $\left.\mathrm{T}_{\text {exh,d }}\right)$ are defined to minimize $H C_{c u m}$, as shown in Figure 2.7. $\mathrm{AFR}_{d}$ and $\omega_{e, d}$ control trajectories are taken from the engine control unit of the Toyota 2AZ-FE engine. The exhaust temperature of $650^{\circ} \mathrm{C}$ is chosen for $\mathrm{T}_{e x h, d}$ using available experimental data for the engine [86].

The controller adjusts the inputs to the engine plant to control the engine tailpipe HC emission. As shown in Figure 2.7, there are three sub-controllers to track each of desired trajectories. The first sub-controller adjusts fuel injection rate through tracking the desired AFR trajectory. The second sub-controller is the idle speed controller which impacts the exhaust gas flow rate for heating the catalyst through tracking a desired idle speed trajectory. The third sub-controller adjusts spark timing which impacts the exhaust gas temperature through tracking the desired exhaust gas temperature. Spark timing is often delayed for the cold start phase since a delayed ignition leads to higher exhaust gas temperature. However, delayed spark timing can lead to high engine-out HC emission. 


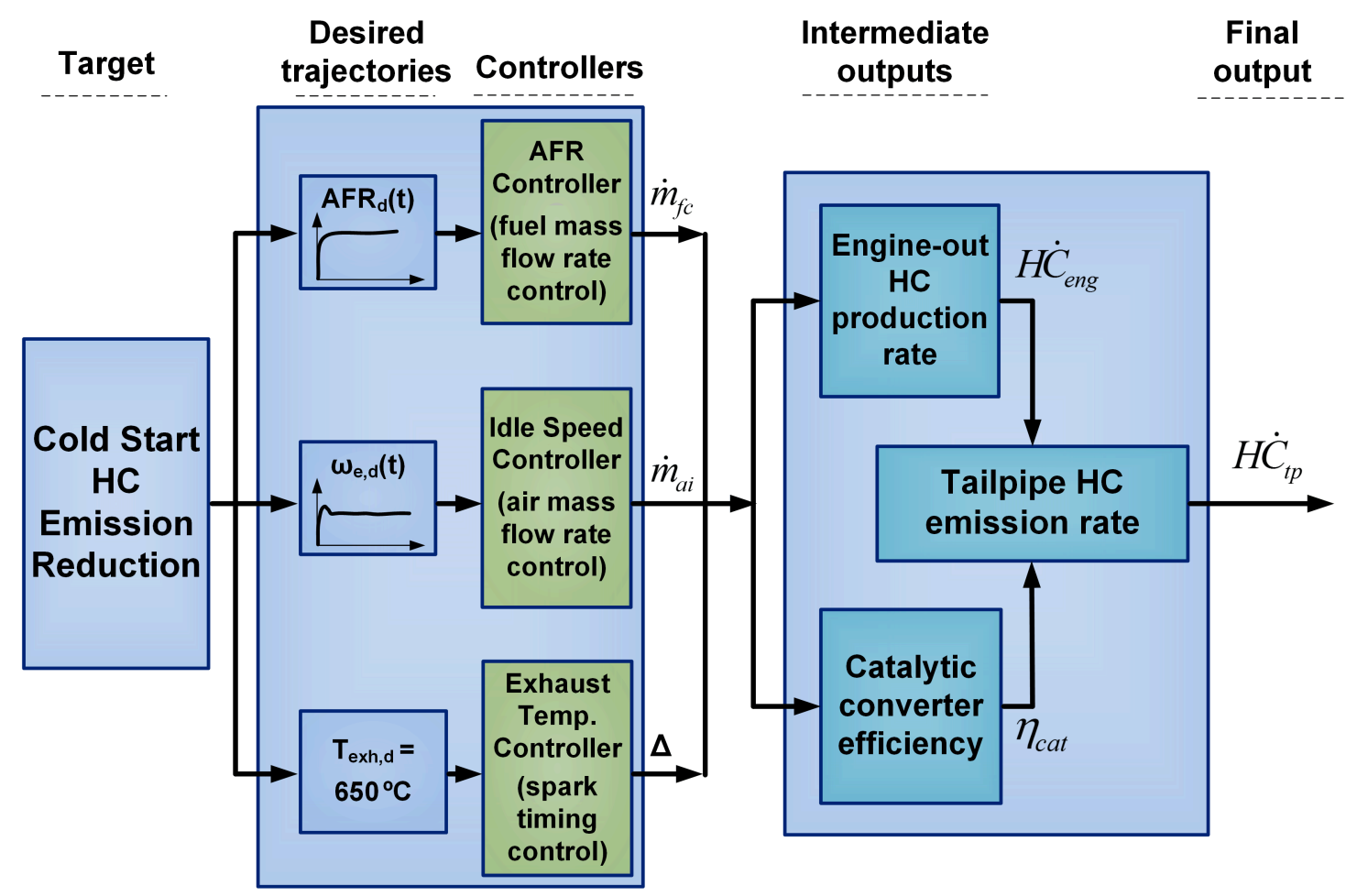

Figure 2.7: Structure of the designed cold start controller.

A proper choice for tracking the three desired trajectories is a SMC which ensures obtaining the desired $\mathrm{AFR}_{d}, \mathrm{~T}_{e x h, d}$, and $\omega_{e, d}$ trajectories. The desired trajectories in the reduced space are mixed functions of the reduced states. Thus, they cannot be controlled just by one of the control inputs. In other words, SMC based on the reduced model is a Multi-Input Multi-Output (MIMO) controller.

Before addressing the design of the MIMO SMC, it must be mentioned that, as can be seen from model equations (Eq. (2.28)-(2.32), idle speed has no direct input. Therefore a fourth input, $\mathrm{m}_{a, d}$, is defined synthetically and represents the desired mass of air in the intake manifold. Four sliding surfaces in physical space are used 
to track the engine states using these four inputs. We define $\Psi$ as the vector of the control inputs:

$$
\Psi=\left[\begin{array}{c}
m_{a} \\
\omega_{e} \\
\dot{m}_{f} \\
T_{e x h}
\end{array}\right]
$$

Comparing Eq. (2.44) with model states shows that the vector of control inputs consists of all states except for $T_{\text {cat }}$. Therefore, we define a state transition matrix, $\tilde{T}_{p}$, which is part of $T$ in which column associated with $T_{\text {cat }}$ is taken away. The reduced order SPA engine cold start model is used to design a MIMO SMC. The control law of the MIMO SMC is derived based on an affine transformed model:

$$
\dot{\Psi}=f_{p}(x)+g u
$$

where, the coefficient matrix $g$ is a non-singular square matrix and $f_{p}(x)$ is the part of $f(x, u)$ which does not depend on the inputs. $f_{p}(x)$ and $g$ are found using Eq. (2.28) to 2.32 from the physical model. The sliding surfaces to obtain the control law in the reduced space $\left(Z_{s}\right)$ are defined as:

$$
\begin{gathered}
S=z_{s}-\left(z_{s}\right)_{d} \\
\dot{S}=-\lambda S \\
\dot{z}_{s}=-\lambda\left(z_{s}-\left(z_{s}\right)_{d}\right)+\left(\dot{z}_{s}\right)_{d}
\end{gathered}
$$


where, $z_{s}$ is calculated with respect to SPA nonlinear model order reduction technique, Eq. (2.42), and $\left(z_{s}\right)_{d}$ is the desired reduced order state and is calculated using the desired $\mathrm{AFR}_{d}, \mathrm{~T}_{e x h, d}$, and $\omega_{e, d}$ trajectories. Using Eq. (2.42), the relation between the control law and the state equations in the affine description is:

$$
-\lambda\left(z_{s}-\left(z_{s}\right)_{d}\right)+\left(\dot{z}_{s}\right)_{d}=\widetilde{T}_{p}^{-1} f(x)+\widetilde{T}_{p}^{-1} g u
$$

The final control law is given by:

$$
u=g^{-1} \widetilde{T}_{p}\left[-\lambda\left(z_{s}-\left(z_{s}\right)_{d}\right)+\left(\dot{z}_{s}\right)_{d}-\widetilde{T}_{p}^{-1} f(x)\right]
$$

\subsubsection{HC Emission Control Results}

The reduced SPA SMC is implemented in MATLAB SIMULINK and it is tested against the nonlinear original SMC. The value of the sliding surface gradient is determined such that the desired trajectories will be guaranteed $(\lambda=10)$. Desired AFR and engine speed trajectories are taken from the test results using the ECU of Toyota engine. The exhaust temperature of $650^{\circ} \mathrm{C}$ is chosen for $T_{\text {exh }}$ using available experimental data for the engine [12].

In order to illustrate the advantages of SPA-based technique in tracking desired trajectories, results from reduced-order SPA SMC are compared with those from a full

order SMC (Figure 2.8). As it can be seen, all the three control trajectories are tracked 
with good accuracy; however, desired exhaust gas temperature is tracked faster by the full order SMC compared to the reduced order SMC (Figure 2.8 b). This is because the reduced SPA technique determined that the exhaust gas temperature dynamics have less impact on the tailpipe $\mathrm{HC}$ emission compared to the $\mathrm{HC}$ emission dynamics affected by AFR and idle speed controls in the beginning of the cold start phase. Thus AFR and idle speed controls in the reduced-order SMC are faster than those from the full order SMC (Figure 2.8 a and c). As will be shown later, this will lead to better overall performance of the reduced-order SMC compared to the full order SMC for reducing cumulative tailpipe $\mathrm{HC}$ emission.

(a)

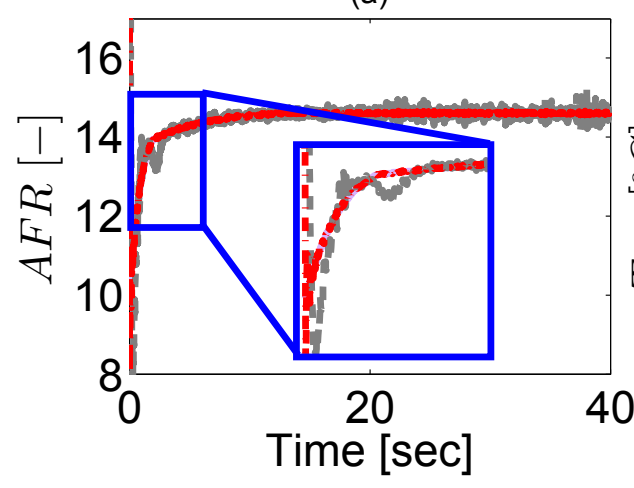

(b)

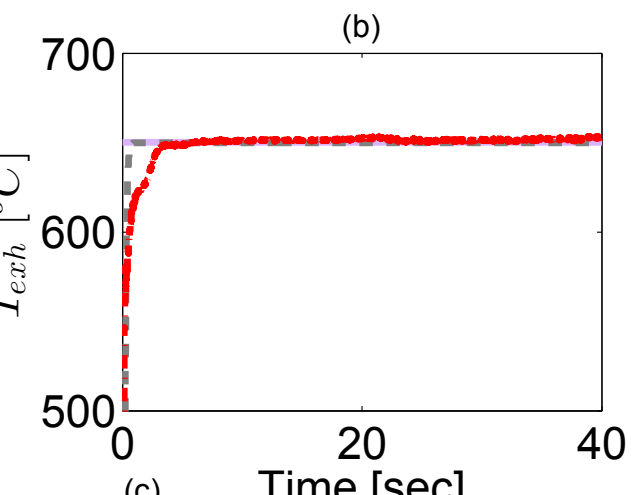

(c) Time $[\mathrm{sec}]$
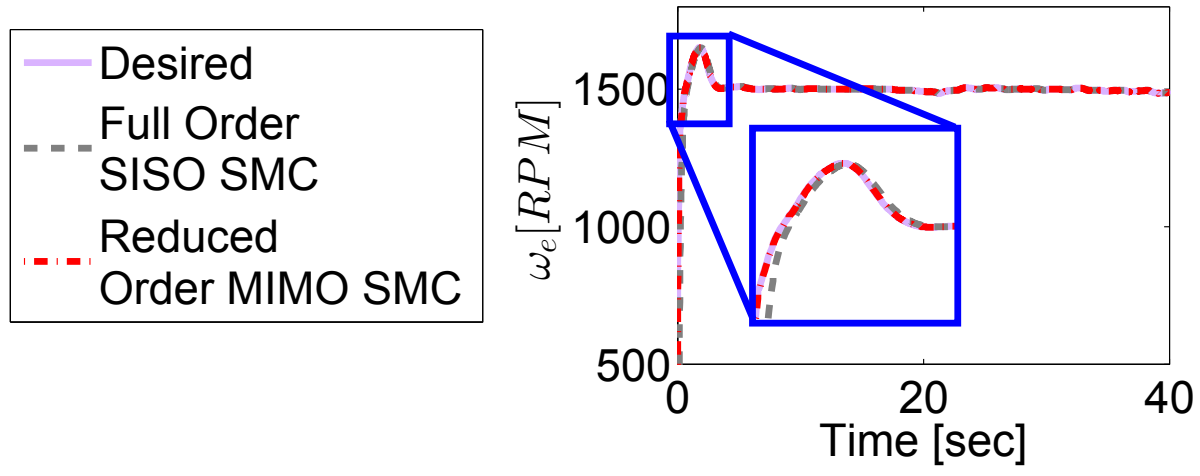

Figure 2.8: Comparison of tracking performance of the designed SMCs: (a) air fuel ratio, (b) exhaust gas temperature, (c) engine speed. 
The main difference among the control inputs from full and reduced order SMCs is spark timing at the very early stage of cold start phase [2]. SPA reduction method puts more emphasis on the parts of system dynamics which have stronger effects on model output. Subsequently, reduced-order SPA SMC tries to adjust control input signals in such a way that tracks the important desired trajectories accurately and reduces the tailpipe $\mathrm{HC}$ emission.

Since SPA puts less emphasis on spark timing control at the very early stage of cold start phase, the reduced-order SMC gives its full authority to AFR and engine speed controls instead. Thus spark timing is not delayed aggressively to meet desired exhaust gas temperature at the very early stage of cold start phase. Delayed ignition causes high engine-out HC emission during cold start. A better adjustment of control inputs in SPA-based SMC leads to a significant reduction in the tailpipe $\mathrm{HC}$ emission as will be discussed by the following figures.

Temperature and conversion efficiency of the catalytic converter are shown in Figure 2.9-a and b. Performances of the both controllers are very similar to each other and no significant advantage is observed by comparing the SMCs. The catalytic converter reaches the light-off in less than 30 seconds. As shown in Figure 2.9 a, the catalytic converter reaches the efficiency of $90.7 \%$ in 40 seconds without using any external source of heating. 

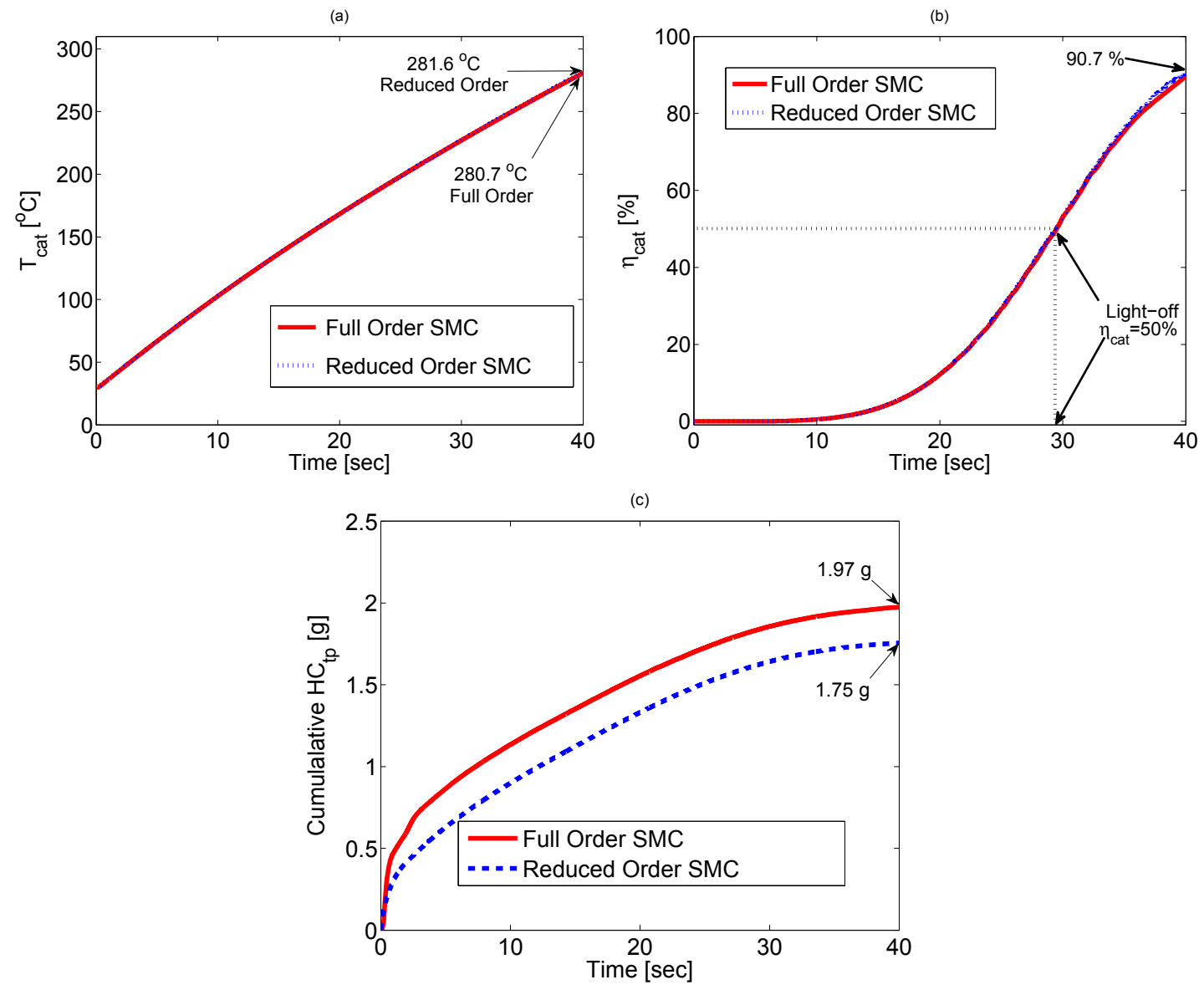

Figure 2.9: Comparison of cold start emission control the full order SMC and the nonlinear reduced-order SPA SMC: (a) catalyst temperature, (b) catalytic converter efficiency, (c) cumulative HC emission.

Finally, the total amount of tailpipe $\mathrm{HC}$ produced during the cold start phase (HC cumulative) from reduced and full order SMCs are shown in Figure 2.9-c. The reducedorder SMC outperforms the full order SMC and produces $12 \%$ less cumulative HC compared to the full order SMC. The final cumulative HC after first 40 seconds in the cold start phase is 1.75 grams which can meet the minimum required HC emission level in the current North America standard for Ultra Law Emission Vehicles (ULEV) [2]. 


\subsubsection{Robustness to Implementation Imprecision}

To evaluate the robustness of the cold start emissions controller, the controller is tested against variations in processor data type sizes, sampling rates and quantization levels. A fixed-point processor is used in this study as it is commonly used for engine control to reduce ECU memory demand. The Toyota Technical Center in North America provided baseline implementation levels for the controller software implementation. Table 2.1 shows the baseline condition characterizing sample specifications for the modeled controller.

Table 2.1

Baseline Condition Used in the Robustness Analysis.

\begin{tabular}{c|c}
\hline \hline Condition & Value \\
\hline ECU update rate & $8 \mathrm{~ms}$ \\
Sampling rate & $8 \mathrm{~ms}$ \\
Quantization level & 12 bit ADC \\
Data type & Fixed point - signed 32 bit \\
Processor type & Embedded micro \\
\hline \hline
\end{tabular}

A compromise between precision and computation load can be decided by evaluating the amount of $H C_{c u m}$. The $H C_{c u m}$ results from the model and controller are shown in Figure 2.10-a as a function of data type in a fixed-point controller. It is found that the fixed-point controller requires at least data with 32-bit precision to meet the required $\mathrm{HC}$ emission limit. The 64-bit fixed point controller produced barely any $H C_{\text {cum }}$ emissions performance benefit over the 32-bit controller; in this case the 32bit controller should be chosen as it provides the best trade-off between computation 
load and precision.

The cold start fixed-point controller is modified to simulate the sampling and quantization imprecision, with each input to the controller sampled then quantized. The results for different sampling rates and signal quantization levels are shown in Figure 2.10 b,c. A dramatic change is observed in $H C_{c u m}$ when the sampling frequency and quantization level are lower than $125 \mathrm{~Hz}$ ( $8 \mathrm{~ms})$ and 12 bit, respectively. This can be due to signal aliasing and distorted input data to the controller. High sampling frequency and quantization levels, however, increase the controller's computation load and memory storage requirement. Similar to the fixed point case, performance differences between $4 \mathrm{~ms}$ and $8 \mathrm{~ms}$ sampling times are not very significant, nor are differences in 16-bit versus 12-bit quantization levels. In each case, the best trade-off occurs in the baseline case.

Results from Section 2.3.4.1 determine the minimum requirements for the controller implementation. If the cold start controller targets on passing the predefined $\mathrm{HC}$ emission limit, specifications of 32-bit fixed point controller with 12-bit quantization level and $125 \mathrm{~Hz}$ sampling frequency are recommended for the ECU implementation process. 
(a) Data type effect
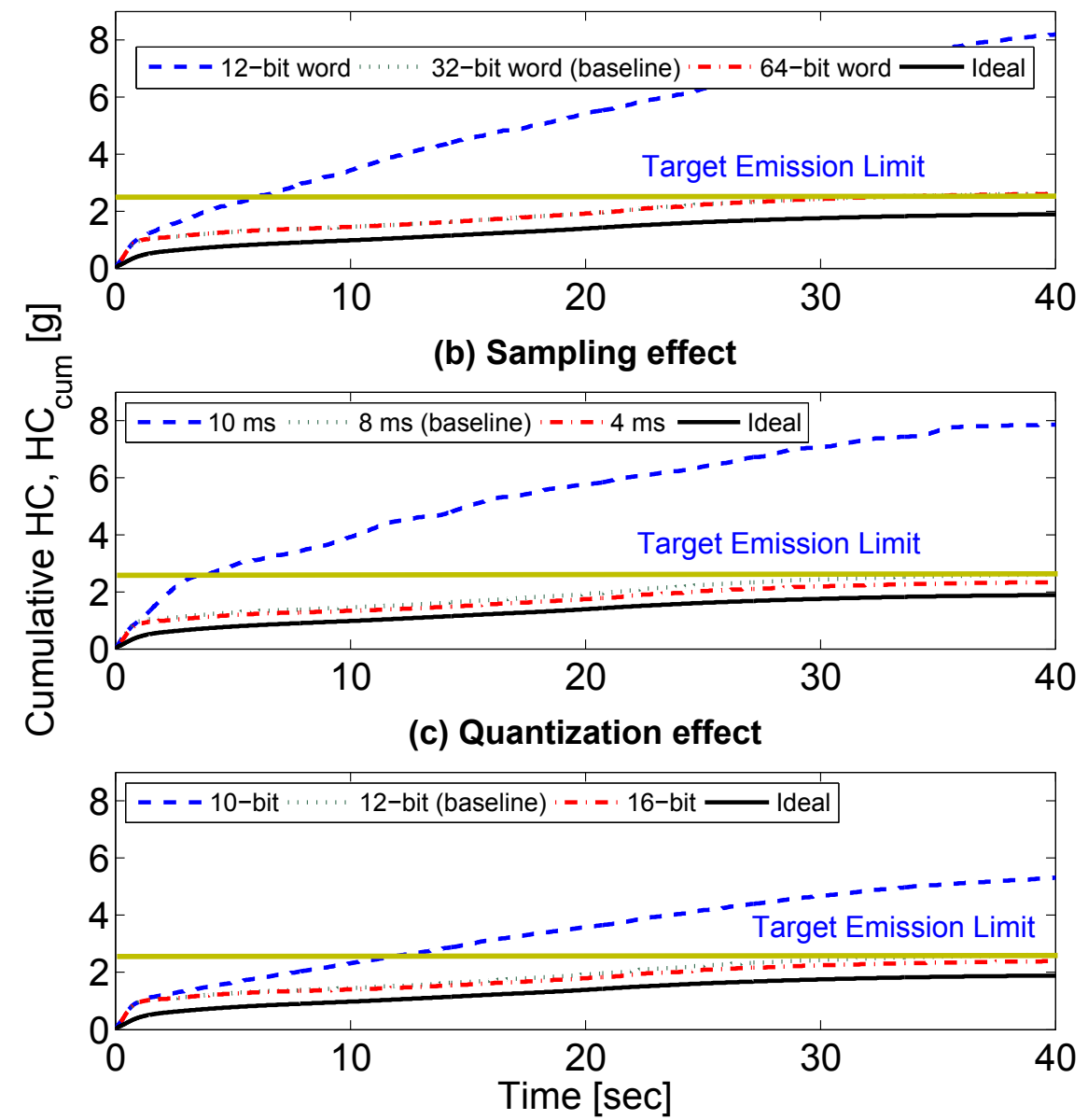

Figure 2.10: Effect of data types, quantization levels and sampling rates on the performance of the cold start controller.

\subsection{Summary and Conclusion}

In this chapter, an early model-based design and verification methodology was developed to identify controller software implementation errors early in the design stage, to reduce the debugging efforts in a controller's V\&V cycle and also to improve a controller's robustness against implementation imprecision. In the first step, a technique 
of model order reduction was developed to simplify the model for controller design, thus decreasing the controller's calibration efforts and decreasing required V\&V time. In particular, a novel nonlinear technique based on balanced realization and singular perturbation approximation was developed to reduce the order of a nonlinear model. The second step included design of a reduced order model-based controller using the reduced order plant model. The results of the reduced order SMC were compared with those from a full order SMC. The simulation results showed that the designed reduced order SMC outperforms the full order SMC in terms of engine-out and tailpipe $\mathrm{HC}$ emission with about $12 \%$ reduction in $\mathrm{HC}$ tailpipe emission. In the third step, an early MIL platform was developed to test the controller robustness to three main implementation imprecisions, including fixed-precision arithmetic, quantization level, and sampling rate. The proposed methodology was demonstrated on a reduced order MIMO SMC automotive controller which was designed to decrease the cold start HC emissions in a passenger car. The methodology was evaluated on an experimentally validated cold start model. 


\section{Chapter 3}

\section{Easily Verifiable Continuous-Time Controller Design ${ }^{1}$}

\subsection{Introduction}

A model-based uncertainty-adaptive methodology is presented in this chapter to enable easily verifiable controller design based on the formulation of a continuous-time sliding mode controller (SMC). The proposed adaptive SMC improves the controller robustness against sampling and quantization imprecisions. An effective approach to overcome imprecisions during controller implementation is early model-based design and verification of the controller, in which, the controller structure is investigated and modified to improve the robustness against arising implementation imprecisions [1]. Three early verification approaches have been proposed in the literature, each of which can significantly improve the controller robustness against implementation imprecisions. The first approach is to investigate the controller structure and find the

\footnotetext{
${ }^{1}$ This chapter has been published in the proceeding of 2016 SAE World Congress [3] (doi:10.4271/2016-01-0629). Reprinted with permission by SAE @ 2017 SAE International as shown in Appendix D. Further distribution of this material is not permitted without prior permission from SAE.
} 
sources of numerical noise propagation and remove them [1]. The second approach is to detect the imprecisions during the early stages of the controller design, predicting the maximum uncertainty bounds on measured signals due to ADC and re-design the controller to implement the propagated uncertainty bounds on the state equations into the controller architecture to improve the robustness [23]. Finally, the third approach is to develop discrete control theory to transfer the conventional controller from continuous time to discrete space and modify the control commands by inclusion of uncertainty bounds on control signals [24]. The second approach will be investigated in this chapter, while the third approach will be studied in Chapter 4.

Incorporation of the implementation imprecisions knowledge into a SMC design leads to improvement in the robustness characteristics of the implemented controller [23, 24]. In this approach, the controller is re-designed to incorporate the maximum propagated uncertainty (sampling and quantization) bounds on state equations [23] and control signals [24]. Although the results in [23] and [24] showed that the modified controllers are able to improve the controller performance under implementation imprecisions, incorporation of the maximum uncertainty bounds, based on worst case scenarios, makes the controller too conservative and leads to large control actions. Besides, calculating overall uncertainty bounds requires an ideal symbolic model of the controller, which means it cannot provide an online framework to calculate uncertainty bounds, since it is too time consuming. 
The main contribution of this chapter is to develop a generic theoretical framework to design easily verifiable controller, based on SMC formulation, that is robust to ADC implementation imprecisions, in which, not only the controller is updated online, but also the conservative controller design will be avoided. SMC is selected as the baseline control framework in this study, because it is one of the few controller designs that can treat systems with very nonlinear dynamics with a great deal of uncertainty [1, 57]. The proposed SMC in this chapter has a variable structure in which the control actions are updated according to an embedded adaptation algorithm to reduce the tracking errors under implementation imprecisions.

\subsection{Uncertainty Adaptive SMC Robust to Imple- mentation Imprecisions}

Any nonlinear dynamic system can be expressed in the following general form:

$$
\dot{x}=f(x, u)
$$

where $x \subset X \in \mathbb{R}^{n}$, and $u \subset U \in \mathbb{R}^{h}$ are the state, and the input vectors, respectively. It can be assumed that each state of the system in Eq. (3.1) can be controlled by one physical or synthetic control input $(n=h)$. In practice, since the measured signals from a plant are sampled and quantized and also control commands are updated with a rate equal to the sampling rate, implementation imprecisions should be included in the state equations. If the uncertainties due to implementation are assumed to be 
bounded and also considered as the dominant source of uncertainties, the nonlinear system under implementation imprecisions can be re-written as follows [87]:

$$
\dot{x}=f(x, u)+\Delta f(x, u), \quad\|\Delta f(x, u)\| \leq \beta
$$

where $\Delta f(x, u)$ represents the uncertainty term and is caused by implementation imprecisions. The uncertainty is assumed to be bounded $(\|\Delta f(x, u)\| \leq \beta)$. The sliding surface $(s)$ and reaching law are defined as follows to converge the states of the system towards the sliding surface $\left(x \rightarrow x_{d} \Rightarrow s \rightarrow 0\right)$, and also compensate for the introduced implementation imprecisions [57]:

$$
s=x-x_{d} \Rightarrow \dot{s}=\dot{x}-\dot{x_{d}}=-\lambda s-K \times s a t\left(\frac{s}{\phi}\right)
$$

where $\phi$ is a tunable design parameter that represents the boundary layer thickness of the sliding surface and $\lambda$ is a gradient of the sliding function. $K$ is assumed to be able to compensate for implementation imprecisions. $K$ can be either a fixed gain or a variable parameter calculated using an adaptation algorithm, as will be discussed in the following.

The objective of this study is to develop an online technique to overcome implementation imprecisions and avoid conservative controller design which is caused if a fixed gain based on maximum uncertainty bounds [23] is applied. Here, an adaptive approach is proposed to calculate $K$ for minimizing the tracking error to account for implementation uncertainties. 
$K$ in Eq. (3.3) is calculated by solving the following adaptation law:

$$
\dot{K}=-\gamma|s| \times \operatorname{sat}\left(\frac{s}{\phi}\right)
$$

where $\gamma$ is a positive constant adaptation gain. Lyapunov's direct method [57] is employed to guarantee the SMC stability with the adaptation law in Eq. (3.4). It can be observed from Eq. (3.4) that the solution of this first order differential equation is always bounded, if the sliding surface $(s)$ converges to zero. Since the sliding surface convergence and adaptation mechanism occur simultaneously, the Lyapanuv function should reflect the zero-convergence of both $s$ and $K$. To this end, the following candidate Lyapunov function $(V)$ is chosen:

$$
V=\frac{1}{2} s^{2}+\frac{1}{2} K^{2}
$$

where the following conditions for the Lyapunov function should be met:

$$
\begin{cases}V(s, t)>0 & \text { if } s \neq 0 \\ V(0, t)=0+\frac{1}{2} K^{2}=0 & \text { if } s=0\end{cases}
$$

The first condition is always satisfied, while the second condition dictates that $K(0, t)=0$. This condition is used to confirm that $V$ in Eq. (3.5) is always positive definite, if the adaptation law in Eq. (3.4) is solved with respect to $K(0, t)=0$ as the boundary layer condition. Derivative of $V$ is calculated as follows using Eq. (3.3) 
and (3.4):

$$
\begin{gathered}
\dot{V}=s \dot{s}+K \dot{K} \\
=s\left\{-\lambda s-K \times \operatorname{sat}\left(\frac{s}{\phi}\right)\right\}+K \dot{K} \\
=-\left\{\lambda s^{2}+K\left(s \times \operatorname{sat}\left(\frac{s}{\phi}\right)\right)\right\}+K \dot{K} \stackrel{\dot{K}=-\gamma|s| \times \operatorname{sat}\left(\frac{s}{\phi}\right)}{\longrightarrow} \\
=-\left\{\lambda s^{2}+K\left(1+\gamma \frac{|s|}{s}\right) s \times \operatorname{sat}\left(\frac{s}{\phi}\right)\right\}
\end{gathered}
$$

$\dot{V}$ depends on the sign of the sliding function, therefore it can be divided into the following equation:

$$
\dot{V}=\left\{\begin{array}{l}
-\lambda s^{2}-K(1+\gamma) s \times \operatorname{sat}\left(\frac{s}{\phi}\right) \quad \text { if } \quad s>0 \\
-\lambda s^{2}-K(1-\gamma) s \times \operatorname{sat}\left(\frac{s}{\phi}\right) \quad \text { if } \quad s<0
\end{array}\right.
$$

where $s \times \operatorname{sat}\left(\frac{s}{\phi}\right) \geqslant 0$ and $K>0$. Since $\gamma$ is assumed to be a positive constant, $\dot{V}$ is negative definite if, and only if $1>\gamma>0$. In other words:

$$
\stackrel{\text { if } 1>\gamma>0}{\longrightarrow}\left\{\begin{array}{l}
\dot{V}(s \neq 0, t)<0 \\
\dot{V}(s=0, t)=0
\end{array}\right.
$$

Since the Lyapunov function $V$ is positive definite and its derivative $\dot{V}$ is negative definite, the controller is asymptotically stable [57]. Figure 3.1] shows the schematic of the closed-loop system with the proposed adaptive SMC. Implementation imprecisions are introduced by an ADC unit which does the sampling and quantization on measured signals. The adaptation mechanism in Figure 3.1 updates the gain $K$ at each sampling period online according to the measured $(X)$ and desired values 
$\left(X_{d}\right)$ for each state of the plant. In the next section, application of the uncertainty adaptive SMC is shown for a highly nonlinear engine control problem.

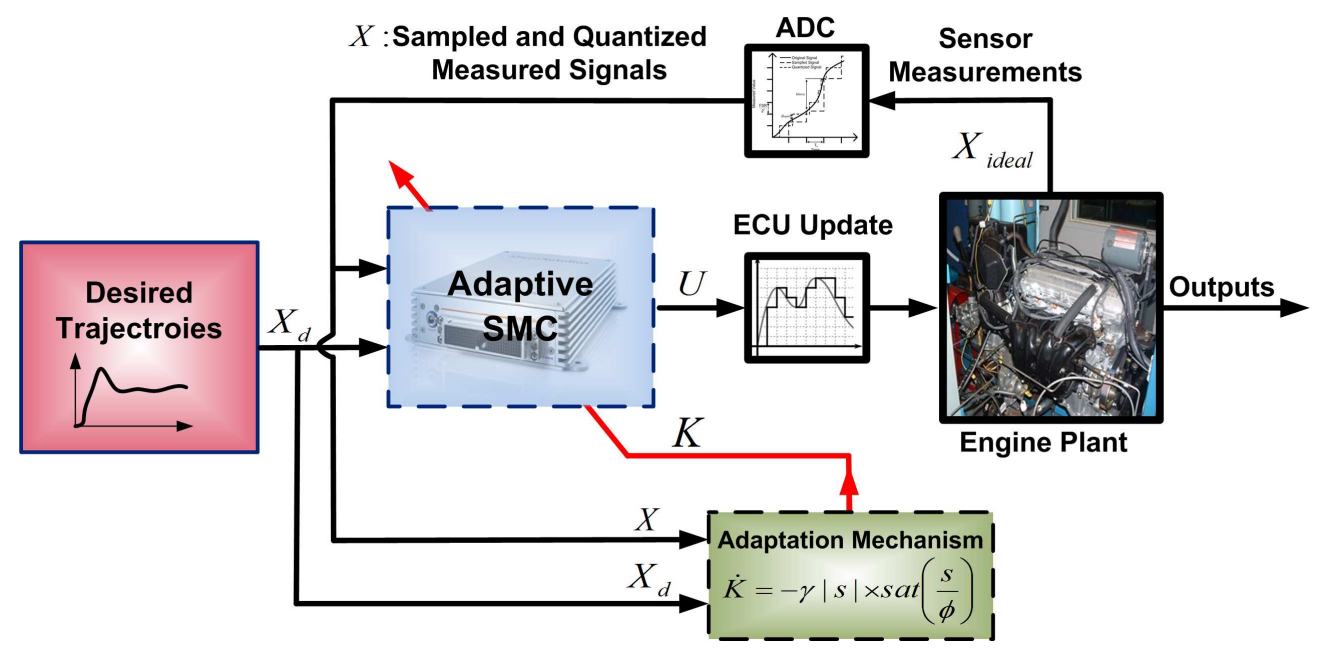

Figure 3.1: Proposed adaptive SISO SMC to account for implementation imprecisions.

\subsection{Case Study: Cold Start Emission Control}

The SI engine cold start emission model in Chapter 2 with Eq. (2.28) to $(2.32)$ is used in this section to apply the proposed adaptive SMC under ADC imprecisions of sampling time $=10 \mathrm{~ms}$ and quantization level=10 bit. The goal is to keep exhaust temperature, engine speed, and air to fuel ratio (AFR) at desired values. For better controller evaluation, desired trajectories which should be tracked by the implemented SMC are defined in their worst and non-smooth shapes. 


\subsubsection{Baseline SISO Sliding Controller Design \& Impact of Implementation Imprecisions}

The engine cold start model with Eq. (2.28)-2.32) is used to study the impacts of implementation imprecisions (sampling time $=10 \mathrm{~ms}$ and quantization level=10 bit) on the baseline SMC performance. The reason for studying the controller performance under $10 \mathrm{~ms}$ of sampling time and quantization level of 10 bit is that these values were recommended as the "minimally implemented" controller in [1, 12]. The main control target, similar to Chapter 2 is reducing $H C_{c u m}$, which can be achieved by keeping exhaust temperature, engine speed, and AFR at desired values. For better controller evaluation, desired trajectories which should be tracked by the implemented SMC are first defined in their worst and non-smooth shapes. After validation of the proposed adaptive controller with non-smooth trajectories, the final SMC will be tested against the desired trajectories of a real ECU to demonstrate the benefit of using the new controller in reducing $H C_{\text {cum }}$.

Higher exhaust gas temperature is required for fast catalytic converter light-off. Exhaust temperature is controlled by spark timing and higher exhaust temperature is achieved by delayed spark timing. However, delayed spark timing leads to high engine-out HC emission. Thus, there is a trade-off between fast catalytic converter light-off and low engine-out $\mathrm{HC}$ emission. The first sliding surface of the controller is defined to be the error in tracking the desired exhaust temperature trajectory. Since AFR is a function of air and fuel mass flow rates and air mass flow controls the 
engine speed, AFR cannot be a good choice as a sliding surface. Thus, the second sliding surface is defined to be the error in tracking the desired fuel mass flow which is estimated with respect to the desired AFR trajectory.

Engine speed is considered as the third sliding surface. The rate of engine speed impacts the exhaust gas flow rate for heating the catalytic converter. There is no explicit control input for tracking the desired engine speed, therefore, desired air mass is considered as the synthetic control input for the engine speed tracking problem. Introducing the desired air mass requires defining another sliding surface to track the desired air mass that is calculated from desired AFR trajectory. Overall, four sliding surfaces are defined as follows:

$$
\begin{gathered}
s_{1}=T_{e x h}-T_{e x h, d}, \quad s_{2}=\dot{m}_{f}-\dot{m}_{f, d} \\
s_{3}=\omega_{e}-\omega_{e, d}, \quad s_{4}=m_{a}-m_{a, d}
\end{gathered}
$$

$T_{e x h}, A F R\left(\dot{m}_{f}\right), m_{a}$, and $\omega_{e}$ can be measured on an engine; thus the ADC affects these four measured states and corresponding sliding surfaces. A baseline SISO SMC with four control inputs can be designed to drive the states of the system $(x)$ towards the desired values $\left(x_{d}\right)$ using the following sliding surface and reaching law [57]:

$$
s=x-x_{d} \Rightarrow \dot{s}=\dot{x}-\dot{x_{d}}=-\lambda s
$$


Control inputs for the baseline SISO SMC are calculated according to Eq. 2.28)(2.32), Eq. (3.10), and (3.11):

$$
\begin{gathered}
\Delta=\frac{1}{7.5}\left(\frac{\tau_{e}}{A F I}\left[\frac{T_{e x h}}{\tau_{e}}+\dot{T}_{e x h, d}-\lambda_{1} s_{1}\right]-600\right) \\
\dot{m}_{f c}=\dot{m}_{f}+\tau_{f}\left[\frac{d}{d t} \dot{m}_{f, d}-\lambda_{2} s_{2}\right] \\
\dot{m}_{a i}=-\lambda_{3} s_{3}+\dot{m}_{a o}+\dot{m}_{a, d} \\
m_{a, d}=\frac{1}{30000}\left[J\left(-\lambda_{4} s_{4}+\omega_{e, d}\right)+0.4 \omega_{e}+100\right]
\end{gathered}
$$

where, $\lambda_{1,3,4}=10$ and $\lambda_{2}=100$. Results of desired trajectories tracking from the baseline SISO SMC under ADC imprecisions are compared in Figure 3.2 with those from an ideal SMC. The ideal SMC has the same structure of the baseline SMC, but no ADC imprecisions have been introduced on the controller input/output (I/O). As it can be seen, the tracking performance of the controller degrades upon the introduction of ADC imprecisions on feedback signals from the plant and also control inputs.

AFR tracking in Figure $3.2 \mathrm{r}$ a is affected the most by ADC imprecisions, to the point that the tracking behavior of AFR controller is not acceptable (AFR tracking error increases over 10 times compared to the ideal controller). Engine speed controller is not able to track the desired profile under implementation impressions when a sharp change occurs in the desired engine speed. When the desired $T_{\text {exh }}$ changes rapidly, exhaust temperature controller fails to respond fast under ADC imprecisions. 
(a)

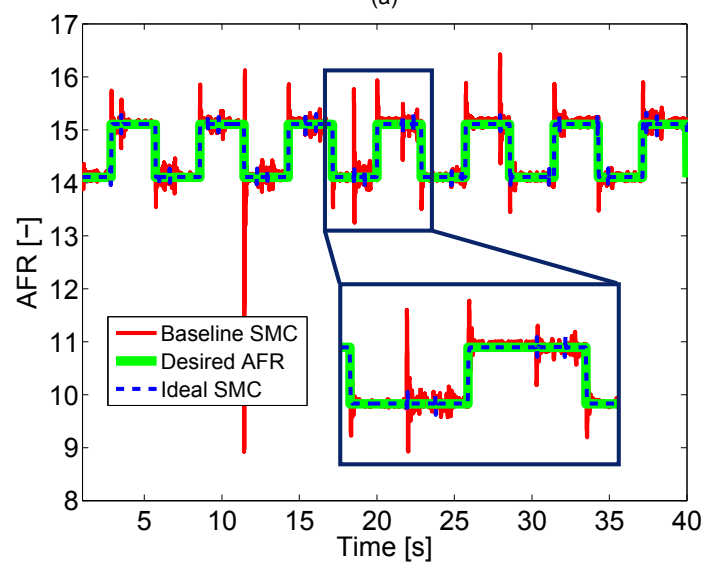

(b)

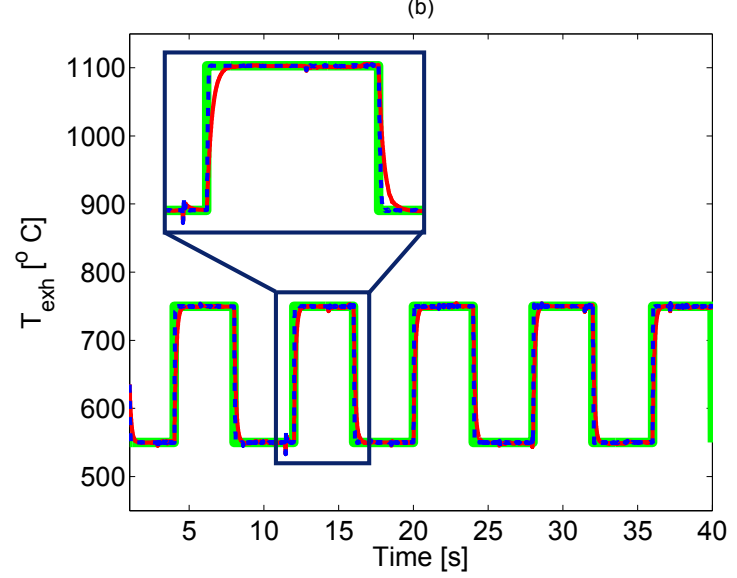

(c)

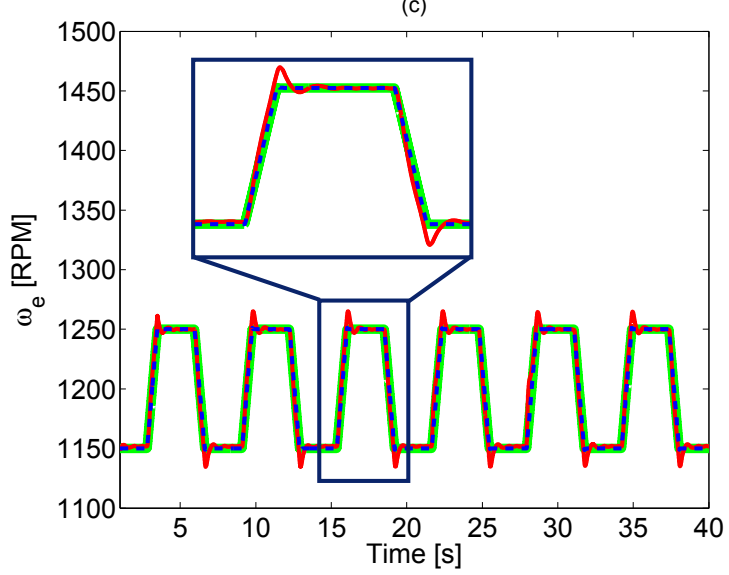

Figure 3.2: $\quad$ Effects of ADC imprecisions (sampling time = $10 \mathrm{~ms}, E C U$ update period $=10 \mathrm{~ms}$, and quantization level $=10 \mathrm{bit}$ ) on baseline SMC for tracking (a) AFR, (b) exhaust gas temperature $\left(T_{e x h}\right)$, and $(\mathrm{c})$ engine speed $\left(\omega_{e}\right)$.

Overall, if the cold start controller aims to meet the target $\mathrm{HC}$ emission limit, the implementation of a baseline SMC in a real ECU, with ADC imprecisions, cannot guarantee the desirable performance of the engine. The next section investigates the performance of the proposed uncertainty adaptive SMC to improve the robustness of the baseline controller against implementation imprecisions. 


\subsubsection{Uncertainty-Adaptive SISO Sliding Controller Design}

The baseline SMC in the previous section is modified by incorporating the adaptation mechanisms for each of the sliding surfaces in Eq. 3.10). Control inputs for the adaptive SISO SMC are calculated according to Eq. 3.3$),(2.28)-(2.32)$, and $(3.10)$ :

$$
\begin{gathered}
\Delta=\frac{1}{7.5}\left(\frac { \tau _ { e } } { A F I } \left[\frac{T_{e x h}}{\tau_{e}}+\dot{T}_{e x h, d}-\lambda_{1} s_{1}\right.\right. \\
\left.\left.-K_{1} s a t\left(\frac{s_{1}}{\phi_{1}}\right)\right]-600\right) \\
\dot{m}_{f c}=\dot{m}_{f}+\tau_{f}\left[\frac{d}{d t} \dot{m}_{f, d}-\lambda_{2} s_{2}-K_{2} s a t\left(\frac{s_{2}}{\phi_{2}}\right)\right] \\
\dot{m}_{a i}=-\lambda_{3} s_{3}-K_{3} s a t\left(\frac{s_{3}}{\phi_{3}}\right)+\dot{m}_{a o}+\dot{m}_{a, d} \\
m_{a, d}=\frac{1}{30000}\left[J\left(-\lambda_{4} s_{4}-K_{4} s a t\left(\frac{s_{4}}{\phi_{4}}\right)+\omega_{e, d}\right)+0.4 \omega_{e}+100\right]
\end{gathered}
$$

where $K_{1,2,3,4}$ are updated according to Eq. (3.4) for each sliding surface. Values of $K_{1,2,3,4}$ are plotted in Figure 3.3 , where the maximum uncertainty bounds on state equations of the engine model are adopted from [23]. The tuning parameters $(\lambda, \phi$, and $\gamma)$ of the SMCs are reported in the Appendix A. Table 3.1 shows the performance of three adaptive SMCs under ADC implementation imprecisions for tracking the same desired trajectories shown in Figure 3.2. The calculated gains of the adaptive SMC not only lie between upper and lower uncertainty bounds for all state equations, but also the values are calculated online at each time step, so they are adaptive to the engine varying conditions. 
Table 3.1

Mean value of the tracking errors $\left(\frac{\int|e| d t}{\Delta t}\right)$ from the baseline and adaptive SISO SMCs.

\begin{tabular}{c|c|c} 
Controller & $\begin{array}{c}\text { Baseline SMC } \\
\text { Reference }\end{array}$ & $\begin{array}{c}\text { Adaptive SMC } \\
\text { SISO }\end{array}$ \\
\hline \hline AFR $[-]$ & 0.146 & $\begin{array}{c}0.143 \\
(-2.05 \% \downarrow)^{\dagger}\end{array}$ \\
\hline$T_{\text {exh }}\left[^{\circ} C\right]$ & 10 & $\begin{array}{c}5.5 \\
(-45.0 \% \downarrow)\end{array}$ \\
\hline $\begin{array}{c}\text { Engine } \\
\text { Speed, } \omega_{e}[R P M]\end{array}$ & 8.5 & $\begin{array}{c}7.1 \\
(-16.5 \% \downarrow)\end{array}$ \\
\hline \hline Fuel & 28.010 & $\begin{array}{c}28.005 \\
(-0.02 \% \downarrow)\end{array}$ \\
\hline Consumption $[\mathrm{g}]$ & &
\end{tabular}

$\dagger$ values inside the parentheses show the improvements by reduction in tracking errors using the adaptive SMC compared to the baseline SMC.
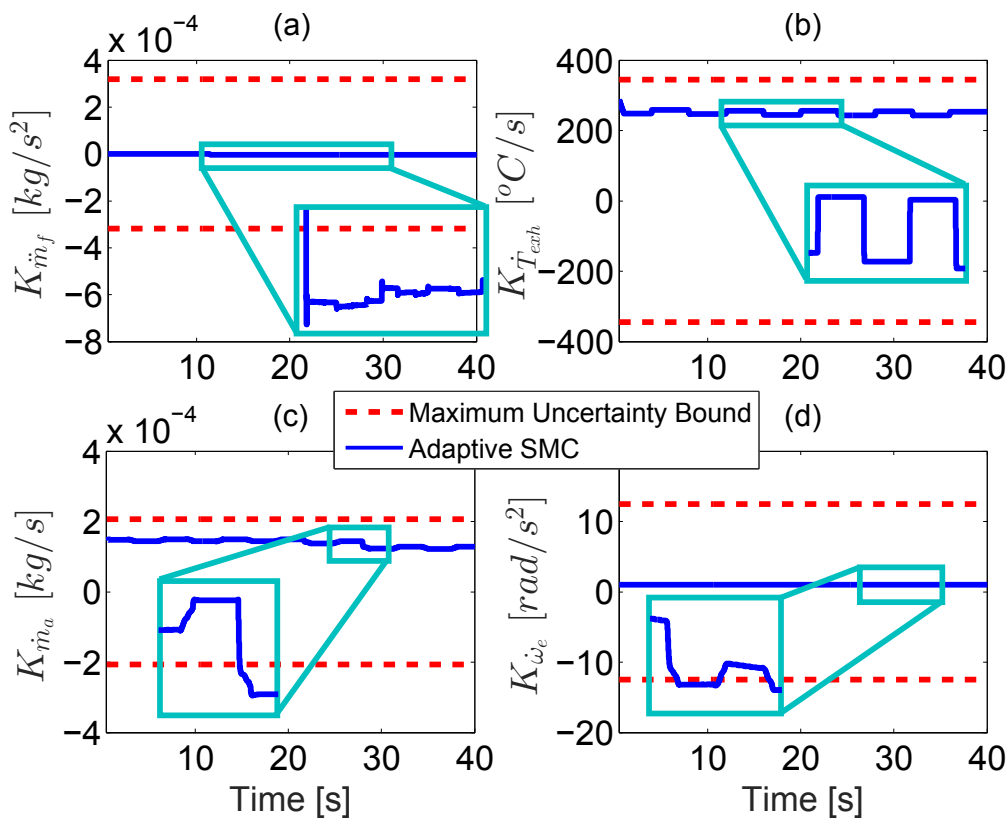

Figure 3.3: Verification results of the uncertainty gains from the SISO adaptive SMC for (a) fuel flow, (b) exhaust temperature, (c) intake air flow, and $(d)$ engine speed state equations. 
The exhaust temperature tracking is improved by $45 \%$ using the adaptive SMC compared to the baseline SMC (Table 3.1). The same pattern is found for the engine speed tracking, showing that the performance of the adaptive SMC (16.5\% improvement) is better than the baseline SMC. Finally, there is also $2 \%$ improvement in the desired AFR tracking using the adaptive SMC. In addition, Table 3.1 shows that despite the better performance from the adaptive SMC, there is no significant change in the total fuel consumption during the first $40 \mathrm{sec}$ of the engine operation compared to the baseline SMC.

Unlike exhaust gas temperature which is dominantly affected by spark timing significantly, the relationships among other three state equations (air mass, fuel flow rate, and engine speed) are more complex that make it hard to decouple sliding surfaces from each other, and tune/control each SISO SMC independently. AFR and engine speed are both functions of the air mass. Putting more efforts to track one of them more precisely leads to failure in tracking the other desired trajectory.

Although the SISO SMC design is simple and straightforward, because of the interaction among the sliding surfaces due to nonlinear engine dynamics, the optimum trade-off for tuning different SMCs is hard to achieve. Figure 3.4 explains that when engine speed changes rapidly, due to the strong correlation among sliding surfaces, errors in AFR tracking increase considerably. In the next section, a MIMO adaptive 
SMC is formulated to improve the controller robustness against implementation imprecisions using the proposed adaptive framework. The MIMO controller prioritizes the states of the engine model based on their energy and put more efforts to track trajectories with higher level of energy. In addition, the MIMO controller can be tuned using fewer parameters, compared to the SISO controller.

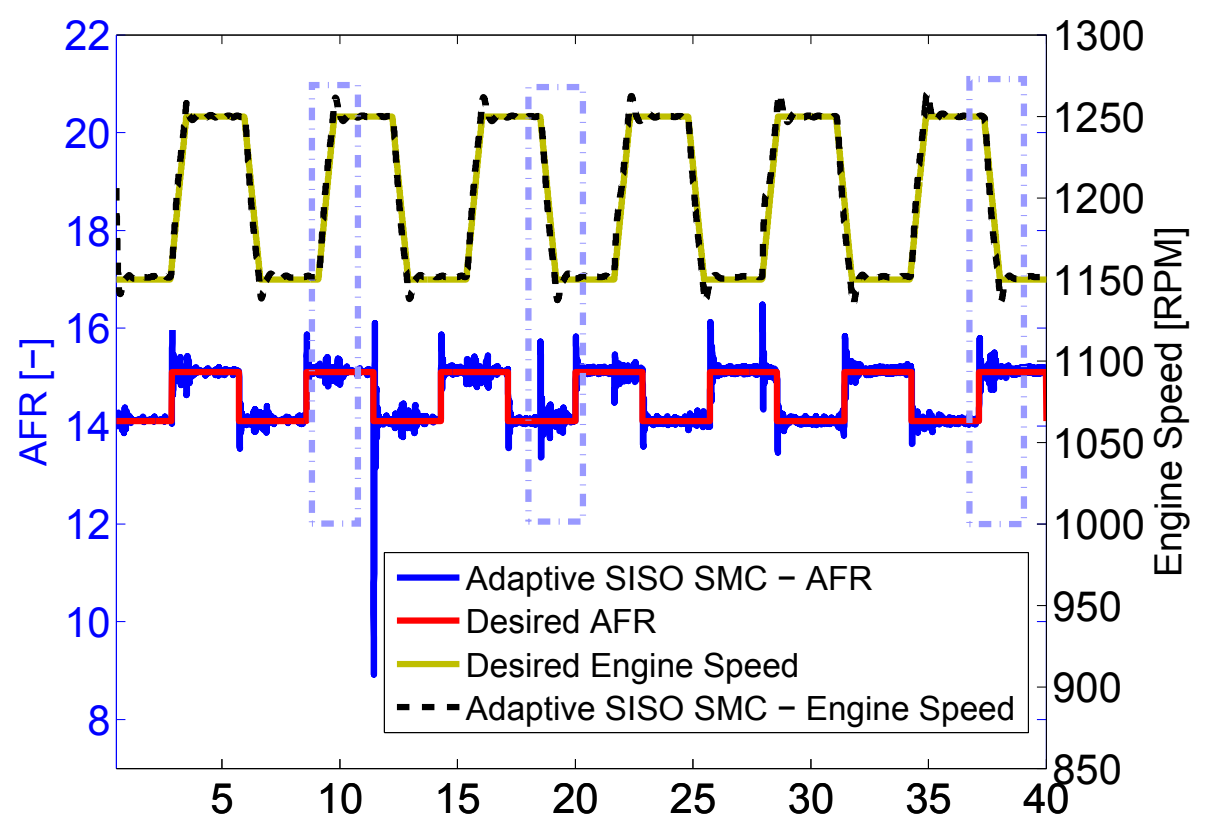

Figure 3.4: Impact of AFR and engine speed control on each other from SISO SMC.

\subsubsection{MIMO Uncertainty-Adaptive SMC Design}

Tuning of the cold start SISO controller in the previous section is a cumbersome process due to the involved coupling in the plant dynamics. One effective approach to reduce the destructive interaction among sliding surfaces on the adaptive controller 
performance is to design the controller in a MIMO structure using a balanced realization form of the engine cold start model [2]. The state-space after balanced realization is a non-physical space $(Z)$ in which the controllability and observability gramians are diagonal and equal to the Hankel singular value (HSV) matrix [64].

The transformation from the physical state space $(X)$ into the non-physical state space $(Z)$ is conducted using the nonlinear balanced realization [1, 55], which was discussed in details in Chapter 2, When the SMC is designed with respect to states in $Z$ space, the problem of finding optimum trade-off among control inputs will be addressed inherently. This is because the states in $Z$ space are prioritized based on their energy levels. The designed SMC in $Z$ space, as will be shown later, needs fewer tuning parameters, while puts an optimized weight among the three control actuations, since it evaluates the importance of affecting dynamics through nonlinear balanced realization.

The balanced state-space realization is calculated using the $x=T \times z$ transformation. $T$ is defined as the coordinate transition matrix which transfers the space of the physical model $(X)$ to the non-physical space $(Z)$ where the system is balanced. Similar to Chapter 2, we define a state transition matrix, $T_{p}$, which is part of $T$ in which the column associated with $T_{\text {cat }}$ is taken away. The control law of the MIMO SMC is derived based on an affine transformed model and recalling Eq. (2.44) and 2.45). The 
vector of sliding surfaces $(S)$ to obtain the control law in $Z$ space are defined as:

$$
\begin{gathered}
S=z-z_{d} \\
\dot{S}=-\lambda S-\kappa \times \Upsilon\left(\frac{S}{\phi}\right) \\
\dot{z}=-\lambda\left(z-z_{d}\right)+\dot{z}_{d}-\kappa \times \Upsilon\left(\frac{S}{\phi}\right)
\end{gathered}
$$

where, $z=T_{p}^{-1} \Psi$, and $z_{d}$ is the desired state vector in $Z$ space. $\Upsilon\left(\frac{S}{\phi}\right)$ and $\kappa$ are as follows:

$$
\begin{gathered}
\Upsilon\left(\frac{S}{\phi}\right)=\left[\operatorname{sat}\left(\frac{s_{1}}{\phi}\right), \ldots, \text { sat }\left(\frac{s_{4}}{\phi}\right)\right]^{\top} \\
\kappa=\operatorname{diag}\left[K_{1}, K_{2}, K_{3}, K_{4}\right]
\end{gathered}
$$

where $K_{1, . .4}$ are updated according to Eq. (3.4). Sliding surface boundary layer $(\phi)$ and adaptation gain $(\gamma)$ are the same for all sliding surfaces, since all physical states are normalized prior to be transferred to $Z$ space. The relation between the control law and the state equations in the affine description is:

$$
-\lambda\left(z-z_{d}\right)+\dot{z}_{d}-\kappa \times \Upsilon\left(\frac{S}{\phi}\right)=T_{p}^{-1} f(\Psi)+T_{p}^{-1} g u
$$

The final control law is given by:

$$
u=T_{p} g^{-1}\left[-\lambda\left(z-z_{d}\right)+\dot{z}_{d}-\kappa \times \Upsilon\left(\frac{S}{\phi}\right)-T_{p}{ }^{-1} f(\Psi)\right]
$$

Figure 3.5 shows the structure of the proposed MIMO adaptive SMC for the SI engine during cold start phase under ADC imprecisions. 


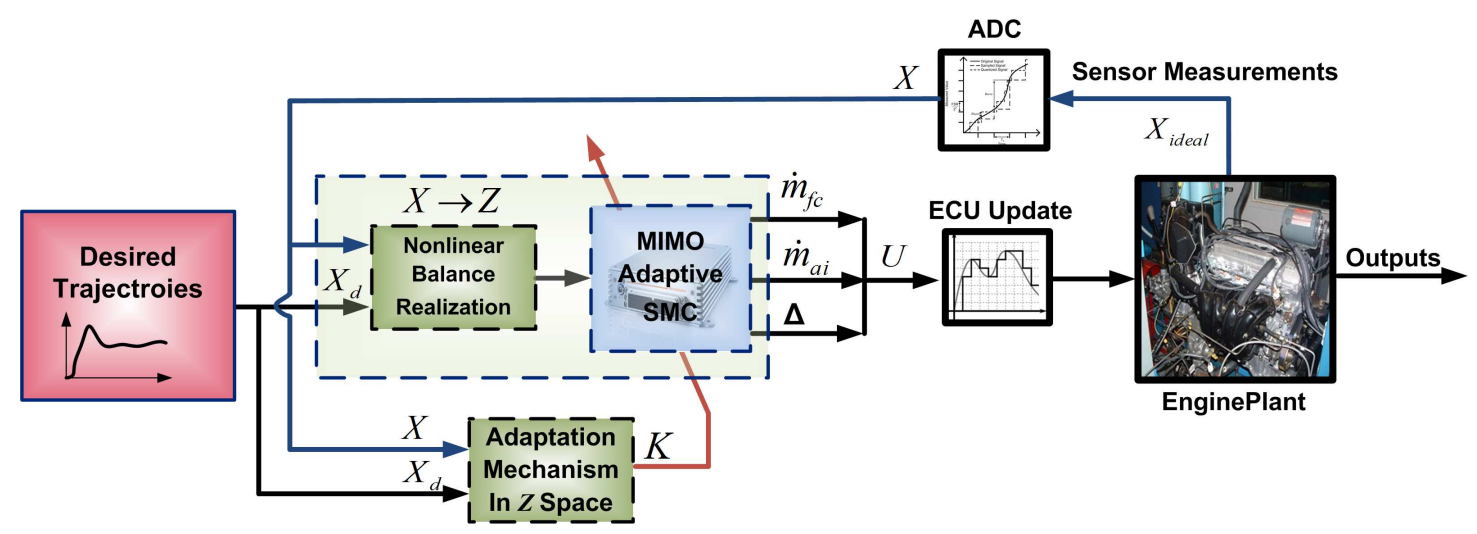

Figure 3.5: Schematic of the adaptive MIMO SMC under implementation imprecisions. The MIMO SMC incorporates a plant model derived by applying nonlinear balanced realization.

\subsubsection{Real-Time Controller Verification}

A processor-in-the-loop (PIL) platform is designed to verify the performance of the designed controllers for real-time operation. This PIL setup will be used in the next chapters for real-time controller verification purpose. Figure 3.6 shows the designed PIL platform in which, dSPACE MicroAutoboxII (MABX) is employed as a controller and National Instrument (NI) PXI processor (NI PXIe-8135) is employed to represent the engine plant in virtual (i.e., model) environment. Engine performance and tracking of desired trajectories are monitored real-time using the MABX and the PXI under embedded ADC imprecisions on the plant feedback signals from NI PXI processor. MABX is a rapid control prototype (RCP) system and operates like a real ECU. dSPACE Control Desk ${ }^{\circledR}$ software on the desktop computer is used for 


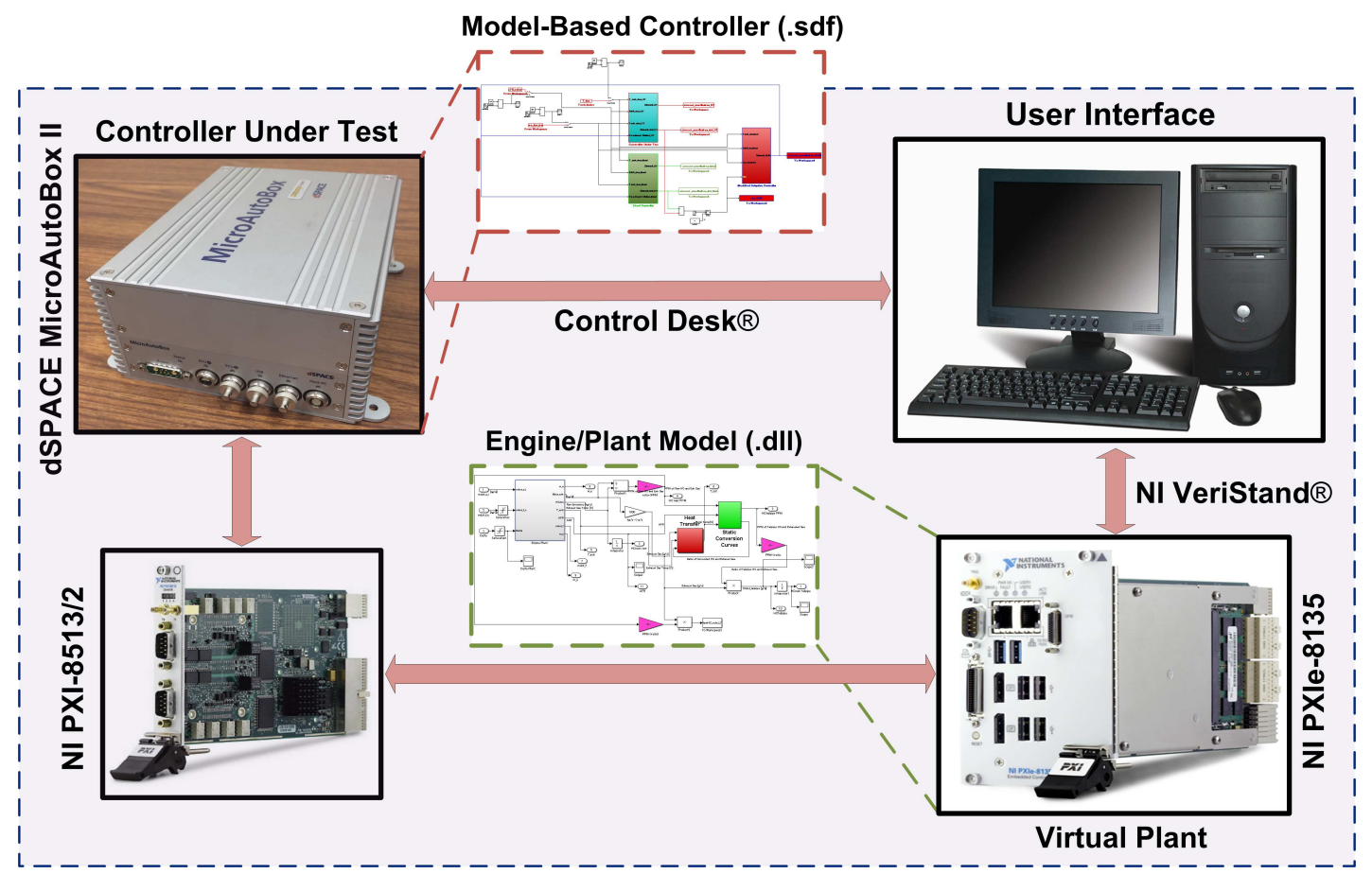

Figure 3.6: Schematic of the processor-in-the-loop (PIL) setup for real-time controller verification.

controlling the hardware real-time. NI PXI provides a flexible platform for measurement and control. Real-time test configuration for NI PXI is conducted using NI VeriStand ${ }^{\circledR}$ software on the interface desktop computer. Access to NI PXI possessor input/output (I/O) and interfaces (NI PXI-8135/2) is granted by VeriStand.

The proposed adaptive MIMO SMC and engine model are divided into two MATLAB/Simulink ${ }^{\circledR}$ files $(. m d l)$. The MIMO adaptive SMC .mdl file is first updated with dSPACE RTI block sets on I/Os. Then, the file is converted to C-code using DS1401 compiler. The generated C-code of the controller (.sdf) is implemented into the MABX using Control Desk. Similarly, the engine model is compiled using 
NI VeriStand compiler and generated C-code file (.dll) is built into the PXI. Sampler and quantizer blocks are embedded on engine model I/Os to virtually emulate the ADC effects on the controller performance. The implemented adaptive MIMO SMC in the MABX communicates with the embedded engine model inside the PXI processor through NI PXI-8513/2 interface.

Table 3.2 shows the performance of the applied MIMO adaptive SMC on the engine model under $10 \mathrm{~ms}$ of sampling and 10 bit of quantization level. The comparison between the tracking errors in Table 3.2 illustrates the superb performance of the MIMO controller in tracking the desired trajectories. The MIMO adaptive SMC outperforms the SISO adaptive SMC in tracking desired AFR and engine speed under sampling and quantization imprecisions; however, the exhaust temperature tracking error increases using the MIMO adaptive controller compared to SISO adaptive SMC.

The reason of weaker performance of the MIMO SMC in tracking the desired exhaust temperature is that the magnitude of the $H S V$ for $T_{e x h}$ in $Z$ space is too small in comparison with those of AFR and engine speed. Thus, $T_{e x h}$ is considered as the less important state, compared to other states. The MIMO SMC puts more efforts on tracking AFR and engine speed, which are recognized as more important states to track [2]. Overall, the adaptive MIMO SMC has better performance in controlling the engine than those of the SISO adaptive and baseline SMCs. 
Table 3.2

Comparison between the baseline and MIMO adaptive SMCs in terms of mean error $\left(\frac{\int|e| d t}{\Delta t}\right)$ in tracking the desired engine trajectories.

\begin{tabular}{c|c} 
Controller & $\begin{array}{c}\text { Adaptive SMC } \\
\text { MIMO }\end{array}$ \\
\hline \hline AFR $[-]$ & $\begin{array}{c}0.113 \\
(-22.60 \% \downarrow)^{\dagger}\end{array}$ \\
\hline$T_{\text {exh }}\left[^{o} C\right]$ & $\begin{array}{c}6.9 \\
(-31.0 \% \downarrow)\end{array}$ \\
\hline Engine Speed, $\omega_{e}[R P M]$ & $\begin{array}{c}5 \\
(-41.2 \% \downarrow)\end{array}$ \\
\hline \hline Fuel & $\begin{array}{c}27.964 \\
(-0.16 \% \downarrow)\end{array}$ \\
\hline Consumption $[g]$ &
\end{tabular}

$\dagger$ The values inside the parentheses show the comparison between the MIMO adaptive SMC and the baseline SISO SMC tabulated in Table 3.1

Figure 3.7 shows the MIMO SMC performance in tracking desired AFR, exhaust temperature, and engine speed trajectories. When it turns to the AFR tracking, the MIMO adaptive controller illustrates a significantly better tracking behavior than the SISO adaptive SMC. This shows that the MIMO adaptive controller is able to track both desired air mass and fuel flow rates trajectories effectively and simultaneously with minimum errors (Figure 3.7 a). AFR and $\omega_{e}$ have less effects on $T_{\text {exh }}$ tracking when the MIMO adaptive controller is used compared to the SISO controller. Thus, the spikes in $T_{e x h}$ profile from the SISO SMC are removed in the MIMO controller (Figure 3.7-b). Unlike SISO SMC for engine speed regulation, the tracking performance is improved significantly using the MIMO SMC, whereas no overshoot is observed in the results from the MIMO controller (41.2\% improvement in tracking as shown in Figure $3.7 \mathrm{r}$ ). Moreover, Table 3.2 shows that the better performance 
of the MIMO adaptive SMC is achieved at no extra fuel cost compared to the SISO baseline and adaptive SMCs.
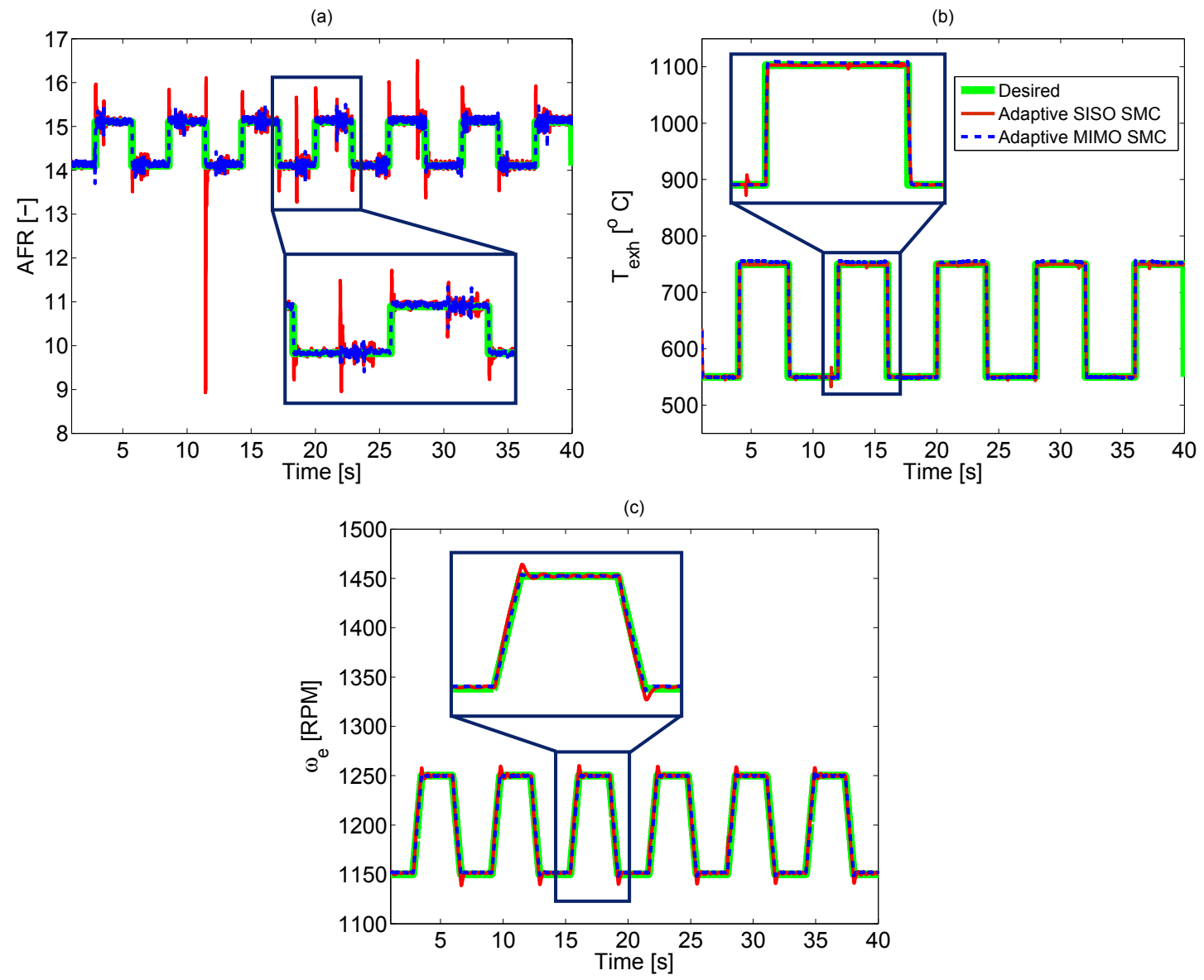

Figure 3.7: Real-time verification of the designed adaptive MIMO controller for tracking (a) AFR, (b) exhaust temperature, and (c) engine speed under ADC imprecisions (sampling time $=10 \mathrm{~ms}$, ECU update period $=$ $10 \mathrm{~ms}$, and quantization level $=10$ bit $)$.

The MIMO SMC is also verified for a set of desired trajectories that aim to reduce HC tailpipe emission during the cold start phase of the SI engine. Desired $A F R$ and engine speed trajectories are taken from the test results using the ECU of the Toyota engine. The exhaust temperature of $650{ }^{\circ} \mathrm{C}$ is chosen for the desired $T_{\text {exh }}$ using available experimental data for the engine [12]. Figure 3.8 shows the results 
(a)
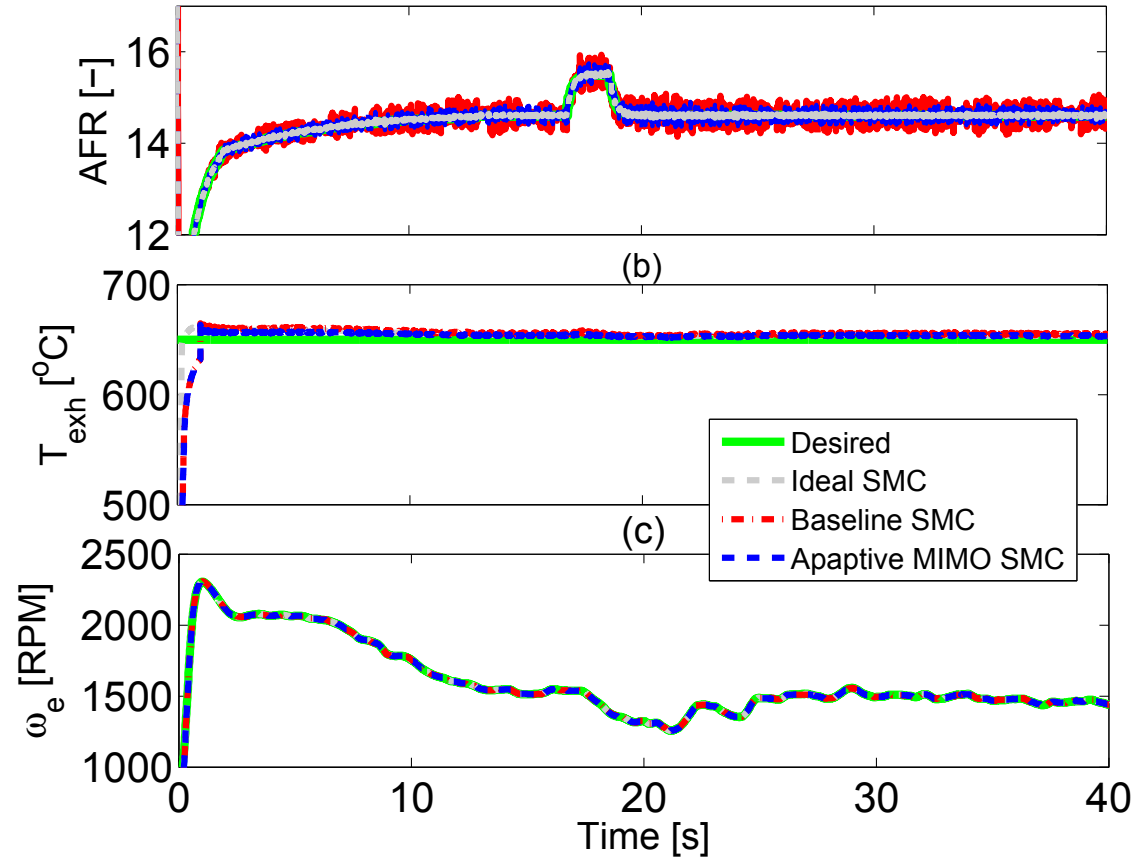

Figure 3.8: Real-time results of tracking the desired trajectories from ideal, baseline, and adaptive MIMO SMCs to control HC emission. (a) AFR, (b) exhaust gas temperature, and (c) engine speed. $10 \mathrm{~ms}$ sampling time and 10 bit quantization level. The data from the ideal SMC shows the baseline SMC performance with no ADC imprecisions.

of real-time testing the SMCs under ADC implementation imprecisions. Since the desired trajectories are smooth, the effects of ADC imprecisions on the controllers performance is mostly evident from AFR tracking results, while these imprecisions have less effects on the engine speed and $T_{e x h}$ tracking results. The baseline SMC will degrade from the ideal SMC results once sampling time increases and quantization level drops.

Figure 3.8 -a shows that using the proposed adaptation approach, the MIMO SMC is able to reduce AFR tracking error under ADC imprecisions. Given the large effect of AFR tracking on the engine-out $\mathrm{HC}$ emission, it can be observed from Figure 3.9 that 
the total amount of tailpipe $\mathrm{HC}$ produced during the cold start phase is significantlly reduced by using the adaptive MIMO SMC, compared to the baseline SMC.

As can be seen from Figure 3.9, the cumulative HC emission from the ideal SMC (i.e., the baseline SMC under no ADC imprecisions) is $2.43 \mathrm{~g}$, which can meet the minimum required $\mathrm{HC}$ emission level. However, once the same ideal controller is implemented on the real ECU under ADC imprecisions, the controller can no longer meet the target emission limit, as seen in the baseline SMC results in Figure 3.9 $\left(H C_{\text {cum }}=8.24 \mathrm{~g}\right)$. By incorporating the adaptation mechanism into the controller design and also converting the SISO structure to a MIMO structure based on nonlinear balanced realization, the new adaptive MIMO SMC is able to meet the target limit for $\mathrm{HC}$ emission by compensating the introduced ADC imprecisions and removing their adverse effects on the controller overall performance.

Overall, by utilizing the early model-based controller software verification and validation (Chapter 2), and easily verifiable controller design (this chapter) techniques, the actual iterations number in a traditional $\mathrm{V} \& \mathrm{~V}$ cycle can be decreased, which consequently helps to reduce the time and cost of the V\&V process. The proposed uncertainty-adaptive model-based controller design in this chapter aims to make completion of the $\mathrm{V} \& \mathrm{~V}$ process easier with less iterations. This is done through a robust controller design framework, which guarantees the satisfactory controller performance during the worst case scenarios, under different sampling and quantization levels. In 


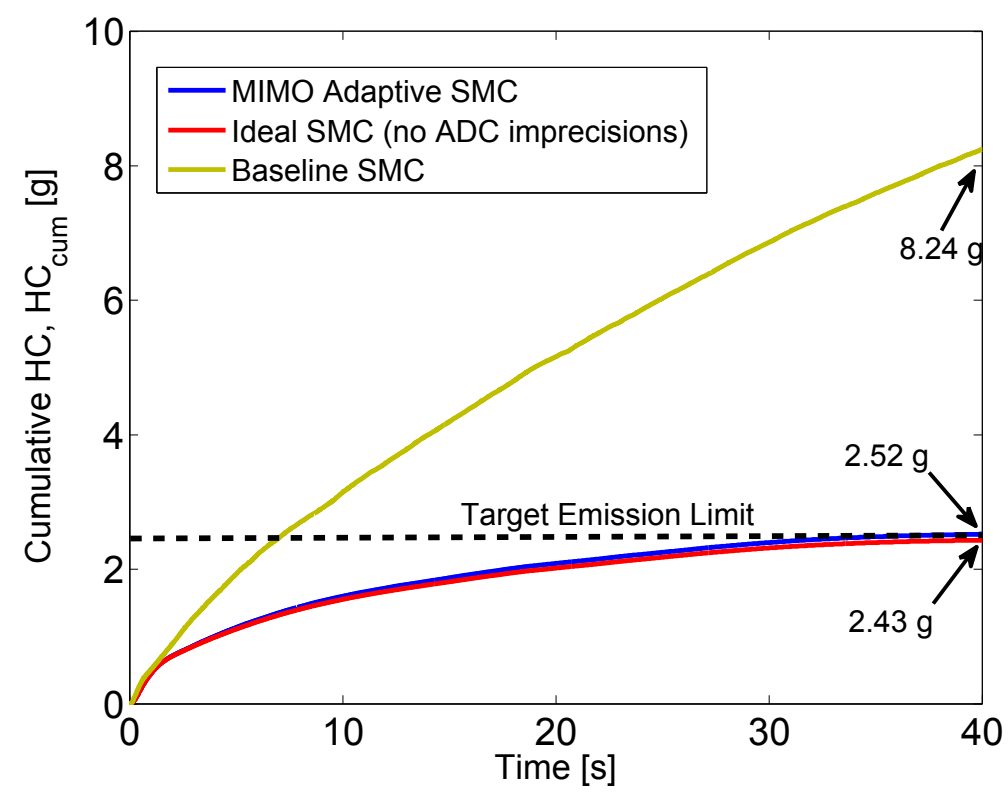

Figure 3.9: Cumulative tailpipe $\mathrm{HC}$ emission from ideal, baseline, and proposed adaptive MIMO SMCs. A cumulative $\mathrm{HC}$ emission limit of $2.5 \mathrm{~g}$ is selected for the first $40 \mathrm{sec}$ of the cold start phase in this study assuming that over $80 \%$ of the total HC emissions occur in the cold start phase of standard driving cycles $[82,83$.

the future chapters (4, 5, and 6), the uncertainty-adaptive easily verifiable controller design will be developed and investigated in discrete time, under ADC and modeling uncertainties.

\subsection{Summary and Conclusion}

An uncertainty-adaptive SMC design methodology was developed to improve the conventional SMC performance under two major implementation imprecisions including sampling and quantization. The Lyapunov's direct method was employed to prove the asymptotic stability of the proposed SMC which is updated at each time step by solving an adaptation law online. The uncertainty-adaptive SMC tries to adapt 
the controller by minimizing the tracking errors and overcome the ADC imprecisions. The proposed SISO uncertainty-adaptive SMC framework was examined on the engine control problem in SISO and MIMO structures. The goal was to track desired trajectories under implementation imprecisions. The uncertainty-adaptive SISO SMC showed better performance in exhaust temperature control by up to $50 \%$ reduction in the tracking error. However, due to the complexity of the engine plant, the developed adaptive SISO SMC could only provide small improvement (up to $16 \%$ and $2 \%$ ) in tracking the engine speed and AFR. On the other side, the MIMO SMC, which is designed based on a nonlinear balanced realization technique, is able to improve the tracking errors for engine speed and AFR up to $41 \%$ and $22 \%$, respectively. The realtime testing of the MIMO SMC on the PIL platform showed that the new adaptive controller is able to meet the control targets under ADC imprecisions. 


\section{Chapter 4}

\section{Discrete SMC with Robustness to Implementation Imprecisions ${ }^{1}$}

\subsection{Introduction}

It was shown in the literature [24, 88, 89] that a discrete sliding mode controller (DSMC), which explicitly includes the sampling time, shows better performance under sampling imprecisions compared to a continuous-time SMC that is digitally implemented. Quasi and baseline discrete sliding mode controllers were introduced and studied under implementation imprecisions in [88] and [89], respectively. The robustness of the DSMC design was enhanced by incorporating the maximum ADC uncertainty bounds on control inputs in [24]. An offline methodology for modeling and propagating the sampling and quantization uncertainties is proposed in [24]. In

\footnotetext{
${ }^{1} \mathrm{~A}$ major portion of the materials in this chapter has been published in the proceeding of 2016 American Control Conference 4] (doi:10.1109/ACC.2016.7526699) with the permission from IEEE as shown in Appendix D.
} 
this work, the overall uncertainty bounds on control signals are calculated using a linear noise propagation method. The predicted maximum uncertainty bounds are then incorporated into the formulation of the DSMC. The results showed that the proposed DSMC with maximum ADC uncertainty bounds can lead to better tracking performance in comparison with the baseline SMC/DSMC. As it was explained in Chapter 3, the incorporation of maximum ADC uncertainty bounds requires an ideal symbolic model of the controller which prevents the online uncertainty bounds calculation. Moreover, the results in [23] and [24] showed that the calculated maximum ADC uncertainty bounds, which are based on a worst case scenario, lead to conservative controller design and large control actions, which are not desired. To this end, in this chapter, an online technique is presented to predict and propagate sampling and quantization imprecisions on control signals. The new DSMC with predicted implementation imprecisions not only avoids the conservative controller design by using the new $\mathrm{ADC}$ uncertainty prediction technique, but also improves the robustness characteristics significantly compared to a baseline DSMC with no knowledge about the implementation uncertainties.

The contribution of this chapter is twofold. First, an online technique is developed and verified to predict and propagate uncertainties on measured signals by ADC in real-time. Second, a new discrete sliding mode controller (DSMC) design is constructed that incorporates the predicted uncertainties on measured signals. A generic 
algorithm is developed for both linear and nonlinear discrete systems for online estimation of the propagated uncertainties on control signals. The designed DSMCs are verified on a real ECU.

\subsection{DSMC with Predicted Uncertainties}

\subsubsection{Uncertainty Prediction on Measured Signal Due to $\mathrm{ADC}$}

Figure 4.1 illustrates the imprecisions caused by sampling on a measured analog signal. As shown in Figure 4.1, actual uncertainty $\left(\mu_{x_{s}}(i)\right)$ on a measured signal at each time step $(i)$ can be calculated with respect to the signal slope $(\Theta)$ as follows:

$$
\mu_{x_{s}}(i)=\Theta(i) \times T
$$

where $T$ is sampling time and $\Theta(i)$ is calculated with respect to the value of the sampled signal at the current and next time steps:

$$
\Theta(i)=\frac{x_{s}(i+1)-x_{s}(i)}{T}
$$

In Eq. 4.2, the value of $x_{s}(i+1)$ is unknown at $i^{\text {th }}$ time step, thus its value is predicted $\left(\hat{x}_{s}(i+1)\right)$ to obtain the uncertainty due to sampling. Here, the slope from the previous time instant $(i-1)$ is calculated and it is assumed that the slope at $i^{\text {th }}$ time step $(\Theta(i))$ is equal to the previous time instant $(\Theta(i-1))$ :

$$
\Theta(i-1)=\frac{x_{s}(i)-x_{s}(i-1)}{T} \stackrel{\Theta(i)=\Theta(i-1)}{\longrightarrow} \hat{x}_{s}(i+1)=T \times \Theta(i)+x_{s}(i)
$$




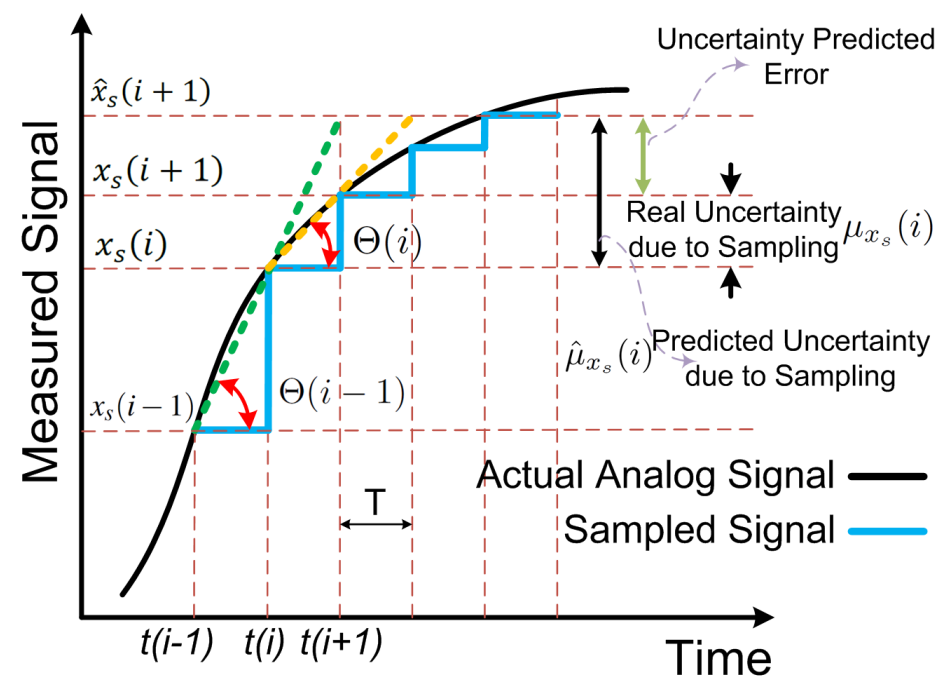

Figure 4.1: Uncertainty on a measured signal due to sampling.

which leads to:

$$
\hat{x}_{s}(i+1)=2 x_{s}(i)-x_{s}(i-1)
$$

Moreover, the uncertainty introduced by quantization $\left(\mu_{x_{q}}(i)\right)$ can be calculated at each time step by considering round-off error [1]:

$$
\mu_{x_{q}}(i)=\frac{1}{2} \frac{F S R}{2^{n}}
$$

Where $F S R$ is the full scale range of the measured signal and $n$ is the ADC's number of bits and represents the ADC resolution. Overall, the uncertainty on measured signals because of sampling and quantization at each time step can be expressed as follows using Eq. 4.1, 4.4, and 4.5):

$$
\mu_{x}(i)=\hat{\mu}_{x_{s}}(i)+\mu_{x_{q}}(i)=x_{s}(i)-x_{s}(i-1)+\frac{1}{2} \frac{F S R}{2^{n}}
$$

where $\hat{\mu}_{x_{s}}$ is the predicted uncertainty due to sampling with respect to the value of the predicted sampled signal at $i+1^{\text {th }}$ time step $\left(\hat{x}_{i+1}\right)$. The accuracy of Eq. (4.6) is 
sensitive to the magnitude of the changes in the signal's slope. Moreover, the accuracy of the uncertainty estimation depends on the ADC quantization level. Lower ADC resolution leads to poor uncertainty estimation. During the time interval between two time steps, output of a controller is constant, therefore the predicted value for ADC imprecisions at each time step can be assumed to be constant over the next time interval. In other words, Eq. 4.6 determines ADC uncertainty prediction at each time step till the next time step.

\subsubsection{DSMC Design and Uncertainty Propagation}

Sliding mode controller design converts a complicated dynamical system into a firstorder system through a sliding surface transformation. A discrete sliding mode control design approach is adapted from [89] in this study. Sliding surface vector $(S)$ is defined as the difference between desired $\left(X_{d}\right)$ and measured signal $(X)$ vectors at each time step $(i)$ as follows:

$$
\left\{\begin{array}{l}
S(i)=X(i)-X_{d}(i) \\
S(i+1)=X(i+1)-X_{d}(i+1)
\end{array}\right.
$$

The control input $U(i)$ is obtained according to the following sliding surface reaching law:

$$
|S(i+1)| \leq \rho|S(i)|
$$


where $\rho$ is the diagonal matrix of tunable DSMC parameters. In the following, the DSMC is first formulated for linear systems to estimate the propagated uncertainties on control signals analytically. Next, the DSMC will be extended to a general class of affine nonlinear systems, and the uncertainty propagation for nonlinear systems will be discussed.

Linear Systems: Any linear system with the following state equations can be discretized using a first order Euler approach [89]:

$$
\dot{X}=A X(t)+B U(t) \stackrel{\dot{x}=\frac{x(i+1)-x(i)}{T}}{\longrightarrow} X(i+1)=(T A+I) X(i)+(T B) U(i)
$$

where $x \subset X \in \mathbb{R}^{r}, u \subset U \in \mathbb{R}^{h}$, and $I \in \mathbb{R}^{r \times r}$ are the state vector, the control input vector, and identity matrix, respectively. Predicted uncertainty on measured signals from Eq. (4.6) is assumed to be the difference between measured signals before and after $\operatorname{ADC}\left(\mu_{x}=\bar{x}-x\right)$, where $\bar{x}$ is the actual measured signal before ADC. The diagonal matrix of tunable DSMC parameters for the sliding surfaces' convergence is $\rho=\operatorname{diag}\left[\rho_{1}, \ldots, \rho_{r}\right]$, where $0<\rho_{1, \ldots, r}<1$ constraint should be met to satisfy stability conditions of the controller [89]. In the worst case, where $|S(i+1)|=\rho|S(i)|$, Eq. (4.8) can be expanded with respect to Eq. (4.9):

$$
(T A+I) X(i)+(T B) U(i)-X_{d}(i+1)=\rho\left(X(i)-X_{d}(i)\right)
$$

Assuming a common class of single-input single-output (SISO) linear systems in which $r=h$, and $B$ is diagonal, Eq. 4.10 can be solved for extracting control input vector $U$ as follows: 


$$
U(i)=B^{-1}\left(\frac{1}{T}\left[(\rho-I) X(i)-\rho X_{d}(i)+X_{d}(i+1)\right]-A X(i)\right)
$$

Eq. (4.11) calculates the control input vector at each time step for a set of SISO DSMCs, in which for each state variable, a sliding surface is defined and it is assumed that a unique control input, either physical or synthetic, exists for every single sliding surface that drives the corresponding state to the desired value in a finite time. For the ideal controller where there is no ADC imprecisions on the measured signals $(X)$, the baseline DSMC can be established as follows:

$$
\bar{U}(i)=B^{-1}\left(\frac{1}{T}\left[(\rho-I) \bar{X}(i)-\rho X_{d}(i)+X_{d}(i+1)\right]-A \bar{X}(i)\right)
$$

where $\bar{X}$ and $\bar{U}$ are the state and control input vectors of an ideal DSMC which aims to control the same linear system in Eq. (4.9) with respect to the same desired trajectories $\left(X_{d}\right)$ in Eq. 4.7). Since the only discrepancy between ideal DSMC (Eq. 4.12) and DSMC under test (Eq. (4.11) is the introduced imprecisions from ADC, the difference in the control input vectors from Eq. (4.11) and (4.12) is assumed to be the propagated uncertainty vector on control input $\left(\mu_{U}\right)$ due to ADC imprecisions:

$$
\mu_{U}=\operatorname{diag}\left[\bar{u}_{1}-u_{1}, \ldots, \bar{u}_{r}-u_{r}\right]
$$

Since it was defined that $\bar{X}=\mu_{X}+X$, and $\mu_{X}=\left\{\mu_{x_{1}}, \mu_{x_{2}}, \ldots, \mu_{x_{r}}\right\}$ could be predicted at each time step according to Eq. 4.6], the following relationship can be utilized to predict propagated ADC uncertainties on control input of the DSMC under test by expanding Eq. (4.13): 


$$
\mu_{U}(i)=B^{-1}\left(\frac{1}{T}\left[(\rho-I) \mu_{X}(i)\right]-A \mu_{X}(i)\right)
$$

Nonlinear Systems: Similar to linear systems, the first order Euler approximation is used to discretize the following general class of affine nonlinear systems:

$$
\dot{X}=f(X(t))+g U(t) \stackrel{\dot{x}=\frac{x(i+1)-x(i)}{T}}{\longrightarrow} X(i+1)=X(i)+f T+g U(i) T
$$

where $X, f, U \in \mathbb{R}^{r}$, and $I, g \in \mathbb{R}^{r \times r}$. The coefficient matrix $g$ is a non-singular square matrix and $f$ represents part of system dynamics which does not depend on the inputs. The baseline DSMC can be formulated for this general class of nonlinear systems with the sliding surface function and reaching law described in Eq. (4.7) and (4.8) as follows:

$$
U(i)=\frac{g^{-1}}{T}\left[(\rho-I) X(i)-\rho X_{d}(i)-f T+X_{d}(i+1)\right]
$$

where $\rho \in \mathbb{R}^{r \times r}$. Unlike linear systems in which the predicted uncertainties on measured signals can be propagated on control signals using Eq. 4.14, in nonlinear systems it is not possible to conduct the noise propagation analytically. Figure 4.2 shows our proposed approach to estimate propagated uncertainties on control signals for the nonlinear system described in Eq. 4.15). The uncertainty prediction mechanism contains two auxiliary baseline DSMCs, including a virtual ideal DSMC and the DSMC under test. Desired trajectories for both auxiliary DSMCs are the same. The feedback signals to the DSMC under test are measured signals after $\operatorname{ADC}(X)$, while the inputs to the virtual ideal DSMC are the estimated measured signals before ADC $(\hat{\bar{X}}) . \hat{\bar{X}}$ is estimated according to the real values of measured signals after ADC and 


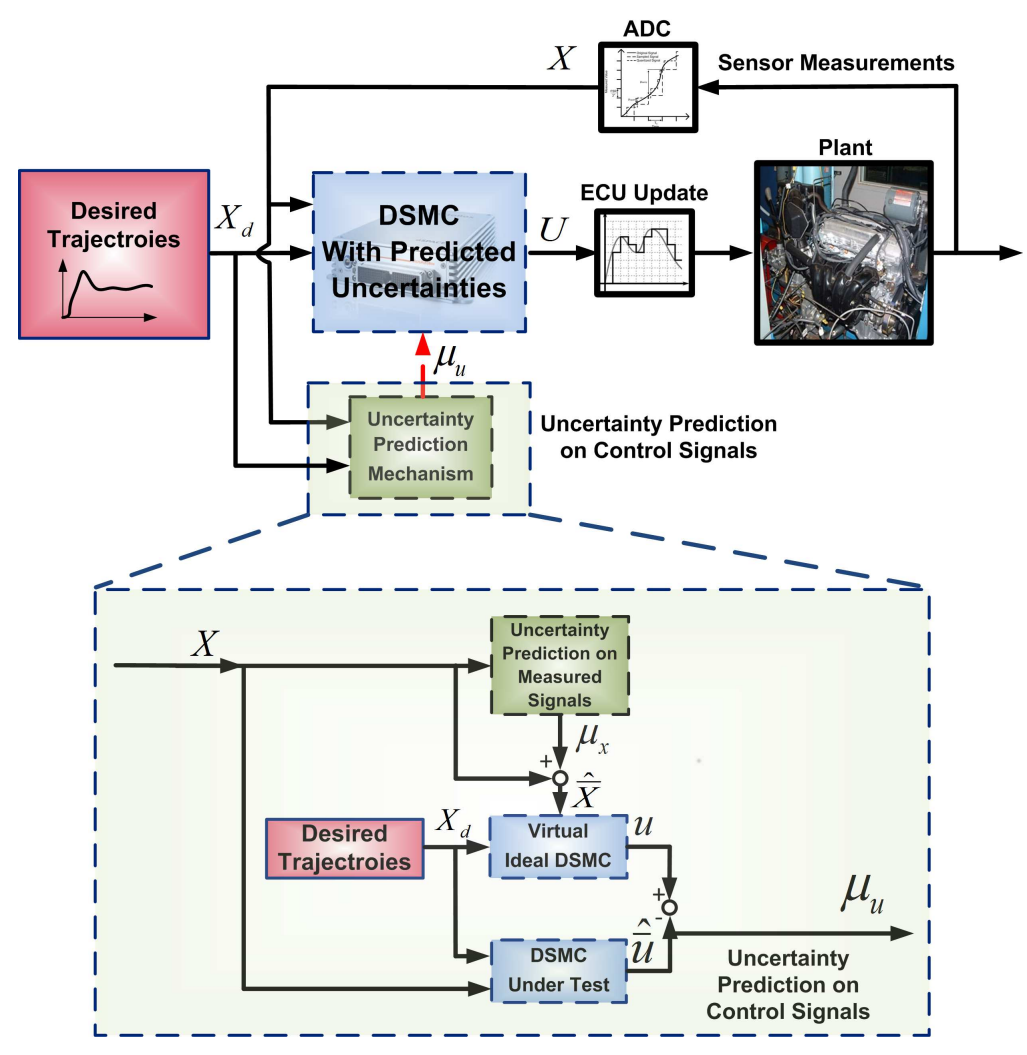

Figure 4.2: Schematic of the DSMC with online estimations of uncertainties on measured and control signals.

predicted ADC uncertainties on measured signals:

$$
\hat{\bar{X}}=\mu_{X}+X
$$

Since the only difference between the DSMC under test and the virtual ideal DSMC is the ADC uncertainties on measured signals, the propagated uncertainty vector on control signals is the difference between control signals of these two controllers as follows:

$$
\mu_{U}=\operatorname{diag}\left[\hat{\bar{u}}_{1}-u_{1}, \ldots, \hat{\bar{u}}_{1}-u_{r}\right]
$$

where $\hat{\bar{u}}_{1, \ldots, r}$ are the estimated control inputs from the ideal DSMC $(\bar{u})$ according to the predicted ADC uncertainties on measured signals. 


\subsubsection{Inclusion of Propagated Uncertainties into the DSMC}

Structure of the baseline DSMC (Eq. 4.11) and (4.16) is modified by inclusion of estimated uncertainties on control signals into the DSMC formulation:

$$
U^{\bmod }(i)=U(i)+\delta(i)
$$

where $U^{\text {mod }}$ is the modified controller input and $U$ is calculated with respect to Eq. (4.11) and (4.16) for linear and nonlinear systems, respectively. $\delta(i)$ is included to compensate for hardware imprecisions. $\delta(i)$ is obtained according to the predicted uncertainties on control signals as follows:

$$
\delta(i)=\mu_{U}(i) S(i)
$$

By inclusion of propagated ADC uncertainties on control signals $\left(\mu_{U}\right)$, the baseline DSMC for a linear $(l)$ system is modified as follows:

$$
U^{m o d, l}(i)=B^{-1}\left(\frac{1}{T}\left\{(\rho-I) X(i)-\rho X_{d}(i)+X_{d}(i+1)\right\}-A X(i)\right)-\mu_{U}(i) S(i)
$$

In addition, the baseline DSMC for the affine nonlinear $(n l)$ system in Eq. (4.15) is modified to:

$$
U^{m o d, n l}(i)=\frac{g^{-1}}{T}\left[(\rho-I) X(i)-\rho X_{d}(i)-f T+X_{d}(i+1)\right]-\mu_{U}(i) S(i)
$$




\subsection{Case Studies}

Here, application of the proposed method in Section 4.2 .2 is demonstrated for one linear and one nonlinear case studies.

\subsubsection{Linear Case Study: DC Motor Speed Control}

DC motor is a common actuator for control applications that require rotary and transitional motions. The electric equivalent circuit of the armature and the free-body diagram of the rotor are shown in Figure 4.3. For speed regulation of a DC motor, the control input is voltage $(V)$ to the motor's armature and the output is rotation speed $(\theta)$ of the shaft. Assuming a constant magnetic field and linear relationship between motor torque and armature current $(\mathcal{I})$, by choosing the rotor speed and current as the state variables, the following linear time-invariant state-space representation can be used to describe the dynamics of the DC motor [90]:

$$
\begin{array}{r}
\theta(i+1)=T\left(\frac{k_{m}}{J} \mathcal{I}(i)-\frac{k_{f}}{J} \theta(i)+\frac{1}{J} \Gamma\right)+\theta(i) \\
\mathcal{I}(i+1)=T\left(-\frac{k_{b}}{L} \theta(i)-\frac{R}{L} \mathcal{I}(i)+\frac{1}{L} V(i)\right)+\mathcal{I}(i)
\end{array}
$$

where $J$ is the rotor's moment of inertia, $R$ is the electrical resistance, $L$ is the electric inductance, $\Gamma$ is the torque on the rotor, $k_{f}$ is the mechanical damping, $k_{m}$ is the motor torque constant, and $k_{b}$ is the electromotive force constant. The DC motor model constants are listed in the Appendix A. 


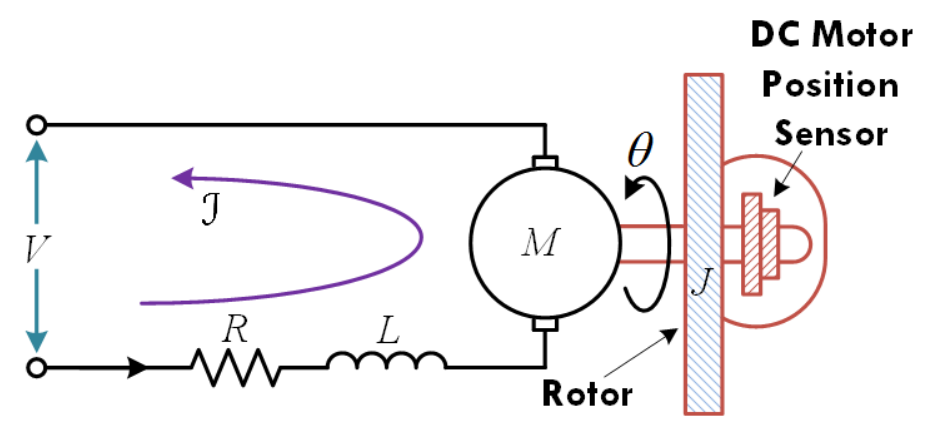

Figure 4.3: Schematic of the modeled DC motor.

Performance of the proposed uncertainty prediction technique is investigated for the DC motor, under $50 \mathrm{~ms}$ of sampling time and 10 bit of quantization level. $\theta$ and $i$ are feedback signals that go through ADC before going to the DC motor controller. Simulations are done in MATLAB/Simulink® that allows to test the controller in an MIL platform against sampling and quantization imprecisions. Figure 4.4 shows the ADC uncertainty prediction results on the shaft speed and the current of the armature circuit. In this figure, the term "measured" denotes the signal after ADC in the MIL setup in MATLAB. The accuracy of the uncertainty prediction technique is shown in Table 4.1 in terms of mean error and standard deviation. The small error values in Table 4.1 confirm the capability of the proposed method to estimate ADC uncertainty on measured signals for the DC motor case study.

Table 4.1

Mean $(\bar{e})$ and Standard Deviation $\left(\sigma_{e}\right)$ of ADC Uncertainty Prediction Errors for the DC Motor Model.

\begin{tabular}{lcc}
\hline \hline & $\bar{e}$ & $\sigma_{e}$ \\
\hline Motor Speed, $\theta[\mathrm{rad} / \mathrm{sec}]$ & 0.002 & 0.003 \\
Current, $i[A]$ & 0.045 & 0.053 \\
\hline \hline
\end{tabular}


(a)

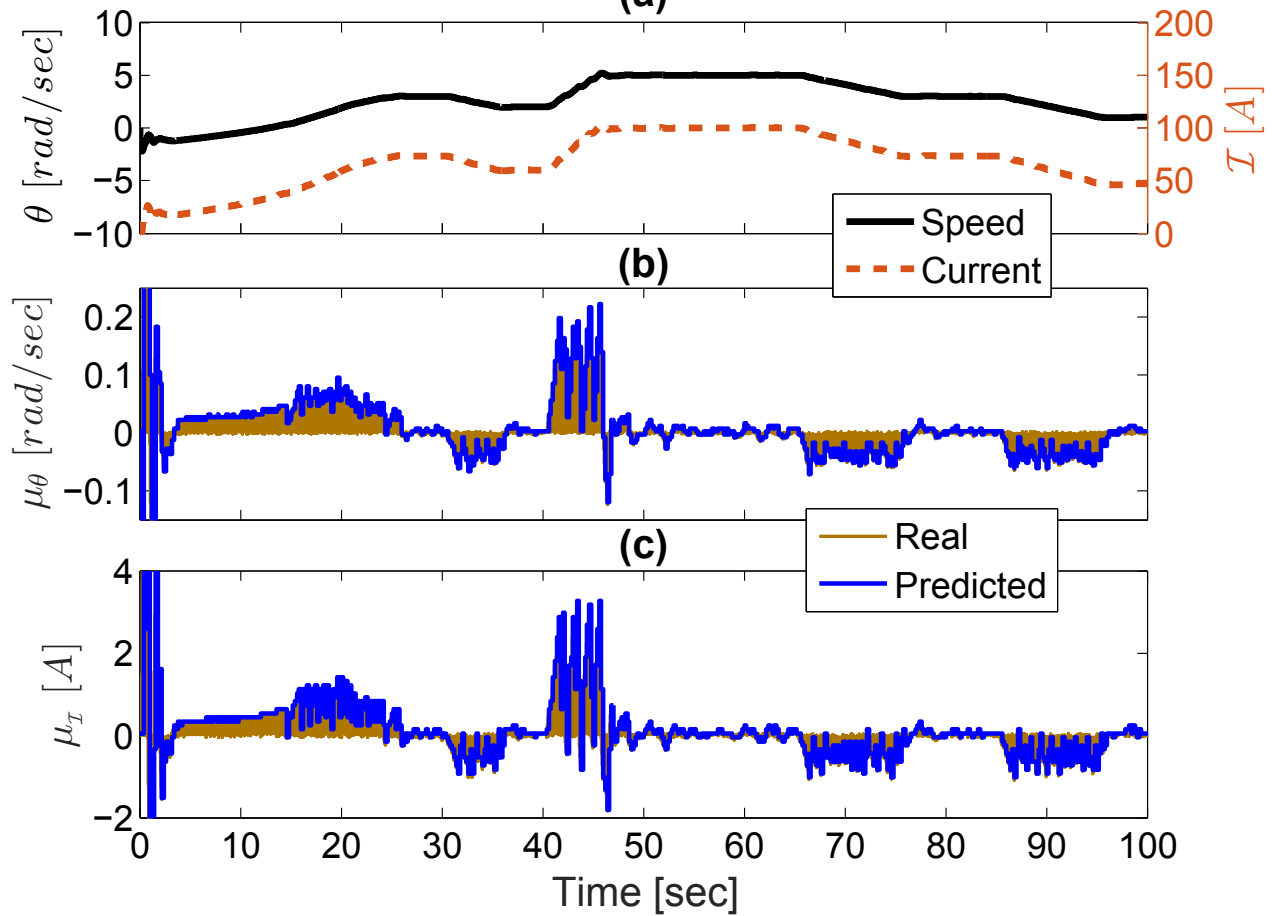

Figure 4.4: Comparison of actual and predicted uncertainties due to sampling and quantization. (a) measured signals (i.e., signal after ADC), (b) actual and predicted uncertainties on measured speed signal, and (c) actual and predicted uncertainties on current signal. (200 ms sampling time and 10-bit quantization level).

A DSMC is designed to regulate the DC motor rotational speed with respect to desired speed profile $\left(\theta_{d}\right)$ under implementation imprecisions. The first sliding surface is defined as the error in tracking desired speed profile $\left(s_{1}=\theta-\theta_{d}\right)$. Since there is no direct control input on DC motor rotational speed, $\mathcal{I}_{d}$ is defined as the synthetic control input for controlling the shaft speed. $\mathcal{I}_{d}$ is used to define the second sliding surface $\left(s_{2}=\mathcal{I}-\mathcal{I}_{d}\right)$ in which the control input is voltage.

Eq. (4.21) is used to derive control input equations for the modified DSMC with incorporated predicted uncertainties. To this end, the DC motor model is first discretized 
according to Eq. (4.9). Next, the modified DSMC with predicted implementation uncertainties on control signals for the DC motor speed control problem is calculated as follows:

$$
\begin{array}{r}
\mathcal{I}_{d}(i)=\frac{J}{k_{m}}\left(\frac { 1 } { T } \left[\rho_{1}\left(\theta(i)-\theta_{d}(i)\right)+\theta_{d}(i+1)\right.\right. \\
\left.-\theta(i)]+\frac{k_{f}}{J} \theta(i)-\frac{1}{J} \Gamma\right)-\mu_{\mathcal{I}_{d}}(i)\left[\theta(i)-\theta_{d}(i)\right] \\
V(i)=L\left(\frac{1}{T_{s}}\left[\rho_{2}\left(\mathcal{I}(i)-\mathcal{I}_{d}(i)\right)+\mathcal{I}_{d}(i+1)-\mathcal{I}(i)\right]\right. \\
\left.+\frac{k_{b}}{L} \theta(i)+\frac{R}{L} i(i)\right)-\mu_{V}(i)\left[\mathcal{I}(i)-\mathcal{I}_{d}(i)\right]
\end{array}
$$

where $\mu_{\mathcal{I}_{d}}$ and $\mu_{V}$ denote the estimations of propagated ADC uncertainties on control signals and are calculated according to Eq. 4.14 as follows:

$$
\begin{gathered}
\mu_{\mathcal{I}_{d}}(i)=\frac{J}{T k_{m}}\left(\left(\rho_{1}-1\right) \mu_{\theta}(i)\right)+\frac{k_{f}}{k_{m}} \mu_{\theta}(i) \\
\mu_{V}(i)=\frac{L}{T}\left(\left(\rho_{2}-1\right) \mu_{\mathcal{I}}(i)\right)+k_{b} \mu_{\theta}(i)+R \mu_{\mathcal{I}}(i)
\end{gathered}
$$

$\mu_{\theta}$ and $\mu_{\mathcal{I}}$ are predicted uncertainties on measured signals that are calculated using Eq. (4.6) and were previously shown in Figure 4.4. Performance of the baseline continuous-time SMC and proposed DSMC with incorporated implementation uncertainties are shown in Figure 4.5 for tracking the desired speed profile. As it can be observed, the DSMC with predicted uncertainties is able to significantly improve the tracking performance compared to the baseline SMC (the tracking error is reduced by $71.2 \%)$. 


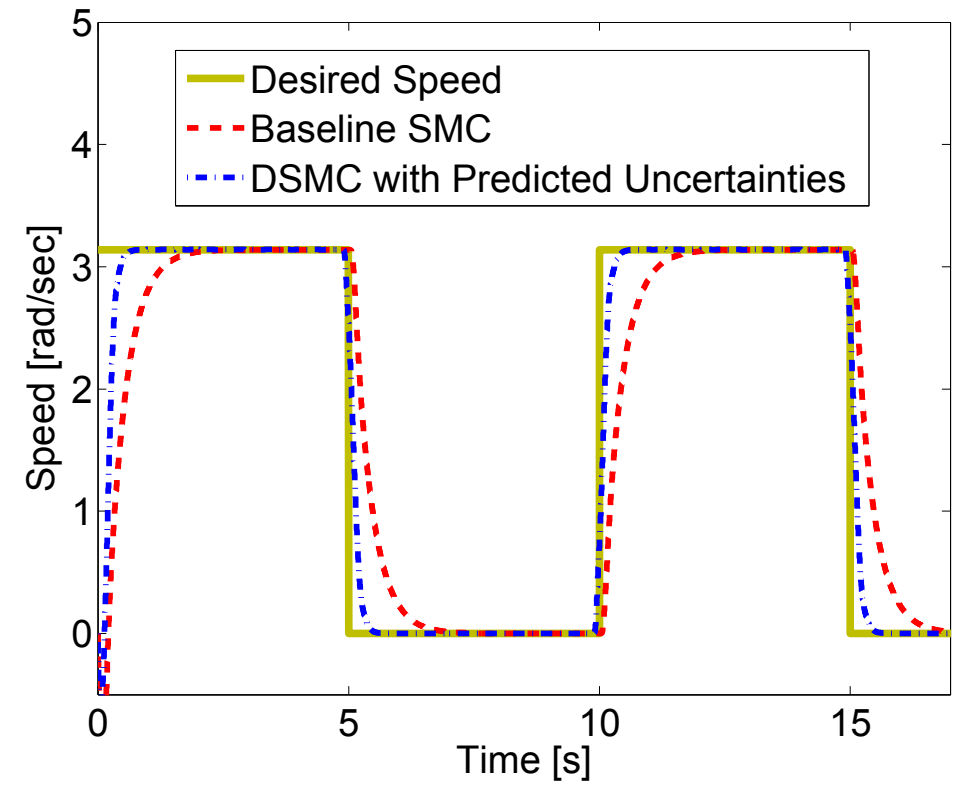

Figure 4.5: DC motor speed control using the baseline SMC and the modified DSMC (sampling time: $50 \mathrm{~ms}$, quantization level: $10 \mathrm{bit}$ ).

\subsubsection{Nonlinear Case Study: Automotive Engine Control}

In this section, the proposed DSMC with predicted ADC imprecisions is designed and evaluated for the highly nonlinear SI engine model during cold start from Section 2.3 in Chapter 2. Discrete state equations of the engine model are shown below:

$$
\begin{gathered}
T_{e x h}(i+1)=T_{e x h}(i)+\frac{T}{\tau_{e}}\left[(7.5 \Delta(i)+600) A F I(i)-T_{e x h}(i)\right] \\
\dot{m}_{f}(i+1)=\dot{m}_{f}(i)+\frac{T}{\tau_{f}}\left[\dot{m}_{f c}(i)-\dot{m}_{f}(i)\right] \\
\omega_{e}(i+1)=\omega_{e}(i)+\frac{T}{J} T_{E}(i) \\
m_{a}(i+1)=m_{a}(i)+\left[\dot{m}_{a} i(i)-\dot{m}_{a} o(i)\right] T
\end{gathered}
$$


Similar to continuous-time SMC from Chapter 3, four sliding surfaces for the engine controller are defined as follows:

$$
\begin{gathered}
s_{1}=T_{e x h}-T_{e x h, d}, \quad s_{2}=\dot{m}_{f}-\dot{m}_{f, d} \\
s_{3}=\omega_{e}-\omega_{e, d}, \quad s_{4}=m_{a}-m_{a, d}
\end{gathered}
$$

Eq. 4.22 is employed in the next step to derive control input equations for the DSMC with predicted implementation uncertainties. The resulting DSMC with incorporated uncertainties on control signals is expressed in the following equations:

$$
\begin{gathered}
\Delta(i)=\frac{\tau_{e}}{7.5 A F I . T}\left[\frac{T}{\tau_{e}}\left(-600 A F I+T_{e x h}(i)\right)\right. \\
\left.+\left(\rho_{1}-1\right) s_{1}(i)+T_{e x h, d}(i+1)-T_{e x h, d}(i)\right]-\mu_{\Delta}(i) s_{1}(i) \\
\dot{m}_{f c}(i)=\frac{\tau_{f}}{T}\left[\frac{T}{\tau_{f}} \dot{m}_{f}(i)+\left(\rho_{2}-1\right) s_{2}(i)\right. \\
\left.+\dot{m}_{f, d}(i+1)-\dot{m}_{f, d}(i)\right]-\mu_{\dot{m}_{f c}}(i) s_{2}(i) \\
m_{a, d}(i)=\frac{J}{30,000 T}\left[\frac{T}{J}\left(100+0.4 \omega_{e}(i)\right)+\left(\rho_{3}-1\right) s_{3}(i)\right. \\
\left.+\omega_{e, d}(i+1)-\omega_{e, d}(i)\right]-\mu_{m_{a, d}}(i) s_{3}(i) \\
\dot{m}_{a i}(i)=\frac{1}{T}\left[\dot{m}_{a o}(i) T+\left(\rho_{4}-1\right) s_{4}(i)+m_{a, d}(i+1)\right. \\
\left.-m_{a, d}(i)\right]-\mu_{\dot{m}_{a i}}(i) s_{4}(i)
\end{gathered}
$$


where $\mu_{\Delta}, \mu_{\dot{m}_{f c}}, \mu_{m_{a, d}}$, and $\mu_{\dot{m}_{a i}}$ are the estimations of propagated ADC uncertainties on control signals which are computed according to the approach explained in Figure 4.2. Estimation of propagated ADC uncertainties on control signals requires the knowledge of ADC uncertainty on measured signals $\left(\mu_{T_{e x h}}, \mu_{\dot{m}_{f}}, \mu_{\omega_{e}}\right.$, and $\left.\mu_{m_{a}}\right)$ which are predicted using Eq. (4.6). Table 4.2 summarizes the results of online ADC uncertainty prediction on the states of the engine model in terms of mean and standard deviation errors. It can be observed that the proposed uncertainty prediction approach, described in Eq. 4.6, is able to estimate the introduced uncertainties accurately.

Figure 4.6 shows the performance of the designed controllers in tracking desired trajectories for AFR, exhaust gas temperature, and engine speed ( $N[R P M])$ under ADC imprecisions (10 ms sampling time and 10 bit of quantization), respectively. Baseline DSMC has the same equations as Eq. 4.334.36) excluding the propagated implementation uncertainties $\left(\mu_{\Delta}=\mu_{\dot{m}_{f c}}=\mu_{m_{a, d}}=\mu_{\dot{m}_{a i}}=0\right)$.

Table 4.2

Mean $(\bar{e})$ and Standard Deviation $\left(\sigma_{e}\right)$ errors for Predicting the ADC Uncertainty for the Engine Model's Measured Signals.

\begin{tabular}{lcc}
\hline \hline & $\bar{e}$ & $\sigma_{e}$ \\
\hline$m_{a}[\mathrm{~kg}]$ & $2.060 \times 10^{-5}$ & $2.321 \times 10^{-5}$ \\
$\dot{m}_{f}[\mathrm{~kg} / \mathrm{sec}]$ & $2.337 \times 10^{-6}$ & $2.732 \times 10^{-6}$ \\
$\omega_{e}[\mathrm{rad} / \mathrm{sec}]$ & 0.124 & 0.145 \\
$T_{\text {exh }}\left[{ }^{\circ} \mathrm{C}\right]$ & 0.5 & 0.5 \\
\hline \hline
\end{tabular}

The mean and standard deviations of the tracking errors are given in Table 4.3. The 

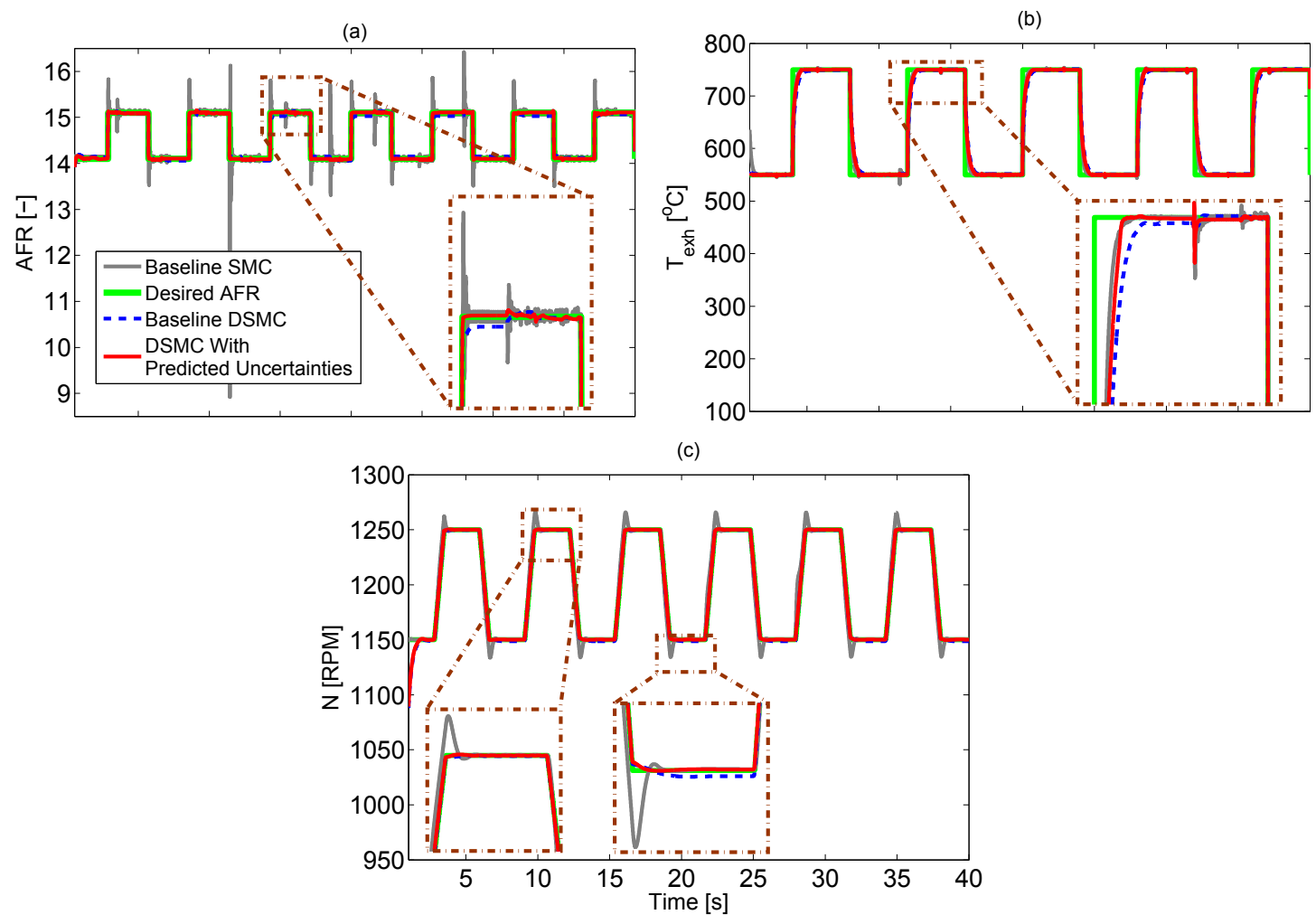

Figure 4.6: Results of engine control under ADC imprecisions: (a) AFR, (b) exhaust gas temperature, and (c) engine speed. (10 ms sampling time and 10 bit quantization level)

mean tracking errors were reduced compared to the baseline SMC by $42 \%$ to $93 \%$ using DSMC with predicted uncertainties, while these improvements are $18 \%$ to $88 \%$ by using the baseline DSMC. The standard deviations of the tracking errors from the baseline and modified DSMCs for both AFR and engine speed were also reduced by more than $90 \% . \sigma_{e}$ for $T_{e x h}$ tracking is reduced by $50 \%$ by using the proposed DSMC, compared to the baseline SMC. 


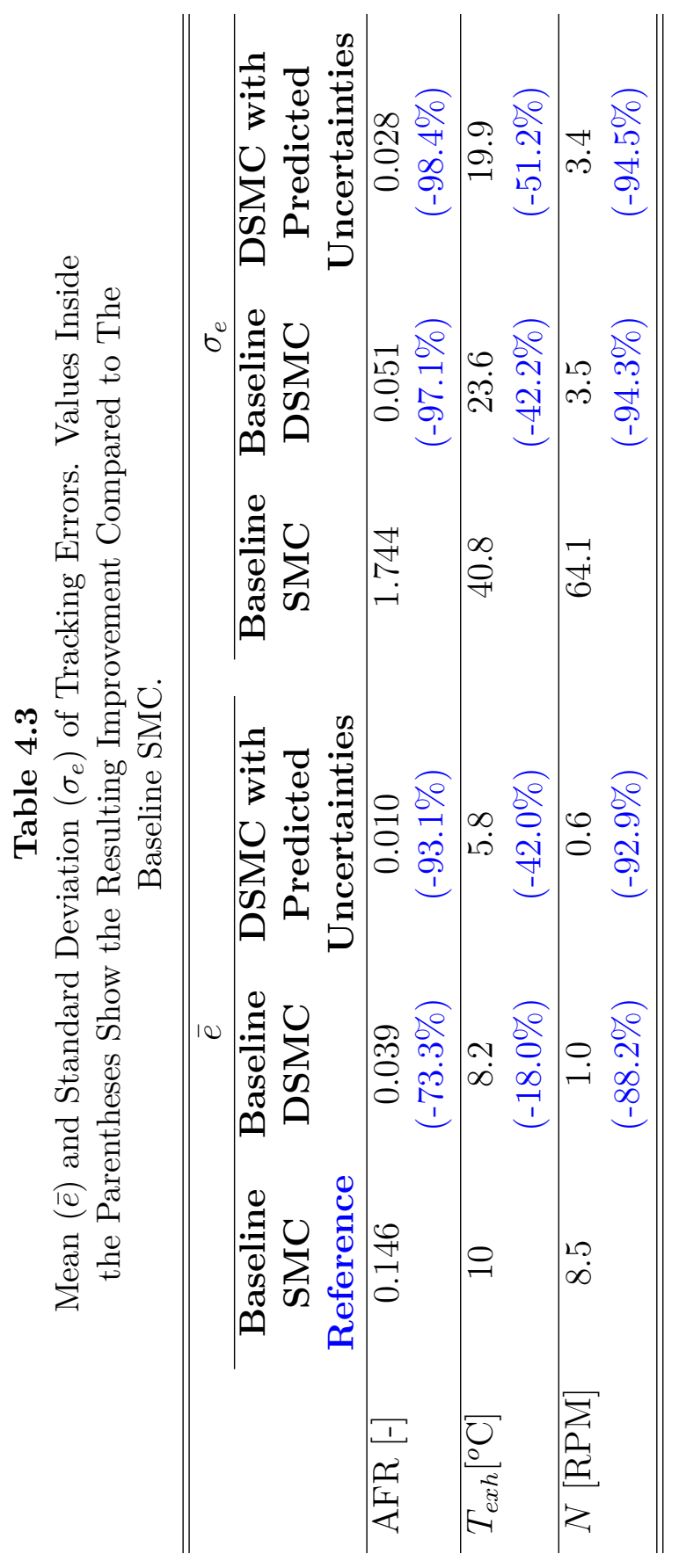


Figure 4.6 a shows that the baseline SMC cannot track the desired AFR properly. The baseline DSMC improves the AFR tracking by $73 \%$ compared to the baseline SMC. Although the baseline DSMC shows significant improvement compared to the baseline SMC, it still cannot track the desired AFR profile accurately. Once the predicted uncertainty is incorporated into the DSMC, the AFR tracking error is reduced by $20 \%$ compared to the baseline DSMC. This results in accurate tracking of desired AFR trajectory against ADC imprecisions.

Figure 4.6 b and Table 4.3 show that by incorporating the predicted implementation uncertainties, the $T_{\text {exh }}$ tracking error can be reduced by $42 \%$ compared to the baseline SMC. Controlling the engine speed using the baseline SMC causes overshoots when the desired speed profile drops or rises sharply. It can be observed from Figure 4.6 c that the baseline DSMC is able to remove the overshoots and reduces the mean tracking error by $88 \%$; however, steady state error still exists. The steady state tracking error of the engine speed is overcome by incorporating the predicted uncertainties on $\dot{m}_{a i}$ and $m_{a, d}$ into the DSMC. As can be seen from Figure 4.6-c and Table 4.3 , the modified DSMC is able to remove the steady state error in tracking the desired speed trajectory and reduces the mean tracking error by about $93 \%$, compared to the baseline SMC. 


\subsection{DSMC Real-Time Verification}

The designed DSMCs are tested in the processor-in-the-loop (PIL) setup previously shown in Figure 3.6 in Chapter 3 . Figure 4.7 and 4.8 show the results of real-time PIL testing of the proposed DSMCs with incorporated uncertainties on control signals for the DC motor and the engine case studies, respectively. The results verify that (i) the designed DSMCs are able to track all the desired trajectories under implementation imprecisions, and (ii) the DSMCs are computationally efficient for real-time operation.

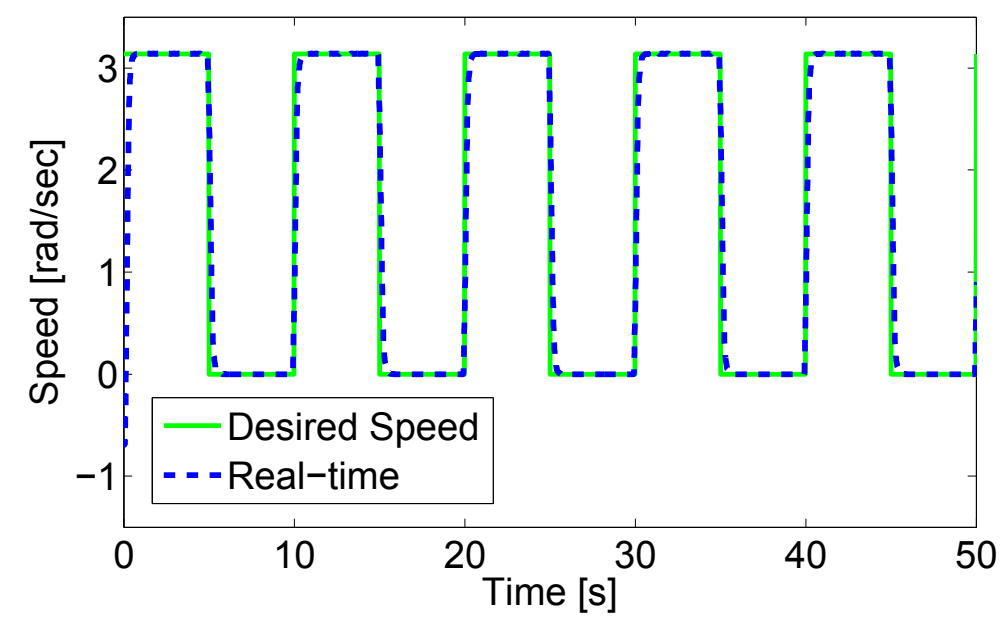

Figure 4.7: Results of DSMC real-time verification for the DC motor speed control (50 ms sampling time and 10 bit quantization level).

\subsection{Summary and Conclusion}

An online methodology was proposed and verified for predicting the introduced ADC implementation imprecisions on measured signals. A generic DSMC was formulated 
(a)

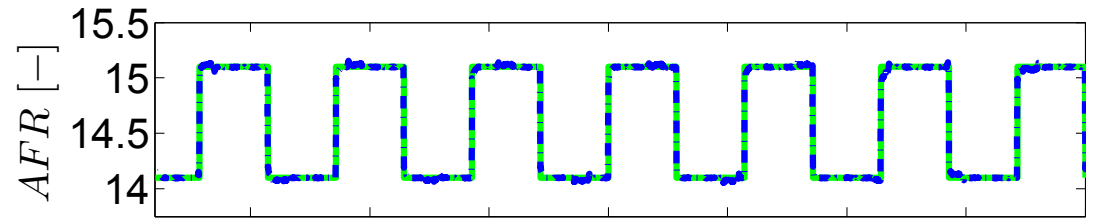

(b)

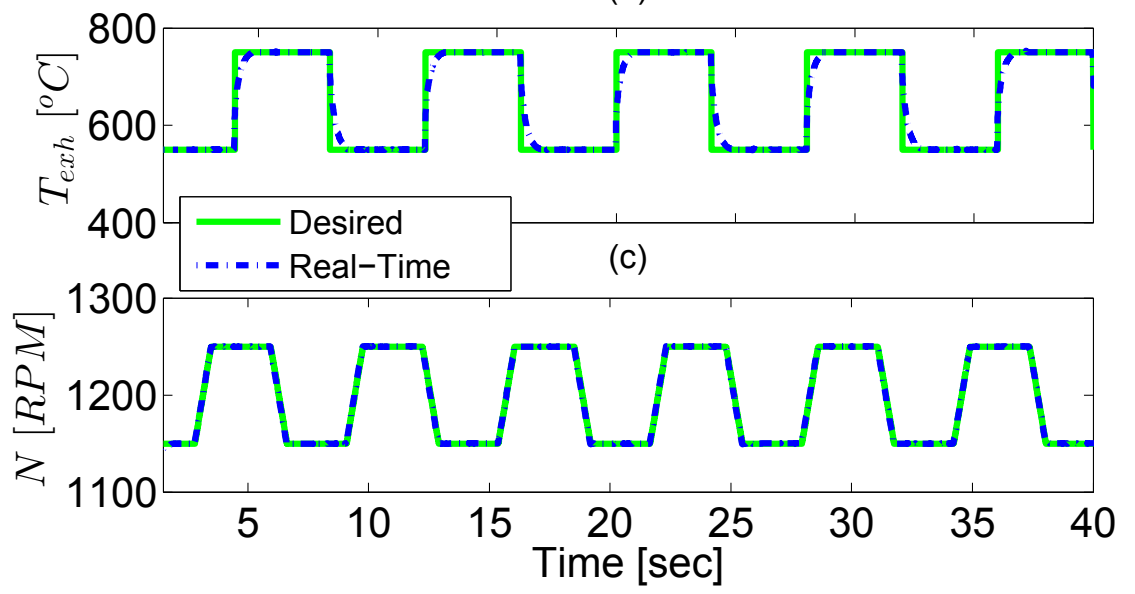

Figure 4.8: Real-time verification for the engine control using the proposed DMSC (10 ms sampling time and 10 bit quantization level).

for linear and nonlinear systems to track desired trajectories. The predicted imprecisions on measured signals were first propagated on control signals and they were then incorporated into the DSMC. A PIL testing was employed to verify the real-time performance of the proposed DSMC for two case studies (DC motor speed regulation and automotive engine control problem). Real-time testing results showed that the new DSMC design is able to improve the tracking performance under sampling and quantization imprecisions by $40-90 \%$, compared to a conventional SMC design. 


\section{Chapter 5}

\section{First Order SISO Adaptive Discrete Sliding Mode Control ${ }^{1}$}

\section{$5.1 \quad$ Introduction}

The capabilities of SMC and DSMC in handling implementation imprecisions were studied in Chapters 3 and 4 . In addition to implementation imprecisions, SMC allows for dealing with uncertainties, un-modeled dynamics, and disturbances. Handling the modeling uncertainty in SMC design has been the subject of previous works in the literature [56, 57, 58, 60, 61, 62]. The adaptive DSMC formulation from [62] presents a generic solution for removing a multiplicative type of model uncertainty for a general class of nonlinear systems. However, the proposed adaptive DSMC in 62 was not investigated under sampling and quantization imprecisions. Besides, regardless of the state equations of the physical model, it was assumed that an unknown multiplicative

\footnotetext{
${ }^{1}$ The material of this chapter has been published in the Journal of Control Engineering Practice [6] (doi:10.1016/j.conengprac.2016.10.017), and proceeding of the ASME 2016 Dynamic Systems and Control Conference [5] (doi:10.1115/DSCC2016-9732) with the permissions from Elsevier and ASME as shown in Appendix D
} 
term can represent the existing uncertainty in the model. In this chapter, in addition to the unknown multiplicative uncertainty term, a generic unknown additive term is considered in the model's equation to extend the adaptive DSMC formulation to a broader range of dynamical systems.

The contribution of this chapter is to extend the new DSMC with predicted implementation imprecisions from Chapter 4 to handle additive and multiplicative types of model uncertainties for linear and nonlinear systems using a discrete Lyapunov stability analysis. The proposed adaptive DSMC with predicted implementation imprecisions provides an integrated framework to improve the robustness of a common DSMC against both model and hardware (ADC) uncertainties. The performance of the proposed adaptive robust DSMC is evaluated on one linear (DC motor) and one nonlinear (automotive engine) models. Finally, the designed controllers are experimentally verified on a real ECU in real-time.

\subsection{Adaptation Against Model Uncertainties}

A general class of discrete SISO affine nonlinear systems can be described using the following state space representation:

$$
\begin{gathered}
x(i+1)=x(i)+T(f(x(t))+g(x(t)) u(t)) \\
y(i)=h(x(i))
\end{gathered}
$$


where $x \in \mathbb{R}^{r}$ is the state vector and $u \in \mathbb{R}$ and $h \in \mathbb{R}$ denote the system input and output, respectively. The uncertainties in the modeled dynamics $(f)$ can be expressed using unknown multiplicative $(\beta)$ and additive $(\alpha)$ terms in the presence of hardware (ADC) imprecisions $(\delta(i))$ using the nominal discrete nonlinear model in Eq. (5.1):

$$
x(i+1)=x(i)+T(\beta f+\alpha)+T g\left(u^{\bmod }(i)+\delta(i)\right)
$$

where $u^{\bmod }(i)$ is calculated according to the proposed approach in Chapter 4 from Eq. 4.19). Here, an adaptive DSMC theory is developed for handling the multiplicative and additive types of model uncertainties using Lyapunov stability theory. By applying the sliding reaching law $(s(i+1)<\rho s(i))$ for the discrete nonlinear system in Eq. (5.2) with uncertainty terms $(\alpha$ and $\beta$ ), the following relationship is concluded:

$$
s(i+1)=x(i)+T(\beta f+\alpha)+T g\left(u^{\bmod }(i)+\delta(i)\right)-x_{d}(i+1)
$$

where $\alpha$ and $\beta$ are unknown and constant. Modified control input $\left(u^{\bmod }\right)$ is calculated according to Eq. 4.22 in the presence of the uncertainty terms:

$$
u^{\bmod }(i)=\frac{1}{g T}\left[(\rho-1) x(i)-\rho x_{d}(i)-(\hat{\beta}(i) f+\hat{\alpha}(i)) T+x_{d}(i+1)\right]-\mu_{u}(i) s(i)
$$

where $\hat{\beta}(i)$ and $\hat{\alpha}(i)$ are estimations of the unknown multiplicative and additive parameters, respectively. We assume that by incorporating the predicted ADC function $\left(\mu_{u}(i) s(i)\right)$ into the DSMC structure, the ADC imprecisions on control signal $(\delta(i))$ are compensated. By substituting Eq. (5.4) into Eq. (5.3), the sliding surface dynamics 
can be simplified as follows:

$$
s(i+1)=\rho s(i)+T((\beta-\hat{\beta}(i)) f+(\alpha-\hat{\alpha}(i)))
$$

Two new terms ( $\tilde{\alpha}$ and $\tilde{\beta})$ are defined to represent the difference between the unknown and estimated uncertainty terms, $\tilde{\alpha}(i)=\alpha-\hat{\alpha}(i)$ and $\tilde{\beta}(i)=\beta-\hat{\beta}(i)$. The sliding surface dynamics, in terms of the DSMC gain $(\rho)$ and the errors in estimating the unknown parameters, can be obtained after substituting $\tilde{\alpha}$ and $\tilde{\beta}$ into Eq. 5.5):

$$
s(i+1)=\rho s(i)+T(\tilde{\beta}(i) f+\tilde{\alpha}(i))
$$

A Lyapunov-based analysis is employed to (i) determine the stability of the closedloop system, and (ii) derive the adaptation laws. The following Lyapunov function candidate is proposed:

$$
V(i)=\frac{1}{2} s^{2}(i)+\frac{1}{2} \rho_{\beta} \tilde{\beta}^{2}(i)+\frac{1}{2} \rho_{\alpha} \tilde{\alpha}^{2}(i)
$$

where $\rho_{\beta}>0$ and $\rho_{\alpha}>0$ are tunable adaptation gains that are chosen for the numerical sensitivity of the unknown multiplicative and additive parameter estimations, respectively. The proposed Lyapunov function is positive definite and quadratic with respect to the sliding variable $(s), \tilde{\beta}$, and $\tilde{\alpha}$. Similar to continuous-time systems, in which the negative definite condition is required for the derivative of the Lyapunov function to guarantee the asymptotic stability, in the discrete time domain, investigation of a Lyapunov difference function is required for the Lyapunov stability analysis. The Lyapunov difference function is calculated using an implicit approach in which 
the value of the Lyapunov function at the subsequent time step is obtained using a three-variable Taylor series expansion:

$$
\begin{gathered}
V(i+1)=V(i)+\frac{\partial V(i)}{\partial s(i)} \Delta s(i)+\frac{\partial V(i)}{\partial \tilde{\beta}(i)} \Delta \tilde{\beta}(i)+\frac{\partial V(i)}{\partial \tilde{\alpha}(i)} \Delta \tilde{\alpha}(i) \\
+\frac{1}{2} \frac{\partial^{2} V(i)}{\partial s^{2}(i)} \Delta s^{2}(i)+\frac{1}{2} \frac{\partial^{2} V(i)}{\partial \tilde{\beta}^{2}(i)} \Delta \tilde{\beta}^{2}(i)+\frac{1}{2} \frac{\partial^{2} V(i)}{\partial \tilde{\alpha}^{2}(i)} \Delta \tilde{\alpha}^{2}(i) \\
+\frac{\partial^{2} V(i)}{\partial s(i) \partial \tilde{\beta}(i)} \Delta s(i) \times \Delta \tilde{\beta}(i)+\frac{\partial^{2} V(i)}{\partial s(i) \partial \tilde{\alpha}(i)} \Delta s(i) \times \Delta \tilde{\alpha}(i) \\
+\frac{\partial^{2} V(i)}{\partial \tilde{\beta}(i) \partial \tilde{\alpha}(i)} \Delta \tilde{\beta}(i) \times \Delta \tilde{\alpha}(i)+\ldots
\end{gathered}
$$

where:

$$
\begin{gathered}
\Delta s(i) \equiv s(i+1)-s(i) \\
\Delta \tilde{\beta}(i) \equiv \tilde{\beta}(i+1)-\tilde{\beta}(i) \\
\Delta \tilde{\alpha}(i) \equiv \tilde{\alpha}(i+1)-\tilde{\alpha}(i)
\end{gathered}
$$

and the partial derivatives are as follows:

$$
\begin{gathered}
\frac{\partial V(i)}{\partial s(i)}=s(i), \quad \frac{\partial V(i)}{\partial \tilde{\beta}(i)}=\rho_{\beta} \tilde{\beta}(i), \quad \frac{\partial V(i)}{\partial \tilde{\alpha}(i)}=\rho_{\alpha} \tilde{\alpha}(i) \\
\frac{\partial^{2} V(i)}{\partial s^{2}(i)}=1, \quad \frac{\partial^{2} V(i)}{\partial \tilde{\beta}^{2}(i)}=\rho_{\beta}, \quad \frac{\partial^{2} V(i)}{\partial \tilde{\alpha}^{2}(i)}=\rho_{\alpha}, \\
\frac{\partial^{2} V(i)}{\partial s(i) \partial \tilde{\beta}(i)}=0, \quad \frac{\partial^{2} V(i)}{\partial s(i) \partial \tilde{\alpha}(i)}=0, \quad \frac{\partial^{2} V(i)}{\partial \tilde{\beta}(i) \partial \tilde{\alpha}(i)}=0
\end{gathered}
$$

Next, the Lyapunov difference function $(\Delta V(i)=V(i+1)-V(i))$ is calculated by substituting Eq. (5.9) into Eq. (5.2):

$$
\begin{gathered}
\Delta V(i)=s(i) \Delta s(i)+\rho_{\beta} \tilde{\beta}(i) \Delta \tilde{\beta}(i)+\rho_{\alpha} \tilde{\alpha}(i) \Delta \tilde{\alpha}(i) \\
+\frac{1}{2} \Delta s^{2}(i)+\frac{1}{2} \rho_{\beta} \Delta \tilde{\beta}^{2}(i)+\frac{1}{2} \rho_{\alpha} \Delta \tilde{\alpha}^{2}(i)+\ldots
\end{gathered}
$$


As can be observed from Eq. (5.9), all higher order $(>2)$ derivatives are zero. After substitution of Eq. (5.8) and (5.9) into Eq. (5.10), the Lyapunov difference function is simplified as follows:

$$
\begin{gathered}
\Delta V(i)=s(i)(s(i+1)-s(i)) \\
+\rho_{\beta} \tilde{\beta}(i)(\tilde{\beta}(i+1)-\tilde{\beta}(i))+\rho_{\alpha} \tilde{\alpha}(i)(\tilde{\alpha}(i+1)-\tilde{\alpha}(i)) \\
+\frac{1}{2} \Delta s^{2}(i)+\frac{1}{2} \rho_{\beta} \Delta \tilde{\beta}^{2}(i)+\frac{1}{2} \rho_{\alpha} \Delta \tilde{\alpha}^{2}(i)+\ldots
\end{gathered}
$$

which yields:

$$
\begin{gathered}
\Delta V(i)=(\rho-1) s^{2}(i)+T \tilde{\beta}(i) f s(i)+T \tilde{\alpha}(i) s(i)+ \\
+\rho_{\beta} \tilde{\beta}(i)(\tilde{\beta}(i+1)-\tilde{\beta}(i))+\rho_{\alpha} \tilde{\alpha}(i)(\tilde{\alpha}(i+1)-\tilde{\alpha}(i)) \\
+\frac{1}{2} \Delta s^{2}(i)+\frac{1}{2} \rho_{\beta} \Delta \tilde{\beta}^{2}(i)+\frac{1}{2} \rho_{\alpha} \Delta \tilde{\alpha}^{2}(i)+\ldots
\end{gathered}
$$

and finally:

$$
\begin{gathered}
\Delta V(i)=-(1-\rho) s^{2}(i) \\
+\rho_{\alpha} \tilde{\alpha}(i)\left(\tilde{\alpha}(i+1)-\tilde{\alpha}(i)+\frac{T s(i)}{\rho_{\alpha}}\right) \\
+\rho_{\beta} \tilde{\beta}(i)\left(\tilde{\beta}(i+1)-\tilde{\beta}(i)+\frac{T f s(i)}{\rho_{\beta}}\right) \\
+O\left(\Delta s^{2}(i), \Delta \tilde{\beta}^{2}(i), \Delta \tilde{\alpha}^{2}(i)\right)
\end{gathered}
$$

The following adaptation laws are chosen to update the errors in estimating the 
unknown multiplicative $(\tilde{\beta})$ and additive $(\tilde{\alpha})$ parameters:

$$
\begin{gathered}
\tilde{\beta}(i+1)=\tilde{\beta}(i)-\frac{T f s(i)}{\rho_{\beta}} \\
\tilde{\alpha}(i+1)=\tilde{\alpha}(i)-\frac{T s(i)}{\rho_{\alpha}}
\end{gathered}
$$

Upon incorporating the adaptation laws from Eq. (5.14) in the Lyapunov difference function, we have:

$$
\begin{gathered}
\Delta V(i) \approx-(1-\rho) s^{2}(i) \\
+\frac{1}{2}\left(\Delta s^{2}(i)+\rho_{\beta} \Delta \tilde{\beta}^{2}(i)+\rho_{\alpha} \Delta \tilde{\alpha}^{2}(i)\right)
\end{gathered}
$$

Next the second order terms in Eq. 5.15) are expanded and $\Delta \tilde{\beta}$ and $\Delta \tilde{\alpha}$ are replaced by the adaptation equations from Eq. (5.14):

$$
\begin{gathered}
\Delta V(i) \approx-(1-\rho) s^{2}(i) \\
+\frac{1}{2}\left((s(i+1)-s(i))^{2}+\rho_{\beta}\left(-\frac{T f s(i)}{\rho_{\beta}}\right)^{2}+\rho_{\alpha}\left(-\frac{T s(i)}{\rho_{\alpha}}\right)^{2}\right)
\end{gathered}
$$

For small enough $T$, all the terms in Eq. (5.16) with $T^{2}$ can be neglected. Additionally, $s(i+1)$ is replaced by $\rho s(i)$ :

$$
\Delta V(i) \approx\left((\rho-1)+\frac{1}{2}(\rho-1)^{2}\right) s^{2}(i)
$$

which yields:

$$
\Delta V(i) \approx \frac{1}{2}\left(\rho^{2}-1\right) s^{2}(i)
$$

Since $1>\rho>0$, Eq. 5.18 concludes that there exists a region around $s=0, \tilde{\beta}=0$ 
and $\tilde{\alpha}=0$ where the Lyapunov difference function is negative semi-definite. This means that the sliding variable (the tracking error, $s$ ) converges to zero, and the errors in estimating the unknown parameters $(\tilde{\beta}, \tilde{\alpha})$ are at least bounded.

Eq. (5.19) expresses the overall control input for the adaptive robust DSMC in which the ADC imprecisions are handled by incorporating the predicted uncertainties, and the multiplicative and additive unknown terms are updated according to the adaptation laws in Eq. (5.14):

$$
\begin{aligned}
& u_{\text {adaptive }}^{\text {mod }, n l}(i)= \\
& \frac{1}{g T}\left[(\rho-1) x(i)-\rho x_{d}(i)-(\hat{\beta} f+\hat{\alpha}) T+x_{d}(i+1)\right]-\mu_{u}(i) s(i)
\end{aligned}
$$

Figure 5.1 shows the schematic of the proposed adaptive robust DSMC for nonlin-

ear systems. In the next section, the new adaptive robust DSMC (Eq. (5.19) ) is re-formulated for linear systems, in which an analytic approach will be used for propagating the $\mathrm{ADC}$ imprecisions on control signals.

\subsection{Nonlinear Case Study: Engine Control}

The engine control system shown in Figure 5.2 is used to implement the adaptive DSMCs. As can be observed from Figure 5.2, $T_{e x h}, A F R\left(\dot{m}_{f}\right), m_{a}$, and $\omega_{e}$ can be measured on an engine; thus the ADC affects these four measured states and corresponding sliding surfaces. For analysis purposes, first a baseline adaptive SISO 


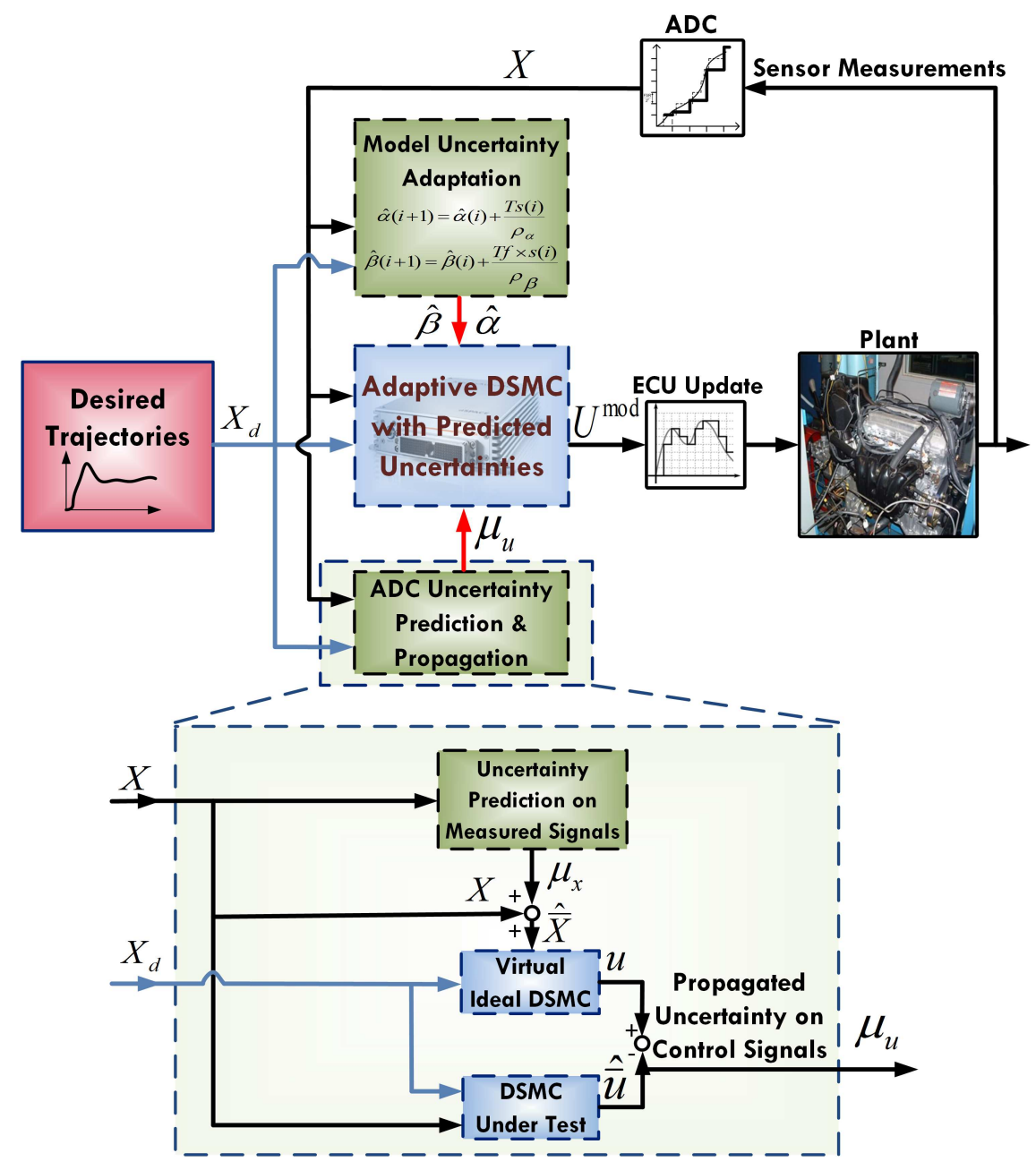

Figure 5.1: Schematic of the adaptive DSMC with online estimations of uncertainties on measured and control signals. $X_{d}$ is the desired trajectories (reference), $X$ is the measured signals from the plnat after ADC, $U_{\text {adaptive }}^{\text {mod }}$ is the control input, $\mu_{X}$ is the predicted ADC uncertainties on measured signals, $\mu_{U}$ is the propagated ADC uncertainties on control signals, and $\hat{\alpha}, \hat{\beta}$ are the estimated unknown parameters of the model.

DSMC (Eq. (5.4) and (5.14) ) is designed to drive the states of the system towards the desired values. Next, the predicted ADC uncertainties are incorporated into the adaptive DSMC formulation (Eq. (5.19)).

- Exhaust Gas Temperature Controller: According to Eq. (4.28), the function 


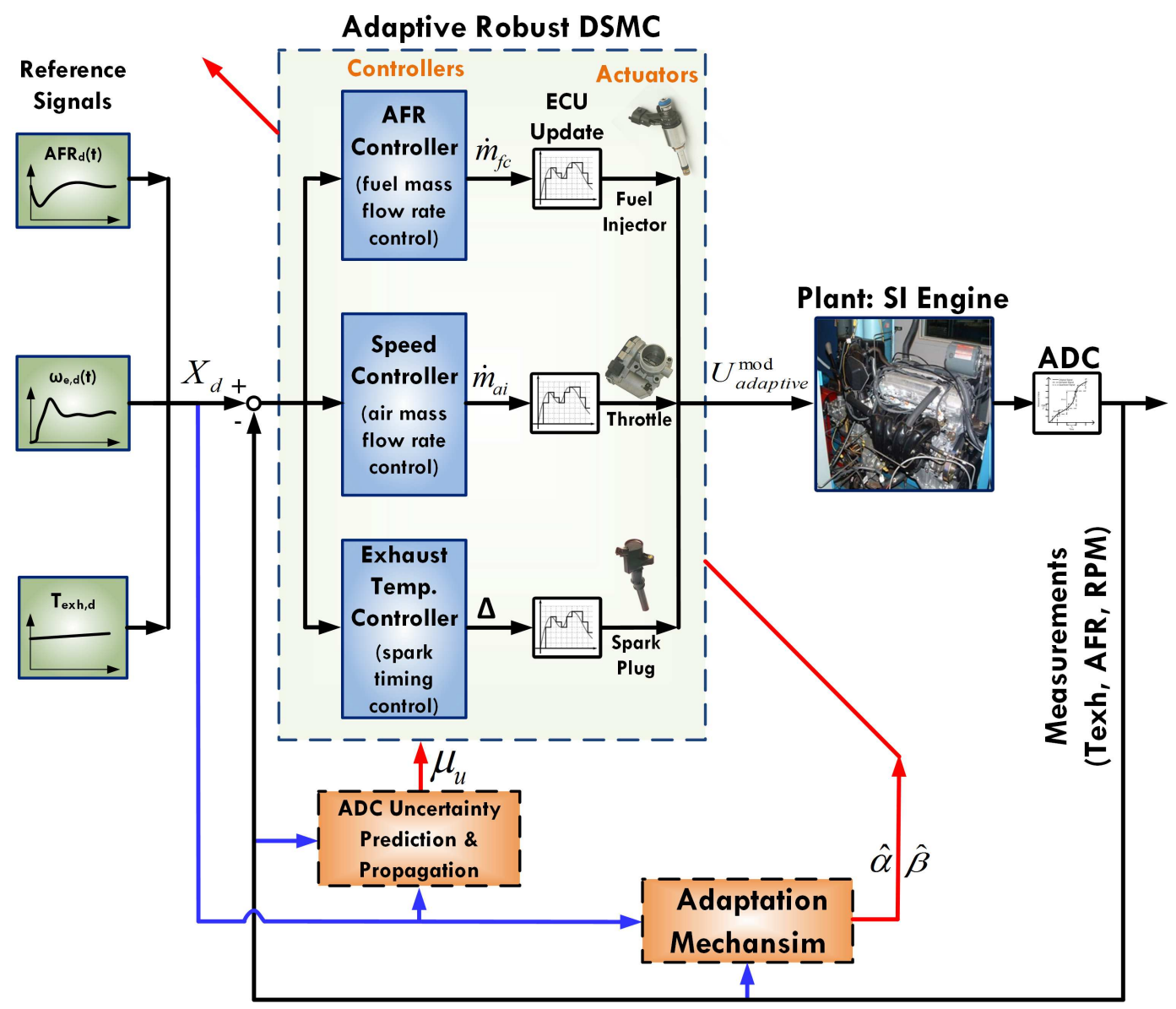

Figure 5.2: Block diagram of the engine cold start control system.

$f_{T_{e x h}}$ to capture dynamics of the exhaust gas temperature with additive $\left(\alpha_{T_{e x h}}\right)$ and multiplicative $\left(\beta_{T_{e x h}}\right)$ uncertainty terms is:

$$
f_{T_{e x h}}=\beta_{T_{e x h}} \times \frac{1}{\tau_{e}}\left(600 A F I-T_{e x h}\right)+\alpha_{T_{e x h}}
$$

Although in the empirical model of the exhaust gas temperature $\alpha_{T_{e x h}}$ has no physical meaning, it can present the error in the empirical model. The exhaust temperature dynamics depends heavily on the exhaust gas time constant $\left(\tau_{e}\right)$. Thus, any error 
in estimating the time constant $\left(\tau_{e}\right)$ results in significant deviation from the nominal model. To this end, the multiplicative uncertainty term $\left(\beta_{T_{e x h}}\right)$ is assumed to compensate for any error in estimating $\tau_{e}$. The errors in the modeled exhaust gas temperature dynamics are compensated according to the following adaptation laws with respect to Eq. (5.14):

$$
\begin{array}{r}
\hat{\beta}_{T_{e x h}}(i+1)=\hat{\beta}_{T_{e x h}}(i)+\frac{T\left(s_{1}(i)\right)}{\tau_{e} \rho_{\beta_{1}}}\left(600 A F I-T_{e x h}(i)\right) \\
\hat{\alpha}_{T_{e x h}}(i+1)=\hat{\alpha}_{T_{e x h}}(i)+\frac{T\left(T_{e x h}(i)-T_{e x h, d}(i)\right)}{\rho_{\alpha_{1}}}
\end{array}
$$

By incorporating the solutions of Eq. (5.21) and the predicted implementation imprecisions $\left(\mu_{\Delta}\right)$ into Eq. 5.19, the modified adaptive DSMC for exhaust temperature becomes:

$$
\begin{aligned}
\Delta(i)= & \frac{\tau_{e}}{7.5 A F I . T}\left[-\hat{\beta}_{T_{e x h}} \frac{T}{\tau_{e}}\left(600 A F I-T_{e x h}(i)\right)+\hat{\alpha}_{T_{e x h}}(i)\right. \\
& \left.+\left(\rho_{1}-1\right) s_{1}(i)+T_{e x h, d}(i+1)-T_{e x h, d}(i)\right]-\mu_{\Delta}(i) s_{1}(i)
\end{aligned}
$$

if $\mu_{\Delta}=0$ the controller is an adaptive baseline DSMC, while when $\mu_{\Delta}$ is calculated according to the mechanism in Figure 5.1, the controller is an integrated adaptive DSMC with predicted ADC uncertainties.

- Fuel Flow Rate Controller: As can be observed from Eq. 4.29, the fuel flow 
dynamics $\left(f_{\dot{m}_{f}}\right)$ with additive $\left(\alpha_{\dot{m}_{f}}\right)$ and multiplicative $\left(\beta_{\dot{m}_{f}}\right)$ uncertainty terms becomes:

$$
f_{\dot{m}_{f}}=-\beta_{\dot{m}_{f}} \times \frac{1}{\tau_{f}}\left(\dot{m}_{f}(i)\right)+\alpha_{\dot{m}_{f}}
$$

In practice, $\dot{m}_{f}$ is not measured directly and it is calculated according to the AFR sensor measurement and estimated air mass inside the cylinder $\left(\dot{m}_{a o}\right) . \alpha_{\dot{m}_{f}}$ represents the error in estimating fuel flow rate due to AFR measurement uncertainty and error in predicting the air mass. Similar to exhaust gas temperature dynamics, the fuel evaporation time constant $\tau_{f}$ dictates the dynamics of the fuel flow into the cylinder. Thus, any error in estimating the time constant $\left(\tau_{f}\right)$ results in significant deviation from the nominal model. To this end, the multiplicative uncertainty is introduced to the fuel flow dynamics to represent the uncertainty in estimating $\tau_{f}$. The adaptation laws for $\beta_{\dot{m}_{f}}$ and $\alpha_{\dot{m}_{f}}$ are:

$$
\begin{array}{r}
\hat{\beta}_{\dot{m}_{f}}(i+1)=\hat{\beta}_{\dot{m}_{f}}(i)-\frac{T\left(s_{2}(i)\right)}{\tau_{f} \rho_{\beta_{2}}} \dot{m}_{f}(i) \\
\hat{\alpha}_{\dot{m}_{f}}(i+1)=\hat{\alpha}_{\dot{m}_{f}}(i)+\frac{T\left(\dot{m}_{f}(i)-\dot{m}_{f, d}(i)\right)}{\rho_{\alpha_{2}}}
\end{array}
$$

where $\dot{m}_{f, d}$ is calculated according to the desired AFR. The adaptive control law for fuel flow rate into the cylinder with multiplicative and additive unknown terms and the predicted ADC imprecisions $\left(\mu_{\dot{m}_{f c}}\right)$ is:

$$
\begin{array}{r}
\dot{m}_{f c}(i)=\frac{\tau_{f}}{T}\left[\hat{\beta}_{\dot{m}_{f}} \frac{T}{\tau_{f}}\left(\dot{m}_{f}(i)\right)+\hat{\alpha}_{\dot{m}_{f}}(i)+\left(\rho_{2}-1\right) s_{2}(i)\right. \\
\left.+\dot{m}_{f, d}(i+1)-\dot{m}_{f, d}(i)\right]-\mu_{\dot{m}_{f c}}(i) s_{2}(i)
\end{array}
$$


- Engine Speed Controller: $f_{\omega_{e}}$ for the engine (Eq. 4.30) with multiplicative $\left(\beta_{\omega_{e}}\right)$ and additive $\left(\alpha_{\omega_{e}}\right)$ unknown parameters is:

$$
f_{\omega_{e}}=-\beta_{\omega_{e}} \times \frac{1}{J}\left(T_{\text {loss }}\right)+\alpha_{\omega_{e}}
$$

where $T_{\text {loss }}=0.4 \omega_{e}+100 . T_{\text {loss }}$ represents the torque losses (e.g., due to friction) on the crankshaft. Thus, the additive uncertainty $\alpha_{\omega_{e}}$ represents the error in reading the torque map. Since reading the values from the torque map between break points in the look-up table uses a linear relationship, the additive uncertainty term can express the potential error in the modeled dynamics accordingly. Also $\beta_{\omega_{e}}$ represents the uncertainty in estimating the effective engine inertia $(J) . \beta_{\omega_{e}}$ and $\alpha_{\omega_{e}}$ are driven to zero using the following adaptation laws:

$$
\begin{array}{r}
\hat{\beta}_{\omega_{e}}(i+1)=\hat{\beta}_{\omega_{e}}(i)-\frac{T\left(s_{3}(i)\right)}{J \rho_{\beta_{3}}}\left(0.4 \omega_{e}(i)+100\right) \\
\hat{\alpha}_{\omega_{e}}(i+1)=\hat{\alpha}_{\omega_{e}}(i)+\frac{T\left(\omega_{e}(i)-\omega_{e, d}(i)\right)}{\rho_{\alpha_{3}}}
\end{array}
$$

Finally, the control input $\left(m_{a, d}\right)$ for engine speed regulation after incorporating the propagated ADC uncertainties $\left(\mu_{m_{a, d}}\right)$ yields:

$$
\begin{gathered}
m_{a, d}(i)=\frac{J}{30,000 T}\left[\hat{\beta}_{\omega_{e}} \frac{T}{J}\left(100+0.4 \omega_{e}(i)\right)+\hat{\alpha}_{\omega_{e}}(i)\right. \\
\left.+\left(\rho_{3}-1\right) s_{3}(i)+\omega_{e, d}(i+1)-\omega_{e, d}(i)\right]-\mu_{m_{a, d}}(i) s_{3}(i)
\end{gathered}
$$

- Air Mass Flow Controller: The intake air manifold dynamics are linked to the rotational dynamics through the calculated $m_{a, d}$ (Eq. 4.31). The calculated $m_{a, d}$ 
from Eq. 5.28 is used as the desired trajectory to obtain $\dot{m}_{a i}$ as the control input of the intake air flow rate controller. The intake manifold air mass dynamics with additive model uncertainty $\left(\alpha_{m_{a}}\right)$ and the multiplicative unknown parameter $\left(\beta_{m_{a}}\right)$ is:

$$
f_{m_{a}}=-\beta_{m_{a}}\left(\dot{m}_{a o}(i)\right)+\alpha_{m_{a}}
$$

where [84]:

$$
\begin{gathered}
\dot{m}_{a o}=k_{1} \eta_{v o l} m_{a} \omega_{e} \\
\eta_{v o l}=m_{a}^{2}\left(k_{2} \omega_{e}^{2}+k_{3} \omega_{e}+k_{4}\right)+m_{a}\left(k_{5} \omega_{e}^{2}+k_{6} \omega_{e}+k_{7}\right) \\
+k_{8} \omega_{e}^{2}+k_{9} \omega_{e}+k_{10}
\end{gathered}
$$

$k_{1,2, \ldots, 10}$ are the empirical parameters of the volumetric efficiency $\left(\eta_{v o l}\right)$ curve fit. As shown in Eq. (5.29)-(5.31), the uncertainty terms in the intake air manifold dynamics $\left(\alpha_{m_{a}}\right.$ and $\beta_{m_{a}}$ ) compensate for the uncertainties in $\dot{m}_{a o}$ which is read through the $\eta_{v o l}$ curve fit. $\beta_{m_{a}}$ and $\alpha_{m_{a}}$ are updated using the following adaptation laws:

$$
\begin{array}{r}
\hat{\beta}_{m_{a}}(i+1)=\hat{\beta}_{m_{a}}(i)-\frac{T\left(s_{4}(i)\right)}{\rho_{\beta_{4}}} \dot{m}_{a o} \\
\hat{\alpha}_{m_{a}}(i+1)=\hat{\alpha}_{m_{a}}(i)+\frac{T\left(m_{a}(i)-m_{a, d}(i)\right)}{\rho_{\alpha_{4}}}
\end{array}
$$

After incorporating the predicted ADC imprecisions $\left(\mu_{\dot{m}_{a i}}\right)$ into the air mass controller, the adaptive robust controller input is:

$$
\begin{aligned}
& \dot{m}_{a i}(i)=\frac{1}{T}\left[T \hat{\beta}_{m_{a}}\left(\dot{m}_{a o}(i)\right)+\hat{\alpha}_{m_{a}}(i)+\left(\rho_{4}-1\right) s_{4}(i)\right. \\
& \left.+m_{a, d}(i+1)-m_{a, d}(i)\right]-\mu_{\dot{m}_{a i}}(i) s_{4}(i)
\end{aligned}
$$


In Eq. 5.22, 5.25, 5.28), and 5.33, $\mu_{\Delta}, \mu_{\dot{m}_{f c}}, \mu_{m_{a, d}}$, and $\mu_{\dot{m}_{a i}}$ are the estimations of propagated ADC uncertainties on control signals which are computed according to the approach explained in Chapter 4 and Figure 5.1. Estimation of propagated ADC uncertainties on control signals requires the knowledge of ADC uncertainty on measured signals $\left(\mu_{T_{e x h}}, \mu_{\dot{m}_{f}}, \mu_{\omega_{e}}\right.$, and $\left.\mu_{m_{a}}\right)$ that are predicted using Eq. (4.6). The verification results of the estimated ADC uncertainties of the engine plant are summarized in Figure 5.3 and Table 5.1 for a set of measured $T_{e x h}, \dot{m}_{f}, \omega_{e}$, and $m_{a}$ signals under $10 \mathrm{~ms}$ of sampling time and quantization level of 10-bit. For better evaluation of the uncertainty prediction mechanism, the measured engine signals are defined in their worst and nonsmooth shapes. The high accuracy of the ADC uncertainty prediction mechanism can be observed for the engine case study from Figure 5.3 and Table 5.1 .

Table 5.1

Mean $(\bar{e})$ and standard deviation $\left(\sigma_{e}\right)$ of ADC uncertainty prediction errors for the SI engine model.

\begin{tabular}{lcc}
\hline \hline & $\bar{e}$ & $\sigma_{e}$ \\
\hline$T_{\text {exh }}\left[{ }^{\circ} \mathrm{C}\right]$ & 0.5 & 0.5 \\
$\dot{m}_{f}[\mathrm{~kg} / \mathrm{sec}]$ & $2.337 \times 10^{-6}$ & $2.732 \times 10^{-6}$ \\
$\omega_{e}[\mathrm{rad} / \mathrm{sec}]$ & 0.124 & 0.145 \\
$m_{a}[\mathrm{~kg}]$ & $2.060 \times 10^{-5}$ & $2.321 \times 10^{-5}$ \\
\hline \hline
\end{tabular}

The impact of unknown additive and multiplicative terms on part of the baseline engine DSMC that is associated with the plant's dynamics $(f)$ is shown in Figure 5.4 . The multiplicative terms $(\beta)$ for the engine case study were assumed to be \pm 0.75 , 


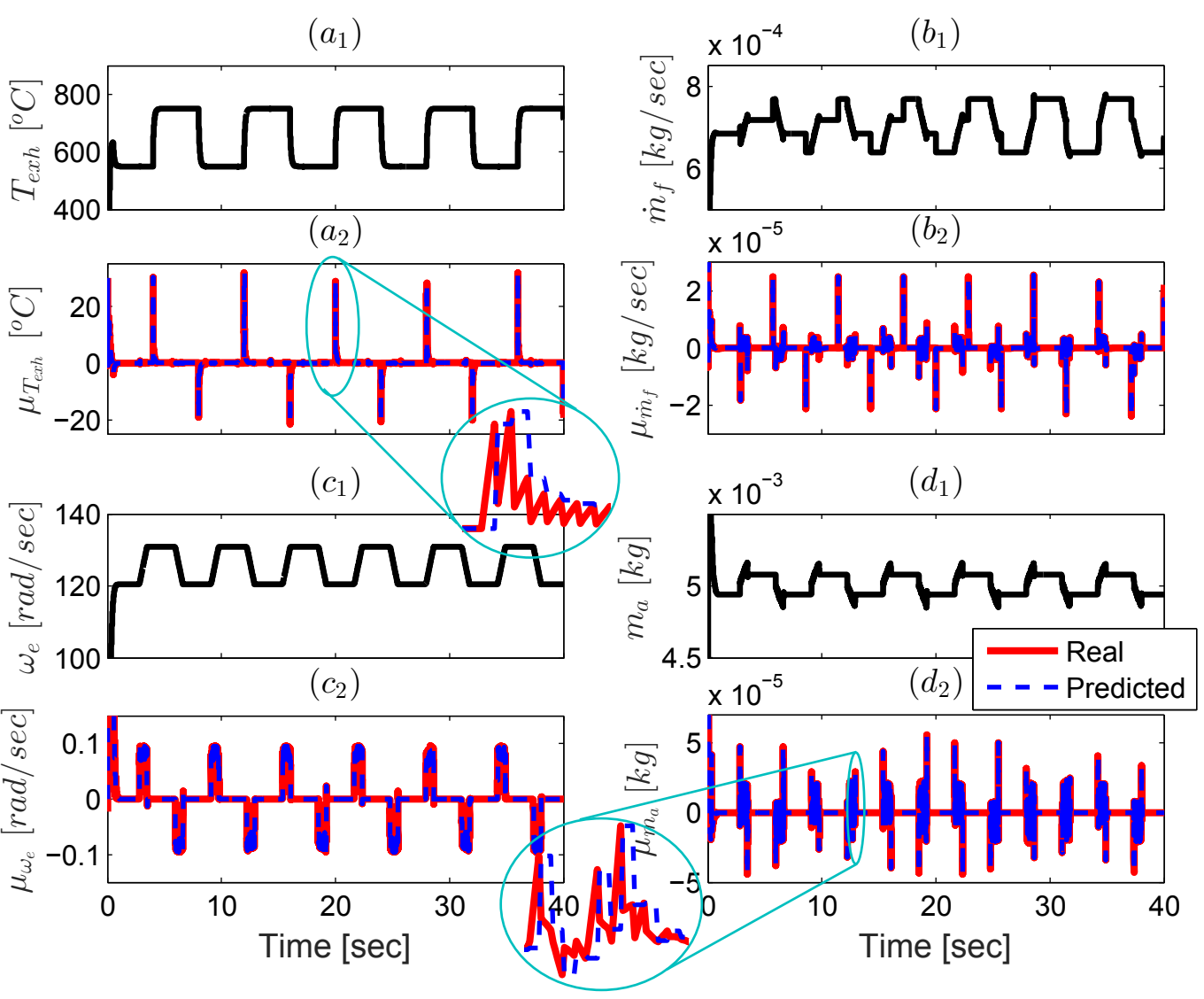

Figure 5.3: Comparison of actual and predicted uncertainties due to sampling and quantization on measured variables of the SI engine: (a) $T_{\text {exh }}$ (b) $\dot{m}_{f}$, (c) $\omega_{e}$, and (d) $m_{a}$ (10 ms sampling time and 10-bit quantization level).

which means up to $25 \%$ error on each of the states dynamics. The equivalent additive unknown terms $(\alpha)$, for which the dynamics show a similar deviation from the nominal model due to corresponding multiplicative unknown terms, were found for every single dynamic. In the absence of the adaptation, the selected values for the additive unknown terms result in the same errors in the dynamics which were already observed from the multiplicative terms. 
(a)

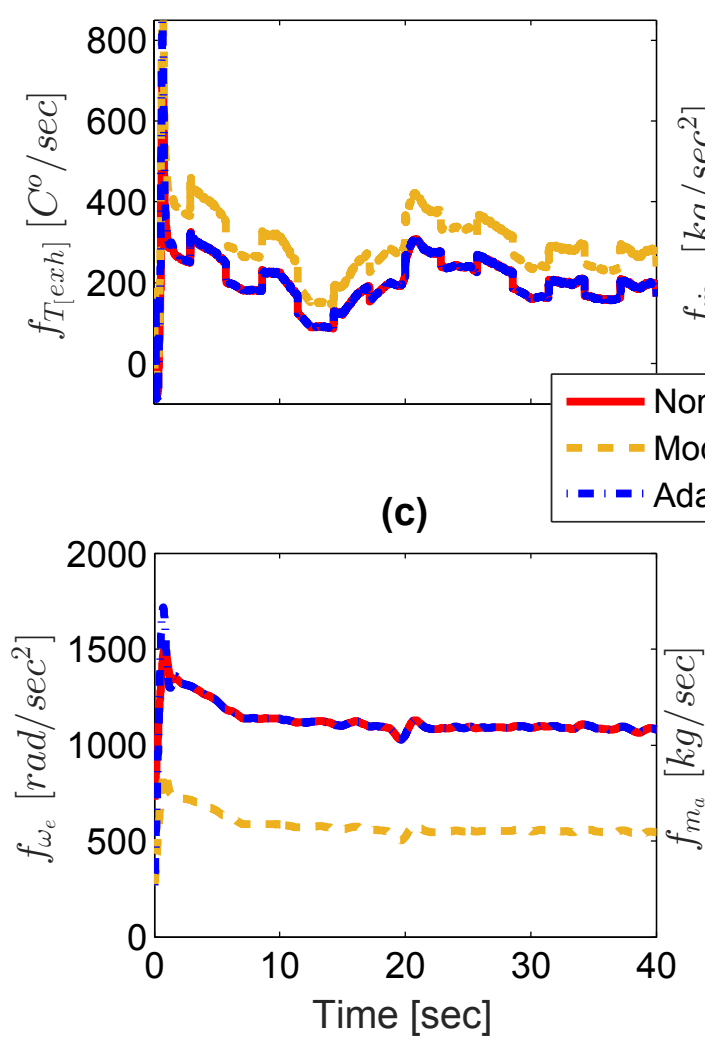

(b)

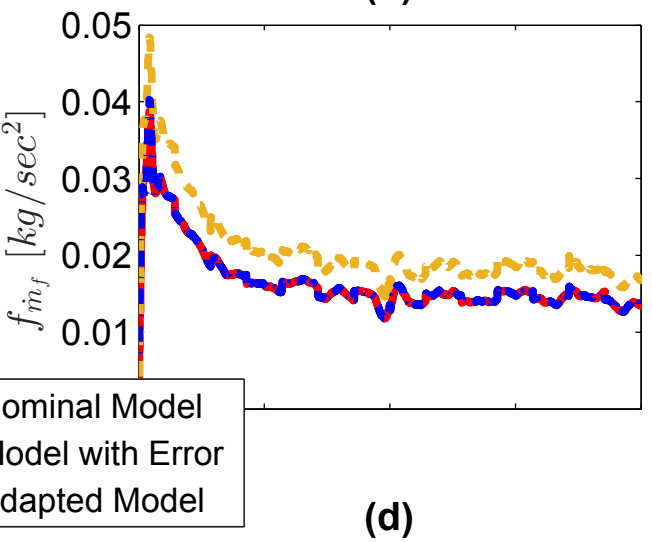

Figure 5.4: The impacts of unknown uncertainty terms on the engine dynamics inside the DSMC and how the adaptation mechanisms drive the model with error to its nominal value: (a) $T_{e x h}$, (b) $\dot{m}_{f}$, (c) $\omega_{e}$, and (d) $m_{a}$ (10 ms sampling time and 10-bit quantization level).

The uncertainties on the model parameters cause a permanent error in the estimated dynamics compared to the nominal model (model with no uncertainty). These errors significantly demolish the DSMC tracking performance and consequently, the outcome of the non-adaptive DSMC will not be acceptable anymore. Upon activation of the adaptation mechanisms (Eq. (5.14), it can be seen from Figure 5.4 that the model with error converges to the nominal model and the model uncertainties are compensated. Figure 5.5 shows the results of unknown multiplicative and additive 

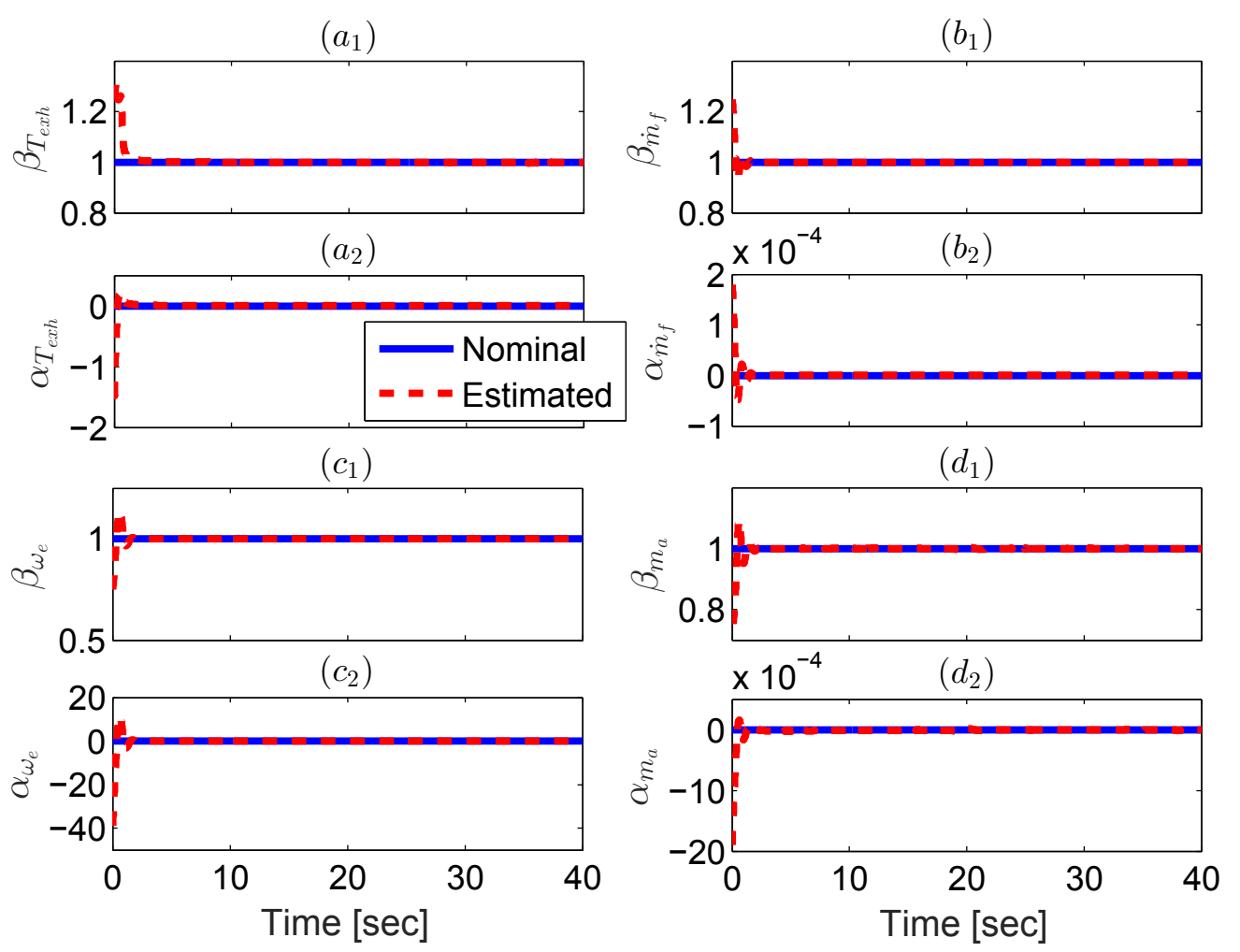

Figure 5.5: Convergence results of unknown engine model multiplicative and additive terms (10 $\mathrm{ms}$ sampling time and 10-bit quantization level).

uncertainty terms estimation against the actual (nominal) values. Despite the short time period (40 sec) during which the engine cold start controller should operate, the adaptation mechanism removes the errors in the modeled dynamics in the DSMC in less than $2 \mathrm{sec}$ by driving the unknown multiplicative and additive parameters to their nominal values, "1" and "0", respectively.

Figure 5.6 shows the impact of errors in the engine's model on the DSMC tracking performance. As it is expected, the non-adaptive DMSC fails to track the desired trajectories, which explains the importance of handling the model uncertainties in the 
body of the proposed DSMC. On the other hand, once the adaptation algorithms are activated and the convergence period of the unknown parameters is over, the baseline adaptive DSMC tracks all the desired trajectories smoothly with the minimum error under $10 \mathrm{~ms}$ of sampling time and quantization level of 10-bit.

(a)

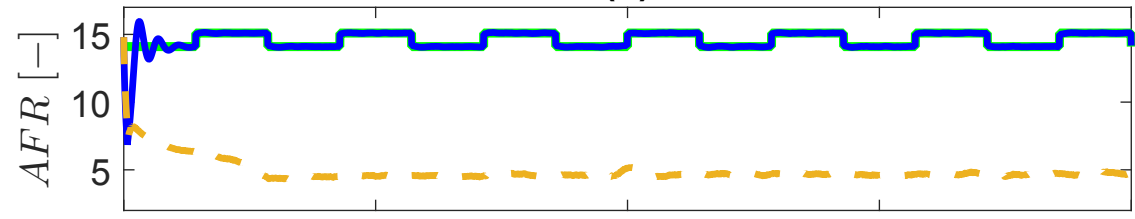

(b)
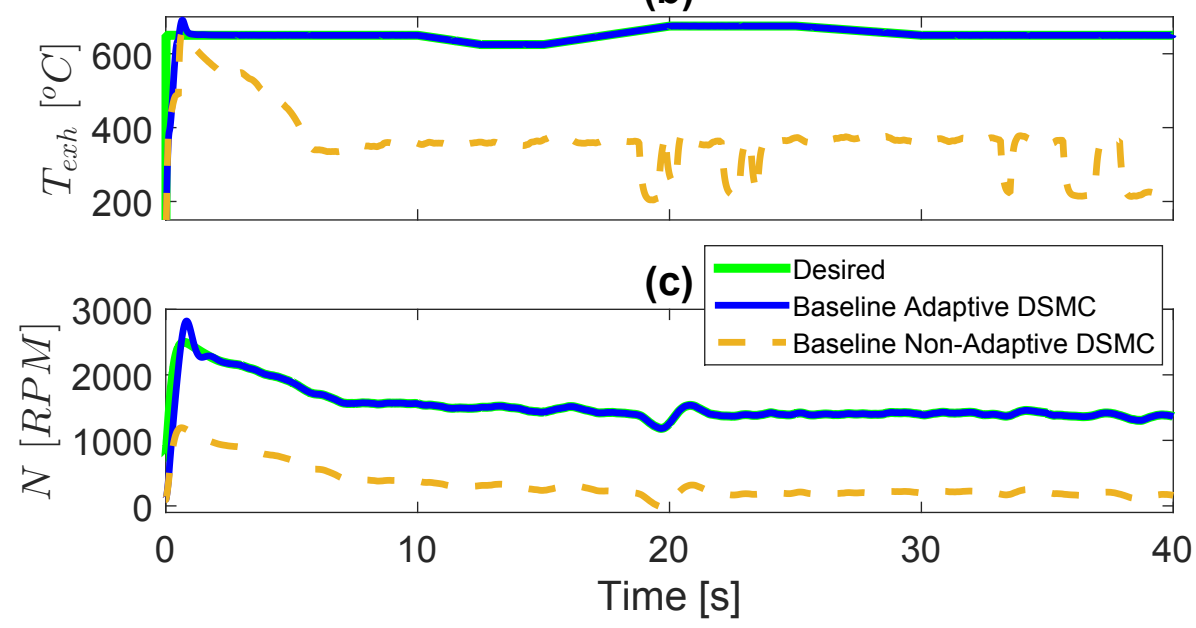

Figure 5.6: Comparison between baseline adaptive and non-adaptive engine DSMCs under ADC imprecisions and model uncertainties: (a) air-fuel ratio, (b) exhaust gas temperature, and (c) engine speed (10 $\mathrm{ms}$ sampling time and 10-bit quantization level).

Table 5.2 summarizes the effect of unknown parameter variations (25\% error from each type of model uncertainties) on the tracking error (Table 5.2 -a), and dynamic responses (Table 5.2-b) for baseline non-adaptive and adaptive DSMCs. By comparing the errors in trajectory tracking and dynamic estimation of the non-adaptive DSMC with those from the adaptive DSMC, one can conclude the effectiveness of 
the proposed adaptation mechanism in handling the model uncertainties for all cases.

It can be observed that the adaptive DSMC enhances the trajectory tracking errors by more than $95 \%$ compared to the non-adaptive DSMC. The better tracking performance of the adaptive controller is a direct result of the accurate and fast estimation of the unknown parameters by the adaptation technique. The adaptation mechanism removes the errors in the engine dynamics by up to $98 \%$.

\section{Table 5.2}

Mean $(\bar{e})$ value of the errors in (a) trajectory tracking (Figure 5.6), and (b) estimating the engine dynamics (Figure 5.4), from the non-adaptive and adaptive DSMCs under $25 \%$ uncertainty in each of the multiplicative $(\beta)$ and additive $(\alpha)$ unknown terms in the engine dynamics (Values inside the parentheses show the resulting improvement from the robust DSMC compared to the baseline DSMC, $T=10 \mathrm{~ms}$, quantization level=10-bit).

(a)

\begin{tabular}{|c|c|c|c|c|c|}
\hline & \multicolumn{2}{|c|}{$\bar{e}$ (Tracking Error) } & & \multicolumn{2}{|c|}{$\bar{e}$ (Model Error) } \\
\hline & \multirow{3}{*}{$\begin{array}{c}\text { Non-adaptive } \\
\text { DSMC } \\
\text { Reference }\end{array}$} & \multirow{3}{*}{$\begin{array}{c}\text { Adaptive } \\
\text { DSMC }\end{array}$} & & Non-adaptive & Adaptive \\
\hline & & & & DSMC & DSMC \\
\hline & & & $f_{\dot{m}_{f}}$ & 0.0042 & 0.000086 \\
\hline \multirow{2}{*}{$\begin{array}{l}\text { AFR } \\
{[-]}\end{array}$} & \multirow[t]{2}{*}{9.566} & \multirow{2}{*}{$\begin{array}{c}0.103 \\
(-98.92 \%) \\
\end{array}$} & {$\left[\mathrm{kg} / \mathrm{sec}^{2}\right]$} & & $(-97.95 \%)$ \\
\hline & & & $f_{T_{e x h}}$ & 87.1 & 1.8 \\
\hline \multirow{2}{*}{$\begin{array}{l}T_{e x h} \\
{\left[{ }^{\circ} C\right]}\end{array}$} & \multirow[t]{2}{*}{292.2} & \multirow{2}{*}{$\begin{array}{c}3.6 \\
(-98.8 \%) \\
\end{array}$} & {$\left[{ }^{\circ} \mathrm{C} / \mathrm{sec}\right]$} & & $(-97.8 \%)$ \\
\hline & & & & 547.0 & 7.6 \\
\hline \multirow{2}{*}{$\begin{array}{l}N \\
{[R P M]}\end{array}$} & \multirow[t]{2}{*}{1202.9} & \multirow{2}{*}{$\begin{array}{c}17.6 \\
(-98.5 \%)\end{array}$} & {$\left[\mathrm{rad} / \mathrm{sec}^{2}\right]$} & & $(-98.6 \%)$ \\
\hline & & & & 0.0055 & 0.000082 \\
\hline
\end{tabular}

In the next stage, the predicted implementation imprecisions are incorporated in the adaptive DSMC to compare the performance of the baseline adaptive controller versus the adaptive DSMC with predicted ADC uncertainties. Figure 5.7 shows the overall DSMC performance in tracking the desired $A F R$, exhaust gas temperature, and engine speed trajectories in the presence of unknown uncertainty terms and under 
(a)
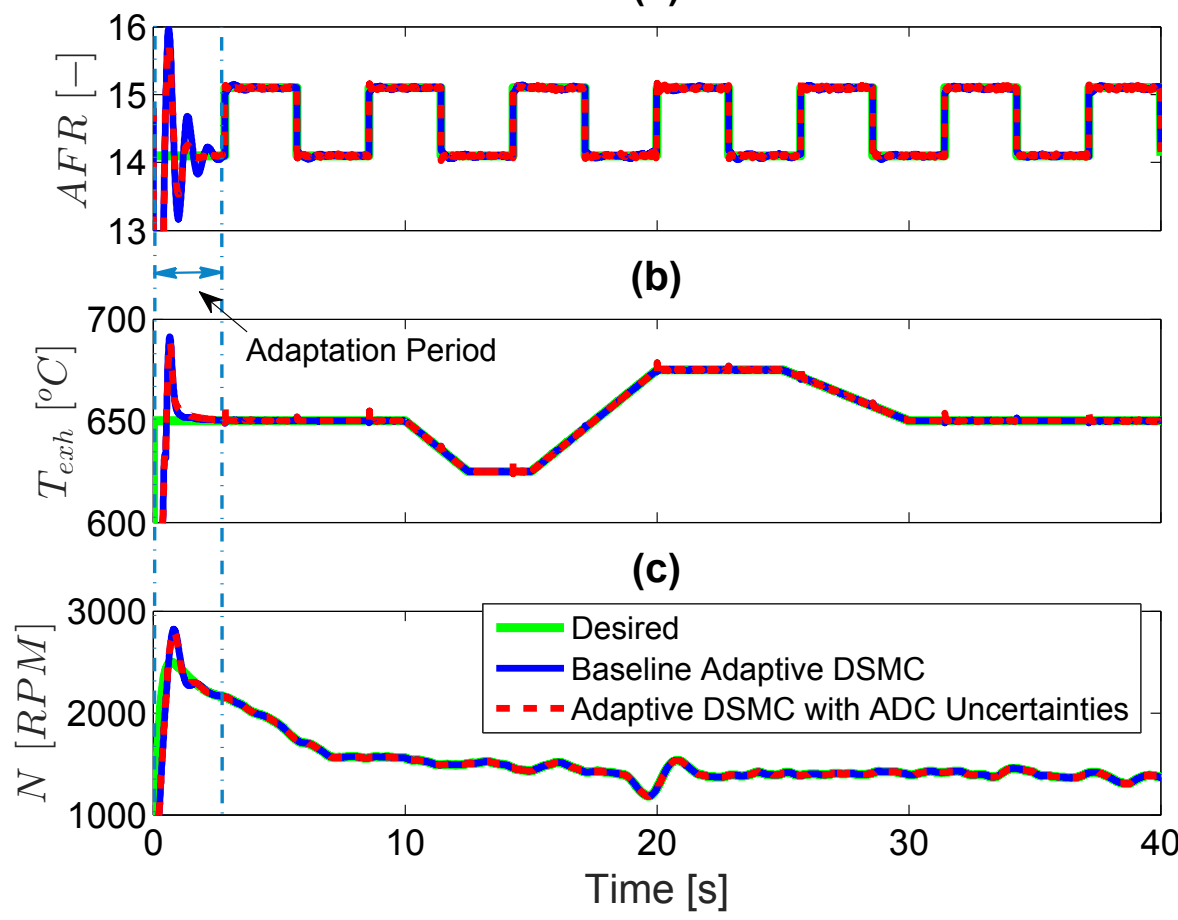

Figure 5.7: Results of engine control under ADC imprecisions and model uncertainties: (a) air-fuel ratio, (b) exhaust gas temperature, and (c) engine speed (10 ms sampling time and 10-bit quantization level).

$10 \mathrm{~ms}$ of sampling time and quantization level of 10-bit. It can be observed from Figure 5.7 that after the adaptation mechanisms removed the model uncertainties within $t=2 \mathrm{sec}$, the DSMC tracks all the desired trajectories desirably. Figure 5.7 shows that ADC with sampling time of $10 \mathrm{~ms}$ and quantization level of 10-bit does not affect the baseline DSMC performance significantly. However, as can be observed from Figure 5.8, once the sampling time increases, or when the quantization level becomes smaller, the tracking errors from the baseline adaptive DSMC increase dramatically. On the other side, the proposed adaptive DSMC with incorporated ADC imprecisions is more robust to the sampling time increase and also the quantization level decrease. 
$\left(a_{1}\right)$

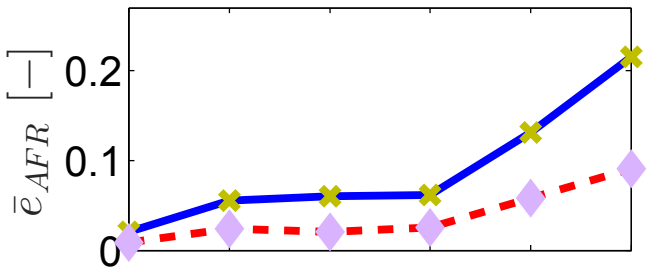

$\left(a_{2}\right)$

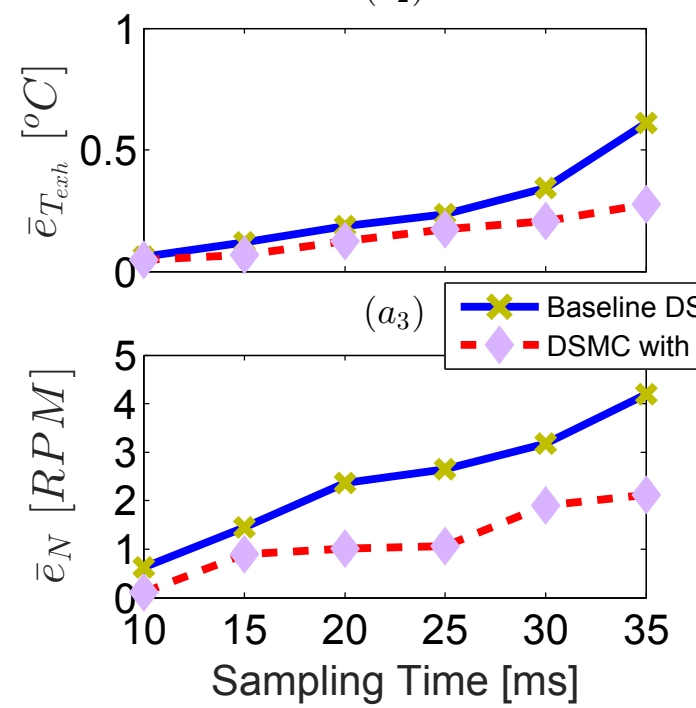

$\left(b_{1}\right)$

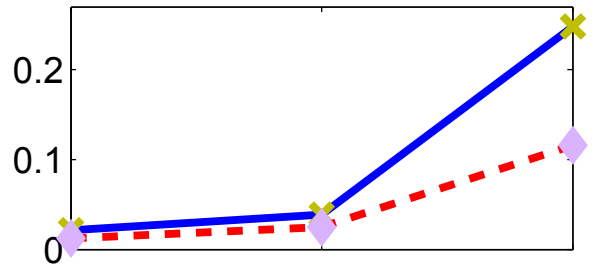

$\left(b_{2}\right)$

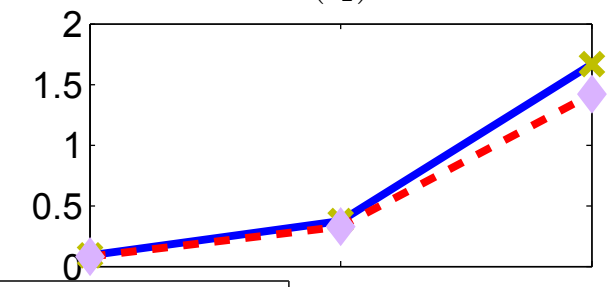

$\left(b_{3}\right)$

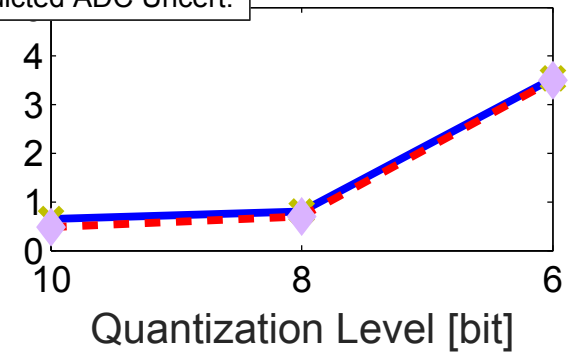

Figure 5.8: Effect of sampling time and quantization level on the tracking performance of the engine controller with and without the predicted ADC uncertainties.

$A F R$ is the most important trajectory due to its effects on engine performance in meeting the desired emission and fuel consumption targets [3]. It can be seen from Figure 5.8 that when the signals at the controller I/O are sampled at a higher rate than $10 \mathrm{~ms}$, the baseline adaptive DSMC shows weak performance in tracking the desired AFR trajectory. The desired AFR tracking error rises by more than 6 times when the sampling time increases from $10 \mathrm{~ms}$ to $35 \mathrm{~ms}$. Meanwhile, the adaptive DSMC with predicted ADC uncertainties keeps the tracking error less than 0.1 , despite the increase in the sampling time. This means an overall $50 \%$ decrease in AFR 
tracking error compared to the baseline adaptive DSMC. Similar improvement trends can be concluded from $T_{e x h}$ and $\omega_{e}$ adaptive DSMCs in which the tracking errors of the baseline controllers at higher sampling times are decreased by 50-60\% upon incorporating the predicted ADC uncertainties.

Figure 5.9 provides an overall comparison among the baseline non-adaptive DSMC, baseline adaptive DSMC, and robust adaptive DSMC with predicted ADC uncertainties. When there is no uncertainty in the model, it can be observed that by incorporating the predicted ADC uncertainties, comparing to the baseline controller, the controller becomes more robust against sampling and quantization (sampling time of $10 \mathrm{~ms}$ and quantization level of 10-bit) imprecisions by more than $50 \%$ on average in terms of the mean tracking errors (Figure $5.9-a_{1}, b_{1}, c_{1}$ ). By introducing uncertainties to the engine model, both adaptive and robust adaptive DSMCs show significantly better performances, compared to the baseline DSMC (Figure 5.9 $a_{2}, b_{2}, c_{2}$ ). Moreover, in the presence of model and ADC uncertainties, the robust adaptive DSMC shows lower errors by 50-60\%, compared to the baseline adaptive DSMC for all cases (Figure 5.9. $a_{3}, b_{3}, c_{3}$ ).

The required computational time for the engine adaptive DSMC was also studied for various controller configurations using the MATLAB/Simulink MIL platform. The MIL testing was carried out on a desktop computer with an Intel Core ${ }^{T M}$ i5 @ 3.20 $\mathrm{GHz}$ processor. It was observed that the average computational time of the baseline 


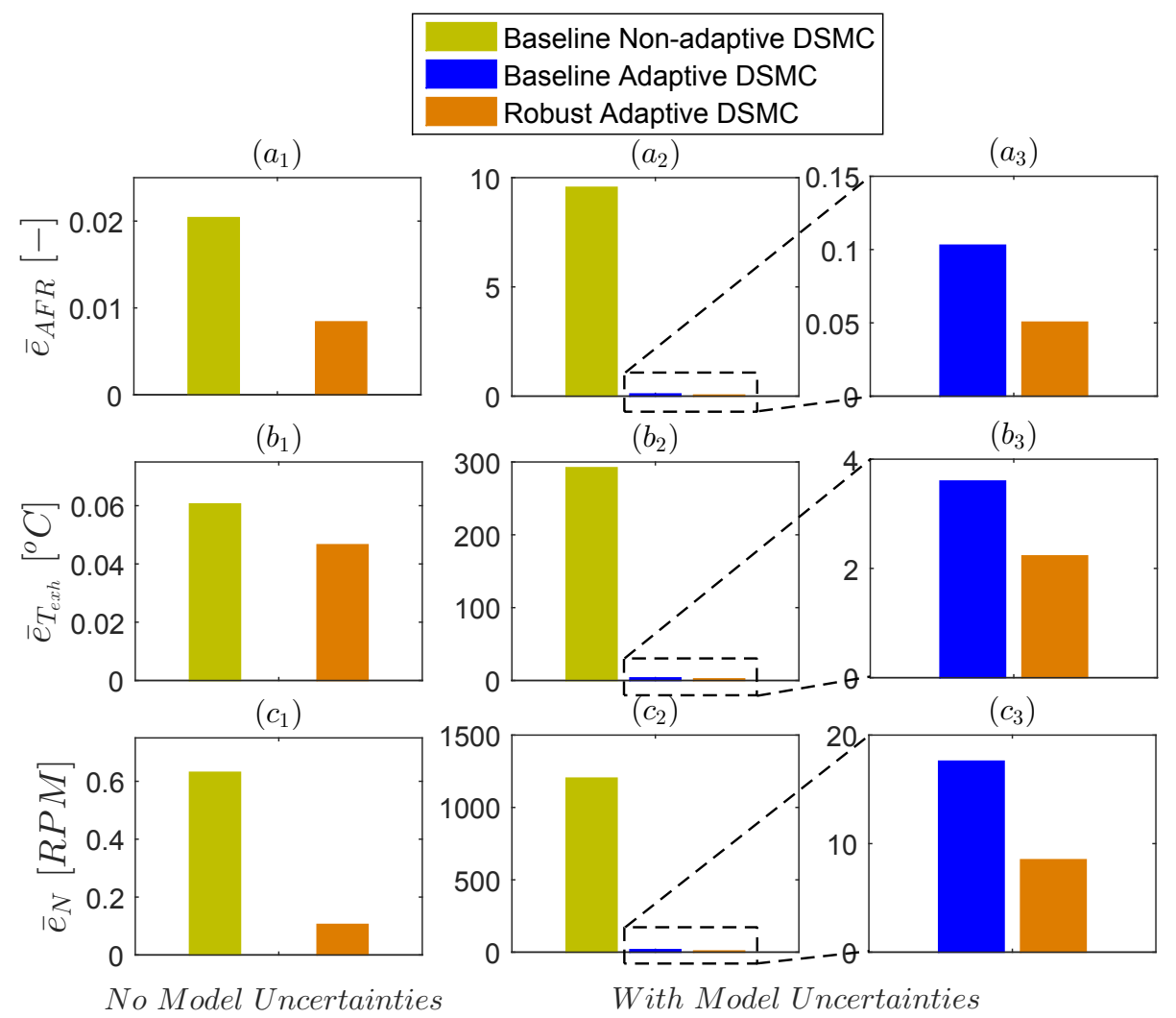

Figure 5.9: Effects of incorporating the predicted ADC uncertainties and the adaptation laws on the DSMC robustness characteristics under $25 \%$ uncertainty in each of the multiplicative $(\beta)$ and additive $(\alpha)$ unknown terms, and $10 \mathrm{~ms}$ sampling time and 10-bit quantization level.

DSMC for controlling the engine during the first $40 \mathrm{sec}$ of its operation is $3.012 \mathrm{sec}$.

These computational times for the adaptive DSMCs when (i) both of the adaptation laws are active, (ii) only the multiplicative adaptation law is active, and (iii) only the additive adaptation law is active, are $3.062 \mathrm{sec}, 3.036 \mathrm{sec}$, and $3.041 \mathrm{sec}$, respectively. The computational time of the adaptive DSMCs are slightly higher than the baseline DSMC $(<2 \%)$ and this shows the effectiveness of the proposed adaptation algorithm that allows for real-time implementation.

In the engine model, the time constants in the fuel mass flow $\left(\tau_{f}\right)$ and exhaust gas 
temperature $\left(\tau_{e}\right)$ dynamics take into account empirical time delays within the physical engine dynamics. However, the time delay in the hardware and associated uncertainty are shown in the literature that can be addressed through SMC design [91, 92, 93]. Since most of the recent works in the literature are limited to linear or continuous time systems, extending the proposed adaptive robust DSMC design to nonlinear time delayed systems needs to be investigated in future studies.

\subsection{First Order Adaptive DSMC for Linear Un- certain Systems}

In this section, the proposed DSMC with predicted ADC imprecisions for linear systems from Section 4.2 .2 in Chapter 4 is extended to linear uncertain systems under modeling uncertainties. In the absence of model uncertainties and implementation imprecisions, the state-space equation of a $r^{t h}$-order linear system, discretized by using a first order Euler approximation [88, is represented by:

$$
\mathbf{X}_{r \times 1}(i+1)=\left(T \mathbf{A}_{r \times r}+\mathbf{I}_{r \times r}\right) \mathbf{X}_{r \times 1}(i)+T \mathbf{B}_{r \times h} \mathbf{U}_{h \times 1}
$$

where $x \subset \mathbf{X} \in \mathbb{R}^{r}, u \subset \mathbf{U} \in \mathbb{R}^{h}$, and $\mathbf{I} \in \mathbb{R}^{r \times r}$ are the state vector, the control input vector, and identity matrix, respectively. Matrix A represents the linear system dynamics, and its elements, $a_{p q}, p, q=1, \ldots, r$ are obtained based on the system's physical equations and the interaction among different states. In practice, the identified matrix A is subjected to several sources of uncertainties, e.g. the plant parameter variations over time. 
The linear system in Eq. (5.34), in the presence of additive $(\alpha)$ and multiplicative $(\beta)$ types of modeling uncertainties, and ADC imprecisions, can be presented as follows:

$$
\begin{aligned}
& \mathbf{X}_{r \times 1}(i+1)= \\
& T\left[\begin{array}{cccc}
\beta_{11} a_{11}+\alpha_{11} & \beta_{12} a_{12}+\alpha_{12} & \cdots & \beta_{1 r} a_{1 r}+\alpha_{1 r} \\
\beta_{21} a_{21}+\alpha_{21} & \beta_{22} a_{22}+\alpha_{22} & \cdots & \beta_{2 r} a_{2 r}+\alpha_{2 r} \\
\vdots & \vdots & \ddots & \vdots \\
\beta_{r 1} a_{r 1}+\alpha_{r 1} & \beta_{r 2} a_{r 2}+\alpha_{r 2} & \cdots & \beta_{r r} a_{r r}+\alpha_{r r}
\end{array}\right] \\
& +\left[\begin{array}{cccc}
1 & 0 & \cdots & 0 \\
0 & 1 & \cdots & 0 \\
\vdots & \vdots & \ddots & \vdots \\
0 & 0 & \cdots & 1
\end{array}\right]_{r \times r}\left[\begin{array}{c}
x_{1}(i) \\
x_{2}(i) \\
\vdots \\
x_{r}(i)
\end{array}\right]+T \mathbf{B}_{r \times r}\left(\mathbf{U}_{r \times 1}+\delta_{r \times 1}(i)\right)
\end{aligned}
$$

where $a_{p q}$ represents nominal values of the ideal linear system's dynamics $(\mathbf{A})$, and $\delta_{r \times 1}$ is the vector of propagated ADC uncertainties on the control signal (U). As can be seen, for each element of $\mathbf{A}$, one additive $(\alpha)$ and one multiplicative $(\beta)$ unknown terms are considered to present any errors or variation in the values of the model parameters. For the uncertain linear system in Eq. (5.35) under implementation imprecisions, the tracking control problem is defined to drive the states of the system $(x)$ to their desired values $\left(x_{d} \subset \mathbf{X}_{d} \in \mathbb{R}^{r}\right)$. To this end, SISO/MIMO first order DSMC, under modelling and implementation uncertainties, are formulated in the following sections.

For the linear system in Eq. (5.34), it was shown in Section 4.2 .2 of Chapter 4 that 
the first order DSMC input $\mathbf{U}(i)$ is obtained according to the following first order sliding reaching law [4, 89]:

$$
|\mathbf{S}(i+1)| \leq \mathcal{P}|\mathbf{S}(i)|
$$

where, the vector $(\mathbf{S})$ of the first order sliding surface variables $(s)$ is defined as the difference between the desired $\left(x_{d}\right)$ and the measured signal $(x)$ as follows:

$$
\mathbf{S}(i)=\mathbf{X}(i)-\mathbf{X}_{d}(i)
$$

and, $\mathcal{P}$ is the matrix of tunable DSMC gains. For a SISO DSMC, $\mathcal{P}$ is diagonal: $\mathcal{P}=$ $\operatorname{diag}\left[\rho_{1}, \ldots, \rho_{r}\right]$, where $0<\rho_{1, \ldots, r}<1$ [89]. On the other hand, for a MIMO DSMC structure, the off-diagonal elements of $\mathcal{P}$ can be non-zero; however, the eigenvalues of $\mathcal{P}$ should lie within the unit circle to guarantee the closed-loop system stability [62]. If $r=h, \mathbf{B}$ is a square matrix, and the control input vector $\mathbf{U}$ can be calculated as follows for the linear system according to the sliding reaching law from Eq. (5.36):

$$
\mathbf{U}(i)=\mathbf{B}^{-1}\left(\frac{1}{T}\left[(\mathcal{P}-\mathbf{I}) \mathbf{X}(i)-\mathcal{P} \mathbf{X}_{d}(i)+\mathbf{X}_{d}(i+1)\right]-\mathbf{A X}(i)\right)
$$

For the linear systems, as discussed in Chapter 4 , the propagated ADC imprecision on the control signals is the difference between $\mathbf{U}$ and $\overline{\mathbf{U}}$, where $\mathbf{U}$ is calculated according to Eq. (5.38). $\overline{\mathbf{U}}$ is the control input of an ideal first order DSMC where there is neither model uncertainties nor ADC imprecisions. $\overline{\mathbf{U}}(i)-\mathbf{U}(i)$ can be analytically found with respect to Eq. 4.14): 


$$
\overline{\mathbf{U}}(i)-\mathbf{U}(i)=\mathbf{B}^{-1}\left(\frac{1}{T}\left[(\mathcal{P}-\mathbf{I}) \mu_{X}(i)\right]-\mathbf{A} \mu_{X}(i)\right)
$$

A new diagonal matrix $\left(\mu_{\mathbf{U}}\right)$ is defined with respect to the estimated propagated ADC uncertainties on the control signals:

$$
\mu_{\mathbf{U}}=\operatorname{diag}\left[\bar{u}_{1}-u_{1}, \ldots, \bar{u}_{r}-u_{r}\right]
$$

where $u \subset \mathbf{U}$ and $\bar{u} \subset \overline{\mathbf{U}}$. The diagonal elements of $\mu_{\mathbf{U}}$ are calculated with respect to Eq. (5.39). The propagated ADC imprecisions on the control signals are shown in Eq. (5.35) by $\delta$. Here, similar to the proposed approach for nonlinear systems, the approach to overcome the ADC uncertainties is inclusion of the propagated ADC uncertainties on control signals $\left(\mu_{\mathbf{U}}\right)$ into the DSMC structure. For the linear $(l)$ system (Eq. (5.38) ), in the absence of model uncertainties, the control input (U) of the conventional first order DSMC is modified against data sampling and quantization imprecisions as follows:

$$
\begin{gathered}
\mathbf{U}^{m o d, l}(i)=\mathbf{B}^{-1}\left(\frac{1}{T}\left\{(\mathcal{P}-\mathbf{I}) \mathbf{X}(i)-\mathcal{P} \mathbf{X}_{d}(i)+\mathbf{X}_{d}(i+1)\right\}-\mathbf{A X}(i)\right) \\
-\left|\mu_{\mathbf{U}}(i)\right| \times(\mathbf{S}(i))
\end{gathered}
$$

where $\mu_{\mathbf{U}}$ is calculated according to Eq. (5.40).

In the next step, the uncertainties in the model are included in the first order DSMC formulation. It can be easily shown that the first order sliding vector $(\mathbf{S})$ for the linear system with unknown additive and multiplicative parameters becomes: 


$$
\begin{aligned}
& \mathbf{S}_{r \times 1}(i+1)=\mathcal{P}_{r \times r} \mathbf{S}_{r \times 1}(i)+ \\
& T\left[\begin{array}{cccc}
\tilde{\beta}_{11} a_{11}+\tilde{\alpha}_{11} & \tilde{\beta}_{12} a_{12}+\tilde{\alpha}_{12} & \cdots & \tilde{\beta}_{1 r} a_{1 r}+\tilde{\alpha}_{1 r} \\
\tilde{\beta}_{21} a_{21}+\tilde{\alpha}_{21} & \tilde{\beta}_{22} a_{22}+\tilde{\alpha}_{22} & \cdots & \tilde{\beta}_{2 r} a_{2 r}+\tilde{\alpha}_{2 r} \\
\vdots & \vdots & \ddots & \vdots \\
\tilde{\beta}_{r 1} a_{r 1}+\tilde{\alpha}_{r 1} & \tilde{\beta}_{r 2} a_{r 2}+\tilde{\alpha}_{r 2} & \cdots & \tilde{\beta}_{r r} a_{r r}+\tilde{\alpha}_{r r}
\end{array}\right] \mathbf{X}_{r \times 1}(i)
\end{aligned}
$$

where $\tilde{\beta}_{p q}=\beta_{p q}-\hat{\beta}_{p q}$ and $\tilde{\alpha}_{p q}=\alpha_{p q}-\hat{\alpha}_{p q}$ are the errors in estimating the unknown multiplicative and additive parameters, respectively.

- Theorem: The adaptation laws for converging the unknown parameters of the uncertain linear system (Eq. 5.35) ) to their nominal values $(\hat{\beta} \rightarrow \beta, \hat{\alpha} \rightarrow \alpha)$, based on the DSMC formulation, are as follows:

$$
\begin{array}{r}
\hat{\beta}_{p q}(i+1)=\hat{\beta}_{p q}(i)+\frac{T s_{p}(i) a_{p q} x_{q}(i)}{\rho_{\beta_{p q}}} \\
\hat{\alpha}_{p q}(i+1)=\hat{\alpha}_{p q}(i)+\frac{T s_{p}(i) x_{q}(i)}{\rho_{\alpha_{p q}}}
\end{array}
$$

where, $p, q=1 \ldots r$, and $\rho_{\beta}$ and $\rho_{\alpha}$ are tunable positive multiplicative and additive adaptation gains chosen for the numerical sensitivity in the estimations of the unknown parameters.

Proof: A Lyapunov stability analysis is performed to derive the adaptation laws and guarantee the stability of the closed-loop system. To this end, the analysis begins with a first-order linear uncertain system $(r=1)$. The following positive definite scalar Lyapunov function $(\mathbf{V})$ for a first order linear system is proposed: 


$$
V(i)=\frac{1}{2} s^{2}(i)+\frac{1}{2} \rho_{\beta} \tilde{\beta}^{2}(i)+\frac{1}{2} \rho_{\alpha} \tilde{\alpha}^{2}(i)
$$

As can be seen, $\mathbf{V}$ is a quadratic function of the tracking error $(\mathbf{S})$, and unknown parameters estimations. In the next step, the first order Lyapunov difference function $(\Delta V)$ is calculated. $\Delta V$ is obtained by applying a Taylor series expansion on Eq. (5.44):

$$
\begin{gathered}
\Delta V(i)=\frac{\partial V(i)}{\partial s(i)} \Delta s(i)+\frac{\partial V(i)}{\partial \tilde{\beta}(i)} \Delta \tilde{\beta}(i)+\frac{\partial V(i)}{\partial \tilde{\alpha}(i)} \Delta \tilde{\alpha}(i)+ \\
\frac{1}{2} \frac{\partial^{2} V(i)}{\partial s^{2}(i)} \Delta s^{2}(i)+\frac{1}{2} \frac{\partial^{2} V(i)}{\partial \tilde{\beta}^{2}(i)} \Delta \tilde{\beta}^{2}(i)+\frac{1}{2} \frac{\partial^{2} V(i)}{\partial \tilde{\alpha}^{2}(i)} \Delta \tilde{\alpha}^{2}(i)+\ldots
\end{gathered}
$$

where, $\Delta s(i) \equiv s(i+1)-s(i), \Delta \tilde{\beta}(i) \equiv \tilde{\beta}(i+1)-\tilde{\beta}(i), \Delta \tilde{\alpha}(i) \equiv \tilde{\alpha}(i+1)-\tilde{\alpha}(i)$. Upon substitution of partial derivatives into Eq. (5.45), we have:

$$
\begin{gathered}
\Delta V(i)=s(i) \Delta s(i)+\rho_{\beta} \tilde{\beta}(i) \Delta \tilde{\beta}(i)+\rho_{\alpha} \tilde{\alpha}(i) \Delta \tilde{\alpha}(i)+ \\
\frac{1}{2} \Delta s^{2}(i)+\frac{1}{2} \rho_{\beta} \Delta \tilde{\beta}^{2}(i)+\frac{1}{2} \rho_{\alpha} \Delta \tilde{\alpha}^{2}(i)+\ldots
\end{gathered}
$$

It is assumed that for small enough sampling periods, $\Delta s^{2}(i), \Delta \tilde{\beta}^{2}(i), \Delta \tilde{\alpha}^{2}(i) \approx 0[1]$. The same assumption is valid for higher order terms also (>2). By using Eq. (5.42) for the first order system, Eq. (5.46) can be simplified as follows:

$$
\begin{gathered}
\Delta V(i)=s(i)((\rho-1) s(i)+T(\tilde{\beta}(i) a+\tilde{\alpha}(i)) x(i))+ \\
\rho_{\beta} \tilde{\beta}(i) \Delta \tilde{\beta}(i)+\rho_{\alpha} \tilde{\alpha}(i) \Delta \tilde{\alpha}(i)
\end{gathered}
$$

Eq. (5.47) can be re-arranged as follows: 


$$
\begin{gathered}
\Delta V(i)=(\rho-1) s^{2}(i)+\rho_{\beta} \tilde{\beta}(i)\left(\frac{T \cdot s(i) \cdot a \cdot x(i)}{\rho_{\beta}}+\Delta \tilde{\beta}(i)\right) \\
+\rho_{\alpha} \tilde{\alpha}(i)\left(\frac{T . s(i) \cdot x(i)}{\rho_{\alpha}}+\Delta \tilde{\alpha}(i)\right)
\end{gathered}
$$

If $\hat{\beta}$ and $\hat{\alpha}$ are updated according to the following rules:

$$
\begin{array}{r}
\hat{\beta}(i+1)=\hat{\beta}(i)+\frac{T \cdot s(i) \cdot a \cdot x(i)}{\rho_{\beta}} \\
\hat{\alpha}(i+1)=\hat{\alpha}(i)+\frac{T \cdot s(i) \cdot x(i)}{\rho_{\alpha}}
\end{array}
$$

then, Eq. (5.48) becomes:

$$
\Delta V(i)=-(1-\rho) s^{2}(i)
$$

As can be seen, since $0<\rho<1$, Eq. 5.50 is negative semi-definite. This means that the positive definite Lyapunov function $V$ has a negative semi-definite difference function $(\Delta V)$. Thus, according to the Lyapunov stability theorem and the new Invariance Principle for discontinuous systems [1, 57, 59, 94], the asymptotic stability of the closed loop controller with the adaptation laws in Eq. (5.49) is guaranteed. This ensures the finite time convergences of the first order sliding function $(s)$ and the unknown parameter estimation errors $(\tilde{\beta}, \tilde{\alpha})$ to zero.

The performed Lyapunov stability can be extended to higher order systems. For a second order system $(r=2)$, two scalar positive definite Lyapunov functions $\left(V_{1}, V_{2}\right)$ can be defined for each of the system's states. If one can show that both Lyapunov functions have negative semi-definite difference functions, the overall stability of the second order linear system can be concluded. For a SISO first order DSMC, the sliding 
function of the first state $\left(s_{1}\right)$ can be obtained as follows according to Eq. 5.42):

$$
\begin{gathered}
s_{1}(i+1)=\rho_{1} s_{1}(i)+ \\
T\left(\left(\tilde{\beta}_{11} a_{11}+\tilde{\alpha}_{11}\right) x_{1}(i)+\left(\tilde{\beta}_{12} a_{12}+\tilde{\alpha}_{12}\right) x_{2}(i)\right)
\end{gathered}
$$

For $s_{1}$, the following positive definite Lyapunov function is introduced:

$$
\begin{gathered}
V_{1}(i)=\frac{1}{2} s_{1}^{2}(i)+\frac{1}{2} \rho_{\beta_{11}} \tilde{\beta}_{11}^{2}(i)+\frac{1}{2} \rho_{\beta_{12}} \tilde{\beta}_{12}^{2}(i)+ \\
\frac{1}{2} \rho_{\alpha_{11}} \tilde{\alpha}_{11}^{2}(i)+\frac{1}{2} \rho_{\alpha_{12}} \tilde{\alpha}_{12}^{2}(i)
\end{gathered}
$$

Similar to the first order linear system stability analysis, it can be easily shown that the Lyapunov difference function $\left(\Delta V_{1}\right)$ becomes:

$$
\begin{gathered}
\Delta V_{1}(i)=\left(\rho_{1}-1\right) s_{1}^{2}(i)+ \\
\rho_{\beta_{11}} \tilde{\beta}_{11}(i)\left(\frac{T \cdot s_{1}(i) \cdot a_{11} \cdot x_{1}(i)}{\rho_{\beta_{11}}}+\Delta \tilde{\beta}_{11}(i)\right)+ \\
\rho_{\alpha_{11}} \tilde{\alpha}_{11}(i)\left(\frac{T \cdot s_{1}(i) \cdot x_{1}(i)}{\rho_{\alpha_{11}}}+\Delta \tilde{\alpha}_{11}(i)\right)+ \\
\rho_{\beta_{12}} \tilde{\beta}_{12}(i)\left(\frac{T \cdot s_{1}(i) \cdot a_{12} \cdot x_{2}(i)}{\rho_{\beta_{12}}}+\Delta \tilde{\beta}_{12}(i)\right)+ \\
\rho_{\alpha_{12}} \tilde{\alpha}_{12}(i)\left(\frac{T \cdot s_{1}(i) \cdot x_{2}(i)}{\rho_{\alpha_{12}}}+\Delta \tilde{\alpha}_{12}(i)\right)
\end{gathered}
$$

As can be seen, if the adaptation laws from Eq. (5.43) are used to update $\tilde{\beta}_{11}, \tilde{\beta}_{12}$, $\tilde{\alpha}_{11}$, and $\tilde{\alpha}_{12}$ in Eq. (5.53), the Lyapunov difference function becomes $\Delta V_{1}(i)=$ $-\left(1-\rho_{1}\right) s_{1}^{2}(i)$, which fulfills the required negative semi-definite condition for $\Delta V_{1}$ and guarantees the finite-time zero convergence of $s_{1}, \tilde{\beta}_{11}, \tilde{\beta}_{12}, \tilde{\alpha}_{11}$, and $\tilde{\alpha}_{12}$. The same conclusions can be reached for higher order systems $(r>2)$ by utilizing the adaptation laws in Eq. (5.43) to update the unknown multiplicative and additive 
parameters within the linear system dynamics.

Overall, the control input of an adaptive first order DSMC with incorporated implementation imprecisions and adaptation laws from Eq. (5.43) becomes:

$$
\begin{gathered}
\mathbf{U}_{\text {adaptive }}^{\text {mod },}(i)=\frac{\mathbf{B}^{-1}}{T}\left(\mathcal{P}_{r \times r} \mathbf{S}_{r \times 1}(i)+\mathbf{X}_{d}(i+1)\right. \\
\left.-\left(T \hat{\mathbf{A}}+\mathbf{I}_{r \times r}\right) \mathbf{X}(i)\right)-\left|\mu_{\mathbf{U}}\right| \times \operatorname{sat}\left(\mathbf{S}_{r \times 1}(i)\right)
\end{gathered}
$$

where:

$$
\hat{\mathbf{A}}=\left[\begin{array}{cccc}
\hat{\beta}_{11} a_{11}+\hat{\alpha}_{11} & \hat{\beta}_{12} a_{12}+\hat{\alpha}_{12} & \cdots & \hat{\beta}_{1 r} a_{1 r}+\hat{\alpha}_{1 r} \\
\hat{\beta}_{21} a_{21}+\hat{\alpha}_{21} & \hat{\beta}_{22} a_{22}+\hat{\alpha}_{22} & \cdots & \hat{\beta}_{2 r} a_{2 r}+\hat{\alpha}_{2 r} \\
\vdots & \vdots & \ddots & \vdots \\
\hat{\beta}_{r 1} a_{r 1}+\hat{\alpha}_{r 1} & \hat{\beta}_{r 2} a_{r 2}+\hat{\alpha}_{r 2} & \cdots & \hat{\beta}_{r r} a_{r r}+\hat{\alpha}_{r r}
\end{array}\right]
$$

\subsubsection{Linear Case Study: DC Motor Speed Control}

The state equations of the DC motor model introduced in Section 4.3.1 (Eq. 4.23) with unknown multiplicative $(\beta)$ and additive $(\alpha)$ terms become:

$$
\begin{gathered}
\theta(i+1)=T\left(\left[-\beta_{11} \frac{k_{f}}{J}+\alpha_{11}\right] \theta(i)+\left[\alpha_{12}\right] \mathcal{I}(i)\right)+T\left(\frac{k_{m}}{J} \mathcal{I} v(i)+\frac{1}{J} \Gamma\right)+\theta(i) \\
\mathcal{I}(i+1)=T\left(\left[-\beta_{21} \frac{k_{b}}{L}+\alpha_{21}\right] \theta(i)+\left[-\beta_{22} \frac{R}{L}+\alpha_{22}\right] \mathcal{I}(i)\right)+\frac{T}{L} V(i)+\mathcal{I}(i)
\end{gathered}
$$

Four additive $\left(\alpha_{p q}\right)$ and three multiplicative $\left(\beta_{p q}\right)$ unknown parameters are derived to their nominal values by solving the adaptation laws in Eq. (5.43). The final adaptive first order SISO DSMC with predicted ADC uncertainties yields: 


$$
\begin{array}{r}
\mathcal{I}_{\text {d,adaptive }}^{\text {mod }}(i)=\frac{J}{k_{m}}\left(\frac{1}{T}\left[\rho_{1}\left(s_{1}(i)\right)+\theta_{d}(i+1)-\theta(i)\right]+\right. \\
\left.\left[\hat{\beta}_{11} \frac{k_{f}}{J}+\hat{\alpha}_{11}\right] \theta(i)+\left[\hat{\alpha}_{21}\right] \mathcal{I}(i)-\frac{1}{J} \Gamma\right)-\left|\mu_{\mathcal{I}_{d}}(i)\right| \operatorname{sat}\left(s_{1}(i)\right) \\
V_{\text {adaptive }}^{\text {mod }}(i)=L\left(\frac{1}{T}\left[\rho_{2}\left(s_{2}(i)\right)+\mathcal{I}_{d}(i+1)-\mathcal{I}(i)\right]\right. \\
\left.+\left[\hat{\beta}_{21} \frac{k_{b}}{L}+\hat{\alpha}_{21}\right] \theta(i)+\left[\hat{\beta}_{22} \frac{R}{L}+\hat{\alpha}_{22}\right] \mathcal{I}(i)\right)-\left|\mu_{V}(i)\right| \operatorname{sat}\left(s_{2}(i)\right)
\end{array}
$$

The convergence results of the unknown parameters are shown in Figure 5.10. This is done by simultaneously solving Eq. (5.43), Eq. (5.58), and Eq. (5.59). The performance of the baseline adaptive DSMC and adaptive DSMC with incorporated implementation uncertainties are shown in Figure 5.11 for tracking the desired speed profile under $200 \mathrm{~ms}$ of sampling time and quatization level of 10-bit. As it can be observed, the adaptive DSMC with predicted uncertainties is able to significantly improve the tracking performance by $63 \%$ compared to the baseline adaptive DSMC.

\subsubsection{Motor First Order Adaptive DSMC Real-Time Verification}

The designed controllers in the previous section with adaptation mechanisms are tested in a PIL setup, previously shown in Figure 3.6, to verify the performances of the adaptive DSMCs in real-time. Figure 5.12 and Figure 5.13 show the results of real-time PIL testing of the proposed adaptive DSMCs with incorporated ADC uncertainties on control signals for the DC motor and the engine case studies, respectively. The results verify that the adaptive DSMC is able to (i) remove the uncertainty in 


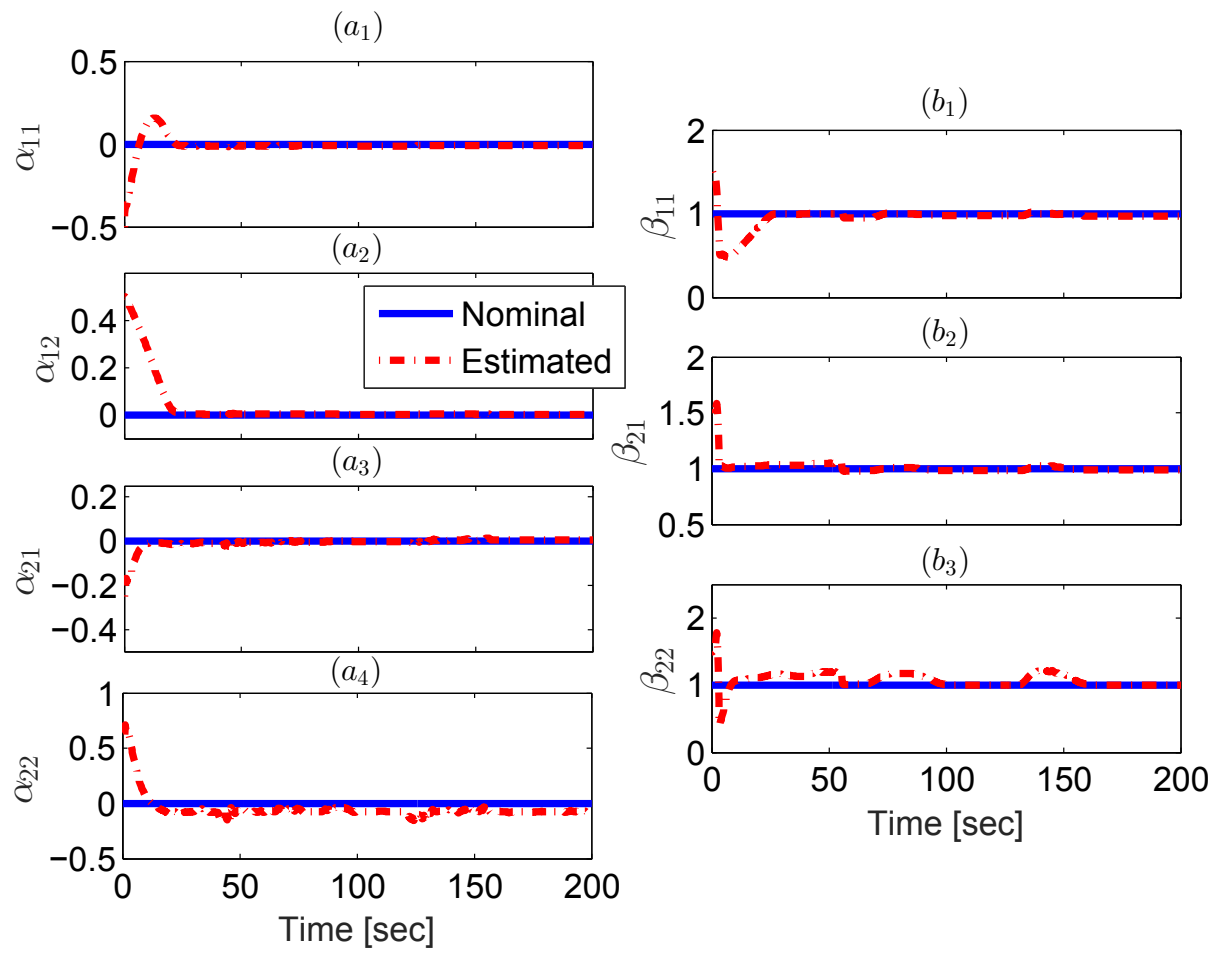

Figure 5.10: Convergence results of the unknown additive $(\alpha)$ and multiplicative $(\beta)$ terms in the DC motor model (200 ms sampling time and 10-bit quantization level).

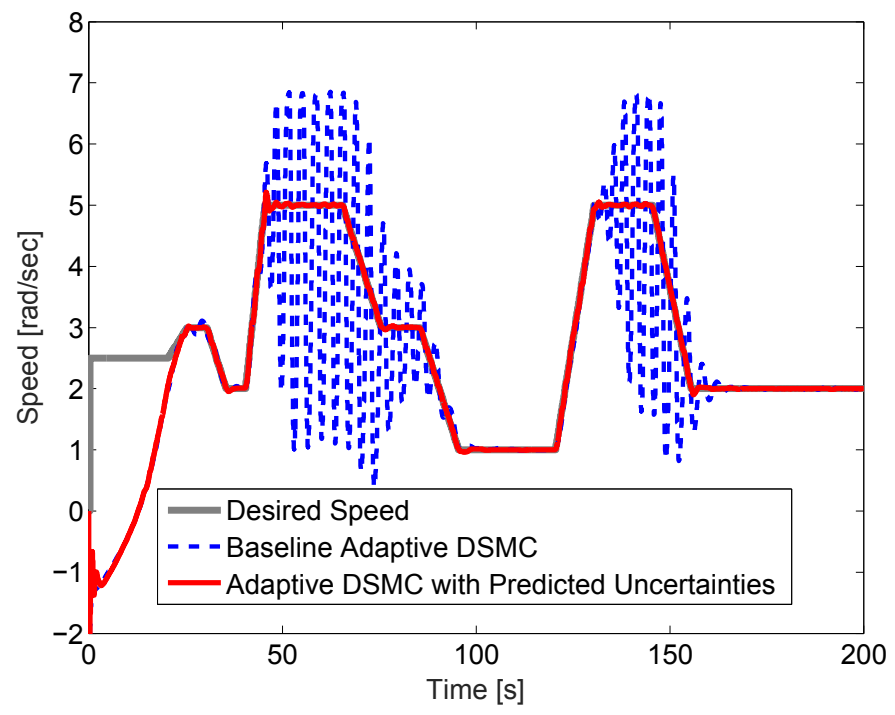

Figure 5.11: DC motor speed control using the baseline adaptive DSMC and the modified adaptive DSMC with predicted ADC uncertainties (sampling time: $200 \mathrm{~ms}$, quantization level: 10-bit). 
the model fast, (ii) track all the desired trajectories under ADC uncertainties, and (iii) operate in real-time since it is computationally efficient.

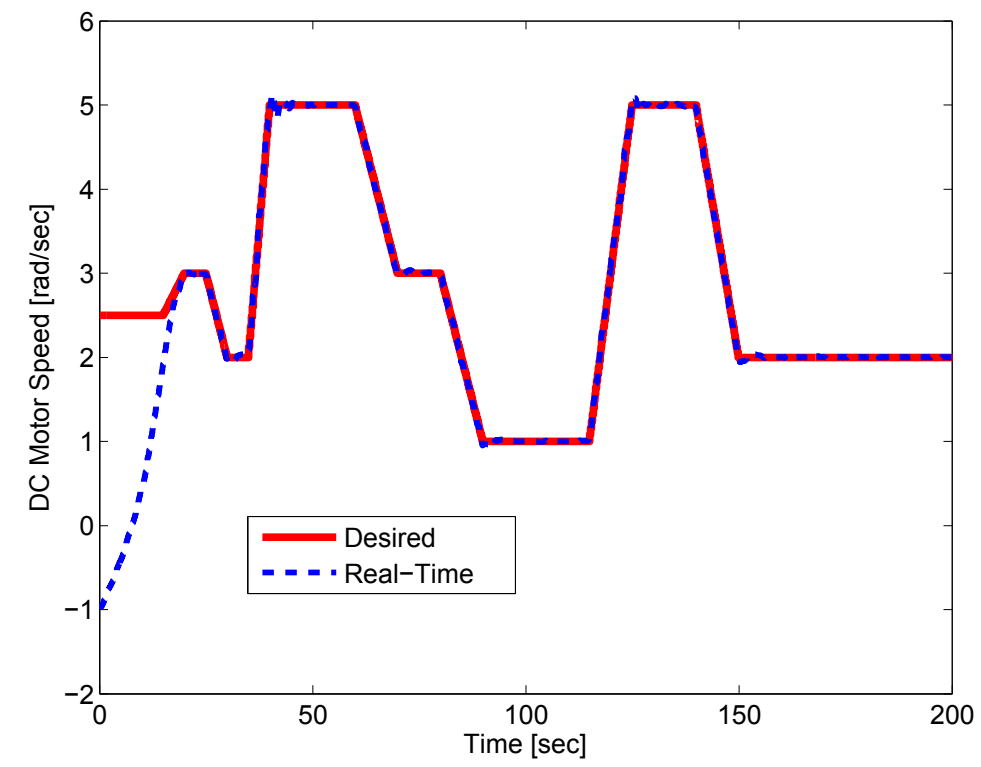

Figure 5.12: Real-time verification result of adaptive robust DSMC for the DC motor speed control (200 ms sampling time and 10-bit quantization level).

\subsection{Summary and Conclusion}

A novel first order adaptive discrete sliding mode controller (DSMC) design for a general class of linear and nonlinear systems was proposed in this chapter. The ADC imprecisions were handled via the online uncertainty prediction and propagation mechanism from Chapter 4. Next, the DSMC design was examined under multiplicative and additive types of model uncertainties. A discrete Lyapunov argument was employed to design adaptation laws to compensate for the unknown uncertainty terms in the controller structure. The final controller is an adaptive robust DSMC 
(a)

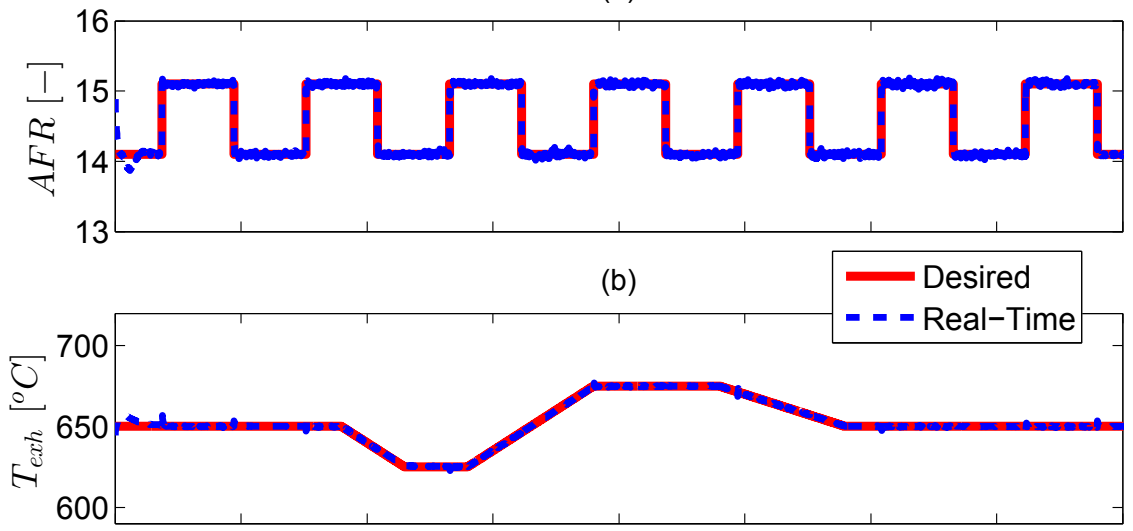

(c)

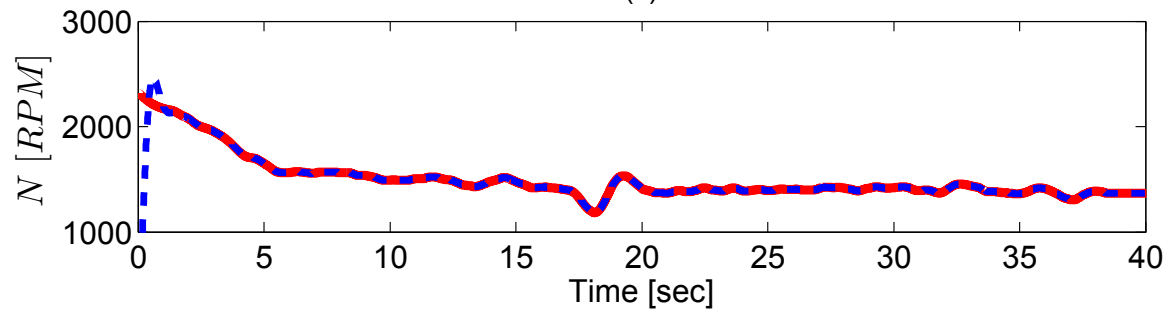

Figure 5.13: Real-time verification results of the adaptive robust DSMC for the engine control case study (10 ms sampling time and 10-bit quantization level).

with incorporated ADC imprecisions which can handle both model and implementation uncertainties. The PIL testing was performed to verify the real-time performance of the proposed DSMCs for two case studies, including DC motor speed regulation and automotive engine control. Real-time experimental and simulation results in this study show:

1. The adaptation laws are computationally efficient for real-time operation. The proposed adaptation laws are able to remove quickly the errors in the modeled dynamics. Although increasing the sampling time raises the required time for the adaptation laws to remove the errors in the model, these errors can be removed permanently in less than 200 time instants for both case studies. For 
the DC motor case study, all the additive and multiplicative unknown parameters were compensated in $30 \mathrm{sec}$ (sampling time $=200 \mathrm{~ms}$ ). On the other side, under $10 \mathrm{~ms}$ of sampling time, the uncertainties in the engine plant model were removed in less than 2 sec.

2. For the linear system case study, DC motor speed regulation, the robustness characteristics of the adaptive DSMC against sampling and quantization imprecisions was improved by $63 \%$ upon incorporating the predicted ADC uncertainties into the controller equations.

3. For the engine control problem, when the sampling time was increased from 10 $m s$ to $35 \mathrm{~ms}$, the tracking errors from the baseline adaptive DSMC increased by 5-10 times. Additionally, by changing the ADC quantization level from 10 bit to 6 bit, the tracking errors of the baseline controller rose by up to 10 times. Similar to the DC motor case study, incorporating the predicted ADC imprecisions into the adaptive DSMC improved the tracking errors by $50-60 \%$ compared to the baseline controller. 


\section{Chapter 6}

\section{Second Order SISO/MIMO Adaptive DSMC1}

\subsection{Introduction}

Converting a higher dimensional tracking control problem into a lower dimensional stabilization control problem is the key feature of sliding mode control (SMC) [57]. SMC shows robust characteristics against external disturbances and model uncertainty/mismatch, while requiring low computational efforts. However, there are challenging issues that arise during implementation of SMC on digital processors, which limit the real-time application of SMC. The two well-recognized challenging issues include: (i) high frequency oscillations due to chattering phenomenon [95, and (ii) implementation imprecisions due to the analog-to-digital (ADC) converter unit (Chapters 2 and 4 .

\footnotetext{
${ }^{1}$ The material of this chapter has been submitted to IEEE Transactions on Control Systems Technology and ASME 2017 Dynamic Systems and Control Conference.
} 
The concept of higher order sliding modes for continuous-time systems is shown to be an effective approach for reducing the oscillation due to chattering. This approach was first introduced in the 1980s [96]. The basic idea of the higher order SMC is to not only steer the sliding function to the sliding manifold, but also drive all the higher order derivatives of the sliding variable to zero. Higher order SMC reduces the high frequency oscillations by transferring the chattering caused by the discontinuity to the higher order sliding mode derivatives. At the end, the control input of a continuous-time chattering-free $\mathcal{N}^{\text {th }}$-order SMC is found by integrating the $\mathcal{N}-1^{\text {th }}$ derivative of the input for $\mathcal{N}-1$ times [96]. Higher order SMC leads to less oscillations; however, it adds complexity to the calculations. Moreover, it has been shown in [97, 98. that converting the continuous-time SMC to a discrete sliding mode controller (DSMC), by using an implicit Euler discretization, allows for a drastic decrease in the chattering in both the input and the output. Thus, according to [99], which presents a second order DSMC, the idea of higher order DSMC can be an ideal solution for the chattering problem by taking advantage of characteristics of the higher order SMC and discretized SMC.

In addition to the high frequency oscillations issue, as it was discussed with details in previous chapters, the SMC structure allows for further modification to improve the controller robustness against ADC (sampling and quantization) imprecisions, and compensate for the uncertainties within the plant model [6]. 
The second order DSMC in [99] is formulated for linear systems without consideration of the uncertainties in the model. Moreover, the study in [99] lacks the stability analysis of the closed-loop system. On the other side, the adaptive DSMC from [5, 6, 62] is limited to the first order sliding mode controller. To this end, in this chapter, a new adaptive second order DSMC formulation is developed for a general class of single-input single-output (SISO) and multiple-input multiple-output (MIMO) uncertain nonlinear systems. Moreover, the asymptotic stability of the new controller is guaranteed via a Lyapunov stability argument and by invoking the new Invariance Principle for nonlinear systems with discontinuity.

The contribution of this chapter is threefold. First, a new second order DSMC is formulated for a general class of nonlinear affine systems. Second, the proposed controller is extended to handle an unknown uncertainty within the plant model using a discrete Lyapunov stability argument that also guarantees the asymptotic stability of the closed-loop system. Third, this chapter presents the first application of the second order DSMC for the combustion engine and DC motor control problems. The proposed second order DSMC not only demonstrates robust behavior against data sampling and quantization imprecisions compared to a first order DSMC, but also removes the uncertainties in the model quickly and steers the dynamics to their nominal values. 


\subsection{SISO Second Order Sliding Mode Control}

\subsubsection{Continuous-Time Second Order SMC}

A general class of continuous-time SISO nonlinear systems can be expressed as follows:

$$
\dot{x}=f(t, x, u)
$$

where $x \in \mathbb{R}^{r}$, and $u \in \mathbb{R}$ are the state, and the input variables, respectively. The sliding mode order is the number of continuous successive derivatives of the differentiable sliding variable $s$, and it is a measure of the degree of smoothness of the sliding variable in the vicinity of the sliding manifold. For the continuous-time systems, the $\mathcal{N}^{\text {th }}$ order sliding mode is determined by the following equalities [100]:

$$
s(t, x)=\dot{s}(t, x)=\ddot{s}(t, x)=\ldots=s^{\mathcal{N}-1}(t, x)=0
$$

The sliding variable $(s)$ is defined as the difference between desired $\left(x_{d}\right)$ and measured signal $(x)$ :

$$
s(t, x)=x(t)-x_{d}(t)
$$

For the second order SMC design, the first derivative is calculated from the state equation (Eq. (6.1)), and the second derivative of the sliding variable is calculated as follows: 


$$
\begin{gathered}
\ddot{s}(t, x, u)=\frac{\partial}{\partial t} \dot{s}(t, x, u)+\frac{\partial}{\partial x} \dot{s}(t, x, u) f(t, x, u) \\
+\frac{\partial}{\partial u} \dot{s}(t, x, u) \dot{u}(t)
\end{gathered}
$$

A new sliding variable $(\xi)$ is defined according to $s$ and $\dot{s}$ :

$$
\xi(t, x)=\dot{s}(t, x)+\beta s(t, x), \beta>0
$$

Eq. 6.5 describes the sliding surface of a system with a relative order equal to one, in which the input is $\dot{u}$ and output is $\xi(t, x)$ [101]. The equivalent control input is obtained by solving the following equation:

$$
\dot{\xi}(t, x)=0 \Rightarrow \ddot{s}(t, x)+\beta \dot{s}(t, x)=0
$$

which according to the sliding variable definition needs the second derivative of the state variable:

$$
\begin{gathered}
\ddot{x}(t)=\frac{\partial}{\partial t} f(t, x, u)+\frac{\partial}{\partial x} f(t, x, u) f(t, x, u) \\
+\frac{\partial}{\partial u} f(t, x, u) \dot{u}(t)
\end{gathered}
$$

Substituting Eq. (6.3) and (6.7) in Eq. (6.6) leads to:

$$
\begin{gathered}
\left(\frac{\partial}{\partial t} f(t, x, u)+\left(\frac{\partial}{\partial x} f(t, x, u)\right) f(t, x, u)+\right. \\
\left.\frac{\partial}{\partial u} f(t, x, u) \dot{u}(t)-\ddot{x}_{d}(t)\right)+\beta \dot{s}(x, t)=0
\end{gathered}
$$

Next, $\dot{u}$ is obtained from Eq. (6.8): 


$$
\begin{gathered}
\dot{u}(t)=\frac{1}{\frac{\partial}{\partial u} f(t, x, u)}\left(-\frac{\partial}{\partial t} f(t, x, u)\right. \\
\left.-\left(\frac{\partial}{\partial x} f(t, x, u)\right) f(t, x, u)+\ddot{x}_{d}(t)-\beta \dot{s}(x, t)\right)
\end{gathered}
$$

and finally the equivalent control input $\left(u_{e q}\right)$ which satisfies the second order sliding mode condition is:

$$
u_{e q}(t)=\int \dot{u}(t) d t
$$

The controller robustness against external (i.e., ADC) uncertainties is ensured by inclusion of a switching function $\left(u_{s w}\right)$ [57]:

$$
u_{s w}(t)=-\Omega \times \operatorname{sgn}(\xi(t, x)), \quad \Omega>0
$$

Thus, the final control input of the second order SMC is obtained based on Eq. (6.10) and 6.11 as follows:

$$
u(t)=u_{e q}+u_{s w}(t)=u_{e q}-\Omega \times \operatorname{sgn}(\xi(t, x))
$$

where $\Omega>0$ could be a constant or variable gain. This approach guarantees asymptotic convergence of the sliding variable and its derivative to zero in finite time [99].

\subsubsection{Discrete-Time Adaptive Second Order SMC}

The affine SISO form of the nonlinear system in Eq. (6.1) with an unknown multiplicative term $(\alpha)$ can be presented using the following state space equation: 


$$
\dot{x}(t)=\alpha f(x(t))+g(x(t)) u(t)
$$

where $g(x(t))$ is a non-zero input coefficient and $f(x(t))$ represents the dynamics of the plant and does not depend on the inputs. $\alpha$, which represents the errors/mismatches in the plant model, is unknown and constant. The continuous-time model in Eq. 6.13 is discretized by utilizing the first order Euler approximation:

$$
x(i+1)=T \alpha f(x(i))+T g(x(i)) u(i)+x(i)
$$

in which $T$ is the sampling time. Similar to Eq. (6.5), a new discrete sliding variable is defined:

$$
\xi(i)=s(i+1)+\beta s(i), \beta>0
$$

where $s(i)=x(i)-x_{d}(i)$ and $\beta$ is a constant second order sliding mode gain.

The equivalent control input of the second order DSMC should satisfy the second order discrete sliding mode criteria, which is [99]:

$$
\xi(i+1)=\xi(i)=0
$$

Applying the second order sliding mode condition from Eq. (6.16) to the nonlinear system in Eq. 6.14 results in the following equivalent control input $\left(u_{e q}\right)$ :

$$
u_{e q}(i)=\frac{1}{g T}\left(-T \hat{\alpha}(i) f(x(i))-x(i)+x_{d}(i+1)-\beta s(i)\right)
$$

where $\hat{\alpha}(i)$ is the estimation of the unknown multiplicative uncertainty term in the 
plant's model. By incorporating the control law $\left(u_{e q}\right)$ into the second order sliding variable $(\xi)$, we have:

$$
\xi(i)=T f(x(i))(\alpha-\hat{\alpha}(i))=T f(x(i)) \tilde{\alpha}(i)
$$

$\tilde{\alpha}(i)$ is the error in estimating the unknown multiplicative term $(\tilde{\alpha}(i)=\alpha-\hat{\alpha}(i))$. Next, a Lyapunov stability analysis is conducted to (i) determine the stability of the closed-loop system, and (ii) derive the adaptation law to remove the uncertainty in the model. To this end, the following Lyapunov candidate function is proposed:

$$
V(i)=\frac{1}{2}\left(s^{2}(i+1)+\beta s^{2}(i)\right)+\frac{1}{2} \rho_{\alpha}\left(\tilde{\alpha}^{2}(i+1)+\beta \tilde{\alpha}^{2}(i)\right)
$$

where $\rho_{\alpha}>0$ is a tunable adaptation gain chosen for the numerical sensitivity of the unknown parameter estimation. As can be seen from Eq. 6.19, the proposed Lyapunov function is positive definite and quadratic with respect to the sliding variable $(s(i))$ and the unknown parameter estimation error $(\tilde{\alpha}(i))$. Asymptotic and finitetime convergence of $s$ and $\tilde{\alpha}$ to zero are the desired conditions. To guarantee the latter conditions, in the discrete time domain, the difference function of $V$ should be at least negative semi-definite to ensure the stability of the closed-loop system [5]. The Lyapunov difference function is calculated using a Taylor series expansion: 


$$
\begin{gathered}
V(i+1)=V(i)+\frac{\partial V(i)}{\partial s(i)} \Delta s(i) \\
+\frac{\partial V(i)}{\partial s(i+1)} \Delta s(i+1)+\frac{\partial V(i)}{\partial \tilde{\alpha}(i)} \Delta \tilde{\alpha}(i) \\
+\frac{\partial V(i)}{\partial \tilde{\alpha}(i+1)} \Delta \tilde{\alpha}(i+1)+\frac{1}{2} \frac{\partial^{2} V(i)}{\partial s^{2}(i)} \Delta s^{2}(i) \\
+\frac{1}{2} \frac{\partial^{2} V(i)}{\partial s^{2}(i+1)} \Delta s^{2}(i+1)+\frac{1}{2} \frac{\partial^{2} V(i)}{\partial \tilde{\alpha}^{2}(i)} \Delta \tilde{\alpha}^{2}(i)+\frac{1}{2} \frac{\partial^{2} V(i)}{\partial \tilde{\alpha}^{2}(i+1)} \Delta \tilde{\alpha}^{2}(i+1)+\ldots
\end{gathered}
$$

where:

$$
\begin{gathered}
\Delta s(i) \equiv s(i+1)-s(i) \\
\Delta \tilde{\alpha}(i) \equiv \tilde{\alpha}(i+1)-\tilde{\alpha}(i)
\end{gathered}
$$

The first order Lyapunov difference function $(\Delta V(i)=V(i+1)-V(i))$ can be obtained by substituting the values of the partial derivatives into Eq. (6.20):

$$
\begin{gathered}
\Delta V(i)=\beta s(i) \Delta s(i)+s(i+1) \Delta s(i+1) \\
+\rho_{\alpha} \beta \tilde{\alpha}(i) \Delta \tilde{\alpha}(i)+\rho_{\alpha} \tilde{\alpha}(i+1) \Delta \tilde{\alpha}(i+1) \\
+\frac{1}{2} \beta \Delta s^{2}(i)+\frac{1}{2} \Delta s^{2}(i+1)+\frac{1}{2} \rho_{\alpha} \beta \Delta \tilde{\alpha}^{2}(i)+\frac{1}{2} \rho_{\alpha} \Delta \tilde{\alpha}^{2}(i+1)+\ldots
\end{gathered}
$$

Eq. 6.22 can be simplified after substituting Eq. 6.18) and 6.21) at $i$ and $i+1$ time steps:

$$
\begin{gathered}
\Delta V(i)=-\beta(\beta+1) s^{2}(i)-(\beta+1) s^{2}(i+1) \\
+\beta s(i) T f \tilde{\alpha}(i)+\rho_{\alpha} \beta \tilde{\alpha}(i) \Delta \tilde{\alpha}(i) \\
+s(i+1) T f \tilde{\alpha}(i+1)+\rho_{\alpha} \tilde{\alpha}(i+1) \Delta \tilde{\alpha}(i+1) \\
+O\left(\Delta s^{2}(i), \Delta s^{2}(i+1), \Delta \tilde{\alpha}^{2}(i), \Delta \tilde{\alpha}^{2}(i+1)\right)+\ldots
\end{gathered}
$$


which yields:

$$
\begin{gathered}
\Delta V(i)=-(\beta+1)\left(s^{2}(i+1)+\beta s^{2}(i)\right) \\
+\rho_{\alpha} \beta \tilde{\alpha}(i)\left(\frac{s(i) T f}{\rho_{\alpha}}+\Delta \tilde{\alpha}(i)\right) \\
+\rho_{\alpha} \tilde{\alpha}(i+1)\left(\frac{s(i+1) T f}{\rho_{\alpha}}+\Delta \tilde{\alpha}(i+1)\right) \\
+O\left(\Delta s^{2}(i), \Delta s^{2}(i+1), \Delta \tilde{\alpha}^{2}(i), \Delta \tilde{\alpha}^{2}(i+1)\right)
\end{gathered}
$$

in which all the higher order $(>2)$ terms are zero. In Eq. 6.24), the first term is a negative definite when $\beta>0$. As explained earlier, the Lyapunov difference function should be at least negative semi-definite. To this end, the second and third terms in Eq. (6.24) should become zero, which leads to the following adaptation law:

$$
\tilde{\alpha}(i+1)=\tilde{\alpha}(i)-\frac{T s(i) f(x(i))}{\rho_{\alpha}}
$$

It should be noticed that the adaptation law for handling the modeling uncertainties for the second order DSMC (Eq. (6.25) has the same structure of the proposed adaptation laws for the first order DSMC (Eq. (5.14). By applying Eq. (6.25), as the adaptation law, the Lyapunov difference function becomes:

$$
\begin{gathered}
\Delta V(i)=-(\beta+1)\left(s^{2}(i+1)+\beta s^{2}(i)\right) \\
+O\left(\Delta s^{2}(i), \Delta s^{2}(i+1), \Delta \tilde{\alpha}^{2}(i), \Delta \tilde{\alpha}^{2}(i+1)\right)
\end{gathered}
$$

The proposed equivalent control input (Eq. 6.17) ) of the second order DSMC is next evaluated on Gao's reaching law [102]. To this end, we begin with the first 
order continuous-time SMC, which can be realized by applying the following sliding reaching law [57, 103]:

$$
\dot{s}(t)=-\lambda s(t)-\varepsilon \times \operatorname{sgn}(s(t)), \quad \lambda>0, \varepsilon>0
$$

Eq. (6.27) can be discretized using the first order Euler approximation as follows:

$$
\begin{gathered}
\frac{s(i+1)-s(i)}{T}=-\lambda s(i)-\varepsilon \times \operatorname{sgn}(s(i)) \rightarrow \\
s(i+1)=(1-T \lambda) s(i)-T \varepsilon \times \operatorname{sgn}(s(i))
\end{gathered}
$$

where the $1>1-T \lambda>0$ condition should be met to guarantee that states of the system will move monotonically toward the switching plane $(s=0)$, and cross it in finite time [102]. Next, the first order sliding variable $(s)$ in Eq. 6.28) is replaced with the second order sliding variable $(\xi)$ :

$$
\xi(i+1)=(1-T \lambda) \xi(i)-T \varepsilon \times \operatorname{sgn}(\xi(i)), \quad(1-T \lambda)>0
$$

By recalling Eq. 6.15 we have:

$$
\begin{gathered}
\xi(i+1)=s(i+2)+\beta s(i+1), \quad \beta>0 \\
\rightarrow s(i+2)=\xi(i+1)-\beta s(i+1)
\end{gathered}
$$

Then, substituting Eq. 6.29 in to Eq. 6.30 yields:

$$
s(i+2)=(1-T \lambda)(s(i+1)+\beta s(i))-\beta s(i+1)-T \varepsilon \times \operatorname{sgn}(\xi(i))
$$

and, finally Eq. 6.31) can be simplified as follows [103]: 


$$
\begin{gathered}
s(i+2)=\phi_{1}(T \alpha f(x(i))+\operatorname{Tg}(x(i)) u(i)+x(i) \\
\left.-x_{d}(i+1)\right)+\phi_{2} s(i)-T \varepsilon \times \operatorname{sgn}(\xi(i))
\end{gathered}
$$

where $\phi_{1}=1-T \lambda-\beta$ and $\phi_{2}=\beta(1-T \lambda)$. Next, the calculated equivalent control input of the second order DSMC from Eq. 6.17) along with the so-called switching control input $\left(u_{s w}\right)$ are substituted in Eq. 6.32:

$$
\begin{gathered}
s(i+2)=\phi_{1}(x(i)+T \alpha f(x(i))+ \\
g T\left(\frac{1}{g T}\left(-T \hat{\alpha} f(x(i))-x(i)+x_{d}(i+1)-\beta s(i)\right)+u_{s w}\right) \\
\left.-x_{d}(i+1)\right)+\phi_{2} s(i)-T \varepsilon \times \operatorname{sgn}(\xi(i))
\end{gathered}
$$

which can be simplified as follows:

$$
s(i+2)=\phi_{1}\left(T \tilde{\alpha} f(x(i))-\beta s(i)+T g(x(i)) u_{s w}\right)+\phi_{2} s(i)-T \varepsilon \times \operatorname{sgn}(\xi(i))
$$

In [102], it has been stated that once the state trajectory has crossed the sliding surface, the state trajectory will cross the surface again in every successive sampling period. This means:

$$
\operatorname{sgn}(\xi(i))=-\operatorname{sgn}(\xi(i-1))
$$

thus, Eq. 6.34 can be written as:

$$
\begin{aligned}
s(i+2)= & \phi_{1}\left(T \tilde{\alpha} f(x(i))-\beta s(i)+T g(x(i)) u_{s w}\right) \\
& +\phi_{2} s(i)+T \varepsilon \times \operatorname{sgn}(\xi(i-1))
\end{aligned}
$$


Finally, by incorporating the values of $\phi_{1}$ and $\phi_{2}$ we have:

$$
s(i+2)=\beta^{2} s(i)+\phi_{1}\left(T \tilde{\alpha} f(x(i))+T g(x(i)) u_{s w}\right)+T \varepsilon \times \operatorname{sgn}(\xi(i-1))
$$

If the switching control input is selected to be

$$
u_{s w}=-\frac{\varepsilon}{\phi_{1} g(x(i))} \times \operatorname{sgn}(\xi(i-1))
$$

in which $g(x(i)) \neq 0$ and $\phi_{1} \neq 0$, then, Eq. 6.37) becomes:

$$
s(i+2)=\beta^{2} s(i)+\phi_{1}(T \tilde{\alpha} f(x(i)))
$$

Let assume that by using Eq. (6.25), the uncertainty in the model will be removed. This means that the error in estimating the unknown parameter converges to zero (i.e., $\tilde{\alpha}(i+1)=\tilde{\alpha}(i)=0)$. Thus:

$$
s(i+2) \approx \beta^{2} s(i)
$$

Eq. 6.40 shows that, if $1>\beta>\beta^{2}>0$, the proposed equivalent control input of the second order DMSC along with the switching function in Eq. (6.38) fulfills Gao's discrete sliding mode reaching law, which guarantees finite time convergence of the system's states to the sliding manifold [102].

In the next step, by expanding the second order terms $(O()$.$) in Eq. 6.26), and$ assuming a small enough sampling time $(T)$, which means all terms that contain $T^{2}$ can be neglected, Eq. 6.26 can be re-arranged as follows: 


$$
\begin{gathered}
\Delta V(i)=-\frac{1}{2}\left(\beta s^{2}(i+1)+\beta s^{2}(i)+2 \beta^{2} s^{2}(i)\right. \\
\left.+s^{2}(i+1)-s^{2}(i+2)\right)-\beta s(i+1) s(i)-s(i+2) s(i+1)
\end{gathered}
$$

in which, it is assumed that the uncertainty in the model is compensated by applying Eq. 6.25). According to the second order sliding variable $\xi$ definition (Eq. 6.16) ) and Eq. 6.40), $s(i+1)$ and $s(i+2)$ can be replaced by $-\beta s(i)$ and $\beta^{2} s(i)$, respectively. By doing these replacements, Eq. 6.41 can be simplified as:

$$
\Delta V(i)=-\frac{1}{2} \beta\left(-\beta^{3}-\beta^{2}+\beta+1\right) s^{2}(i)
$$

$-\beta^{3}-\beta^{2}+\beta+1$ is positive if $1>\beta>0$. In other words, if $1>\beta>0$, then $\Delta V(i) \leq 0$, which guarantees the stability of the system $(\xi(i) \rightarrow 0)$. Of interesting note is the $1>\beta>0$ constraint which is consistent with the earlier concluded condition on $\beta$ to make sure Gao's sliding reaching law is fulfilled by the equivalent control input of the second order DSMC.

We showed that by using $u_{e q}$ from Eq. 6.17), $u_{s w}$ from Eq. 6.38), and the adaptation law from Eq. 6.25), the negative semi-definite condition of the Lyapunov difference function is guaranteed. This means that the sliding variable (the tracking error, $s$ ) converges to zero, and the error in estimating the unknown parameter $(\tilde{\alpha})$ is at least bounded. Moreover, according to Eq. (6.40), since $1>\beta>0$, it is obvious that $1>\beta>\beta^{2}>0$. Because the Lyapunov difference function is at least negative-semi definite, it can be concluded that: 


$$
s(i)>s(i+1)>s(i+2)
$$

and, $s(i+2) \neq s(i+1) \neq s(i)$ unless $s(i+2)=s(i+1)=s(i)=0$. Therefore, Eq. 6.43 shows that the second order DSMC guarantees asymptotic decrease of $s$ for the next two steps $(i, i+1, i+2)$. However, in order to guarantee the global asymptotic convergence and stability characteristics of the second order DMSC, it is required to show that all the higher order Lyapunov difference functions vanish as $s \rightarrow 0$ [6, 59]. To this end, the analysis begins with the second order Lyapunov difference function $(\Delta V(i+1)-\Delta V(i))$. Let us first define a new term, $\varrho=\beta\left(-\beta^{3}-\beta^{2}+\beta+1\right)$, where $\varrho>0$. With the definition of $\varrho, \Delta V(i+1)-\Delta V(i)$ is calculated as follows:

$$
\Delta V(i+1)-\Delta V(i) \approx \frac{1}{2} \varrho s^{2}(i+1)-\frac{1}{2} \varrho s^{2}(i) \approx \frac{1}{2} \varrho\left(s^{2}(i+1)-s^{2}(i)\right)
$$

The objective is to show that $\Delta V(i+1)-\Delta V(i)=0$ when $s(i)=0$. According to Eq. 6.44, it is obvious that $\Delta V(i+1)-\Delta V(i)=0$ if $s^{2}(i+1)-s^{2}(i)=0$. $s^{2}(i+1)-s^{2}(i)=0$ if either of the following conditions is met:

$$
\begin{aligned}
& s(i+1)=s(i)=0 \quad(I) \\
& s(i+1)=s(i) \neq 0 \quad(I I)
\end{aligned}
$$

Eq. 6.42 says that if $s(i)=0$, then $\Delta V(i)=0$. If one assumes that the unknown uncertainty term is removed from the model using the proposed adaptation algorithm, the second order sliding mode condition denotes that $\xi(i+1)=\xi(i)=0$, which means $s(i+1)=-\beta s(i)$. Thus, if $s(i)=0, s(i+1)$ also becomes zero, and consequently 
$\Delta V(i+1)-\Delta V(i)=0$. This means that condition (I) in Eq. 6.45 is realizable.

According to Eq. 6.43, $s(i+1)<s(i)$. This means $s(i+1) \neq s(i)$ unless $s(i+1)=$ $s(i)=0$. Thus, the condition $(I I)$ in Eq. 6.45 cannot be true and only condition $(I)$ is feasible. According to condition $(I)$, if $s(i)=0$, not only $\Delta V(i)=0$, but also $\Delta V(i+1)-\Delta V(i)=0$. Next, the third Lyapunov difference function should be calculated:

$$
\begin{gathered}
{[\Delta V(i+2)-\Delta V(i+1)]-[\Delta V(i+1)-\Delta V(i)]} \\
\approx \frac{1}{2} \Gamma\left(s^{2}(i+2)-2 s^{2}(i+1)+s^{2}(i)\right)
\end{gathered}
$$

Again, since $s(i+2)<s(i+1)<s(i), s(i+2) \neq s(i+1) \neq s(i)$ unless $s(i+2)=s(i+$ $1)=s(i)=0$. Thus, if $s(i)=0$, not only $s(i+1)=s(i+2)=0$, but also $\Delta V(i)=0$, $\Delta V(i+1)-\Delta V(i)=0$, and $(\Delta V(i+2)-\Delta V(i+1))-(\Delta V(i+1)-\Delta V(i))=0$. In a same manner as the first, second, and third order difference functions, it can be shown that higher order Lyapunov difference functions $(>3)$ become zero if, and only if $s(i)=0$ [6, 59]. This is a key conclusion that allows for proof of the global asymptotic stability by invoking the new Invariance Principle for nonautonomous systems [104].

Continuity is one of the required conditions for the LaSalle's Invariance Principle [105] to conclude the asymptotic stability with respect to a negative semi-definite derivative of a positive definite Lyapunov function. LaSalle's Invariance Principle has been extended in [104] to nonlinear systems with discontinuity. The extension of LaSalle's 
Invariance Principle to discrete systems, which is called the new Invariance Principle theorem, removes the continuity requirement, and allows us to conclude the asymptotic stability with respect to a negative semi definite difference function of a positive definite Lyapunov discrete equation. It was shown that when $s(i)=0$, the Lyapunov difference function, all the future values of the sliding variable, and higher order Lyapunov differences become zero. Therefore, all the trajectories of the system approach the set defined by $\Delta V(i) \equiv 0$. Since $s(i)=0$ and $\tilde{\alpha}(i)=0$ are the only trajectories which satisfy the nonlinear uncertain system equations, this trajectory $(s(i)=0, \tilde{\alpha}(i)=0)$ is a limit point, and also an equilibrium point, of the closed-loop system [6, 59].

Since the Lyapunov difference function cannot be negative for any unlimited period of time, according to the new Invariance Principle theorem [104], $\Delta V$ must be identically zero at any limit point [94]. Let define two new domains:

$$
\begin{gathered}
\Omega_{0}=\left\{x \mid V(x) \leq V\left(x_{0}\right)\right\} \\
\left.\Omega_{i}=\{x \mid \Delta V(x) \equiv 0)\right\}
\end{gathered}
$$

where $x_{0}=x(0)$ is the initial condition. The negative semi definite condition of the Lyapunov difference function means that all system states are bounded and contained within the domain $\Omega_{0}$ [104]. For a small enough sampling time, if the following condition holds [104]:

- $|x(i)+T f(x(i))+T g(x(i)) u(i)|$ is bounded for any bounded $x$ 
then, $s(i)=\tilde{\alpha}(i)=0$, which is the only limit point (equilibrium point) of the system, belongs to $\Omega_{f}=\Omega_{0} \bigcap \Omega_{i}$. Therefore, $s(i)$ and $\tilde{\alpha}(i) \rightarrow 0$ as $i \rightarrow \infty$. Thus, according to the new Invariance Principle theorem [104], the asymptotic convergence of the two variables to zero with a positive definite Lyapunov function and a negative semidefinite Lyapunov difference equation is concluded.

Overall, the control input of the second order DSMC is:

$$
u(i)=u_{e q}(i)+u_{s w}(i)
$$

where $u_{e q}$ and $u_{s w}$ are calculated according to Eq. (6.17), and Eq. (6.38), respectively. The gain of the switching function $(\operatorname{sgn}(\xi(i-1)))$, which depends on the DSMC tuning parameters $(\lambda, \beta)$ and $g$ (Eq. (6.13) $)$, is the key to ensure the controller robustness against external uncertainties [6]. By looking into Eq. 6.38, it can be seen that the gain of the switching function has the same unit of the control input. This gain represents the boundary of the external uncertainties on the control signals, i.e. ADC imprecisions [1, 24]. Tuning the switching function gain based on constant $\lambda$ and $\beta$ is hard to achieve. Instead, an online sampling and quantization uncertainty prediction mechanism was proposed in [4] which allows for estimating and propagating the ADC uncertainty bounds on the control signal, and avoids conservative controller design. The switching function with the predicted ADC uncertainty bounds has the following structure:

$$
u_{s w}(i)=-\left|\mu_{u}\right| \operatorname{sgn}(\xi(i-1))
$$


where $\mu_{u}$ is the propagated sampling and quantization imprecisions. $\mu_{u}$ is estimated according to the mechanism presented with details in [4, 6]. In order to avoid possible high frequency chattering which occurs in discrete systems during implementation of the signum function, the signum function is replaced with saturation $(s a t)$ function which provides smoother behaviour:

$$
u_{s w}(i)=-\left|\mu_{u}\right| \operatorname{sat}(s(i)+\beta s(i-1))
$$

\subsection{MIMO Adaptive Second Order DSMC}

The discretized nonlinear system can be expressed in MIMO structure as follows:

$$
\mathbf{x}(i+1)=T \mathbf{f}(\mathbf{x}(i))+T \mathbf{g}(\mathbf{x}(i)) \mathbf{u}(i)+\mathbf{x}(i),
$$

where $x_{k} \subset \mathbf{x} \in \mathbb{R}^{r},(k=1: r)$, and $u_{j} \subset \mathbf{u} \in \mathbb{R}^{h},(j=1: h)$ are the state and control input vectors, respectively. The remaining dynamics are represented as $\mathbf{f}(\mathbf{x}(i))$ and $\mathbf{g}(\mathbf{x}(i))$. A generic first order DSMC for the MIMO system in Eq. 6.51) has been proposed in our previous work [62]. Here, we use the results from [62] to derive the first order sliding surface vector $\left(\mathbf{s}(i)=\left[s_{1}(i), \ldots, s_{h}(i)\right]^{\top}\right)$ based on a system output vector which is defined to be $y_{j} \subset \mathbf{y} \in \mathbb{R}^{h},(j=1: h)$ :

$$
y_{j}(i)=m_{j}(\mathbf{x}(i))
$$

For a system with an output $y_{j}$ that has a relative degree of $\kappa_{j}$, the scalar sliding 
surface $s_{j}(t)$ is defined as:

$$
s_{j}(t)=\left(\frac{d}{d t}+\lambda_{j}\right)^{\kappa_{j}-1}\left(y_{j}(t)-y_{j_{d}}(t)\right)
$$

where $y_{j_{d}} \subset \mathbf{y}_{d} \in \mathbb{R}^{h},(j=1: h)$ is the desired output values. It was shown in [62] that for a MIMO system with a relative degree of $\kappa_{j}\left(\sum_{1}^{h} \kappa_{j}<r\right)$, the discrete dynamics of the first order sliding surface (s) in the presence of the model uncertainties is:

$$
\mathbf{s}(i+1)=\mathbf{s}(i)+T \hat{\mathbf{\Lambda}}+T \mathbf{F a}+T \mathbf{r} \mathbf{u}(i),
$$

where $\mathbf{a} \in \mathbb{R}^{p}$ is a vector of unknown constants in the model and $\mathbf{F} \in \mathbb{R}^{h \times p}$ is known as a data matrix [62]. $\hat{\Lambda}$ is defined as follows:

$$
\hat{\Lambda}=\Lambda-\mathbf{F a}
$$

in which $\boldsymbol{\Lambda}=\left[l_{1}, l_{2}, \ldots, l_{h}\right]^{\top}$, and:

$$
l_{j}=L_{f}^{\kappa_{j}}\left(h_{j}\right)+\sum_{q=\kappa_{j}-1}^{1} c_{j\left(\kappa_{j}-q+1\right) \cdot[}\left[L_{f}^{q}\left(h_{j}\right)-\frac{d^{\kappa_{j}}}{d t^{\kappa_{j}}} y_{j_{d}}^{\left(\kappa_{j}\right)}(t)\right]
$$

and, $L_{f}^{q}\left(h_{j}\right)$ is defined as [105]:

$$
L_{f}^{q}\left(h_{j}\right)=\frac{d^{q} y_{j}(t)}{d t^{q}}
$$

with $c_{j\left(\kappa_{j}-q+1\right)}$ chosen such that all poles are at $-\lambda_{j}$ [59]. In the absence of the model uncertainties, it is obvious that $\hat{\boldsymbol{\Lambda}}=\boldsymbol{\Lambda}$. Here, we assume a relative degree of one $\left(\kappa_{j}=1\right)$ for the output. Thus, according to the sliding surface definition and the relative degree of the outputs, the non-singular $\Upsilon \in \mathbb{R}^{h \times h}$ matrix in Eq. 6.54 becomes: 


$$
\Upsilon=\left[\begin{array}{ccc}
g_{11} & \ldots & g_{1 h} \\
\vdots & \ddots & \\
g_{h 1} & \ldots & g_{h h}
\end{array}\right]
$$

It is assumed that here we deal with a MIMO system that has an output with a relative degree of one. Therefore, according to Eq. (6.53), $s_{j}(i)=y_{j}(i)-y_{d_{j}}(i)$. For the sake of simplicity it can be assumed that $r=h$ (which means $k=j=p=1: r$ ), $m_{i}(\mathbf{x})=x_{i}(i)$, and $y_{d_{k}}(i)=x_{d k}(i)$. The latter assumption means that here, state space variables of the state vector are treated as the output variables, and for each state variable $\left(x_{i}\right)$, a sliding surface $\left(s_{i}\right)$ is defined and it is assumed that a unique control input $\left(u_{i}\right)$, either physical or synthetic, exists for every single sliding surface within the sliding surface vector $(\mathbf{s})$.

Now, the second order sliding surface vector $\left(\boldsymbol{\xi}=\left[\xi_{1}, \ldots, \xi_{r}\right]^{\top}\right)$ is constructed:

$$
\boldsymbol{\xi}(i)=\mathbf{s}(i+1)+\boldsymbol{\beta} \mathbf{s}(i),
$$

where $\mathbf{s}(i+1)$ is calculated with respect to Eq. (6.54) and $\boldsymbol{\beta} \in \mathbb{R}^{r \times r}$ is the positive definite matrix of the second order sliding mode gains. The equivalent control input vector which satisfies the second order sliding mode condition $(\boldsymbol{\xi}(i+1)=\boldsymbol{\xi}(i)=0)$ can be found by solving the following equation for $\mathbf{u}_{e q}$ :

$$
T \Upsilon \mathbf{u}_{e q}(i)=-(\mathbf{I}+\boldsymbol{\beta}) \mathbf{s}(i)-T \hat{\boldsymbol{\Lambda}}-T \mathbf{F} \hat{\mathbf{a}}(i),
$$


where $\hat{\mathbf{a}}(i)$ is the estimation of the unknown constants $\mathbf{a}$. In a similar manner to the SISO controller, upon substitution of Eq. 6.60 into Eq. (6.59), we have:

$$
\boldsymbol{\xi}(i)=T \mathbf{F} \tilde{\mathbf{a}}(i),
$$

where $\tilde{\mathbf{a}}(i)=\mathbf{a}-\hat{\mathbf{a}}(i)$.

Similar to the SISO controller, a discrete Lyapunov analysis is carried out to first converge ã to zero and remove the uncertainty in the model, and second, guarantee the asymptotic stability of the closed loop system. To this end, the argument begins with the following Lyapunov candidate function [62]:

$$
V(i)=\frac{1}{2} \mathbf{S}(i)^{\top} \mathbf{S}(i)+\frac{1}{2} \tilde{\mathbf{a}}(i)^{\top} \boldsymbol{\Gamma} \tilde{\mathbf{a}}(i)
$$

where $\boldsymbol{\Gamma} \in \mathbb{R}^{r \times r}$ is the tunable adaptation symmetric positive matrix. The Lyapunov difference function, $\Delta V(i)=V(i+1)-V(i)$, is obtained by performing the Taylor series expansion on Eq. 6.62). To this end, first we define $\Delta \mathbf{s}$ and $\Delta \tilde{\mathbf{a}}$ vectors as follows:

$$
\begin{gathered}
\Delta \mathbf{s}(i)=\mathbf{s}(i+1)-\mathbf{s}(i) \\
\Delta \tilde{\mathbf{a}}(i)=\tilde{\mathbf{a}}(i+1)-\tilde{\mathbf{a}}(i) .
\end{gathered}
$$

Next, $\Delta V(i)$ is calculated: 


$$
\begin{aligned}
& \Delta V(i)=\frac{\partial V(i)}{\partial \mathbf{s}(i)} \Delta \mathbf{s}(i)+\frac{\partial V(i)}{\partial \tilde{\mathbf{a}}(i)} \Delta \tilde{\mathbf{a}}(i) \\
& +\frac{1}{2} \frac{\partial^{2} V(i)}{\partial\left(\mathbf{s}(i)^{\top} \mathbf{s}(i)\right)}\left(\Delta \mathbf{s}(i)^{\top} \Delta \mathbf{s}(i)\right. \\
& +\frac{1}{2} \frac{\partial^{2} V(i)}{\partial\left(\tilde{\mathbf{a}}(i)^{\top} \tilde{\mathbf{a}}(i)\right)}\left(\Delta \tilde{\mathbf{a}}(i)^{\top} \Delta \tilde{\mathbf{a}}(i)\right)+\ldots
\end{aligned}
$$

where all the higher order $(>2)$ partial derivatives and also second order cross derivative are zero. The first order partial derivatives in Eq. (6.64) are:

$$
\frac{\partial V(i)}{\partial \mathbf{s}(i)}=\mathbf{s}(i)^{\top}, \frac{\partial V(i)}{\partial \tilde{\mathbf{a}}(i)}=\tilde{\mathbf{a}}(i)^{\top} \boldsymbol{\Gamma},
$$

and the second order partial derivatives are:

$$
\frac{\partial^{2} V(i)}{\partial\left(\mathbf{s}(i)^{\top} \mathbf{s}(i)\right)}=\mathbf{I}, \frac{\partial^{2} V(i)}{\partial\left(\tilde{\mathbf{a}}(i)^{\top} \tilde{\mathbf{a}}(i)\right)}=\boldsymbol{\Gamma} .
$$

Substituting Eq. 6.65) and 6.66) in to Eq. (6.64) yields:

$$
\begin{aligned}
& \Delta V(i)=\mathbf{s}(i)^{\top}(\mathbf{s}(i+1)-\mathbf{s}(i)) \\
& +\tilde{\mathbf{a}}(i)^{\top} \boldsymbol{\Gamma}(\tilde{\mathbf{a}}(i+1)-\tilde{\mathbf{a}}(i)) \\
& +\frac{1}{2}\left(\Delta \mathbf{s}(i)^{\top} \Delta \mathbf{s}(i)+\Delta \tilde{\mathbf{a}}(i)^{\top} \boldsymbol{\Gamma} \Delta \tilde{\mathbf{a}}(i)\right) .
\end{aligned}
$$

A new function, $O(i)$ is defined based on the second order terms in Eq. (6.67):

$$
O(i)=\frac{1}{2}\left(\Delta \mathbf{s}(i)^{\top} \Delta \mathbf{s}(i)+\Delta \tilde{\mathbf{a}}(i)^{\top} \boldsymbol{\Gamma} \Delta \tilde{\mathbf{a}}(i)\right) .
$$

According to Eq. 6.61) and 6.68), Eq. 6.67) can be simplified as follows: 


$$
\begin{aligned}
\Delta V(i) & =\mathbf{s}(i)^{\top}(-\boldsymbol{\beta} \mathbf{s}(i)+T \mathbf{F} \tilde{\mathbf{a}}(i)-\mathbf{s}(i)) \\
& +\tilde{\mathbf{a}}(i)^{\top} \boldsymbol{\Gamma}(\tilde{\mathbf{a}}(i+1)-\tilde{\mathbf{a}}(i))+O(i) .
\end{aligned}
$$

By re-arranging Eq. (6.69), we obtain:

$$
\begin{gathered}
\Delta V(i)=-\mathbf{s}(i)^{\top}(\boldsymbol{\beta}+\mathbf{I}) \mathbf{s}(i) \\
+\tilde{\mathbf{a}}(i)^{\top} \boldsymbol{\Gamma}\left(T \boldsymbol{\Gamma}^{-1} \mathbf{F}^{\top} \mathbf{s}(i)-\hat{\mathbf{a}}(i+1)+\hat{\mathbf{a}}(i)\right)+O(i) .
\end{gathered}
$$

If the following equation, as the adaptation law, is used to update the estimation of the unknown parameters vector $(\hat{\mathbf{a}}(i))$ :

$$
\hat{\mathbf{a}}(i+1)=\hat{\mathbf{a}}(i)+T(\boldsymbol{\Gamma} \boldsymbol{\Gamma})^{-1} \mathbf{F}^{\top} \mathbf{s}(i),
$$

then, Eq. 6.70 becomes:

$$
\Delta V(i)=-\mathbf{s}(i)^{\top}(\boldsymbol{\beta}+\mathbf{I}) \mathbf{s}(i)+O(i) .
$$

One can easily find the analogous structure between MIMO (Eq. (6.71) ) and SISO (Eq. 6.25) adaptation laws. Next, by expanding the second order term $(O(i))$ with respect to Eq. 6.68, and assuming that the model uncertainties have been removed by the adaptation law (Eq. 6.71) ), Eq. 6.72 becomes:

$$
\Delta V(i)=-\mathbf{S}(i)^{\top}(\boldsymbol{\beta}+\mathbf{I}) \mathbf{s}(i)+\frac{1}{2} \mathbf{S}(i)^{\top}(\boldsymbol{\beta}+\mathbf{I})^{\top}(\boldsymbol{\beta}+\mathbf{I}) \mathbf{s}(i)
$$

Finally, assuming that $\beta$ is symmetric, Eq. 6.73 can be simplified as: 


$$
\Delta V(i)=-\frac{1}{2} \mathbf{s}(i)^{\top}(\mathbf{I}-\boldsymbol{\beta} \boldsymbol{\beta}) \mathbf{s}(i) .
$$

$\Delta V$ is negative semi-definite if eigenvalues of $\boldsymbol{\beta}$ lie within the unit circle [59].

Although the Lyapunov function in Eq. 6.62) guarantees the finite-time zero convergence of the first order sliding vector (s), and intuitively gives the adaptation law to remove the uncertainty in the model, still it is required to prove the asymptotic convergence of the second order sliding vector $(\boldsymbol{\xi})$ to zero. To this end, first it is assumed that the uncertainty in the model is removed permanently by incorporating the adaptation rule from Eq. (6.71). Validity of this assumption will be testified in Section 6.4.3. Next, the system switches to another Lyapunov function $\left(V^{*}\right.$, proposed in Eq. (6.75) ), which covers the time interval after the completion of the adaptation period.

The idea of multiple Lyapunov functions has been studied for switched and hybrid systems in the literature [106, 107, 108]. The study in reference [106] presents a stability analysis based on multiple Lyapunov functions for discontinuous systems, where the dynamic of the system $(f)$ switches between multiple functions that are globally Lipschitz continuous. Here, it is assumed that the dynamics hold the Lipschitz condition, but it does not switch. The sufficient condition, to show the stability of the system in the sense of Lyapunov, is to show that at the switching sequence, both of the Lyapunov functions $\left(V, V^{*}\right)$ are Lyapunov-like [106]. For our case, in 
which $V$ switches just once, the Lyapunov functions are Lyapunov-like when the difference functions are at least negative semi-definite [106]. As shown before, for $V$ from Eq. (6.62), the negative semi-definite condition for $\Delta V$ holds. Thus, it is required that the new candidate function $\left(V^{*}\right)$ is also Lyapunov-like. The new Lyapunov function is the extended version of the Lyapunov function in Eq. (6.62), which not only preserves the nature of the problem, but also takes into account the finite-time zero convergence conditions of the second order sliding vector $(\boldsymbol{\xi})$, which is required to prove the asymptotic stability of the second order MIMO DSMC.

We define the new Lyapunov function $\left(V^{*}\right)$ as follows:

$$
V^{*}(i)=\frac{1}{2}\left(\mathbf{s}(i+1)^{\top} \mathbf{s}(i+1)+\mathbf{s}(i)^{\top} \boldsymbol{\beta} \mathbf{s}(i)\right) .
$$

By using the Taylor series expansion, implementing the values for the first and second order partial derivatives of $V^{*}$ with respect to $\mathbf{s}(i)$ and $\mathbf{s}(i+1)$, and knowing that all the higher order partial derivatives and also second order cross derivative are zero, the Lyapunov difference function becomes:

$$
\begin{aligned}
\Delta V^{*}(i) & =\mathbf{s}(i)^{\top} \boldsymbol{\beta}(\mathbf{s}(i+1)-\mathbf{s}(i)) \\
& +\mathbf{s}(i+1)^{\top}(\mathbf{s}(i+2)-\mathbf{s}(i+1)) \\
& +\frac{1}{2}\left(\Delta \mathbf{s}(i)^{\top} \boldsymbol{\beta} \Delta \mathbf{s}(i)\right. \\
& \left.+\Delta \mathbf{s}(i+1)^{\top} \Delta \mathbf{s}(i+1)\right) .
\end{aligned}
$$

According to the earlier assumption, upon removal of the uncertainties in the model, 
Eq. 6.61 becomes $\boldsymbol{\xi}(i)=\mathbf{0}$. Thus, Eq. 6.76 can be simplified as follows:

$$
\begin{aligned}
\Delta V^{*}(i) & =-\mathbf{s}(i)^{\top} \boldsymbol{\beta}(\boldsymbol{\beta}+\mathbf{I}) \mathbf{s}(i) \\
& -\mathbf{s}(i+1)^{\top}(\boldsymbol{\beta}+\mathbf{I}) \mathbf{s}(i+1) \\
& +\frac{1}{2}\left(\Delta \mathbf{s}(i)^{\top} \boldsymbol{\beta} \Delta \mathbf{s}(i)\right. \\
& \left.+\Delta \mathbf{s}(i+1)^{\top} \Delta \mathbf{s}(i+1)\right) .
\end{aligned}
$$

In a similar manner to the SISO system, it can be shown that upon applying the equivalent control input of the MIMO second order DSMC (Eq. (6.60) ) along with the vector of the switching functions $\left(\left|\mu_{u_{i}}\right| \operatorname{sat}\left(\xi_{i}\right)\right)$ on the MIMO form of Gao's reaching law [109, 110], and assuming the removal of the model uncertainties by using Eq. 6.71, we obtain:

$$
\mathbf{s}(i+2) \approx \boldsymbol{\beta}^{2} \mathbf{s}(i)
$$

where the input of the second order MIMO DSMC is [109, 110]:

$$
\mathbf{u}=\mathbf{u}_{e q}(i)-\left[\begin{array}{c}
\left|\mu_{u_{1}}\right| \operatorname{sat}\left(\xi_{1}(i-1)\right) \\
\vdots \\
\left|\mu_{u_{r}}\right| \operatorname{sat}\left(\xi_{i}(i-1)\right)
\end{array}\right]
$$

in which $\mu_{u_{i}}, i=0: r$ is the predicted uncertainties on the corresponding control signal. Next, by substituting Eq. (6.78) into Eq. 6.77), we have:

$$
\Delta V^{*}(i)=-\mathbf{s}^{\top} \boldsymbol{\beta}\left(-\boldsymbol{\beta}^{3}-\boldsymbol{\beta}^{2}+\boldsymbol{\beta}-\mathbf{I}\right) \mathbf{s}(i),
$$


which also has a similar structure to Eq. 6.42). $\Delta V^{*}$ in Eq. 6.80, is negative semidefinite in $\mathbf{s}(i)$ when $\boldsymbol{\beta}$ is chosen such that its eigenvalues lie within the unit circle. More importantly, due to the analogous structure between MIMO and SISO adaptive second order DSMCs, the asymptotic stability of the MIMO controller can be proved by invoking the new Invariance Principle [94, 104] and noting that the higher order Lyapunov difference functions are zero, if and only if $\mathbf{s}=\mathbf{0}[62]$.

\subsection{Nonlinear Case Study: Engine Control}

In this section, the proposed adaptive second order DSMC is designed for the physicsbased spark ignition (SI) combustion engine model during transient cold start period (Eq. (4.28)-4.31) ). Four states of the model and corresponding nonlinear dynamics in the state-space structure are as follows [86]:

$$
\begin{aligned}
& {\left[\begin{array}{c}
T_{\text {exh }}(i+1) \\
\dot{m}_{f}(i+1) \\
m_{a}(i+1) \\
\omega_{e}(i+1)
\end{array}\right]=\left[\begin{array}{c}
T_{e x h}(i) \\
\dot{m}_{f}(i) \\
m_{a}(i) \\
\omega_{e}(i)
\end{array}\right]+T\left(\left[\begin{array}{cccc}
f_{T_{e x h}}(i) & 0 & 0 & 0 \\
0 & f_{\dot{m}_{f}}(i) & 0 & 0 \\
0 & 0 & f_{m_{a}}(i) & 0 \\
0 & 0 & 0 & f_{\omega_{e}}(i)
\end{array}\right]\left[\begin{array}{c}
\alpha_{T_{e x h}} \\
\alpha_{\dot{m}_{f}} \\
\alpha_{m_{a}} \\
\alpha_{\omega_{e}}
\end{array}\right]\right.} \\
& \left.+\left[\begin{array}{cccc}
g_{T_{e x h}}(i) & 0 & 0 & 0 \\
0 & g_{\dot{m}_{f}}(i) & 0 & 0 \\
0 & 0 & g_{m_{a}}(i) & 0 \\
0 & 0 & 0 & g_{\omega_{e}}(i)
\end{array}\right]\left[\begin{array}{c}
\Delta(i) \\
\dot{m}_{f, c}(i) \\
\dot{m}_{a i}(i) \\
m_{a, d}(i)
\end{array}\right]\right)
\end{aligned}
$$


where $\mathbf{F}=\operatorname{diag}\left[f_{T_{e x h}}, f_{\dot{m}_{f}}, f_{\omega_{e}}, f_{m_{a}}\right]$, and:

$$
\begin{gathered}
f_{T_{e x h}}=\frac{1}{\tau_{e}}\left[600 A F I-T_{e x h}\right] \\
f_{\dot{m}_{f}}=-\frac{1}{\tau_{f}} \dot{m}_{f}(i) \\
f_{\omega_{e}}=-\frac{1}{J}\left(0.4 \omega_{e}(i)+100\right) \\
f_{m_{a}}=-\dot{m}_{a o} .
\end{gathered}
$$

Also $\Upsilon=\operatorname{diag}\left[g_{T_{e x h}}, g_{\dot{m}_{f}}, g_{\omega_{e}}, g_{m_{a}}\right]$, and:

$$
\begin{aligned}
& g_{T_{e x h}}=\frac{7.5}{\tau_{e}}, \quad g_{\dot{m}_{f}}=\frac{1}{\tau_{f}} \\
& g_{\omega_{e}}=\frac{30000}{J}, \quad g_{m_{a}}=1 .
\end{aligned}
$$

The sliding vector is:

$$
\mathbf{s}(k)=\left[\begin{array}{c}
s_{1}(i) \\
s_{2}(i) \\
s_{3}(i) \\
s_{4}(i)
\end{array}\right]=\left[\begin{array}{c}
T_{e x h}(i)-T_{e x h, d}(i) \\
A F R(i)-A F R_{d}(i) \\
m_{a}(i)-m_{a, d}(i) \\
\omega_{e}(i)-\omega_{e, d}(i)
\end{array}\right] .
$$

The equivalent control input vector $\left(\mathbf{u}_{e q}\right)$ of the baseline second order DSMC for the

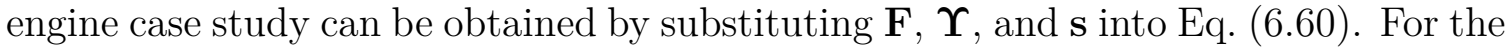
SISO DSMC, $\boldsymbol{\beta}$ is chosen to be diagonal, while for the MIMO controller, the dynamic coupling is included via the off-diagonal element of $\boldsymbol{\beta}$. For the second order DSMC with $A D C$ uncertainties, according to Eq. (6.79), the predicted ADC uncertainties 
are incorporated into the DSMC structure through the switching control input $\left(\mathbf{u}_{s w}\right)$ gains. For the engine case study, the gains of the switching control input $\left(\mu_{\Delta}, \mu_{\dot{m}_{f c}}\right.$, $\left.\mu_{\dot{m}_{a i}}, \mu_{m_{a, d}}\right)$ are estimated online using the mechanism previously shown in Figure 4.2 .

\subsubsection{Handling Implementation Imprecisions}

In order to demonstrate the robustness characteristics of the second order DSMC compared to the first order controller in handling ADC uncertainties, first we assume that the engine model is ideal and there is no uncertainty in the modeled dynamics $\left(\alpha_{T_{e x h}}=\alpha_{\dot{m}_{f}}=\alpha_{\omega_{e}}=\alpha_{m_{a}}=1\right)$. Figure 6.1 shows the desired trajectories $\left(A F R, T_{\text {exh }}\right.$, and

engine speed) tracking results, using the first and second order DSMCs for sampling times of $20 \mathrm{~ms}$ and $80 \mathrm{~ms}$, and quantization level of 16-bit and 10-bit, respectively. The mean tracking errors for both controllers are listed in Table 6.1. It can be observed from Figure 6.1 and Table 6.1 that, when the signals at the controller I/O are sampled every $20 \mathrm{~ms}$, both first and second order baseline DSMCs illustrate smooth and acceptable tracking performances, while the second order controller is more accurate by up to $67 \%$ in terms of the tracking errors.

Upon increasing the sampling rate from $20 \mathrm{~ms}$ to $80 \mathrm{~ms}$, and changing the ADC quantization level from 16-bit to 10-bit, the first order DSMC performance degrades significantly. On the other side, the second order DSMC still presents accurate tracking results. By comparing the first and second order DSMC results at $T=80 \mathrm{~ms}$ 
Table 6.1

Mean $(\bar{e})$ of Tracking Errors. Values Inside the Parentheses Show the Resulting Improvement from the Second Order DSMC Compared to the First Order DSMC.

\begin{tabular}{|c|c|c|c|c|}
\hline & \multicolumn{2}{|c|}{$\overline{\bar{e}}(T=20 \mathrm{~ms}, 16-b i t)$} & \multicolumn{2}{|c|}{$\overline{\bar{e}}(T=80 \mathrm{~ms}, 10-b i t)$} \\
\hline & $1^{s t}$-Order & $2^{n d}$-Order & $1^{\text {st }}$-Order & $2^{n d}$-Order \\
\hline & DSMC & DSMC & DSMC & DSMC \\
\hline & Reference & & Reference & \\
\hline AFR & 0.03 & 0.01 & 0.28 & 0.03 \\
\hline$[-]$ & & $(-66.67 \%)$ & & $(-89.29 \%)$ \\
\hline$T_{e x h}$ & 0.2 & 0.1 & 4.0 & 0.4 \\
\hline$\left[{ }^{\circ} \mathrm{C}\right]$ & & $(-50 \%)$ & & $(-90.0 \%)$ \\
\hline$N$ & 0.1 & 0.1 & 13.8 & 0.9 \\
\hline$[\mathrm{RPM}]$ & & $(\approx 0 \%)$ & & $(-93.5 \%)$ \\
\hline
\end{tabular}

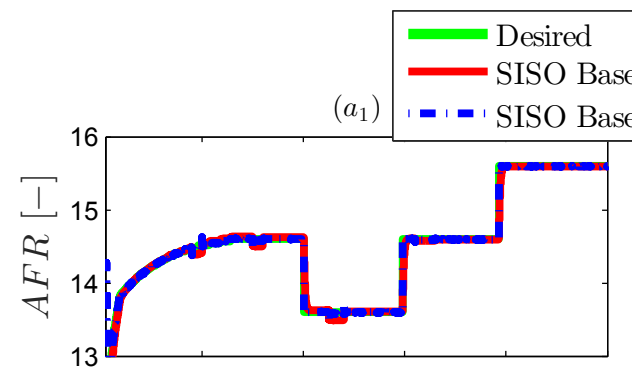

$\left(b_{1}\right)$
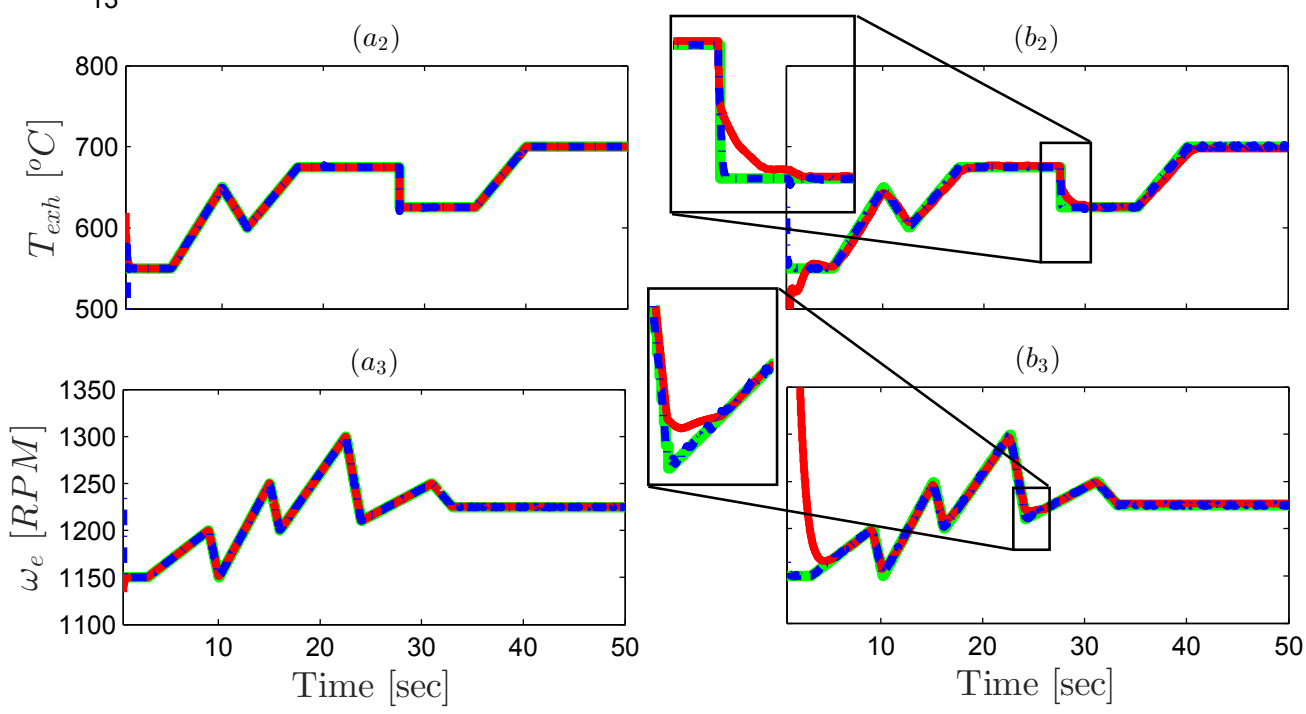

Figure 6.1: Engine tracking results by the first and second order SISO DSMCs for $(a) T=20 \mathrm{~ms}$, quantization level=16-bit, and (b) $T=80 \mathrm{~ms}$, quantization level=10-bit. 
and quantization level of 10-bit, it can be concluded that the proposed second order DSMC offers higher robustness against ADC uncertainties, and outperforms the first order controller by up to $90 \%$ in terms of the mean tracking errors.

For the SI engine case study, $A F R$ controller is the most uncertainty-sensitive controller, in comparison with the engine speed and exhaust gas temperature controllers [2, 3]. Figure 6.2 shows the tracking results of the first (SISO) and second (SISO and MIMO) order DSMCs under a relatively large sampling rate of $200 \mathrm{~ms}$, which causes significant uncertainty at the controller I/O. As shown in Figure 6.2, the first order DSMC fails to track all the desired trajectories under these extreme ADC uncertainties, but the SISO second order DSMC shows acceptable tracking performances.

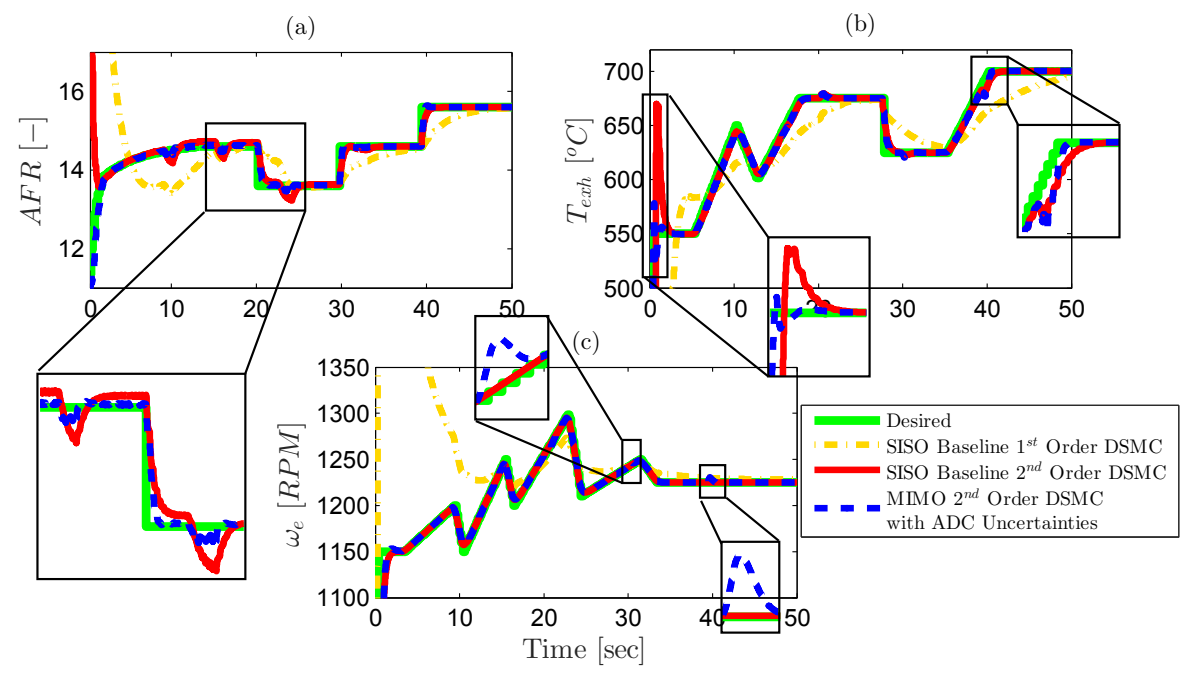

Figure 6.2: Results of desired trajectories tracking from SISO Baseline $1^{\text {st }}$ and $2^{\text {nd }}$ order DSMCs, and MIMO $2^{\text {nd }}$ order DSMC with predicted ADC uncertainties $(T=200 \mathrm{~ms}$, quantization level=16-bit $)$. 
Figure 6.2 a shows that among the three SISO second order controllers, the AFR controller is deviated more upon increasing the sampling rate. This deviation from the desired AFR trajectories is larger when there is a change in the desired engine speed trajectory (Figure 6.2 -c). The link between engine speed and AFR controllers can be traced in the strong coupling between AFR and rotational dynamics via the intake air mass flow term $\left(\dot{m}_{a o}\right)$ :

$$
\begin{gathered}
A F R=\frac{\dot{m}_{a o}}{\dot{m}_{f}} \\
m_{a}(i+1)=m_{a}(i)+T\left(\dot{m}_{a i}(i)-\dot{m}_{a o}(i)\right) .
\end{gathered}
$$

Moreover, Figure 6.2 b shows that the changes in desired engine speed profile have an effect on $T_{\text {exh }}$ controller performance. Similar to the AFR controller, the link between $T_{\text {exh }}$ and engine speed controllers is the exhaust gas temperature time constant $\left(\tau_{e}\right)$ which is calculated with respect to the engine speed:

$$
\tau_{e}=\frac{2 \pi}{\omega_{e}}
$$

The coupling within the engine dynamics can be represented in the MIMO DSMC design via $\boldsymbol{\beta}$ matrix. The diagonal elements of $\boldsymbol{\beta}$ are the same as the SISO controller, while the off-diagonal element represents the coupling between various sliding variables. According to Eq. 6.85) and (6.86), $\boldsymbol{\beta}$ is defined to present the coupling between AFR, $T_{e x h}$, and engine speed controllers. Additionally, the engine speed and air mass flow dynamics are inherently coupled because of the synthetic $m_{a, d}$ control 
input which is the input to the engine speed controller, and the reference trajectory for the air mass flow controller. Previously, the results in [111, 112] showed that allowing $\dot{m}_{f, c}$ (used to regulate $A F R$ ) to depend upon the cam phasing (rotational dynamics) leads to smaller transients in $A F R$ tracking results. With a similar trend to [111, 112], here, Figure 6.2 a shows that by utilizing the MIMO controller with predicted ADC uncertainties, not only the AFR tracking error decreases (by 46\%), but also the effects of the engine speed trajectory variation on the AFR tracking become smaller.

In a similar manner to $A F R$ controller, by utilizing the MIMO second order controller

for $T_{\text {exh }}$, the desired $T_{\text {exh }}$ tracking performance improves slightly (by 11\%) compared to the SISO second order DSMC, and the large overshoot at the beginning of the simulation will be removed. On the other side, by looking into Figure 6.2 -c, it can be seen that changing the engine speed controller to MIMO structure has almost no significant effect on the tracking performance. As highlighted in Figure 6.2-c, the MIMO controller results in small spikes, which occur when the desired AFR trajectory has a sudden change (Figure 6.2 a).

\subsubsection{External Disturbance Rejection}

Another interesting feature of the second order DSMC, in comparison with the first order controller, is its unknown external disturbance rejection characteristics. To 
illustrate this feature, here the DSMC response to external disturbing torque load is investigated. A physical example of this disturbing torque is when a driver turns on a car's air conditioning unit. This acts as disturbance for engine speed/torque controller. The disturbing torque directly impacts the rotational dynamics of the engine. However, since the AFR and $T_{\text {exh }}$ controllers are linked to engine speed controller, any changes in the engine speed dynamics can affect $A F R$ and, with a lower degree, $T_{\text {exh }}$ controllers performance accordingly [3]. Figure 6.3 shows the profile of the disturbing torque $\left(T_{d}=5 \mathrm{Nm}\right)$ which is applied for $10 \mathrm{~s}$ during the simulation time. Figure 6.4 shows the comparison between the baseline first and second order SISO DSMCs under $20 \mathrm{~ms}$ of sampling time and quantization level of 16-bit. As expected, the disturbing torque affects AFR and speed tracking results. It can be seen from Figure 6.4-c that both first and second order DSMCs can reject the disturbance, however, the second order controller reacts faster to the disturbance, and provides better tracking performance. By looking into Figure 6.4 a, it can be observed that although the second order DSMC rejects the effects of the external disturbance very fast, it comes at the cost of slightly higher overshoots in the desired AFR trackj

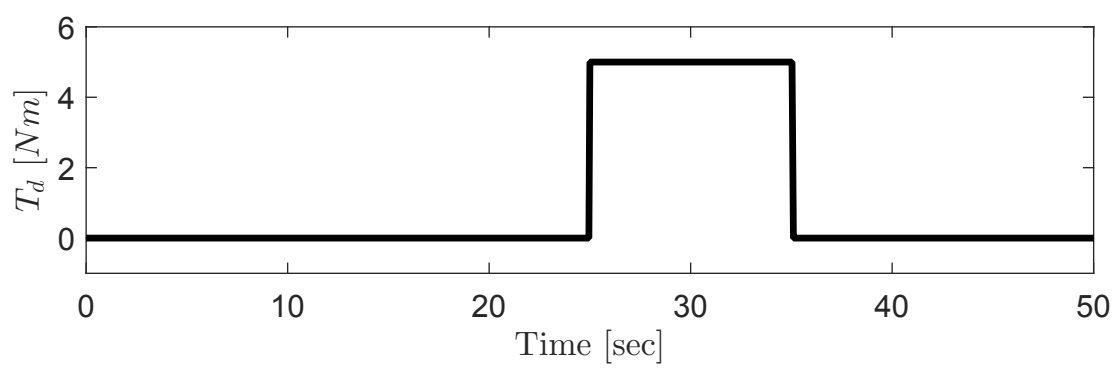

Figure 6.3: Disturbing load torque profile. 


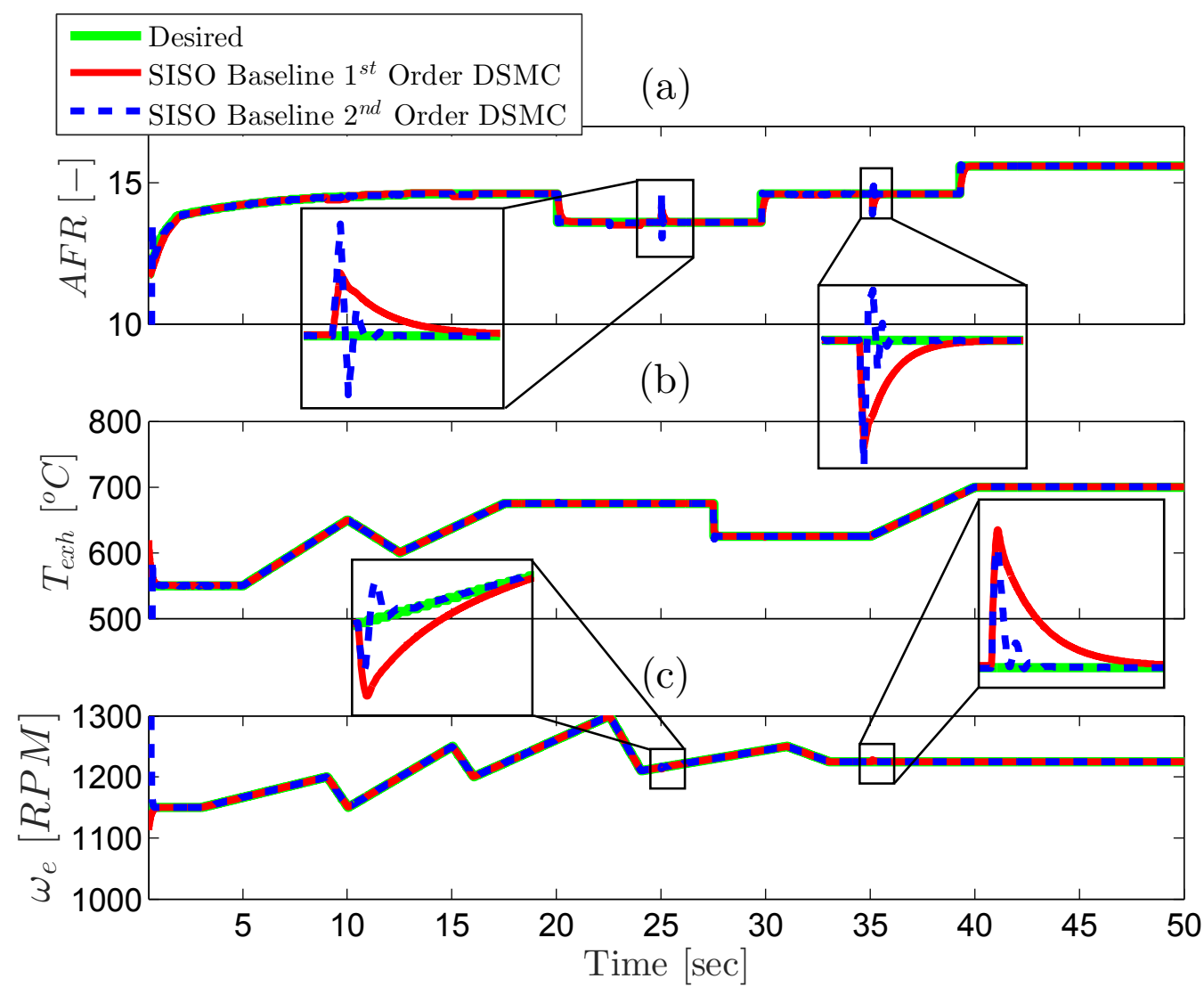

Figure 6.4: Disturbance rejection performance of the SISO baseline first and second order DSMCs for the disturbing torque profile shown in Figure $6.3(T=20 \mathrm{~ms}$, quantization level=16-bit $)$.

The disturbing torque load rejection performance is investigated at a higher sampling rate $(80 \mathrm{~ms})$ with the same external torque load profile shown in Figure 6.3. This time, the baseline MIMO second order DSMC is also simulated in addition to the SISO first and second order baseline controllers. Their tracking results are shown in Figure 6.5, where it can be seen that both SISO and MIMO second order DSMCs show better and faster tracking results compared to the first order controller. While both SISO and MIMO second order engine speed DSMCs show very fast reactions to the disturbing torque (Figure 6.5 c), the MIMO controller makes the $A F R$ (Figure 6.5 a) and $T_{e x h}$ 


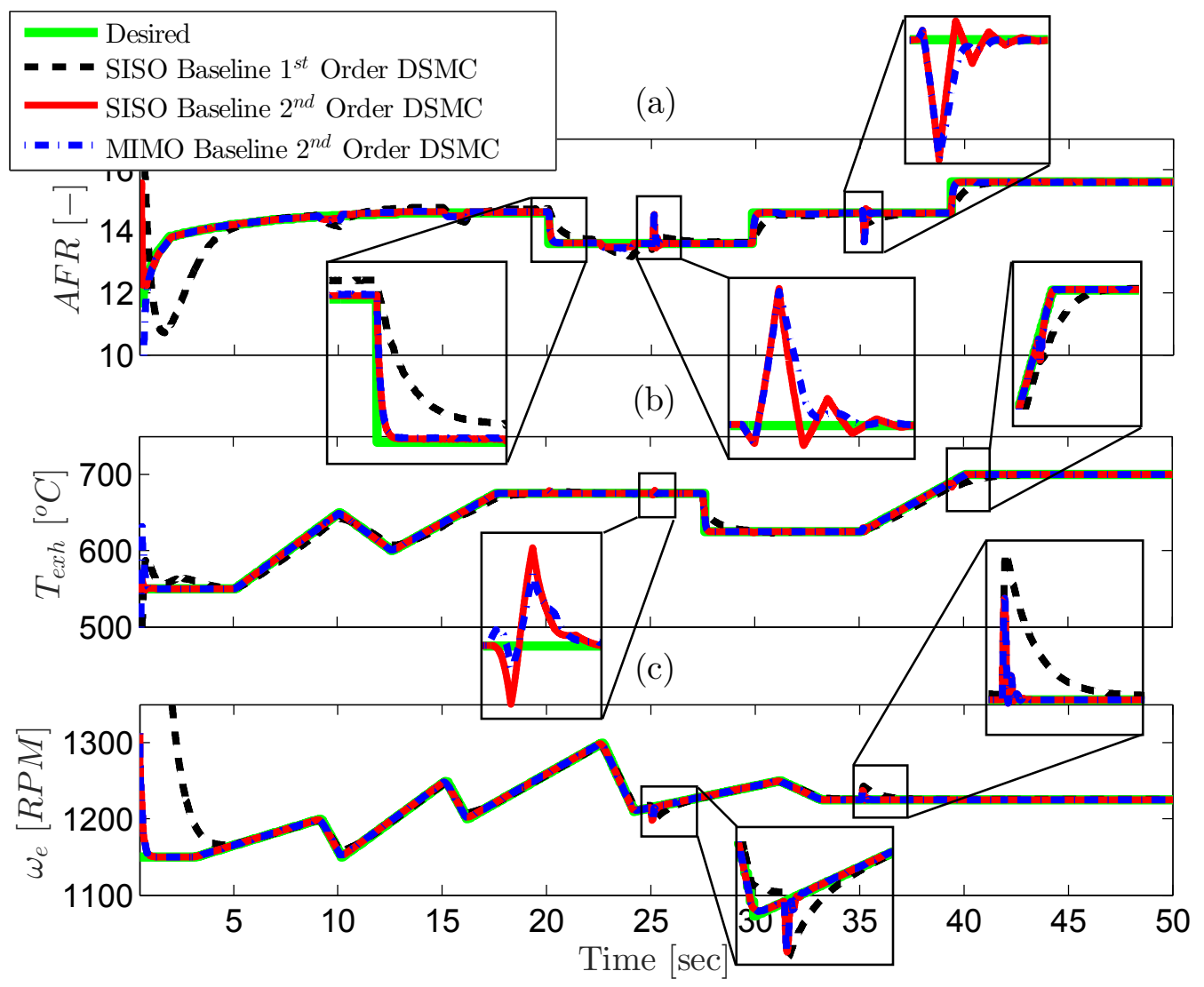

Figure 6.5: Disturbance rejection performance of the SISO and MIMO baseline first and second order DSMCs for the disturbing torque profile shown in Figure $6.3(T=80 \mathrm{~ms}$, quantization level=16-bit).

(Figure 6.5-b) controllers' response to the disturbance become more smooth compared to the SISO second order DSMC. This is because the coupling among different engine dynamics is reflected into the controller structure via the MIMO design. Therefore, when there is a disturbance on one of the controllers, other controllers can react accordingly to minimize the impact of the disturbance on the overall controller's performance.

The comparison results between the SISO and MIMO second order DSMCs from 
Figure 6.2 and Figure 6.5 are consistent with our previous observation in [62], where the MIMO first order DSMC is evaluated. Overall, by using the MIMO structure, better tracking performance for $A F R$ and $T_{e x h}$ controllers can be achieved. However, the performance of the engine speed controller leans toward a minimally coupled structure.

\subsubsection{Real-Time Engine $2^{\text {nd }}$ Order DSMC Verification}

In the next step, the performance of the proposed adaptation mechanism for the SISO/MIMO second order DSMC is investigated under up to $50 \%$ multiplicative uncertainty within the engine model. The uncertainty withing the engine model was studied with details in Section 5.3 in Chapter 5. Since the adaptation laws for the first and second order DSMCs have the same structures, Eq. (5.21), 5.24, (5.27), and $(5.32)$ are recalled as the adaptation laws for the second order DSMC. In this section, the performance of both SISO and MIMO adaptive second order DSMCs will be evaluated by testing the controller software on a real ECU in a PIL setup (Figure 3.6) to verify the performances of the adaptive DSMC in real-time.

The model of the engine plant is built into the PXI processor. The output of the PXI processor is the controller feedback signal from the plant. Using embedded ADC units, the feedback signal is sampled and quantized at $80 \mathrm{~ms}$ and 16-bit, respectively. On the other side, the adaptive second order DSMC logic along with the adaptation 
and uncertainty prediction mechanisms are implemented into the MABX, which is the main ECU. The output of the MABX is the control signal which is set to be updated at every $80 \mathrm{~ms}$.

Figure 6.6 shows the results of four unknown multiplicative parameters estimation from SISO and MIMO second order DSMCs with predicted ADC uncertainties. It can be seen that under up to $50 \%$ uncertainty on each of the engine model's dynamics, by using the proposed adaptation mechanism, the unknown terms converge to their nominal values, "1", in less than 4 sec. Moreover, the MIMO controller shows faster convergence for $\alpha_{T_{e x h}}$ and $\alpha_{\dot{m}_{f}}$, while for $\alpha_{m_{a}}$ and $\alpha_{\omega_{e}}$, SISO and MIMO controllers show similar convergence behaviour. Additionally, it can be observed that for all cases, despite the variation in the desired trajectories, after completion of the adaptation period, the uncertainties in the models are removed permanently. Finally, the PIL testing results show that the proposed adaptation mechanism is able to operate in real-time since it is computationally efficient. The adaptation time is significantly reduced if shorter sampling time is applied.

Figure 6.7 shows the desired trajectory tracking results form the non-adaptive, and SISO/MIMO second order DSMCs. First of all, it can be seen that in the absence of the adaptation mechanism, due to the large uncertainties in the plant model $(50 \%)$, the non-adaptive controller fails to track the desired trajectories for all the cases. Upon activation of the adaptation mechanism, it can be seen that after completion 
(a)

(b)

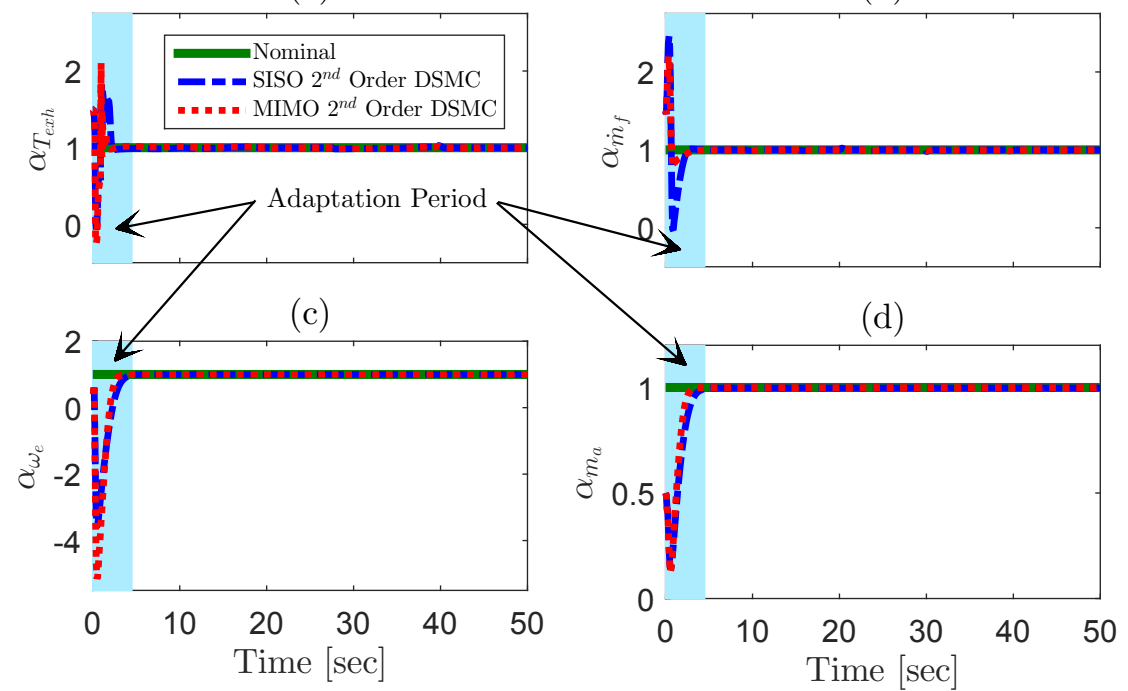

Figure 6.6: Results of unknown multiplicative parameters convergences $(T=80 \mathrm{~ms}$, quantization level=16-bit $)$.

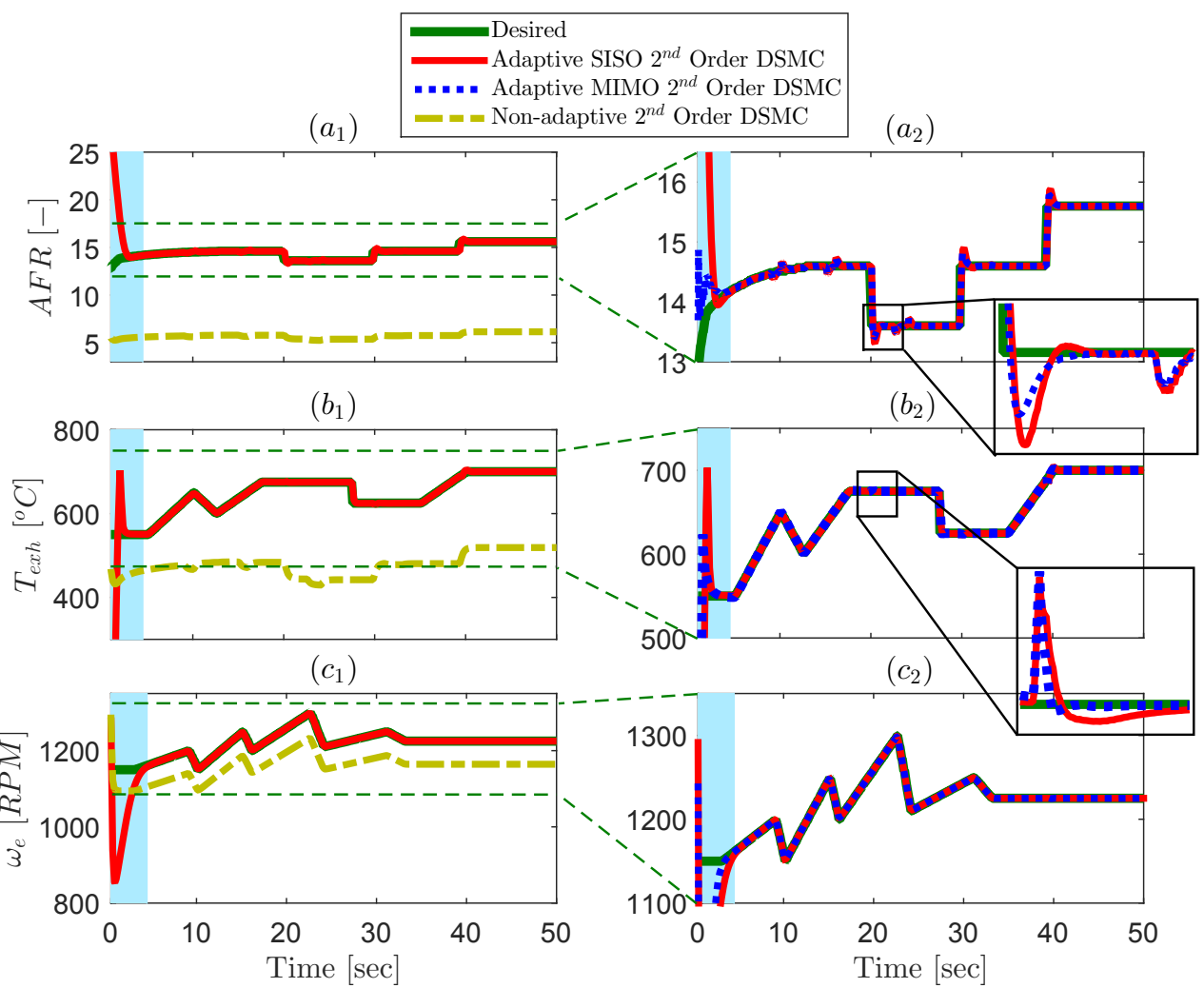

Figure 6.7: Results of engine control under model uncertainties: (a) AFR, (b) exhaust gas temperature, and (c) engine speed ( $T=80 \mathrm{~ms}$, quantization level=16-bit). 
of the adaptation period, both SISO and MIMO adaptive second order DSMCs with predicted ADC uncertainties provide accurate tracking performances. By comparing the non-adaptive and adaptive DSMCs it is revealed that the adaptation mechanism is able to remove the uncertainties in the model by more than $95 \%$, that consequently results in more than $90 \%$ improvement in the controller tracking performance.

Figure 6.7 shows that the MIMO and SISO second order DSMCs have similar tracking behavior for the engine speed tracking. However, by using the MIMO structure, the tracking performance for $A F R$ and $T_{\text {exh }}$ controllers can be improved by $43 \%$, and $33 \%$, respectively. These improvements are more significant during the adaptation period, and also at those points where there are sudden changes in the desired engine speed profile. The latter observations can be explained based on the engine dynamics, in which the engine speed loop acts as a disturbance to $A F R$ and $T_{\text {exh }}$ (Eq. 6.85) and (6.86)). By using the MIMO controller, $\dot{m}_{f c}$ and $\Delta$, which are used respectively to regulate $A F R$ and $T_{e x h}$, can be configured to depend upon the engine speed dynamics. Linking the control input of the $A F R$ and $T_{\text {exh }}$ controllers to the rotational dynamics allows for better $A F R$ and $T_{\text {exh }}$ tracking performances during the engine speed transients. 


\subsection{Second Order Adaptive DSMC for Uncertain Linear Systems}

The second order DSMC control input is calculated by solving Eq. (6.16] [99]. Applying Eq. 6.16 to the linear system in Eq. (5.35), in the absence of model and ADC uncertainties, results in the following control input $(\mathbf{U})$ for the second order DSMC:

$$
\mathbf{U}(i)=\mathbf{B}^{-1}\left(\frac{1}{T}\left[(\boldsymbol{\beta}+\mathbf{I}) \mathbf{X}(i)-\boldsymbol{\beta} \mathbf{X}_{d}(i)+\mathbf{X}_{d}(i+1)\right]-\mathbf{A X}(i)\right)
$$

The control input of the baseline second order DSMC (Eq. 6.87) can be modified $\left(\mathbf{U}^{\text {mod }}\right)$ against sampling and quantization imprecisions by inclusion of the propagated $\operatorname{ADC}$ uncertainties $\left(\mu_{\mathbf{U}}\right)[8]$ :

$$
\mathbf{U}^{\text {mod }}(i)=\mathbf{U}(i)-\left|\mu_{\mathbf{U}}(i)\right| \times \operatorname{sat}(\boldsymbol{\xi}(i-1))
$$

where $\mu_{\mathbf{U}}$ is calculated according to Eq. (5.40). By applying Eq. (6.88) to the linear system (Eq. (5.35) ) in the presence of the unknown multiplicative (no additive un-

certainty term is considered), the vector $(\boldsymbol{\xi})$ of the second order sliding variables $(\xi)$ becomes:

$$
\boldsymbol{\xi}_{r \times 1}(i)=T\left[\begin{array}{ccc}
\tilde{\alpha}_{11} a_{11} & \ldots & \tilde{\alpha}_{1 r} a_{1 r} \\
\tilde{\alpha}_{21} a_{21} & \ldots & \tilde{\alpha}_{2 r} a_{2 r} \\
\vdots & \ddots & \vdots \\
\tilde{\alpha}_{r 1} a_{r 1} & \ldots & \tilde{\alpha}_{r r} a_{r r}
\end{array}\right] \mathbf{X}_{r \times 1}(i)
$$

The multiplicative uncertainty within the linear model is removed $(\hat{\alpha} \rightarrow 0: \hat{\alpha} \rightarrow \alpha)$ 
by utilizing Eq. (5.44) from Chapter 5 .

\subsubsection{Linear Case Study: DC Motor Speed Control}

In a similar manner to the first order adaptive DSMC design for the DC motor case study (Section 5.4.1), the control inputs of the adaptive second order SISO DSMC with predicted ADC uncertainties are as follows:

$$
\begin{array}{r}
\mathcal{I}_{d, \text { adaptive }}^{\text {mod }}(i)=\frac{J}{k_{m}}\left(\frac{1}{T}\left[-\beta_{1}\left(s_{1}(i)\right)+\theta_{d}(i+1)-\theta(i)\right]\right. \\
\left.+\left[\hat{\alpha}_{11} \frac{k_{f}}{J}\right] \theta(i)-\frac{1}{J} \Gamma\right)-\left|\mu_{\mathcal{I}_{d}}(i)\right| \operatorname{sat}\left(\xi_{1}(i-1)\right) \\
V_{\text {adaptive }}^{\text {mod }}(i)=L\left(\frac{1}{T}\left[-\beta_{2}\left(s_{2}(i)\right)+\mathcal{I}_{d}(i+1)-\mathcal{I}(i)\right]\right. \\
\left.+\left[\hat{\alpha}_{21} \frac{k_{b}}{L}\right] \theta(i)+\left[\hat{\alpha}_{22} \frac{R}{L}\right] \mathcal{I}(i)\right)-\left|\mu_{V}(i)\right| \operatorname{sat}\left(\xi_{2}(i)\right)
\end{array}
$$

The SISO second order DSMC from Eq. (6.90) and (6.91) can be converted into a MIMO structure via the second order sliding mode tuning gains $(\beta)$, in which the off-diagonal elements of $\boldsymbol{\beta}$ are chosen to be non-zero to reflect the coupling between the states of the DC motor model in the controller structure. In the absence of model uncertainties $\left(\alpha_{p q}=1\right)$, Figure 6.8 shows the comparison between the SISO first, and SISO/MIMO second order DSMCs for different sampling rates. Shannon's sampling theorem criteria states that the sampling frequency must be at least twice the maximum frequency of the measured analog signal [113]. As long as the Shannon's sampling theorem is satisfied, increasing the sampling time helps to reduce the computation cost. Although at lower sampling rates (e.g. $200 \mathrm{~ms}$ ) all the controllers 
show similar performances, by increasing the sampling rate to $800 \mathrm{~ms}$, the higher robustness characteristics of both SISO and MIMO second order DSMCs in comparison with the first order controller is revealed. The comparison results show that the SISO second order DSMC is improving the tracking errors by $69 \%$ on average for different sampling rates, compared to the first order controller. Moreover, it can be seen that the MIMO second order controller is barely affected by the sampling time increase, and this illustrates its strone robustness a.gainst ADC uncertainties.

(a) SISO First Order DSMC

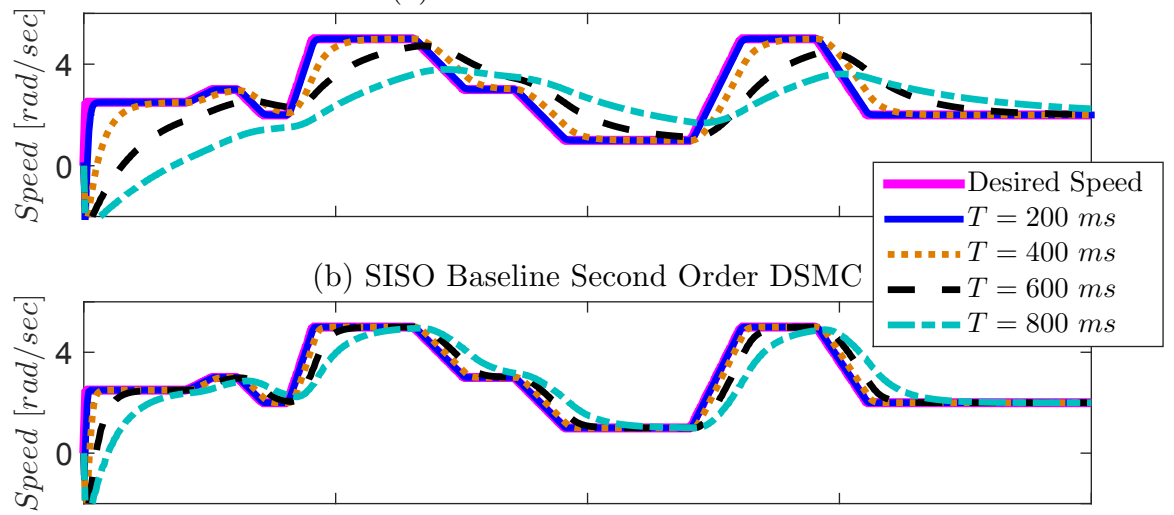

(c) MIMO Baseline Second Order DSMC

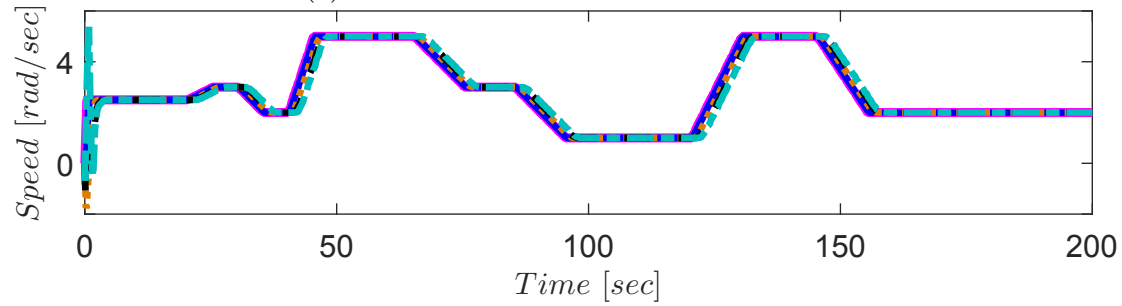

Figure 6.8: Comparison among the speed tracking results of the first and second order DSMCs for different sampling rates and quantization level of 16-bit: (a) SISO first order DSMC, (b) SISO second order DSMC, (c) MIMO second order DSMC. No model uncertainty is applied.

Figure 6.9 shows how the DSMC tracking performance could be affected by the quantization level of the ADC unit. As can be seen from Figure 6.9 a, the SISO first order DSMC is more sensitive to quantization level compared to SISO/MIMO second order 
(a) SISO Baseline First Order DSMC

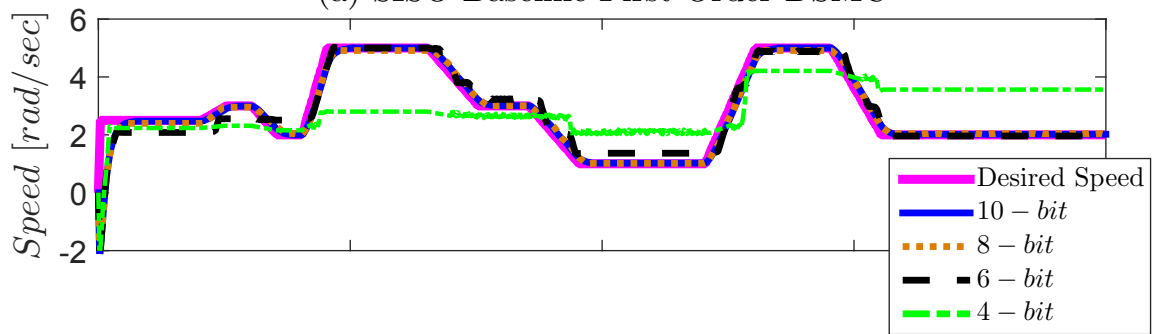

(b) SISO/MIMO Second Order DSMC

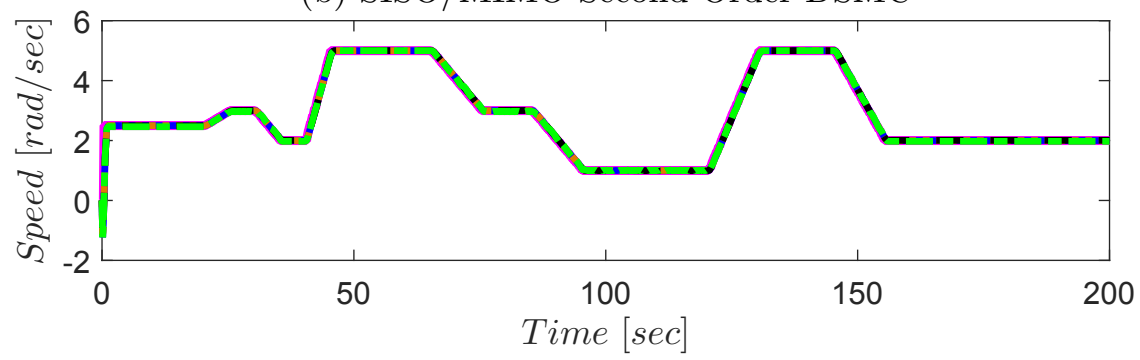

Figure 6.9: Comparison between the first and second order DSMCs in tracking the desired speed trajectory of the DC motor for different quantization levels. (a) SISO first order DSMC, (b) SISO/MIMO second order DSMC ( $T=200 \mathrm{~ms}$, no model uncertainty is applied).

DSMC. On the other side, any changes in the quantization level from 10-bit to 4-bit have no effect on both SISO and MIMO second order DSMCs tracking performances.

In order to show the effectiveness of incorporating the predicted ADC uncertainties in improving the DSMC against uncertainties, the second order DSMC is evaluated under extreme sampling and quantization levels which cause high level of imprecisions at the controller I/O. By looking into Figure 6.10, one can conclude the better tracking performance of the second order DSMC with predicted ADC uncertainties for both SISO and MIMO cases, compared to the two other controllers.

Another interesting feature of the MIMO second order DSMC in comparison with the SISO controller is its disturbance rejection characteristics. As shown in Eq. (5.56), 


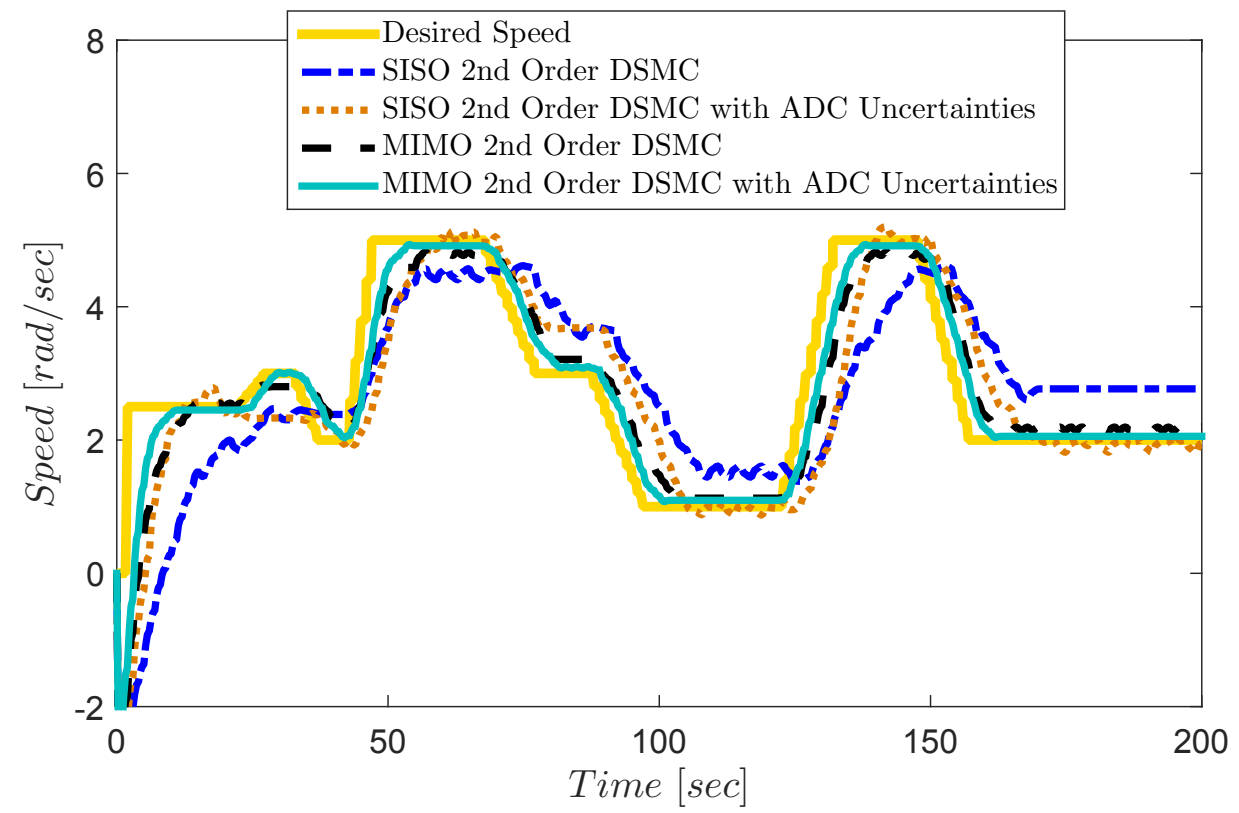

Figure 6.10: Comparison between the MIMO and SISO second order DSMCs, with and without predicted ADC uncertainties $\left(\mu_{u}\right)$, in tracking the desired speed trajectory under extreme ADC uncertainties $(T=1000 \mathrm{~ms}$, quantization level=4-bit, no model uncertainty is applied).

it is assumed that there is a constant torque $(\Gamma)$ on the shaft of the DC motor. If there will be any unknown disturbing torque on the motor shaft, the controller should reject the disturbance fast, to make sure the desired speed tracking is not affected. Figure 6.11 shows the disturbance rejection characteristics of the SISO and MIMO second order DSMCs, under different sampling and quantization levels. The disturbing torque, shown in Figure 6.11 $\mathrm{c}$, is defined with respect to the constant nominal torque on the DC motor shaft, and it generates up to $20 \%$ disturbing torque load. Figure 6.11-a shows that when the sampling rate is fast $(200 \mathrm{~ms})$, both SISO and MIMO second order DSMCs reject the disturbing torque effect very quickly. However, upon increasing the sampling rate from $200 \mathrm{~ms}$ to $800 \mathrm{~ms}$ (Figure 6.11-b), while the MIMO controller still shows accurate speed tracking and fast disturbance 
rejection results, the SISO controller fails to reject the disturbing torque impact and the trackino nerformance is affected sionificantlv.

(a)

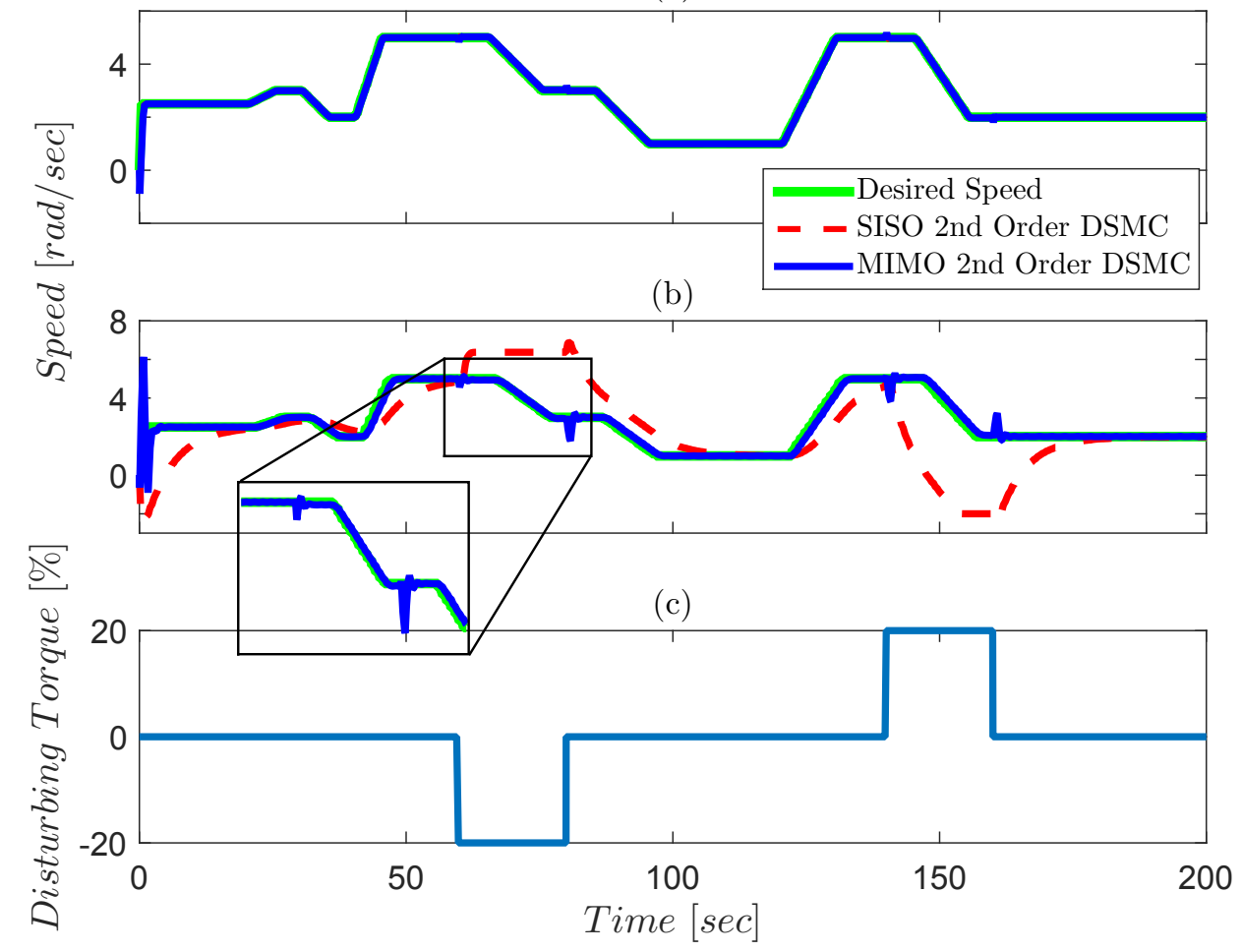

Figure 6.11: Disturbance rejection performance of the SISO and MIMO second order DSMCs for up to $20 \%$ sudden changes on the nominal external torque for (a) $T=200 \mathrm{~ms}$, quantization level=10-bit, (b) $T=800 \mathrm{~ms}$, quantization level=10-bit. Disturbing torque percentage is plotted in (c). No model uncertainty is applied.

Figure 6.12-a shows that at the extreme sampling rate $(1000 \mathrm{~ms})$ and quantization levels (4-bit), even the MIMO second order baseline DSMC fails to reject the disturbing torque (Figure 6.12-b). It is not a surprise that the disturbance rejection characteristics of the controller is weakened when the external uncertainties become larger. Figure 6.12-b shows that incorporation of the predicted ADC uncertainties (inclusion of the switching control input) helps to improve both tracking performance and 
disturbing torque rejection results, for both SISO and MIMO second order DSMCs compar

(a)

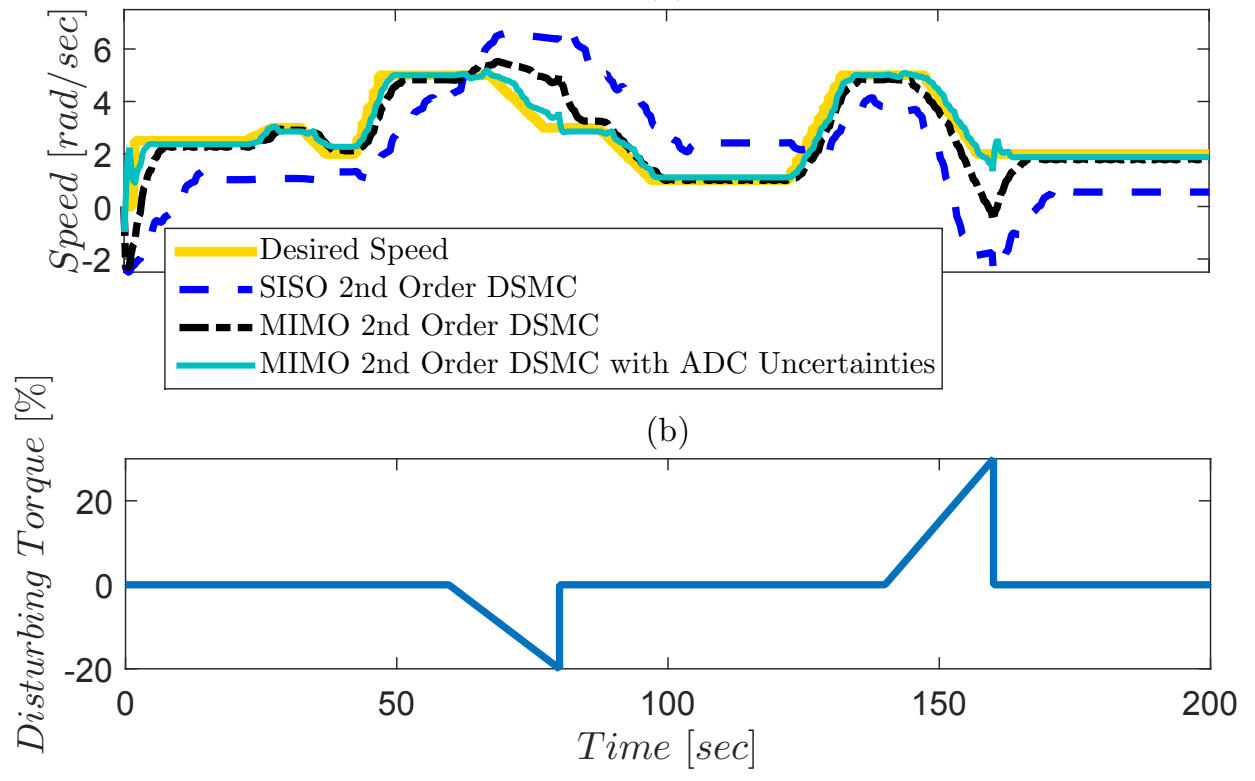

Figure 6.12: Disturbance rejection performance of the SISO and MIMO second order DSMCs, with and without predicted ADC uncertainties $\left(\mu_{u}\right)$, for up to $30 \%$ changes on the nominal external torque under extreme ADC uncertainties of $T=1000 \mathrm{~ms}$ and quantization level=4-bit, and (b) disturbing torque percentage. No model uncertainty is applied.

\subsubsection{Real-Time DC motor $2^{n d}$ order DSMC Verification}

The performance of the designed second order adaptive DSMC for the DC motor speed tracking problem is evaluated on an actual ECU within a PIL setup. The desired speed trajectory tracking performance of the controllers are studied in realtime under sampling time $=200 \mathrm{~ms}$ and quantization level=10 bit on the feedback and control signals, in the presence of multiplicative type of model uncertainties inside the model-based controller structure. The performance of the adaptive SISO first order 
DSMC, and adaptive MIMO second order DSMC with incorporated implementation uncertainties are shown in Figure 6.13 for tracking the desired speed profile. As can be observed, the adaptive second order MIMO DSMC with predicted ADC uncertainties is able to significantly improve the tracking performance by $60 \%$ compared to the first order adaptive SISO DSMC.

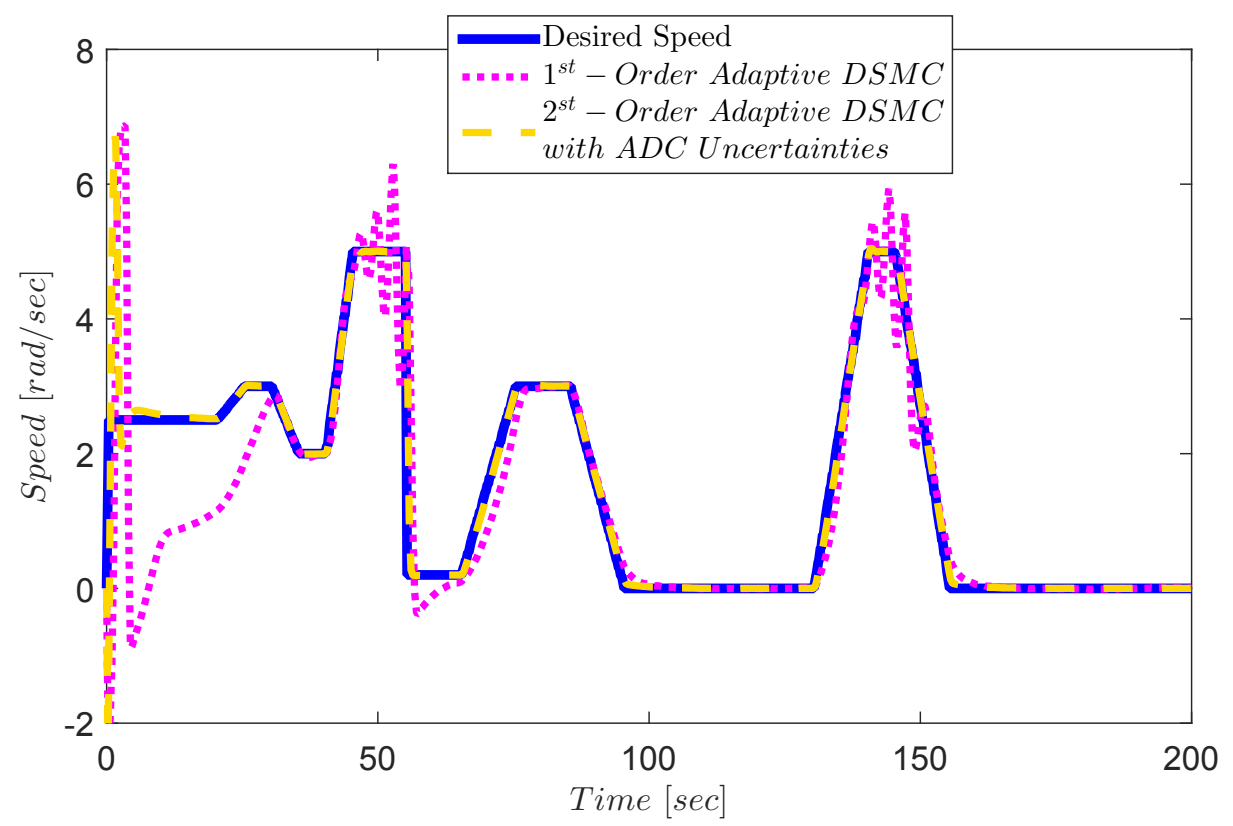

Figure 6.13: Real-time verification result of the DC motor speed control using the adaptive first order SISO DSMC and the modified adaptive second order MIMO DSMC with predicted ADC uncertainties. Test conditions: sampling time: $200 \mathrm{~ms}$, quantization level: 10-bit, 50\% model uncertainty is applied.

\subsection{Summary and Conclusion}

A new adaptive second order discrete sliding mode controller (DSMC) formulation for nonlinear uncertain systems was introduced in this paper. Based on the discrete Lyapunov stability theorem, an adaptation law was determined for removing generic unknown multiplicative uncertainty terms within the nonlinear difference equation 
of the plant's model. In order to ensure the controller robustness against external ADC imprecisions, a new switching control input was introduced, which contains the knowledge of $\mathrm{ADC}$ imprecisions via an online sampling and quantization uncertainties prediction and propagation mechanism. The proposed controller was examined for two case studies, including (i) DC motor speed control, (ii) spark ignition engine control problem to track desired air-fuel ratio, engine speed, and exhaust gas temperature trajectories. Comparing to the first order DSMC, the second order DSMC shows significantly better robustness against data sampling imprecisions, and can provide up to $80 \%$ improvement in terms of the tracking errors. The better performance of the second order DSMC can be traced in driving the higher order derivatives (difference functions) of the sliding variable to zero. In the presence of the model uncertainties, it was shown that the adaptation mechanism is able to remove the errors in the modeled dynamics quickly, and steer the dynamics towards their nominal values for both case studies. Increasing the sampling time raises the required time for the adaptation law to compensate for the uncertainties in the models. This required time was increased by two times, when the sampling time was increased from $10 \mathrm{~ms}$ to $40 \mathrm{~ms}$ in the engine tracking control problem, though the adaptation mechanism still could remove the model uncertainties in less than two seconds. 


\section{Chapter 7}

\section{Experimental Testing of the DSMC on an Electronic Throttle Body 1$]$}

\section{$7.1 \quad$ Introduction}

Air mass flow control into the intake manifold of internal combustion engines (ICEs) is an important part of vehicle powertrain control. Accurate air-to-fuel ratio $(A F R)$ and engine torque controls are achieved via air mass flow control [2]. Imprecise air mass flow control will result in undesired ICE performance, and directly affects vehicle drivability, fuel economy, and engine-out emissions. Air mass flow is modulated by an electromechanical throttle actuator, which is basically a DC motor which steers the throttle plate towards a desired position based on the demanded air mass flow from the ECU. Due to the nonlinear return spring with variable stiffness and significant

\footnotetext{
${ }^{1}$ This chapter has been published in the SAE International Journal of Commercial Vehicles 9 ] (doi:10.4271/2017-01-0598). Reprinted with permission by SAE @ 2017 SAE International as shown in Appendix D. Further distribution of this material is not permitted without prior permission from SAE.
} 
dry friction, the dynamics of the throttle is highly nonlinear. Because of this nonlinearity in the dynamics, classic control design techniques, such as PID controllers, are limited to provide accurate and fast throttle position tracking characteristics without extensive controller tuning.

Several linear and nonlinear control design techniques have been previously applied to the throttle position tracking problem. Studies in references [114, 115] presented a PID controller along with the nonlinear friction and limp-home compensators. Constrained time-optimal control problem for a piecewise affine (PWA) model of the throttle was presented in [116, 117]. The study in reference [118] demonstrated the application of adaptive pulse control (APC) to throttle angle control. A nonlinear control design was proposed utilizing the input-output feedback linearization approach in [119], and the controller was experimentally validated on a throttle body. A nonlinear feedforward and feedback controller based on flatness was designed in [120]; however, this approach was shown to be very sensitive to the parameter variations. Moreover, the application of linear parameter varying (LPV) methods for the electronic throttle control has been studied in the literature [121, 122, 123]. LPV methods allow for achieving the requirement of optimal control of the system performance over a certain operating range and improve the accuracy of the linearization of a nonlinear system [123]. A physics-based LPV model of the throttle was presented in [122], in which the highly nonlinear system is converted into an LPV system. Based on the 
LPV system, an $\mathrm{H}_{2}$ static output feedback control was designed to guarantee the system performance; however, it was observed that the system is not robust and small modeling error could make the system become unstable. This issue was addressed in [123], in which the mixed constrained $H_{2} / H_{\infty}$ LPV gain-scheduling control technique was investigated for the electronic throttle position control.

The capabilities of continuous-time sliding mode control (SMC) [124, 125, 126], and discrete-time sliding model control (DSMC) [127, 128, as robust and low-cost nonlinear control design techniques, have been shown in the literature for the throttle control problem. As it was discussed in details in the previous chapters, performance of previous model-based control techniques significantly deteriorates under imprecisions due to controller software implementation. In this chapter, the novel generic DSMC formulation from Chapter 4 including the predicted ADC imprecisions is employed to design and implement a position controller for an electromechanical throttle body.

The main contribution of this chapter is to (i) experimentally illustrate the importance of handling the ADC imprecisions in a model-based controller, and (ii) introduce a novel DSMC formulation for the throttle position control in order to improve the robustness characteristics against sampling and quantization imprecisions. 


\subsection{Throttle Experimental Setup}

Figure 7.1 shows the experimental setup developed in this study for open-loop and closed-loop throttle position regulations. The electronic throttle body is a ACDelco ${ }^{\circledR}$, Model 2173429, that uses a Throttle Position Sensor (TPS) for measuring angle of the throttle plate. The throttle housing width is $63.0 \mathrm{~mm}$. This throttle is the same as the throttle used on a GM 2-Liter Turbocharged I4 Ecotec Gen I LHU engine.

(a)
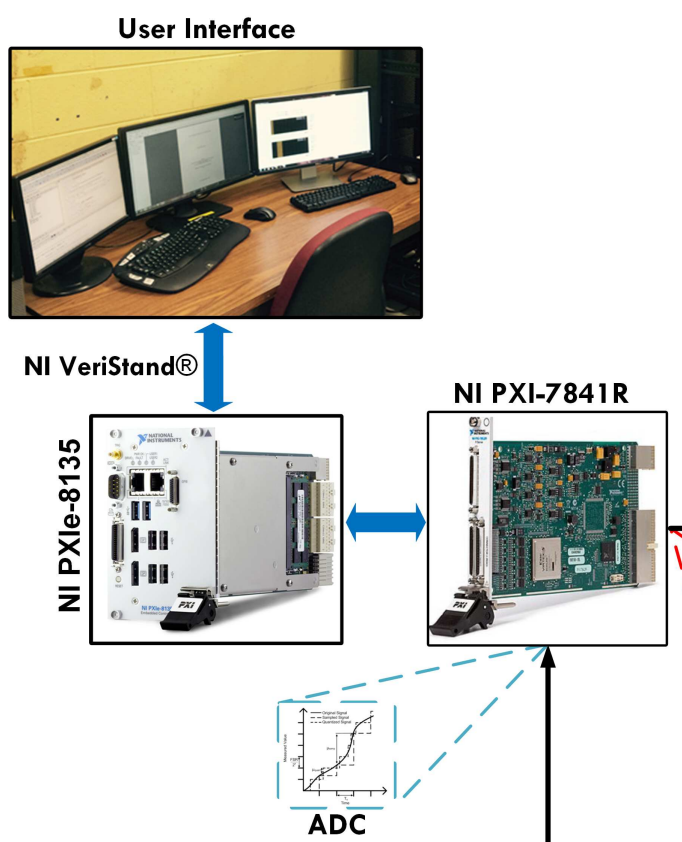

(b)

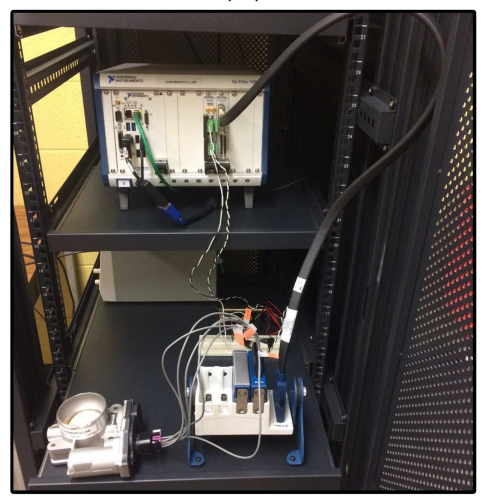

Control Commands

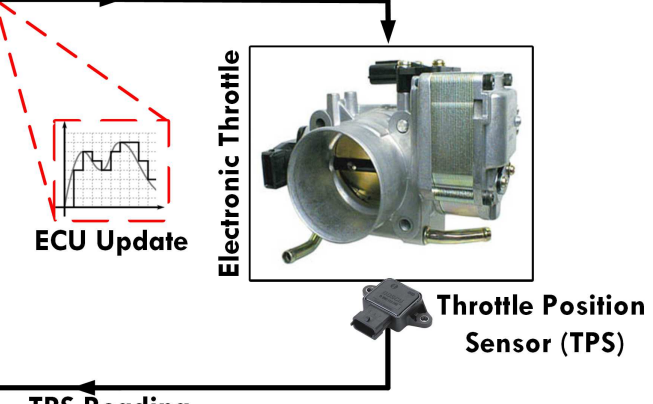

TPS Reading

Figure 7.1: Throttle experimental setup: (a) schematic, and (b) real system.

The ECU is a National Instrument (NI) PXI processor (NI PXIe-8135). PXI processor 
acquires and sends the signals from/to the throttle body via the NI PXI-7841R data acquisition card (DAQ), which is able to read/send the analog/digital signals with the quantization level of up to 16 bit. The throttle driver (full H-bridge) is a NI 9759 module which is connected to the PXI chassis (NI PXIe-1082) via a NI 9151 R-series expansion chassis. Real-time test configuration for NI PXI ECU is conducted using NI VeriStand ${ }^{\circledR}$ software on the user interface desktop computer. Access to the NI PXI possessor input/output (I/O) and interfaces (NI PXI-8135) is granted by VeriStand ${ }^{\circledR}$. Sampler and quantizer blocks are embedded on the throttle ECU I/Os to virtually emulate different sampling and quantization levels.

\subsection{Throttle Modelling and Parameter Identifica- tion}

The throttle has two main electrical and mechanical parts as shown in Figure 7.2 The electrical part is a DC motor armature which takes voltage $(U)$ as input and the output is torque on the output shaft. The generated torque by the DC motor is transferred to the shaft of the throttle via a gear set. The return spring on the throttle shaft brings the throttle back to the so-called "limp-home" region, if there would be any failure in the power supply [114]. To this end, the stiffness of the return spring in the limp-home region is set to be relatively high, compared to the normal position range of the throttle. The variable stiffness of the throttle return spring, and the limp-home region are demonstrated in the throttle torque response in Figure 7.3 . 
In addition to the limp-home region, there are two other regions in the spring with relatively high stiffness, which make the return spring dynamic more nonlinear. As can be seen from Figure 7.3 , these two regions are the areas near "Full Close" and "Full Open" positions. The high stiffness of the return spring at the full-close and fullopen positions represents the mechanical hardstops which limit the operation range of the throttle (i.e., $\varphi=0^{\circ}-90^{\circ}$ ).

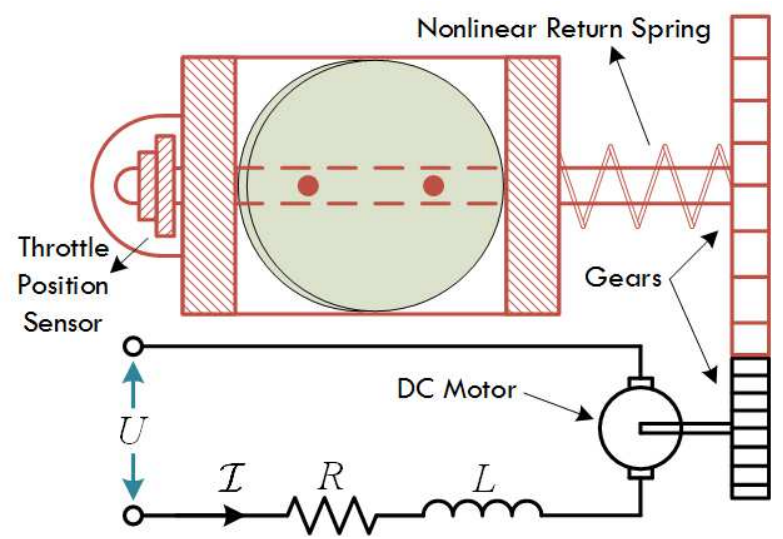

Figure 7.2: Schematic of the electronic throttle actuator.

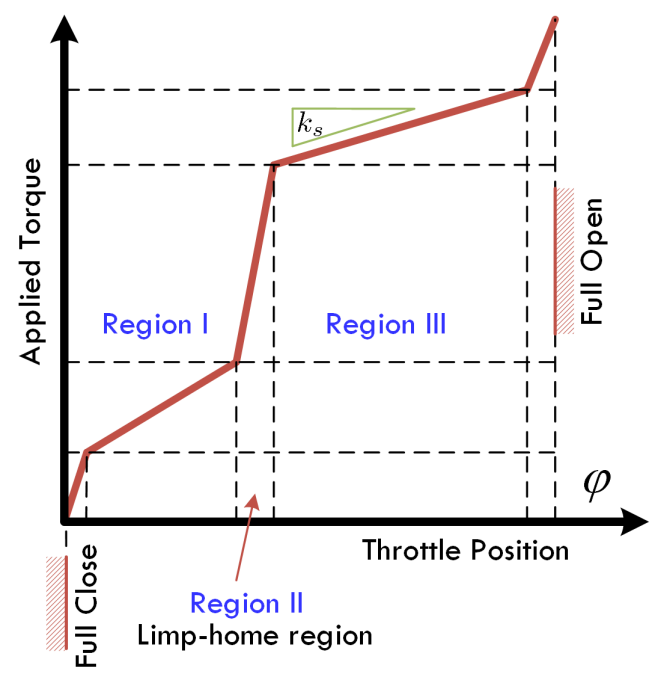

Figure 7.3: Effect of nonlinear return spring with variable stiffness $\left(k_{s}\right)$ on throttle torque-position response. 
Figures 7.4 and 7.5 show the open-loop experimental response of the throttle to the sinusoidal inputs with two different frequencies. The input, the duty cycle of the pulse width modulation $(\mathrm{PWM})$ signal, is based on $0.25 \sin (2 \pi \omega t)+0.5$ form, where $\omega$ is the input frequency. This input was applied to the throttle with $\omega=0.1 \mathrm{~Hz}$, and $0.5 \mathrm{~Hz}$. It can be observed that three important regions make the throttle dynamics highly nonlinear. These regions are limp-home region, and near full closing and opening regions. Inside limp-home region $\left(30-40^{\circ}\right)$, despite the increase in the applied input, the throttle position does not change. This behavior can be traced in the return spring which has a high stiffness in the limp-home region. Increasing the input frequency, due to the moment of inertia, decreases the operation range of the throttle. However, the dominant impact of the high stiff spring at limp-home, and full-open/close regions disappears. Ideally, at very high input frequency, those regions will disappear from the throttle dynamics completely, and similar to the applied input, the throttle behavior will be sinusoidal. Here, the objective is to capture the dominant parts of the throttle dynamics which cover the whole operation range of the throttle, and allow for the fast and accurate position tracking based on the ECU demand. To this end, mathematical models of different electrical/mechanical components of the throttle body will be discussed in the following.

The relation between current $(\mathcal{I})$ and the input voltage $(U)$ in the armature circuit of the DC motor is [129]:

$$
U=R \mathcal{I}+L \frac{d \mathcal{I}}{d t}+k_{e m f} \dot{\varphi}_{M}
$$



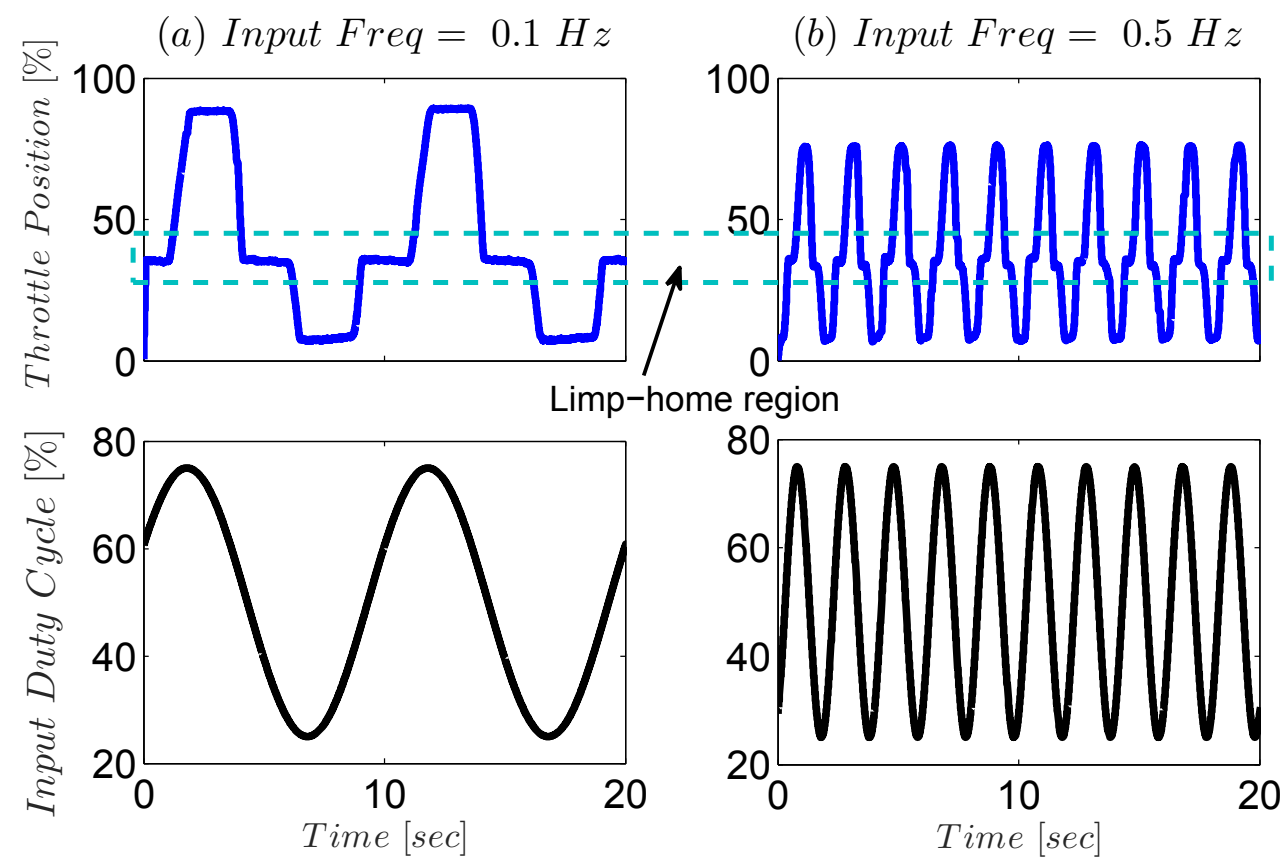

Figure 7.4: Open-loop experimental results of testing the throttle by applying $0.25 \sin (2 \pi \omega t)+0.5$ as the input at $\omega=$ (a) $0.1 \mathrm{~Hz}$ and (b) $0.5 \mathrm{~Hz}$.
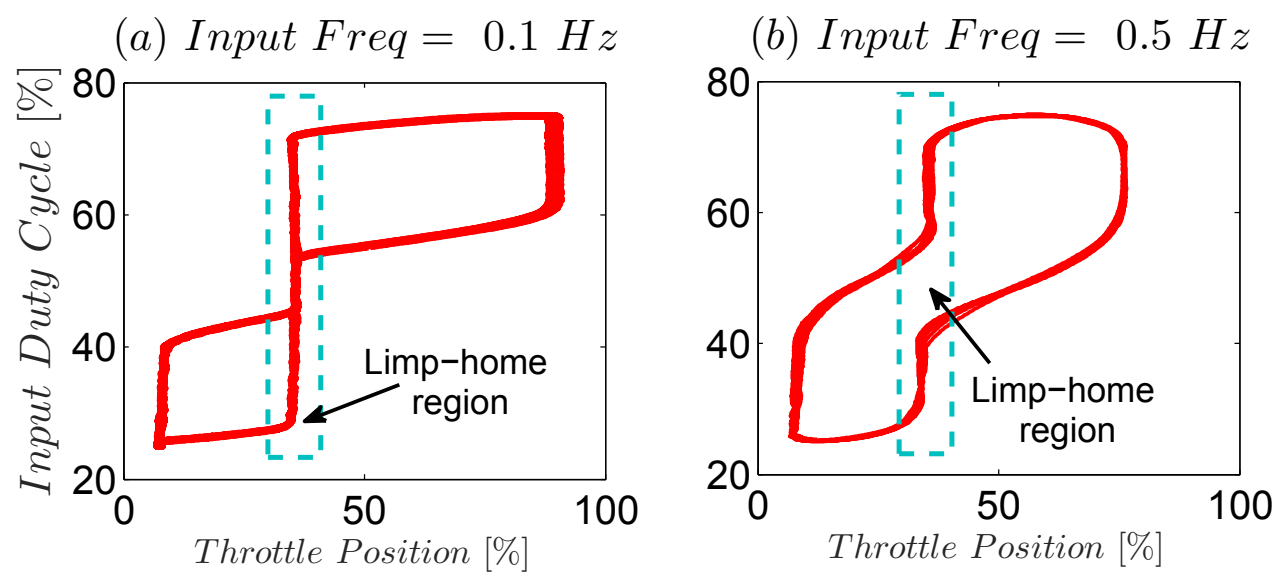

Figure 7.5: Effects of nonlinear return spring on the throttle position dynamic at different input frequencies. The experimental data are collected from the throttle by applying $0.25 \sin (2 \pi \omega t)+0.5$ as the input at (a) $\omega=0.1$ and (b) $\omega=0.5 \mathrm{~Hz}$.

where, $R$ is the electrical resistance, $L$ is the inductance, $\varphi_{M}$ is the DC motor shaft's position, and $k_{e m f}$ is the back electromotive force constant. Assuming a neglectable 
electrical dynamics $(L=0)$ [129, 130], the current $(\mathcal{I})$ can be calculated as follows:

$$
\mathcal{I}=\frac{1}{R}\left(U-k_{e m f} \dot{\varphi}_{M}\right)
$$

The electrical torque on the DC motor shaft $\left(\tau_{M}\right)$ is obtained according to the armature circuit current:

$$
\tau_{M}=k_{e m f} \mathcal{I}
$$

Next, $\tau_{M}$ is transferred to the throttle shaft via gears. The relation between DC motor shaft position $\left(\varphi_{M}\right)$ and the throttle position $(\varphi)$ is calculated by:

$$
\varphi_{M}=N \varphi
$$

where, $N$ is the total gear ratio from the DC motor shaft to the throttle's shaft. Similarly, the transferred torque from the DC motor to the throttle shaft $(\tau)$ is calculated by:

$$
\tau=N \eta \tau_{M}
$$

where, $\eta$ is the overall efficiency of the mechanical system. By substituting Eq. (7.1)(7.4) into Eq. (7.5), $\tau$ can be expressed with respect to the input voltage:

$$
\tau=\frac{N k_{e m f} \eta}{R} U-\frac{N^{2} k_{e m f}^{2} \eta}{R} \dot{\varphi}
$$

The following equation describes the system dynamics on the mechanical side:

$$
J \ddot{\varphi}=\tau-\tau_{v}-\tau_{s}-\tau_{f}
$$

where, $\tau_{v}$ is the mechanical viscous torque; $\tau_{s}$ is the torque from the nonlinear return 
spring; $\tau_{f}$ is the dry friction torque; $J$ is the throttle moment of inertia. $\tau_{v}$ is calculated as follows:

$$
\tau_{v}=b_{m e c h} \dot{\varphi}
$$

where, $b_{\text {mech }}$ is the mechanical viscous damping coefficient. $\tau_{s}$, the generated torque by the nonlinear return spring, is determined by:

$$
\tau_{s}=k_{s}(\varphi) \varphi
$$

where, $k_{s}(\varphi)$ is not constant and is a function of the throttle position (Figure 7.3). Finally, the torque loss due to dry friction $\left(\tau_{f}\right)$ is calculated using the following equation [124, 131]:

$$
\tau_{f}=\mu \xi \operatorname{sgn}(\dot{\varphi})
$$

where, $\mu$ and $\xi$ are the friction coefficient and vertical force, respectively. By substituting Eq. (7.6), and (7.8)-7.10) into Eq. (7.7), the mechanical dynamics is presented by:

$$
\begin{gathered}
J \ddot{\varphi}=\frac{N k_{e m f} \eta}{R} U-\frac{N^{2} k_{e m f}^{2} \eta}{R} \dot{\varphi} \\
-b_{m e c h} \dot{\varphi}-k_{s}(\varphi) \varphi-\mu \xi \operatorname{sgn}(\dot{\varphi})
\end{gathered}
$$

Eq. 7.11 is normalized with respect to control input $U$ by multiplying $\frac{R}{N k_{e m f} \eta}$ to the both sides of the equation. This yields [130]:

$$
J^{*} \ddot{\varphi}=U-b^{*} \dot{\varphi}-K^{*}(\varphi) \varphi-\mu^{*} \operatorname{sgn}(\dot{\varphi})
$$


Normalized parameters in Eq. (7.12) are noted by superscript '*' and are calculated by:

$$
\begin{aligned}
J^{*} & =\frac{R}{N k_{e m f} \eta} J \\
b^{*} & =\frac{R}{N k_{e m f} \eta}\left(\frac{N^{2} k_{e m f}^{2} \eta}{R}+b_{\text {mech }}\right) \\
K^{*} & =\frac{R}{N k_{e m f} \eta} k_{s}(\varphi) \\
\mu^{*} & =\frac{R}{N k_{e m f} \eta} \mu \xi
\end{aligned}
$$

The parameters of the throttle dynamics in Eq. (7.13) are estimated based on the open-loop experimental data which are collected by applying different control inputs to make sure the dominant parts of the dynamics are captured. To take into account the nonlinear behavior of the spring and keep the model as simple as possible, a switching model with different sets of parameters is identified. The model switches to the corresponding set of the parameters based on the throttle position. Three sets of parameters are listed in Table 7.1. The first set covers full-close to limp-home region (Region I in Figure 7.3). Second set is dedicated to the limp-home region (Region II in Figure 7.3), while the third set covers the region between limp-home area to the full-open position (Region III in Figure 7.3). A nonlinear least square method is used to find each set of parameters using MATLAB ${ }^{\circledR}$ System Identification Toolbox. Figure 7.6 shows the results of validating the identified model against the open-loop experimental data. The verification results show that the model is able to capture the dominant part of the throttle dynamics with an overall mean error of $1.5^{\circ}$. 
Table 7.1

Identified parameters of the throttle model in Eq. (7.13), according to the operating regions in Figure 7.3 .

\begin{tabular}{c|c|c|c} 
Parameter & $\begin{array}{c}\text { Set 1 } \\
(\text { Region I })\end{array}$ & $\begin{array}{c}\text { Set 2 } \\
(\text { Region II })\end{array}$ & $\begin{array}{c}\text { Set 3 } \\
(\text { Region III })\end{array}$ \\
\hline \hline$J^{*}$ & $1.89 \times 10^{-6}$ & $2.96 \times 10^{-7}$ & $1.62 \times 10^{-6}$ \\
\hline$b^{*}$ & $5.48 \times 10^{-3}$ & $6.33 \times 10^{-3}$ & $5.75 \times 10^{-3}$ \\
\hline$K^{*}$ & 0.63 & 0.95 & 0.79 \\
\hline$\mu^{*}$ & $3.58 \times 10^{-4}$ & $3.21 \times 10^{-3}$ & $3.50 \times 10^{-4}$ \\
\hline \hline
\end{tabular}

(a)
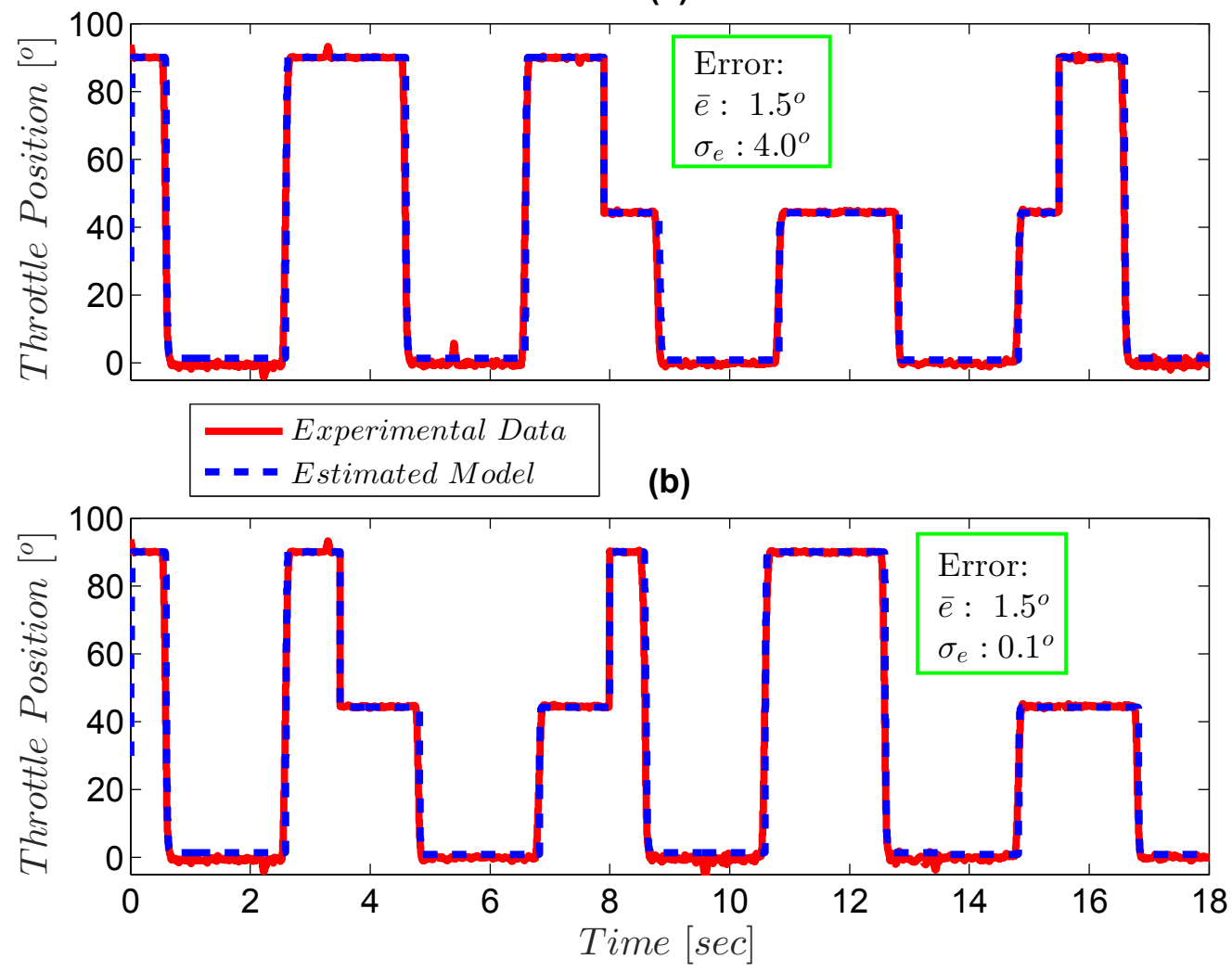

Figure 7.6: Results of throttle model verification against two sets of experimental data ( $\bar{e}$ and $\sigma$ are the mean and standard deviation errors, respectively). 


\subsection{DSMC design for Throttle Position Control}

The state space representation of the throttle dynamics can be expressed using the following second order system by assuming $x_{1}=\varphi$ and $x_{2}=\dot{\varphi}$ as the system states:

$$
\begin{aligned}
& \dot{x}_{1}(t)=x_{2}(t) \\
& \dot{x}_{2}(t)=\frac{1}{J^{*}}\left(U(t)-b^{*} x_{2}(t)-K^{*} x_{1}(t)-\mu^{*} \operatorname{sgn}\left(x_{2}(t)\right)\right)
\end{aligned}
$$

The discrete difference equations of the model can be obtained using the first order Euler approximation [89]:

$$
\begin{aligned}
x_{1}(i+1) & =x_{1}(i)+T x_{2}(i) \\
x_{2}(i+1) & =x_{2}(i) \\
+ & \frac{T}{J^{*}}\left(U(i)-b^{*} x_{2}(i)-K^{*} x_{1}(i)-\mu^{*} \operatorname{sgn}\left(x_{2}(i)\right)\right)
\end{aligned}
$$

The first sliding variable $\left(s_{1}\right)$ is defined to be the error in tracing the desired throttle position $\left(s_{1}(i)=\varphi(i)-\varphi_{d}(i)\right)$. As can be seen from Eq. (7.15), there is no direct control input on throttle position. On the other side, the voltage $(U)$ is the input for the second state equation $\left(x_{2}\right)$. In order to link the first and second state variables, and indirectly apply the voltage to the throttle position, a synthetic control input is defined. The synthetic control input is first calculated from the first sliding reaching law. Next, it will be used as the desired value for the second sliding variable. Applying the sliding reaching law (Eq. (5.36) ) to the first sliding variable yields: 


$$
\begin{gathered}
s_{1}(i+1)=x_{1}(i+1)-x_{1 d}(i+1)=\rho_{1} s_{1}(i) \rightarrow \\
x_{1}(i)+T x_{2}(i)-x_{1 d}(i+1)=\rho_{1} s_{1}(i) \rightarrow \\
x_{2 d}(i)=\frac{1}{T}\left(\rho_{1} s_{1}(i)-x_{1}(i)+x_{1 d}(i+1)\right)
\end{gathered}
$$

where, $x_{2 d}$ is the calculated synthetic control input which will be used to define the second sliding variable $\left(s_{2}\right)$. It is assumed that $s_{2}(i)=x_{2}(i)-x_{2 d}(i)$, where $x_{2}$ is the throttle speed $(\dot{\varphi})$, and $x_{2 d}$ is calculated from Eq. (7.16). Since the only measured signal is the throttle position, the derivative of the throttle position is estimated using a third order Euler approximation:

$$
x_{2}(i)=\frac{-\frac{1}{3} \varphi(i-3)+\frac{3}{2} \varphi(i-2)-3 \varphi(i-1)+\frac{11}{6} \varphi(i)}{T}
$$

The main control input $(U)$ is calculated by applying the sliding reaching law to $s_{2}$ :

$$
\begin{gathered}
s_{2}(i+1)=x_{2}(i+1)-x_{2 d}(i+1)=\rho_{2} s_{2}(i) \rightarrow \\
x_{2}(i)+\frac{T}{J^{*}}\left(U(i)-b^{*} x_{2}(i)-K^{*} x_{1}(i)-\mu^{*} \operatorname{sgn}\left(x_{2}(i)\right)\right) \\
-x_{2 d}(i+1)=\rho_{2} s_{2}(i)
\end{gathered}
$$

which yields:

$$
\begin{gathered}
U(i)=\frac{J^{*}}{T}\left(\rho_{2} s_{2}(i)-x_{2}(i)+x_{2 d}(i+1)\right) \\
\left(b^{*} x_{2}(i)+K^{*} x_{1}(i)+\mu^{*} \operatorname{sgn}\left(x_{2}(i)\right)\right)
\end{gathered}
$$

$x_{2 d}(i+1)$ in Eq. (7.19) is obtained by expanding Eq. (7.16): 


$$
\begin{gathered}
x_{2 d}(i+1)=\frac{1}{T}\left(\rho_{1}\left(T x_{2}(i)+x_{1}(i)-x_{1 d}(i+1)\right)\right. \\
\left.-T x_{2}(i)+x_{1}(i)+x_{1 d}(i+2)\right)
\end{gathered}
$$

By comparing Eq. (7.19) and (7.20), it can be seen that the calculated control input at $i^{\text {th }}$ time step is a function of $x_{1 d}$ at $i, i+1, i+2$ time steps. This means that the control input at $i^{\text {th }}$ time step considers the desired trajectories with a 2-step horizon. The inherent receding characteristics of the designed DSMC leads to better understanding of the desired throttle position profile, and consequently better tracking performances. Eq. (7.19) determines the value of the control input of the baseline DSMC. In the next step, the baseline control law is modified against ADC imprecision by incorporating the propagated sampling and quantization uncertainties on the control input into the DSMC formulation. To this end, first it is required to estimate the ADC uncertainties on control signal $\left(\mu_{U}\right)$.

The proposed technique in Figure 4.2 is employed for online ADC uncertainty prediction and propagation in real-time. Upon successful implementation of the uncertainty prediction algorithm, the estimated $\mu_{U}$ is incorporated into the baseline DSMC control input according to Eq. 4.22 as follows:

$$
\begin{gathered}
U_{\text {mod }}(i)=\frac{J^{*}}{T}\left(\rho_{2} s_{2}(i)-x_{2}(i)+x_{2 d}(i+1)\right) \\
\left(b^{*} x_{2}(i)+K^{*} x_{1}(i)+\mu^{*} \operatorname{sgn}\left(x_{2}(i)\right)\right)-\mu_{U}(i) s_{2}(i)
\end{gathered}
$$


Figure 7.7 shows the simulation results of testing the baseline DSMC for tracking the desired throttle position profile under $1 \mathrm{~ms}, 2 \mathrm{~ms}$, and $5 \mathrm{~ms}$ sampling times, and 16 bit quantization on the signals at the controller I/O. The significant effect of the sampling time on the controller performance can be observed from Figure 7.7, when at $T=1 \mathrm{~ms}$, the controller tracks the desired position accurately. However, once the sampling time increases, the baseline DSMC tracking performance degrades from its ideal case.

(a)

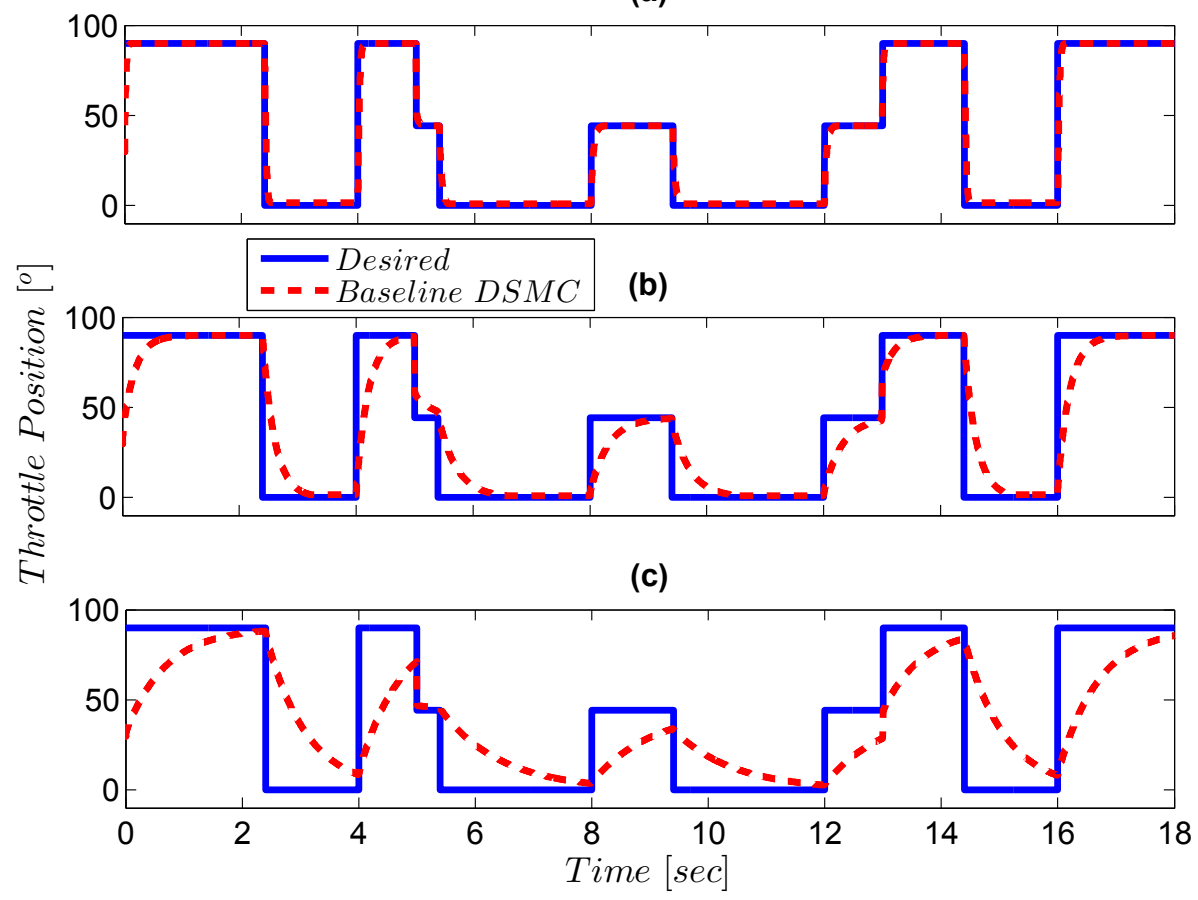

Figure 7.7: Simulation results of the baseline DSMC for tracking the desired throttle position under quantization level of $16 \mathrm{bit}$, and (a) $T=1 \mathrm{~ms}$, (b) $T=2 \mathrm{~ms}$, and (c) $T=5 \mathrm{~ms}$.

The results of the ADC uncertainty prediction on the throttle position measured signal are shown in Figure 7.8. The high accuracy of the uncertainty prediction mechanism can be concluded from the mean error of the introduced sampling and 


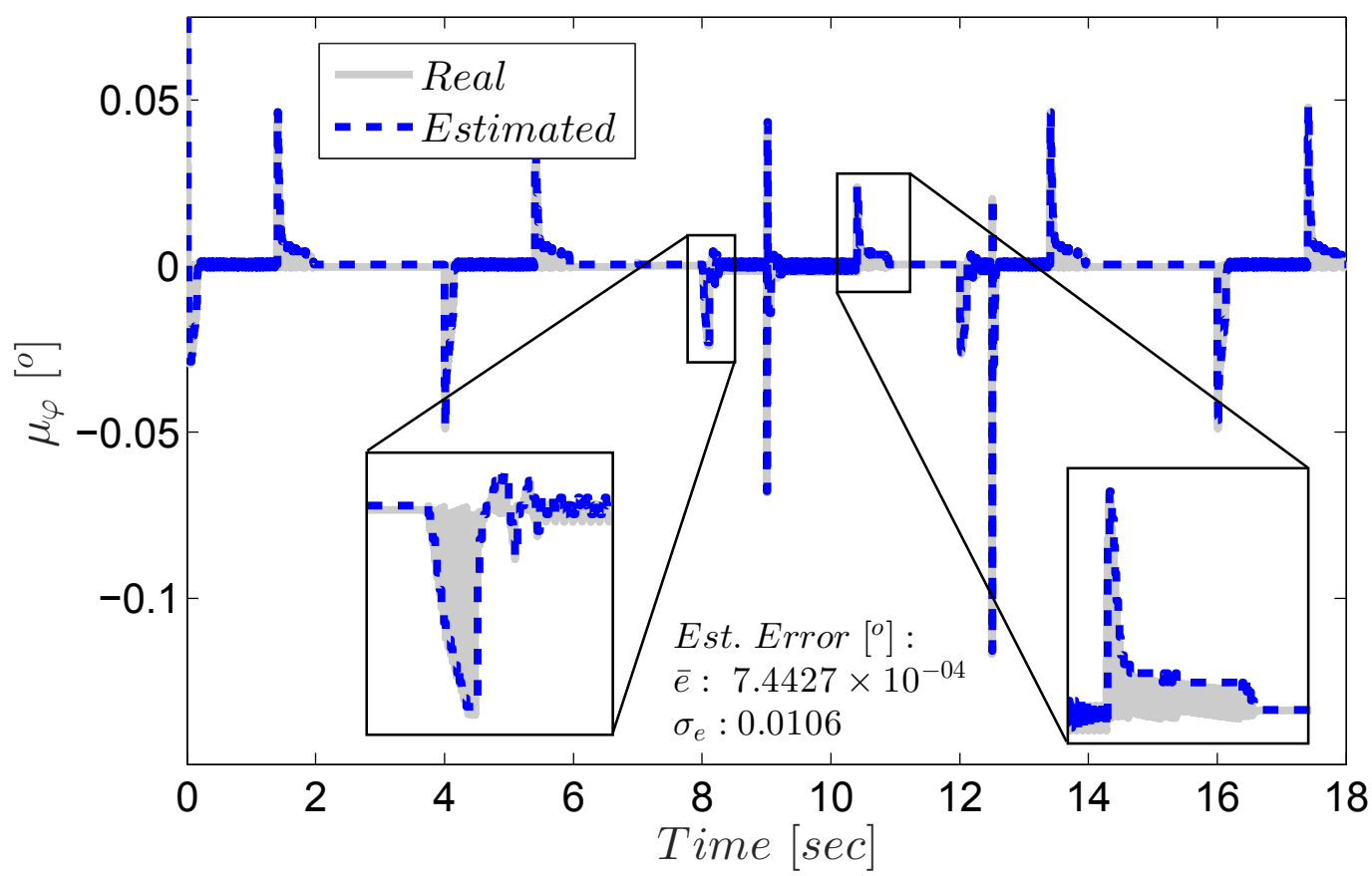

Figure 7.8: Comparison of actual and predicted uncertainties due to sampling and quantization on measured throttle position $(5 \mathrm{~ms}$ sampling time and 16-bit quantization level. $\bar{e}$ is the mean error, and $\sigma$ is the standard deviation error).

quantization imprecisions estimation. Next, using the uncertainty propagation technique, shown in Figure 4.2, the ADC uncertainties are incorporated into the DSMC. Figure 7.9 illustrates the effectiveness of the designed robust DSMC in enhancing the baseline controller robustness against sampling and quantization imprecisions. While the baseline controller fails to offer a precise and fast tracking performance under $5 \mathrm{~ms}$ of sampling time and quantization level of 16 bit, the robust DSMC with predicted ADC imprecisions shows significantly better results under the same conditions.

The impact of quantization level on the baseline DSMC is shown in Figure 7.10 for $T=2 \mathrm{~ms}$. It can be observed that the introduced uncertainty due to quantization will increase by lowering the quantization level. When the quantization level is 6 bit, 


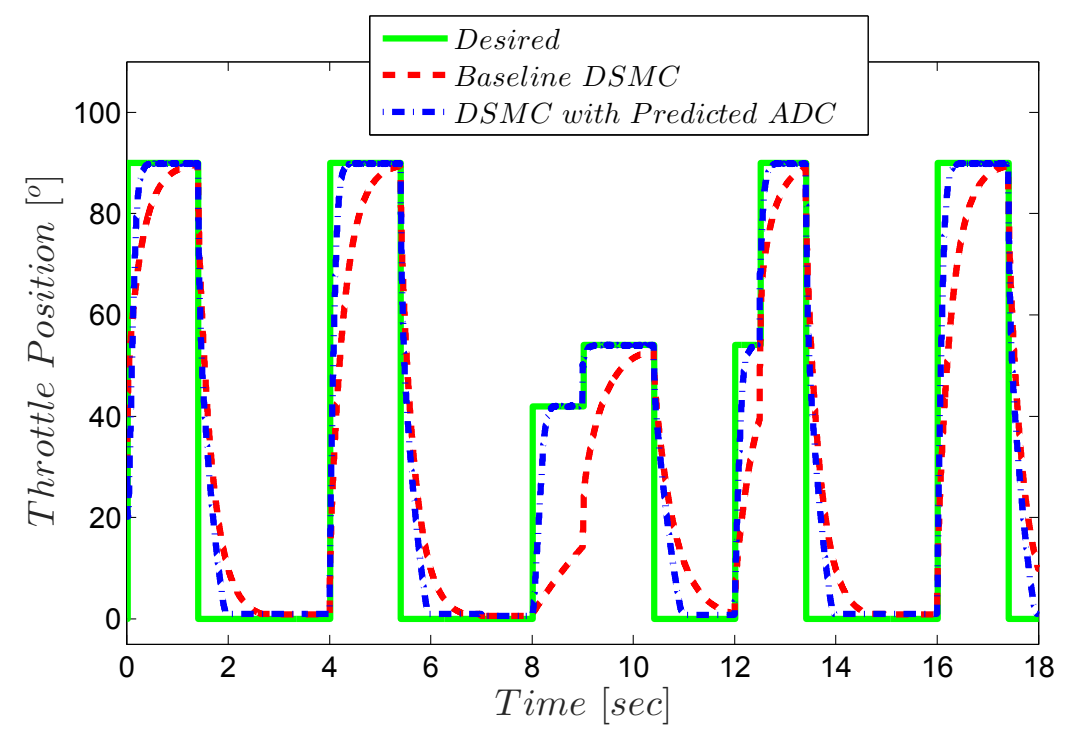

Figure 7.9: Simulation results of the baseline DSMC and robust DSMC with predicted sampling uncertainties for tracking the desired throttle position $(T=5 \mathrm{~ms}$, and quantization level=16 bit).
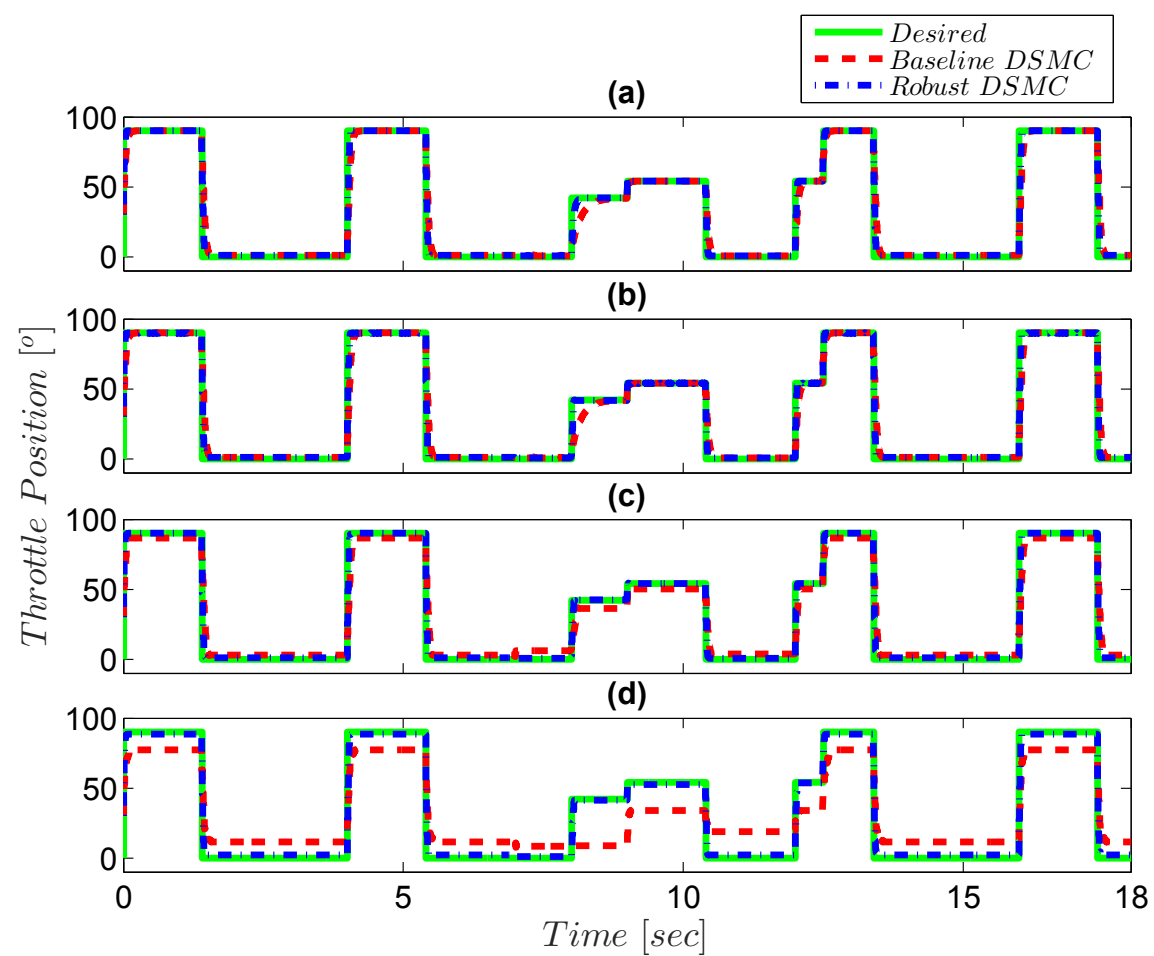

Figure 7.10: Simulation results of the baseline DSMC and robust DSMC with predicted ADC uncertainties for tracking the desired throttle position under $2 \mathrm{~ms}$ of sampling time and (a) no quantization, (b) 10 bit, (c) 8 bit, and (d) 6 bit. 
the baseline DSMC fails to track the desired positions and the tracking error rises by $80 \%$ compared to the ideal case, in which there is no quantization. On the other side, by incorporating the predicted ADC uncertainties into the baseline DSMC structure, Figure 7.10 verifies that the robust DSMC removes the ADC imprecisions effects and the controller performs similar to the ideal controller, with minimum tracking errors.

Figure 7.11 shows the experimental results of testing the baseline DSMC for different desired position trajectories, while the signals to the controller I/O are sampled every $1 \mathrm{~ms}$, and the quantization level is 16 bit. These experimental results verify the simulation test results in Figure 7.7. In addition, when the experiments were conducted under sampling time of $5 \mathrm{~ms}$, in Figure 7.12 , the experimental observations are similar to those in Figure 7.7. The experimental results show that performance of the baseline DSMC is affected significantly by the sampling imprecisions.

Finally, the proposed robust DSMC with predicted ADC uncertainties was experimentally tested on the throttle under different sampling and quantization conditions to verify the effectiveness of the proposed approach in enhancing the controller performance against ADC imprecisions. Figure 7.13 shows the experimental test results under sampling time of $5 \mathrm{~ms}$ and quantization level of $10 \mathrm{bit}$. It can be seen that the baseline DSMC $\left(\mu_{U}=0\right)$ tracks the desired trajectories slowly and the tracking errors are high $\left(\bar{e}=6.8^{\circ}\right)$. Once the robust DSMC is activated, the controller response becomes faster and the tracking errors decreases by $70 \%$ on average $\left(\bar{e}=0.1^{\circ}\right)$. 
(a)

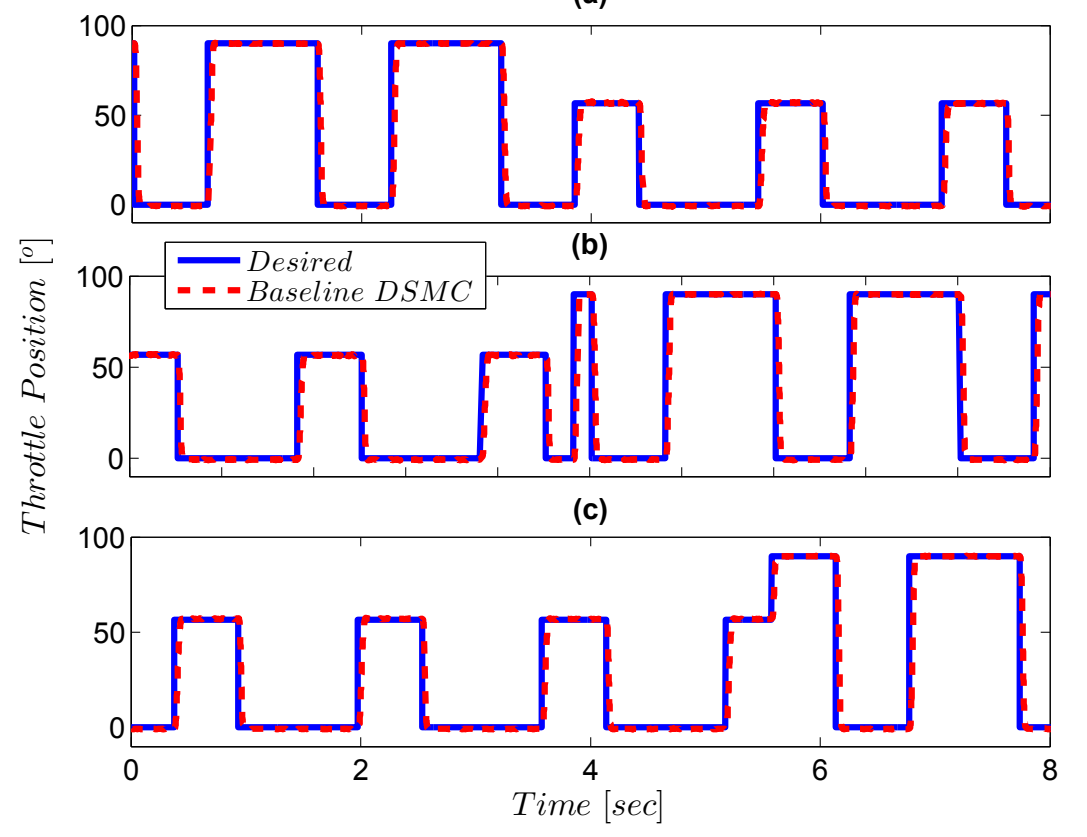

Figure 7.11: Experimental testing results of the baseline DSMC for tracking the desired throttle position at $T=1 \mathrm{~ms}$, and quantization level=16 bit for different desired position trajectories.

(a)
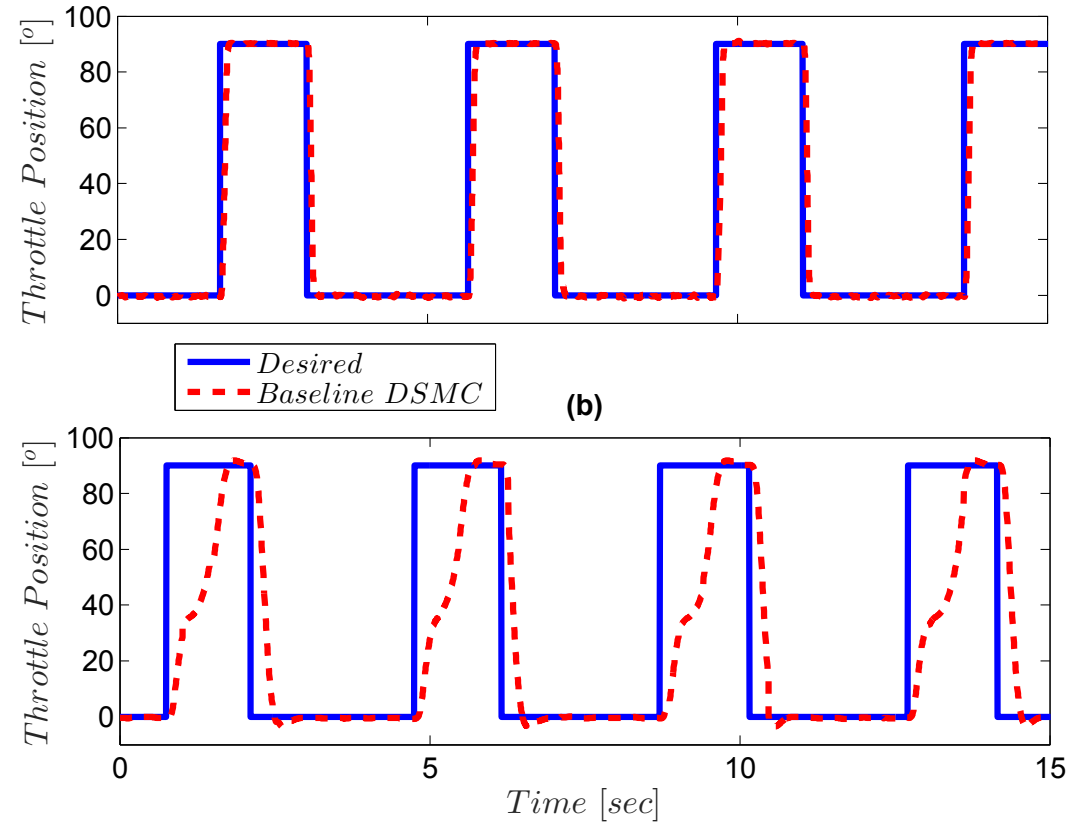

Figure 7.12: Experimental testing results of the baseline DSMC for tracking the desired throttle position: (a) $T=1 \mathrm{~ms}$, (b) $T=5 \mathrm{~ms}$ (quantization level $=16$ bit in both cases). 


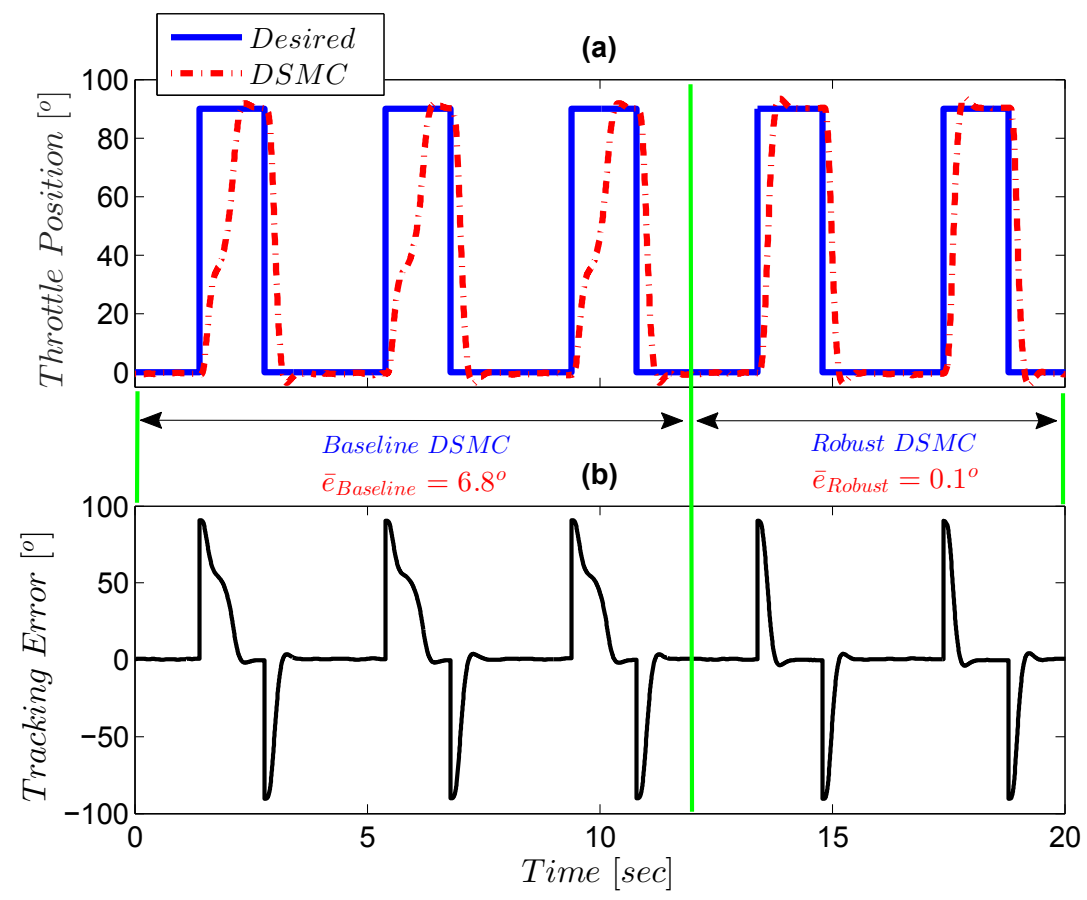

Figure 7.13: Experimental testing results of the baseline and robust DSMC for tracking the desired throttle position ( $T=5 \mathrm{~ms}, n=10 \mathrm{bit})$.

Figure 7.14 shows the experimental test results under very low quantization level and large sampling time, where the signals are digitized at the quantization level of 6 bit. Due to the substantial ADC uncertainty, the baseline DSMC cannot even move the throttle. However during the period that the robust DSMC is active, the throttle recovers from the dead condition and tries to follow the desired position at its best, under an extreme ADC condition.

\subsection{Second Order DSMC for Throttle Position Control}

In this section, the effectiveness of the second order DSMC in improving the controller robustness against ADC imprecisions is investigated. The control input of the second 


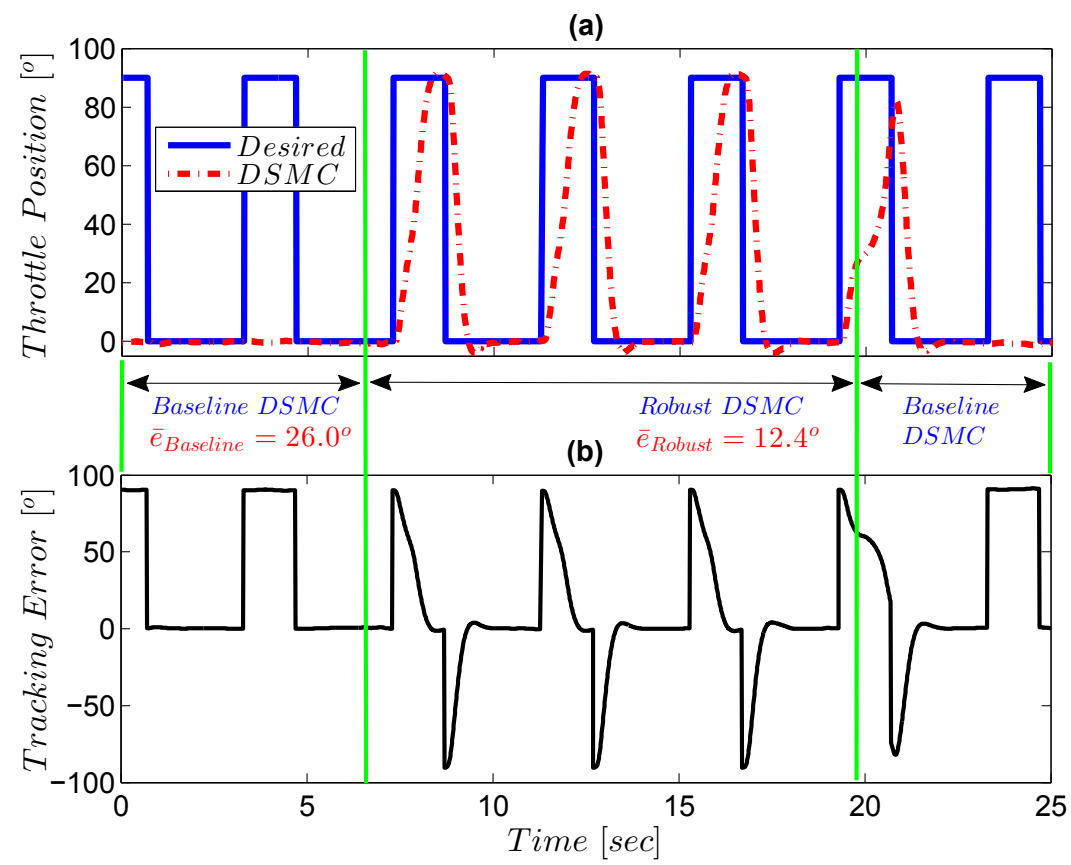

Figure 7.14: Experimental testing results of the baseline and robust DSMC for tracking the desired throttle position ( $T=10 \mathrm{~ms}, n=6 \mathrm{bit})$.

order DSMC, based on the definition of the first order sliding variables $\left(s_{1}, s_{2}\right)$ in Eq. (7.16) and (7.18), is found by using Eq. 6.17):

$$
\begin{gathered}
U(i)=\frac{J^{*}}{T}\left(-\beta_{2} s_{2}(i)-x_{2}(i)+x_{2 d}(i+1)\right) \\
\left(b^{*} x_{2}(i)+K^{*} x_{1}(i)+\mu^{*} \operatorname{sgn}\left(x_{2}(i)\right)\right)
\end{gathered}
$$

in which, $x_{2 d}$ is calculated as follows:

$$
\begin{aligned}
x_{2 d}(i+1) & =\frac{1}{T}\left(-\beta_{1}\left(T x_{2}(i)+x_{1}(i)-x_{1 d}(i+1)\right)\right. \\
& \left.-T x_{2}(i)+x_{1}(i)+x_{1 d}(i+2)\right)
\end{aligned}
$$

Figure 7.15 shows the comparison results of testing the first and second order baseline DSMCs under different sampling times. Table 7.2 summarizes the comparison results between the first and second order DSMCs in terms of the mean $(\bar{e})$ and standard 
deviation $\left(\sigma_{e}\right)$ of the tracking errors. Similar to the DC motor and engine case studies in Chapter 6, Figure 7.15 and Table 7.2 show the higher robustness characteristics of the second order DSMC against implementation imprecisions for the throttle body case study. The results from Table 7.2 illustrates that the baseline second order DSMC improves the throttle position tracking performance by $60-90 \%$, in comparison with the first order DSMC. Figure 7.15 shows that once the sampling time is increased from $1 \mathrm{~ms}$ to $10 \mathrm{~ms}$, the first order DSMC fails to track the desired throttle position. On the other side, despite the increase in the sampling time, the second order DSMC provides fast and accurate tracking performance in all cases.

Table 7.2

Comparison results between the first and second order DSMCs for tracking the desired throttle position in terms of the mean $(\bar{e})$ and standard deviation $\left(\sigma_{e}\right)$ of the tracking errors (Values inside the parentheses show the resulting improvement from the second order DSMC compared to the baseline first order DSMC).

\begin{tabular}{|c|c|c|c|c|}
\hline \multirow{4}{*}{$\begin{array}{c}\text { Sampling } \\
\text { Time } \\
T \\
\end{array}$} & \multicolumn{2}{|c|}{$\bar{e}\left[^{o}\right]$} & \multicolumn{2}{|c|}{$\sigma_{e}[o]$} \\
\hline & First Order & Second Order & First Order & Second Order \\
\hline & DSMC & DSMC & DSMC & DSMC \\
\hline & (Ref) & & (Ref) & \\
\hline $1 \mathrm{~ms}$ & 0.8 & 0.3 & 5.8 & 3.5 \\
\hline & & $(-62.5 \%)$ & & $(-39.7 \%)$ \\
\hline $2 m s$ & 3.3 & 0.4 & 10.1 & 3.9 \\
\hline & & $(-87.9 \%)$ & & $(-61.4 \%)$ \\
\hline $5 \mathrm{~ms}$ & 14.2 & 0.8 & 11.5 & 6.1 \\
\hline & & $(-94.3 \%)$ & & $(-17.3 \%)$ \\
\hline $10 \mathrm{~ms}$ & 32.7 & 2.6 & 12.7 & 10.5 \\
\hline & & $(-92.0 \%)$ & & $(-17.3 \%)$ \\
\hline
\end{tabular}


(a)

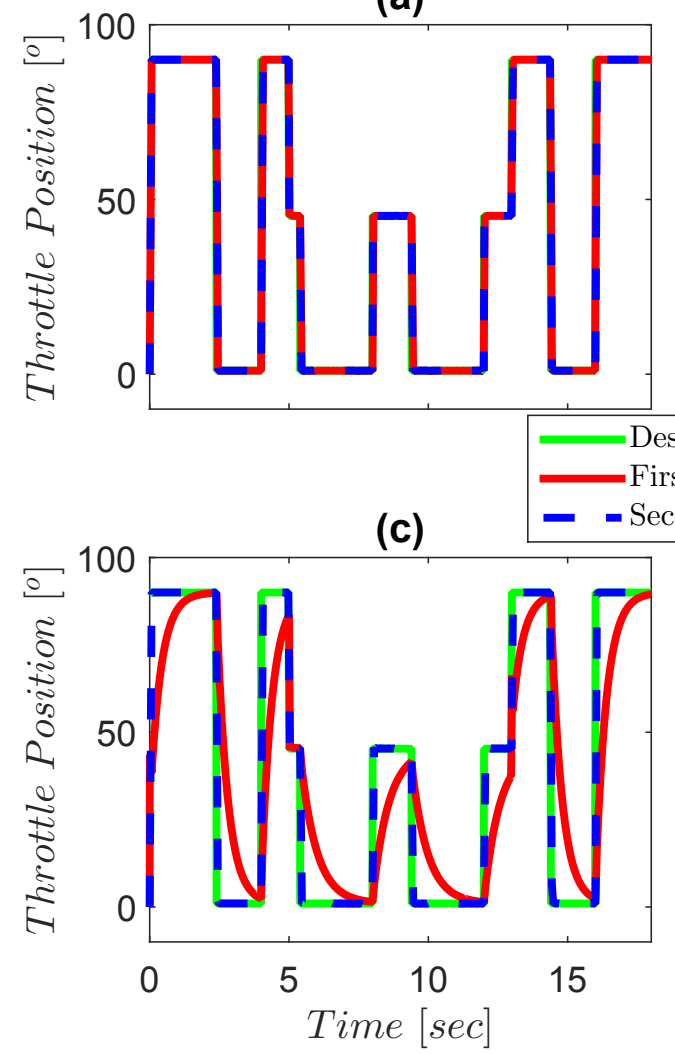

(b)

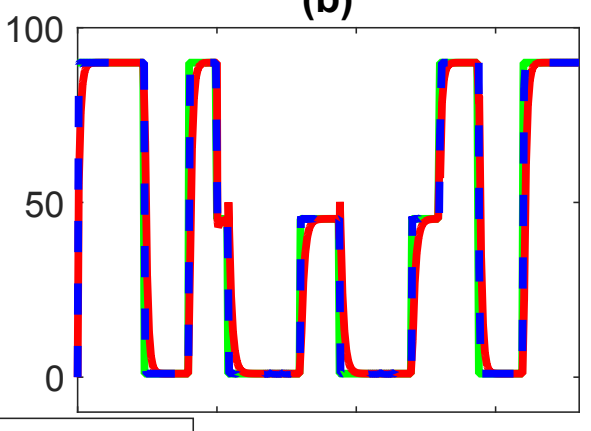

(d)

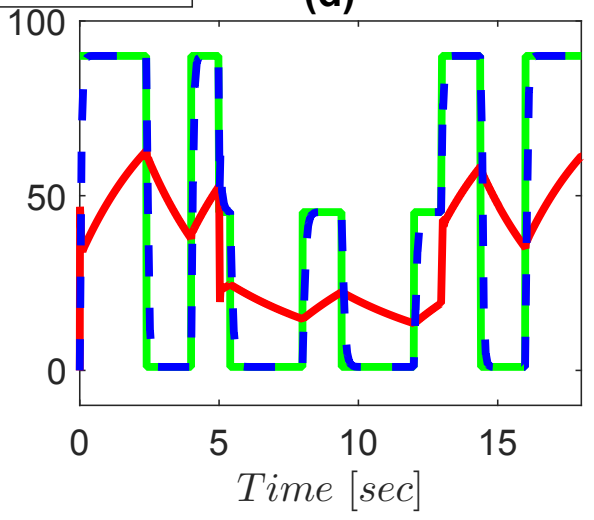

Figure 7.15: Simulation results of comparing the baseline second order DSMC with the baseline first order DSMC for tracking the desired throttle position, (a) $T=1 \mathrm{~ms}, n=16 \mathrm{bit}$, (b) $T=2 \mathrm{~ms}, n=16 \mathrm{bit}$, (c) $T=$ $5 \mathrm{~ms}, n=16 \mathrm{bit}$, and (d) $T=10 \mathrm{~ms}, n=16 \mathrm{bit}$.

\subsection{Summary and Conclusion}

The discrete sliding mode control formulation from Chapter 4 was used to design a position controller for an air throttle body under sampling and quantization imprecisions. The new design uses an online mechanism to predict and propagate ADC imprecisions. The robust DSMC was experimentally tested for an engine electronic throttle position tracking control problem with highly nonlinear dynamics. First, a nonlinear model was developed to simulate the throttle nonlinear dynamics. The 
parameters of the model were identified by applying a nonlinear least square technique on the experimental data collected from the throttle body experimental setup. Next, the validated physics-based model of the throttle was employed to formulate the baseline and robust DSMCs. Both controllers were experimentally tested on the throttle body setup developed in this study. The experimental and simulation results show:

- The developed switching nonlinear throttle model is able to predict the throttle dynamics with less than $1.5^{\circ}$ error.

- The proposed mechanism for predicting and propagating ADC imprecisions is viable for real-time operation, and can predict the uncertainties accurately, with an estimation error less than $0.01^{\circ}$.

- For the baseline DSMC, when the sampling time increases from $1 \mathrm{~ms}$ to $2 \mathrm{~ms}$, the tracking error increases by more than 5 times. Additionally, when the sampling time becomes $5 \mathrm{~ms}$, the tracking error increases by more than 14 times. In a similar manner to the sampling time, once the quantization level of the baseline DSMC changes from 10-bit to 6-bit, the tracking error grows by up to $80 \%$, compared to the case with no quantization.

- While the baseline DSMC tracking performance is considerably affected by the ADC imprecisions, upon incorporation of the predicted ADC uncertainties into the DSMC, the robustness against ADC uncertainties significantly improves. When the sampling time is increased from $1 \mathrm{~ms}$ to $5 \mathrm{~ms}$, and quantization level is decreased from 16 bit to 10 bit, the experimental test results showed that the 
robust DSMC can reduce the tracking errors of the baseline DSMC by up to 95\%. Similarly, once the sampling time is increased to $10 \mathrm{~ms}$, and quantization level is decreased to 6 bit, the robust DSMC showed better tracking performance by upto $60 \%$, compared to the baseline DSMC.

- The simulation test results showed the higher robustness characteristics of the second order DSMC against ADC imprecisions, compared to the baseline first order DSMC. It was observed that the second order DSMC can improve the tracking results of the equivalent first order DSMC by $60-90 \%$, in terms of the mean tracking errors. 


\section{Chapter 8}

\section{Conclusion and Future Work}

\subsection{Summary and Conclusion}

This PhD dissertation aspired to bridge the gap between the designed and implemented controllers with focus to overcome implementation and model uncertainties. It was shown that this gap can be filled by (i) modeling and quantification of uncertainties that arise from digital controller implementation imprecisions, (ii) design of robust controllers to overcome implementation imprecisions, and (iii) development of an adaptive control framework to mitigate the modeling uncertainties. The final outcome from this $\mathrm{PhD}$ dissertation is an uncertainty/imprecisions-adaptive, easily verifiable, and robust control theory framework. This goal has been achieved by completing the following seven stages. Summary of the results and contributions from each of these seven stages are listed below:

- Stage I (Chapter 2) - Early model-based controller verification and validation using reduced order controller design and studying the impact of implementation imprecisions on controller performance: 
1. A novel nonlinear model/controller (SMC) order reduction technique based on singular perturbation approximation (SPA) was developed and tested successfully for the automotive cold start emission control challenge in an SI engine. This resulted in simplification of the SMC design and reduced the computation costs.

2. The results of the reduced order SMC were compared with those from a full order SMC. The simulation results showed that the designed reduced order SMC outperforms the full order SMC in terms of engine-out and tailpipe $\mathrm{HC}$ emission with about $12 \%$ reduction in $\mathrm{HC}$ tailpipe emission. This is because the full order SMC treats all the control actuations equally. However, the reduced order SMC puts an optimized weight among the three control actuations since it evaluates the importance of affecting dynamics through the SPA model order reduction technique. Overall, the developed SPA approach not only simplified the original cold start model, but also helped the control design to better adjust priority among different control actuations.

3. An early model-based controller verification and validation (V\&V) was proposed and illustrated to study the effects of implementation imprecisions. including sampling time, quantization level, and fixed-point computations on the controller tracking performance. The proposed model-based controller design and verification approach can reduce the development time 
and cost for automotive controllers.

- Stage II (Chapter 3) -Easily verifiable uncertainty-adaptive sliding mode controller design to improve robustness against implementation imprecisions:

1. An uncertainty-adaptive SMC design methodology, based on the Lyapunov stability analysis, was developed to improve the SMC tracking performance under sampling and quantization imprecisions. The proposed control framework aimed to reduce the debugging efforts in the V\&V cycle by improving the controller robustness against implementation imprecisions.

2. The proposed single-input single-output (SISO) uncertainty-adaptive SMC framework was examined on the engine control problem introduced in Chapter 2. The uncertainty-adaptive SISO SMC showed better performance in exhaust temperature control by up to $50 \%$ reduction in the tracking error. However, due to the complexity of the engine plant, the developed adaptive SISO SMC could only provide small improvement (up to $16 \%$ and $2 \%$ ) in tracking the engine speed and air-to-fuel (AFR) ratio.

3. The SISO uncertainty-adaptive SMC was converted to a multi-input multioutput (MIMO) SMC using nonlinear balanced realization which transforms the physical state space to a non-physical space in which the states are prioritized according to their energy level.

4. The new uncertainty-adaptive MIMO SMC is able to improve the tracking errors for engine speed and AFR up to $41 \%$ and $22 \%$, respectively. The 
real-time testing of the MIMO SMC on a processor-in-the-loop (PIL) platform showed that the new adaptive controller is able to meet the control targets under ADC imprecisions, while the baseline SMC fails to meet the targets due to impacts of implementation imprecisions.

- Stage III (Chapter 4) - ADC uncertainty prediction and propagation models to design discrete sliding mode controllers (DSMC) with predicted implementation imprecisions:

1. An online methodology was developed for predicting and propagating the sampling and quantization imprecisions on both measured and control signals based on DSMC formulation. An analytical approach was proposed to propagate the imprecisions from measured signals to control signals, while for the nonlinear systems, an experimental approach was proposed to enable real-time uncertainty propagation.

2. The proposed prediction mechanism for sampling and quantization imprecisions was tested in real-time for the nonlinear combustion engine model (from Chapter 2), and also a linear model of a DC motor. The results verified the high accuracy of the uncertainty prediction technique.

3. The robustness characteristics of a conventional DSMC formulation was improved against ADC imprecisions by incorporating the predicted sampling and quantization imprecisions. This also helps to avoid conservative controller design.

4. The modified DSMC with predicted ADC imprecisions was designed and 
evaluated in real-time (by using the PIL setup from Chapter 3 ) for the SI engine and DC motor control problems under different sampling times and quantization levels.

5. Real-time testing results for both case studies showed that the new DSMC design is able to improve the tracking performance under sampling and quantization imprecisions by $40-90 \%$ compared to a conventional SMC design.

- Stage IV (Chapter 5)- Design of a first order adaptive DSMC with robustness to implementation and model uncertainties:

1. The DSMC with predicted ADC imprecisions from Chapter 4 was examined under multiplicative and additive types of model uncertainties. It was shown that the controller performance is sensitive to these uncertainties in the modeled dynamics. A discrete Lyapunov argument was employed to design adaptation laws to compensate for the unknown uncertainty terms in the controller structure.

2. The proposed adaptation laws are computationally efficient for real-time operation. The adaptation laws are able to quickly remove the errors in the modeled dynamics. Increasing the sampling time raises the required time for the adaptation laws to remove the errors in the model; however, these errors can be removed permanently. For the engine case study, all the additive and multiplicative unknown parameters were resolved within 
$2 \sec ($ sampling time $=10 \mathrm{~ms})$.

3. When the sampling time is increased from $10 \mathrm{~ms}$ to $35 \mathrm{~ms}$, the tracking errors from the baseline adaptive DSMC increase by 5-10 times. Additionally, by changing the ADC quantization level from 10 bit to 6 bit, the tracking errors of the baseline controller rise by up to 10 times. Incorporating the predicted $\mathrm{ADC}$ imprecisions into the adaptive DSMC improves the tracking errors by 50-60\% compared to the baseline controller.

4. Multiplicative and additive unknown parameters within the engine and DC motor control problems were considered to represent any mismatches in the modeled plants' dynamics. Although the baseline DSMCs are too sensitive to any errors in the models and fails under these mismatches, the proposed adaptation technique provides an effective tool for permanent removal of the errors due to unknown parameters. The proposed adaptation laws were derived via the stability analysis of the closed loop system which not only remove the errors in the model, but also guarantees the asymptotic stability of the controller. The adaptive robust DSMC is able to enhance the trajectory tracking errors by more than 95\%, compared to the nonadaptive DSMC, and removes the errors in the engine dynamics by up to $98 \%$.

- Stage V (Chapter 6) - Design of a second order MIMO adaptive DSMC with predicted implementation imprecisions: 
1. A new adaptive second order DSMC formulation for MIMO nonlinear and linear uncertain systems, along with an adaptation mechanism and a new switching control input, was presented in Chapter 6. First, the adaptation law, for handling the uncertainties within the model, was driven based on a discrete Lyapunov stability theorem. Second, the behavior of the second order DSMC was studied on both reaching and sliding phases. In order to ensure the controller robustness against external ADC imprecisions, a new switching control input was introduced, which contains the knowledge of ADC imprecisions via an online sampling and quantization uncertainties prediction and propagation mechanism. Third, the asymptotic stability of the proposed controller was guaranteed by invoking the new Invariance Principal for nonlinear discontinuous systems.

2. The proposed controller was evaluated for the nonlinear combustion engine (Chapter 2) and linear DC motor (Chapter 4) control problems. The designed second order adaptive MIMO/SISO DSMC was tested in realtime on a real ECU inside a PIL setup.

3. The second order DSMC showed higher robustness against ADC uncertainties. For the engine case study, when the sampling rate was changed from $20 \mathrm{~ms}$ to $80 \mathrm{~ms}$, and the quantization level changed from 16-bit to 10-bit, the second order DSMC was able to improve the tracking errors by more than $90 \%$ compared to the first order DSMC. For the DC motor case, up to $84 \%$ improvements were achieved by using the MIMO second order 
DSMC compared to the SISO first order DSMC, under ADC uncertainties.

4. Inclusion of the physical coupling within the engine dynamics in the controller structure via the MIMO formulation allows for further improvement in $A F R$ and $T_{\text {exh }}$ controllers tracking performance (by up to $46 \%$ and $11 \%$, respectively). However, the engine speed seems to lean strongly toward a decoupled or minimally coupled structure. On the other side, it was observed that by utilizing the MIMO structure for the DC motor controller, which takes into account the dynamics coupling in the controller actions, the performance of the adaptive second order MIMO DSMC with ADC uncertainties is improved by 40-60\%, compared to the adaptive SISO second order DSMC.

5. In the presence of model uncertainties, it was shown that the proposed adaptation mechanism is able to remove the errors in the engine and DC motor models permanently and quickly (by up to 90\%). Moreover, compared to the SISO DSMC, the MIMO engine controller shows faster unknown parameters convergence rates for the $A F R$ and $T_{\text {exh }}$ controllers. The MIMO adaptive second order DSMC is able to improve the $A F R$ and $T_{\text {exh }}$ tracking errors by $43 \%$ and $33 \%$, respectively, compared to the SISO second order adaptive DSMC under modeling and ADC uncertainties.

- Stage VI - Design and build PIL platforms for real-time testing of the proposed control algorithms from Chapter 3, 4, 5, and 6, 
1. A modular processor-in-the-loop (PIL) powertrain simulator was designed and built. This included design of hardware configuration, selection of the components according to the research requirements, procurement of the components, and PIL setup installation and integration.

2. The designed PIL setup is able to do the processing real-time, read and send analog/digital signals through configurable I/O ports, and communicate with real control unit via standard communication protocols. Two processors are employed in the PIL setup. The first one is a National Instrument (NI) PXI processor (NI PXIe-8135) in which the engine plant model is implemented. The second processor is a dSPACE MicroAutobox II (MABX) which is the main ECU.

3. The application of the PIL setup is to verify the performance of the real control units. It means that the control algorithms can be implemented on real control units, and their functionality can be tested using PIL setup under different levels of implementation and modeling uncertainties, prior to the test on the vehicle platform.

- Stage VII (Chapter 7) - Experimental validation of the first order DSMC with predicted implementation imprecisions on an electronic air throttle body test setup:

1. A nonlinear physics-based model was developed and identified for an electronic throttle body with respect to experimental data. The developed nonlinear model is able to predict the throttle dynamics with less than 
$1.5^{\circ}$ error.

2. Based on the developed model, the new DSMC formulation from Chapter 4 was used to design a position controller for the throttle. The designed controller with and without the incorporated ADC imprecisions was experimentally tested on the throttle testbed. The test results showed that the tracking performance of the DSMC with no incorporated ADC impreciseness knowledge is considerably affected by the ADC imprecisions

3. Upon incorporation of the predicted ADC uncertainties into the DSMC, the robustness against ADC uncertainties significantly improves. When the sampling time was increased from $1 \mathrm{~ms}$ to $5 \mathrm{~ms}$, and quantization level was decreased from 16 bit to 10 bit, the experimental test results showed that the robust DSMC could reduce the tracking errors of the baseline DSMC by up to $95 \%$. Similarly, once the sampling time was increased to $10 \mathrm{~ms}$, and quantization level was decreased to 6 bit, the robust DSMC showed better tracking performance by upto $60 \%$, compared to the baseline DSMC. Overall, the simulation and experimental test results proved that the designed robust DSMC with predicted ADC uncertainties can enhance the throttle position tracking errors under various digital implementation conditions compared to the baseline DSMC by up to $70 \%$ on average. 


\subsection{Suggestions for Future Work}

The results and outcomes of this $\mathrm{PhD}$ dissertation may be extended in the future works via the following research areas:

- The proposed first and second order DSMCs in this study, which were designed and tested for the engine cold start emission problem, could be experimentally implemented on the actual engine setup.

- The adaptive DSMC framework in this study considers the uncertainty and variation within the plant model $(f)$. The adaptation technique could be extended to address uncertainties in the control input functions ( $g$ in Eq. 4.15)).

- The robustness of the conventional DSMC needs to be investigated and improved under measurement noise. The analog and digital signals before ADC are subject to measurement noise which can increase the imprecisions at the controller inputs and outputs (I/O) significantly, if they are not handled effectively downstream of the ECU input.

- The proposed adaptation laws for handling multiplicative and additive types of modeling uncertainties could be extended to nonlinear/linear systems with time-varying unknown uncertainty terms.

- The concept of higher order sliding modes for discrete-time systems could be extended to develop a predictive sliding mode control theory with lower optimization costs. 
- For the throttle body position DSMC design, future works may focus on: (i) inclusion of uncertainties in the throttle plant model to address the model parameter variations over temperature and time via the first and second order adaptive DSMCs proposed in Chapter 5 and 6, and (ii) developing a highfidelity model to extend the operation range of the model-based controller and test the controller against various desired throttle position profiles including ramp trajectories.

- The proposed model-based DSMC design in this study is generic and could be applied to various challenging automotive control systems. 


\section{References}

[1] M. Shahbakhti, M.R. Amini, J. Li, S. Asami, and J.K. Hedrick. Early ModelBased Design and Verification of Automotive Control System Software Implementations. J. of Dynamic Sys., Measurement, and Control, 137(2):021006, 2015.

[2] M. R. Amini, M. Shahbakhti, and A. Ghaffari. A Novel Singular Perturbation Technique for Model-based Control of Cold Start Hydrocarbon Emission. SAE Int. J. of Engines, 7(3):1290-1301, 2014.

[3] M. R. Amini, M. Shahbakhti, and J. K. Hedrick. Easily Verifiable Adaptive Sliding Mode Controller Design with Application to Automotive Engines. 2016. 16 pages, SAE Technical Paper 2016-01-0629.

[4] M. R. Amini, M. Shahbakhti, and J. K. Hedrick. Discrete Sliding Controller Design with Robustness to Implementation Imprecisions via Online Uncertainty Prediction. 2016 American Control Conference, 2016. 6 Pages, Boston, MA, USA. 
[5] M. R. Amini, M. Shahbakhti, S. Pan, and J. K. Hedrick. Handling Model and Implementation Uncertainties via An Adaptive Discrete Sliding Controller Design. ASME 2016 Dynamic Systems and Control Conference, 2016. 10 Pages, Minneapolis, MN, USA .

[6] M.R. Amini, M. Shahbakhti, S. Pan, and J.K. Hedrick. Bridging the Gap Between Designed and Implemented Controllers via Adaptive Robust Discrete Sliding Mode Control. Control Engineering Practice, 59:1-15, 2017.

[7] R. Salehi, M. Shahbakhti, and J. K. Hedrick. Hybrid Switching Control of Automotive Coldstart Hydrocarbon Emission. 2013 American Control Conference, 2013. Washington, DC, USA.

[8] M. R. Amini, M. Shahbakhti, S. Pan, and J. K. Hedrick. Discrete Adaptive Second Order Sliding Mode Controller Design with Application to Automotive Control Systems with Model Uncertainties. 2017 American Control Conference, 2017. 6 Pages, Seattle, WA, USA.

[9] M. R. Amini, Razmara, and M. M. Shahbakhti. Robust Model-Based Discrete Sliding Mode Control of an Automotive Electronic Throttle Body. SAE International Journal of Commercial Vehicles, 10(1), 2017. 14 Pages.

[10] A. Gray, T. Lin, J. K. Hedrick, H. E. Tseng, and F. Borrelli. Predictive Control for Agile Semi-Autonomous Ground Vehicle using Motion Primitives. 2010 American Control Conference, 2012. Montreal, Canada. 
[11] J. B. Dabney, G. Barber, and D. Ohi. Estimating Direct Return on Investment of Independent Verification and Validation using COCOMO-II. Proceedings of the 10th IASTED Int. Conference on Software Engineering and Applications, 2006. Dallas, TX, USA.

[12] M. Shahbakhti, J. Li, and J. K. Hedrick. Early Model-Based Verification of Automotive Control System Implementation. 2012 American Control Conference, 2012. Montreal, Canada.

[13] I. Karafyllis and C. Kravaris. Global Stability Results for Systems Under Sampled-data Control. Int. J. of Robust and Nonlinear Control, 19(10):11051128, 2009.

[14] S. Monaco and D. Normand-Cyrot. Advanced Tools for Nonlinear Sampleddata Systems' Analysis and Control. Int. J. of Robust and Nonlinear Control, $13(2): 221-241,2007$.

[15] E. Feron. From Control Systems to Control Software. IEEE Control Systems Magazine, 30(6):50-71, 2010.

[16] A. Anta, R. Majumdar, I. Saha, and P. Tabuada. Automatic Verification of Control System Implementations. Proc. of the 10th ACM int. conf. on Embedded software, 2010. Scottsdale, AZ, USA.

[17] A.R. Comeau and N. Hori. State-space Forms for Higher Order Discrete-time Models. System Control Letter, 34(1):23-31, 1998. 
[18] M. Rupak, I. Saha, and M. Zamani. Synthesis of Minimal-error Control Software. 10th ACM Int. Conf. on Embedded software, 2012. Tampere, Finland.

[19] D. Williamson. Finite Wordlength Design of Digital Kalman Filters for State Estimation. IEEE Trans. on Automatic Control, 30(10):930-939, 1985.

[20] D. Williamson. Optimal Finite Wordlength Linear Quadratic Regulation. IEEE Trans. on Automatic Control, 34(12):1218-1228, 1989.

[21] K. Liu, R. E. Skelton, and K. Grigoriadis. Optimal Controllers for Finite Wordlength Implementation. IEEE Trans. on Automatic Control, 37(9):12941304, 1992.

[22] J. A. Lopez, C. Carreras, and O. Nieto-Taladriz. Interval-based Characterization of Fixed-point LTI Systems with Feedback Loops. IEEE Trans. on CAD of Integ. Circ. and Sys., 26(11):1923-1932, 2007.

[23] K. Edelberg, M. Shahbakhti, and J. K. Hedrick. Incorporation of Implementation Impercesion in Automotive Control Design. 2013 American Control Conference, 2013. Washington, DC, USA.

[24] K. Edelberg, S. Pan, and J. K. Hedrick. Design of Automotive Control Systems Robust to Hardware Impersicion. ASME 2013 Dynamic Systems and Contol Conference, 2013. Palo Alto, CA, USA.

[25] T. Hagiwara and M. Araki. FR Operator Approach for the H2 Analysis and 
Synthesis of Sampled Data Systems. IEEE Trans. on Automatic Control, 40(8):1411-1421, 1995.

[26] L. Grüne and D. Nesic. Optimization Based Stabilization of Sampled Data Nonlinear Systems via Their Approximate Discrete-time Models. SIAM Journal on Control and Optimization, 42(1):98-122, 2003.

[27] H. K. Khalil. Performance Recovery under Output Feedback Sampled-Data Stabilization of a Class of Nonlinear Systems. IEEE Trans. on Automatic Control, 49(12):2173-2184, 2004.

[28] T. Ieko, Y. Ochi, and K. Kanai. New Design Method for Pulse-Width Modulation Control Systems via Digital Redesign. J. of Guidance, Control, and Dynamics, 22(1):123-128, 1999.

[29] S. Monaco, D. Normand-Cyrot, and S. Stornelli. On the Linearizing Feedback in Nonlinear Sampled Data Control Schemes. 25th IEEE Conference on Decision and Control, 1986. Athens, Greece.

[30] S. Monaco and D. Normand-Cyrot. On the Sampling of a Linear Control System. 24th IEEE Conference on Decision and Control, 1985. Fort Lauderdale, FL, USA.

[31] V. Veliov. On the Time Discretization of Control Systems. SIAM Journal on Control and Optimization, 35(5):1470-1486, 1997. 
[32] S. Monaco, D. Normand-Cyrot, and T. Isola. Nonlinear Decoupling in Discrete System. IFAC Symposium on Nonlinear Control System Design, 1989. Capri, Italy.

[33] S. Monaco and D. Normand-Cyrot. An Introduction to Motion Planning Under Multirate Digital Control. 31st IEEE Conference on Decision and Control, 1992. Tucson, AZ, USA.

[34] S. Monaco and D. Normand-Cyrot. Issues on Nonlinear Digital Systems. European Journal of Control, 7(2):160-178, 2001.

[35] S. Di Gennaro, S. Monaco, and D. Normand-Cyrot. Nonlinear Digital Scheme for Attitude Tracking. Journal of Guidance, Control, and Dynamics, 22(3):467478, 1999.

[36] J. P. Keller and B. Anderson. A New Approach to the Discretization of Continuous-time Controllers. IEEE Trans. on Automatic Control, 37(2):214224, 1992.

[37] L. Grüne, K. Worthmann, and D. Nesic. High Order Approximations by Sampled-data Feedback. 17th International Symposium on Mathematical Theory of Networks and Systems, 2006. Kyoto, Japan.

[38] J. W. Grizzle and P. V. Kokotovic. Feedback Linearization for Sampled-data Systems. IEEE Trans. on Automatic Control, 33(9):857-859, 1988. 
[39] N. Kazantzis and C. Kravaris. System-theoretic Properties of Sampled-data Representations of Nonlinear Systems Obtained via Taylor Lie Series. International Journal of Control, 67(6):997-1020, 1997.

[40] D. Nesic and L. Grüne. Lyapunov-based Continuous-time Nonlinear Controller Redesign for Sampled-data Implementation. Automatica, 41(7):1143$1156,2005$.

[41] L. Zaccarian, A.R. Teel, and D. Nesic. On Finite Gain Lp Stability of Nonlinear Sample-data Systems. Systems and Control Letters, 49(3):201-212, 2003.

[42] C. M. Kellett, H. Shim, and A. R. Teel. Further Results on Robustness of (Possibly Discontinuous) Sample and Hold Feedback. IEEE Trans. on Automatic Control, 49(7):1081-1089, 2004.

[43] D. S. Laila, D. Nesic, and A. R. Teel. Open and Closed-loop Dissipation Inequalities under Sampling and Controller Emulation. European Journal of Control, 8(2):109-125, 2002.

[44] L. Grüne. Homogeneous state Feedback Stabilization of Homogenous Systems. SIAM J. on Control and Opt., 38(4):1288-1308, 2000.

[45] G. Herrmann, S. K. Spurgeon, and c. Edwards. Discretization of Sliding Mode Based Control Schemes. 38th Conference on Decision and Control, 1999. Phoenix, AZ, USA. 
[46] D. S. Bernstein and C. V. Hollot. Robust Stability for Sampled-data Control Systems. Systems and Control Letters, 13(3):217-226, 1989.

[47] E.D. Sontag. "Mathematical Control Theory". Springer: New York, NY, 1991. Chapter 2, 2nd ed.

[48] D. Nesic, A. R. Teel, and E. D. Sontag. Formulas Relating KL Stability Estimates of Discrete-time and Sampled-data Nonlinear Systems. Systems and Control Letters, 38(1):49-60, 1999.

[49] B. Hu and A. Michel. Stability Analysis of Digital Control Systems with TimeVarying Sampling Periods. Automatica, 36(6):897-905, 2000.

[50] H. Ye, A. N. Michel, and L. Hou. Stability Theory for Hybrid Dynamical Systems. IEEE Trans. on Automatic Control, 43(4):461-474, 1988.

[51] Z. Artstein and G. Weiss. State Nullification by Memoryless Output Feedback. Mathematics of Control, Signals and Systems, 17(1):38-56, 2005.

[52] S. Monaco and D. Normand-Cyrot. Zero Dynamics of Sampled Nonlinear Systems. Systems and Control Letters, 11(3):229-234, 1988.

[53] S. Monaco and D. Normand-Cyrot. A Unified Representation for Nonlinear Discrete-time and Sampled Dynamics. Journal of Mathematical Systems, Estimation, and Control, 7(4):477-503, 1997. 
[54] L. Grune. Input-to-state Dynamical Stability and Its Lyapunov Function Characterization. IEEE Trans. on Auto. Control, 47(9):1499-1504, 2002.

[55] S. Asami, A. Cranmer, M. Shahbakhti, and J. K. Hedrick. Model-Based Control Via Balanced Realization For Automotive Cold Start Hydrocarbon Reduction. ASME 2011 Dynamic Systems and Contol Conference, 2011. Arlington, TX, USA.

[56] E. Misawa. Discrete-time sliding mode control for nonlinear systems with unmatched uncertainties and uncertain control vector. ASME J. of Dynamic System, Measurement, and Control, 119(3):503-512, 1997.

[57] J.-J.E. Slotine and L. Weiping. "Applied nonlinear control". Prentice-hall Englewood Cliffs, NJ, Chapter 7 \& 8, 1991.

[58] C. Chan. Discrete adaptive sliding-mode tracking controller. Systems \& Control Letters, 33(5):999-1002, 1997.

[59] S. Pan. Discrete Sliding Control for the Dynamics of Engine Cold Start. PhD thesis, University of California Berkeley, 2014.

[60] D. Muñoz and D. Sbarbaro. An adaptive sliding-mode controller for discrete nonlinear systems. IEEE Transactions on Industrial Electronics, 47(3):574-581, 2000. 
[61] Y. Fang, T. Chow, and X. Li. Use of a recurrent neural network in discrete sliding-mode control. IEEE Proceedings-Control Theory and Applications, 146(1):84-90, 1999.

[62] S. Pan and J. K. Hedrick. Tracking Controller Design for MIMO Nonlinear Systems with Application to Automotive Cold Start Emission Reduction. Journal of Dynamic Systems, Measurement, and Control, 137(10):101013, 2015.

[63] A. Cranmer, M. Shahbakhti, and J. K. Hedrick. Grey-box Modeling Architectures for Rotational Dynamic Control in Automotive Engines. 2012 American Control Conference, 2012. Montreal, Canada.

[64] B. Moore. Principal Component Analysis in Linear Systems: Controllability, Observability, and Model Reduction. IEEE Transaction on Automatic Control, 26(1):17-32, 1981.

[65] Y. Liu and B. D. O. Anderson. Singular Perturbation Approximation of Balanced Systems. Int. Journal of Control, 50(40):1379-1405, 1989.

[66] P. Kokotovic, R.E. O’Malley, and P. Sannuti. Singular Perturbations and Order Reduction in Control Theory-an Overview. International Journal of Robust and Nonlinear Control, 19(10):1105-1128, 2009.

[67] K. J. Mitts, K. Lang, T. Roudier, and D. L. Kiskis. Using a Co-simulation Framework to Enable Software-in-the-Loop Powertrain System Development. 2009. SAE Technical Paper 2009-01-0520. 
[68] V. Jaikamal. Model-based ECU Development-An Integrated MiL-SiL-HiL Approach. 2009. SAE Technical Paper 2009-01-0153.

[69] A. Vora, H. Wu, C. Wang, Y. Qian, G. Shaver, V. Motevalli, P. Meckl, O. Wasynczuk, and H. Zhang. Development of a SIL, HIL and Vehicle TestBench for Model-Based Design and Validation of Hybrid Powertrain Control Strategies. 2014. SAE Technical Paper 2014-01-1906.

[70] A. Taghavipour, R. Masoudi, N. L. Azad, and J. McPhee. High-Fidelity Modeling of a Power-Split Plug-In Hybrid Electric Powertrain for Control Performance Evaluation. ASME 2013 Int. Design Eng. Technical Conferences and Computers and Information in Eng. Conference, 2013. Portland, OR, USA.

[71] I. Saha. Closing the Gap in Control System Implementations. PhD thesis, University of California Berkeley, 2013.

[72] J. Kapinski, A. Donzé, F. Lerda, H. Maka, S. Wagner, and B. H. Krogh. Control Software Model Checking Using Bisimulation Functions for Nonlinear Systems. 47th IEEE Conference on Decision and Control, 2008. Cancun, Mexico.

[73] M. Althoff, C. Le Guernic, and B. H. Krogh. Reachable Set Computation for Uncertain Time-Varying Linear Systems. Proceedings of the 14th int. conference on Hybrid systems: computation and control, 2011. Chicago, IL, USA.

[74] M. Althoff and J. M. Dolan. Online Verification of Automated Road Vehicles Using Reachability Analysis. IEEE Transaction on Robotics, 2014. In Press. 
[75] J. Park, A. Kurt, and U. Ozguner. Hybrid Systems Modeling and ReachabilityBased Controller Design Methods for Vehicular Automation. Unmanned Systems, 2(2):101-119, 2014.

[76] B. Murphy, A. Wakefield, and J. Friedman. Best Practices for Verification, Validation, and Test in Model-Based Design. 2008. SAE Technical Paper 2008$01-1469$.

[77] T. Erkkinen and M. Conrad. Verification, Validation, and Test with ModelBased Design. 2008. SAE Technical Paper 2008-01-2709.

[78] A. H. Khan, Z. H. Khan, and Z. Weiguo. Model-Based Verification and Validation of Safety-Critical Embedded Real-Time Systems: Formation and Tools. In Embedded and Real Time System Development: A Software Engineering Perspective, volume 520 of Studies in Computational Intelligence, pages 153-183. Springer Berlin Heidelberg, 2014.

[79] H. Eldib and C. Wang. An SMT Based Method for Optimizing Arithmetic Computations in Embedded Software Code. Formal Methods in ComputerAided Design (FMCAD2013), 2013. Portland, OR, USA.

[80] K. Lang and M. Kropinski. Virtual Powertrain Calibration at GM Becomes a Reality. 2010. SAE Technical Paper 2010-01-2323.

[81] R. Diewald, T. Cartus, M. Schüßler, and H. Bachler. Model Based Calibration Methodology. 2009. SAE Technical Paper 2009-01-2837. 
[82] B. Bielaczyc and J. Merkisz. EURO III/EURO IV Emissions - A Study of Cold Start and Warm up Phases with a SI Engine. 1999. SAE Technical Paper 1999-01-1073.

[83] M. Weilenmann, J. Favez, and R. Alvarez. Cold-start Emissions of Modern Passenger Cars at Different Low Ambient Temperatures and Their Evolution over Vehicle Legislation Categories. J. of Atmospheric Environmen, 43(15):24192429, 2009.

[84] B. T. Shaw II. Modelling and Control of Automotive Coldstart Hydrocarbon Emissions. PhD thesis, UC Berkeley, 2002.

[85] P. R. Sanketi, J. C. Zavala, J. K. Hedrick, M. Wilcutts, and T. Kaga. A Simplified Catalytic Converter Model for Automotive Coldstart Applications with Adaptive Parameter Fitting. 8th International Symposium on Advanced Vehicle Control, 2006. Taipei, Taiwan.

[86] P. R. Sanketi. Coldstart Modeling and Optimal Control Design for Automotive SI Engines. PhD thesis, University of California Berkeley, 2009.

[87] M. Roopaei and V. E. Balas. Adaptive Gain Sliding Mode Control in Uncertain MIMO Systems. 3rd International Workshop on Soft Computing Applications, 2009. Szeged, Hungary and Arad, Romania.

[88] A. Hansen, M. Shahbakhti, and J. K. Hedrick. Impact of Implementation Impercision on Sliding Mode Controller Design for Automotive Cold Start Emissions. 
ASME 2012 Dynamic Systems and Control Conference, 2012. Fort Lauderdale, FL, USA.

[89] K. Edelberg, S. Pan, and J. K. Hedrick. A Discrete-Time Sliding Mode Formulation for Automotive Cold-Start Emission Control. 52nd IEEE Conference on Decision and Control, 2013. Florence, Italy.

[90] B. Messner and D. Tilbury. Control Tutorials for MATLAB and Simulink. library.cmu.edu/ctms, 1998.

[91] D. Efimov, A. Polyakov, L. Fridman, W. Perruquetti, and J. Richard. Delayed Sliding Mode Control. Automatica, 64:37-43, 2016.

[92] X. Zhang, H. Su, and R. Lu. Second-Order Integral Sliding Mode Control for Uncertain Systems with Control Input Time Delay Based on Singular Perturbation Approach. IEEE Transactions on Automatic Control, 60(11):3095-3100, 2015.

[93] P. Yang, J. Ni, X. Pan, and J. Liu. Global Robust Sliding Mode Control for Time-Delay Systems with Mismatched Uncertainties. Circuits, Systems, and Signal Processing, pages 1-12, 2015.

[94] I. Barkana. The New Theorem of Stability-Direct Extension of Lyapunov Theorem. Mathematics in Engineering, Science \&5 Aerospace (MESA), 6(3), 2015. 
[95] V. I. Utkin. "Sliding Modes in Control and Optimization". Springer Berlin Heidelberg, Chapter 4, 1992.

[96] F. Nollet, T. Floquet, and W. Perruquetti. Observer-based second order sliding mode control laws for stepper motors. Control engineering practice, 16(4):429443, 2008.

[97] V. Acary and B. Brogliato. Implicit Euler numerical scheme and chattering-free implementation of sliding mode systems. Systems $\& 6$ Control Letters, 59(5):284$293,2010$.

[98] O. Huber, V. Acary, B. Brogliato, and F. Plestan. Implicit Discrete-Time Twisting Controller without Numerical Chattering: Analysis and Experimental Results. Control Engineering Practice, 46:129-141, 2016.

[99] M. Mihoub, A. Nouri, and R. Abdennour. Real-Time Application of Discrete Second Order Sliding Mode Control to a Chemical Reactor. Control Engineering Practice, 17(9):1089-1095, 2009.

[100] T. Salgado-Jimenez, J. Spiewak, P. Fraisse, and B. Jouvencel. A Robust Control Algorithm for AUV: Based on a High Order Sliding Mode. OCEANSO4. MTTS/IEEE TECHNO-OCEAN04, 2004. Kobe, Japan.

[101] H. Sira-Ramirez. Structure at infinity, zero dynamics and normal forms of systems undergoing sliding motions. International Journal of Systems Science, 21(4):665-674, 1990. 
[102] W. Gao, Y. Wang, and A. Homaifa. Discrete-Time Variable Structure Control Systems. IEEE Transactions on Industrial Electronics, 42(2):117-122, 1995.

[103] L. Xiao and Y. Zhu. Higher Order Sliding Mode Prediction Based Discrete-Time Variable Structure Control for Nonlinear Uncertain Systems. International Conference on Computer Science and Service System (CSSS), 2011. Nanjing, China.

[104] I. Barkana. Defending the Beauty of the Invariance Principle. International Journal of Control, 87(1):186-206, 2014.

[105] H. Khalil and J. Grizzle. "Nonlinear Systems". Prentice-hall Englewood Cliffs, NJ, Chapter 13, 1996.

[106] M. S. Branicky. Multiple Lyapunov Functions and Other Analysis Tools for Switched and Hybrid Systems. IEEE Transactions on Automatic Control, 43(4):475-482, 1998.

[107] B. Lu and F. Wu. Switching LPV Control Designs Using Multiple ParameterDependent Lyapunov Functions. Automatica, 40(11):1973-1980, 2004.

[108] D. Chatterjee and D. Liberzon. Stability Analysis of Deterministic and Stochastic Switched Systems via a Comparison Principle and Multiple Lyapunov Functions. SIAM Journal on Control and Optimization, 45(1):174-206, 2006.

[109] A. Bartoszewicz. Remarks on discrete-time variable structure control systems. IEEE Transactions on Industrial Electronics, 43(1):235-238, 1996. 
[110] G. Monsees. Discrete-Time Sliding Mode Control. PhD thesis, Delft University of Technology, 2002.

[111] A. G. Stefanopoulou, J. S. Freudenberg, and J. W. Grizzle. Variable Camshaft Timing Engine Control. IEEE Transactions on Control Systems Technology, 8(1):23-34, 2000.

[112] A. G. Stefanopoulou, K. R. Butts, J. A. Cook, J. S. Freudenberg, and J. W. Grizzle. Consequences of Modular Controller Development for Automotive Powertrains: A Case Study. 34th IEEE Conference on Decision and Control, 1995. New Orleans, LA USA.

[113] S. Smith. "Digital Signal Processing: A Practical Guide for Engineers and Scientists". Newnes, Chapter 3, 2013.

[114] D. Pavković, J. Deur, M. Jansz, and N. Perić. Adaptive Control of Automotive Electronic Throttle. Control Engineering Practice, 14(2):121-136, 2006.

[115] J. Deur, D. Pavkovic, N. Peric, M. Jansz, and D. Hrovat. An Electronic Throttle Control Strategy Including Compensation of Friction and Limp-Home Effects. IEEE Transactions on Industry Applications, 40(3):821-834, 2004.

[116] M. Vasak, M. Baotic, I. Petrovic, and N. Peric. Hybrid Theory-Based TimeOptimal Control of an Electronic Throttle. IEEE Transactions on Industrial Electronics, 54(3):1483-1494, 2007. 
[117] M. Vašak, M. Baotić, M. Morari, I. Petrović, and N. Perić. Constrained Optimal Control of an Electronic Throttle. International Journal of Control, 79(05):465$478,2006$.

[118] C. de Witt, I. Kolmanovsky, and J. Sun. Adaptive Pulse Control of Electronic Throttle. 2001 American Control Conference, 2001. Arlington, VA USA.

[119] R. Loh, W. Thanom, J. Pyko, and A. Lee. Electronic Throttle Control System: Modeling, Identification and Model-Based Control Designs. Engineering, $5(07): 587,2013$

[120] X. Gong, Y. Hu, P. Sun, and H. Chen. A Nonlinear Feedforward-Feedback Controller Design for Electronic Throttle Based on Flatness. 24th Chinese Control and Decision Conference (CCDC), pages 1542-1547, 2012.

[121] M. Corno, M. Tanelli, S. M. Savaresi, and L. Fabbri. Design and Validation of a Gain-Scheduled Controller for the Electronic Throttle Body in Ride-byWire Racing Motorcycles. IEEE Transactions on Control Systems Technology, 19(1):18-30, 2011.

[122] S. Zhang, J. J Yang, and G. Zhu. LPV Gain-Scheduling Control of an Electronic Throttle with Experimental Validation. American Control Conference, pages 190-195, 2014.

[123] S. Zhang, J. J Yang, and G. Zhu. LPV Modeling and Mixed Constrained 
Control of an Electronic Throttle. IEEE/ASME Transactions on Mechatronics, 20(5):2120-2132, 2015.

[124] Y. Pan, U. Ozguner, and O. Dagci. Variable-Structure Control of Electronic Throttle Valve. IEEE Transactions on industrial electronics, 55(11):3899-3907, 2008.

[125] M. Reichhartinger and M. Horn. Robust Position Control of an Electromechanical Actuator for Automotive Applications. World Academy of Science, Engineering and Technology, 5(11):1184-1188, 2011.

[126] C. Rossi, A. Tilli, and A. Tonielli. Robust Control of a Throttle Body for Drive by Wire Operation of Automotive Engines. IEEE Transactions on control systems technology, 8(6):993-1002, 2000.

[127] U. Ozguner, S. Hong, and Y. Pan. Discrete-Time Sliding Mode Control of Electronic Throttle Valve. 40th IEEE Conference on Decision and Control, 2001. Orlando, FL USA.

[128] M. di Bernardo, A. di Gaeta, U. Montanaro, J. Olm, and S. Santini. Experimental Validation of the Discrete-Time MCS Adaptive Strategy. Control Engineering Practice, 21(6):847-859, 2013.

[129] K. Nakano, U. Sawut, K. Higuchi, and Y. Okajima. Modelling and ObserverBased Sliding-Mode Control of Electronic Throttle Systems. ECTI Transactions on Electrical Eng., Electronics, and Communications, 4(1):22-28, 2006. 
[130] R. Grepl and B. Lee. Modeling, Parameter Estimation and Nonlinear Control of Automotive Electronic Throttle Using a Rapid-Control Prototyping Technique. International Journal of Automotive Technology, 11(4):601-610, 2010.

[131] C. Garcia. Comparison of Friction Models Applied to a Control Valve. Control Engineering Practice, 16(10):1231-1243, 2008. 


\section{Appendix A}

\section{Parameters of the Models/Controllers}

\section{A.1 Parameters of cold start plant model}

\section{A.1.1 Constants}

Table A.1

Plant model constants.

\begin{tabular}{c|c}
\hline \hline Constant & Value [unit] \\
\hline$J$ & $0.1454\left[\mathrm{~m}^{2} \mathrm{~kg}\right]$ \\
$\tau_{f}$ & $0.06[1 / \mathrm{sec}]$ \\
$m C_{p}$ & $1250[\mathrm{~J} / \mathrm{K}]$ \\
$a$ & $-2[-]$ \\
$n$ & $110[-]$ \\
$\theta_{\text {evo }}$ & $110\left[{ }^{o} \mathrm{ATDC}\right]$ \\
$r_{c}$ & $9[-]$ \\
\hline \hline
\end{tabular}

\section{A.1.2 Functions}

$$
\begin{gathered}
S I=7.5 \Delta+600 \\
A I=\cos (0.13(A F R-13.5))
\end{gathered}
$$




$$
\begin{aligned}
& T_{E}=30000 m_{a}-0.4 \omega_{e}-100 \\
& \tau_{e}=2 \pi / \omega_{e} \\
& \dot{Q}_{i n}=16\left(T_{e x h}-T_{c a t}\right) \\
& \dot{Q}_{\text {out }}=0.642\left(T_{\text {cat }}-T_{\text {atm }}\right) \\
& \dot{Q}_{g e n}=22.53\left(\dot{m}_{a o}+\dot{m}_{f} T_{e x h}\right) \cdot \eta_{c a t} \cdot \dot{H} C_{e n g} \\
& \dot{m}_{a o}=0.0254 m_{a} \cdot \omega_{e} \cdot \eta_{v o l} \\
& \eta_{v o l}=m_{a}{ }^{2}\left(-0.1636 \omega_{e}^{2}-7.093 \omega_{e}-1750\right) \\
& +m_{a}\left(0.0029 \omega_{e}^{2}-0.4033 \omega_{e}+85.38\right) \\
& -\left(1.06 e-5 \omega_{e}^{2}-0.0021 \omega_{e}-0.2719\right) \\
& \theta_{0}=\Delta+10 \\
& \delta \theta= \begin{cases}0.1(16.2-A F R)^{2}+80 \quad A F R>A F R_{s t} \\
0.4(16.2-A F R)^{2}+80 \quad A F R \leq A F R_{s t}\end{cases}
\end{aligned}
$$




\section{A.2 Parameters of the Engine SMCs in Chapter 3}

\section{A.2.1 Uncertainty-Adaptive SISO SMC}

Table A.2

Gains $(\lambda, \gamma)$ and boundary layers $(\phi)$ of designed SISO adaptive SMC for the engine control.

\begin{tabular}{c|c|c|c|c}
\hline \hline & Controller & $\lambda$ & $\phi$ & $\gamma$ \\
\hline & $\Delta$ & 10 & 7 & 0.9 \\
& $\dot{m}_{f c}$ & 100 & $10^{-3}$ & 0.0025 \\
$m_{a, d}$ & 10 & 1 & 0.1 \\
& $\dot{m}_{a i}$ & 10 & $1.8 \times 10^{-5}$ & 0.15 \\
\hline \hline
\end{tabular}

\section{A.2.2 Uncertainty-Adaptive MIMO SMC}

Table A.3

Gains and boundary layers of designed MIMO adaptive SMC for the engine control.

\begin{tabular}{c|c|c|c}
\hline \hline Controller & $\lambda$ & $\phi$ & $\gamma$ \\
\hline MIMO Adaptive MSC & 100 & 0.1 & 0.9 \\
\hline \hline
\end{tabular}

\section{A.3 Parameters of DC Motor Plant Model}

Table A.4

DC motor model constants.

\begin{tabular}{c|c}
\hline \hline Constant & Value [unit] \\
\hline$J$ & $0.02\left[\mathrm{~m}^{2} \mathrm{~kg}\right]$ \\
$R$ & $2[\mathrm{Ohm}]$ \\
$L$ & $0.5[\mathrm{H}]$ \\
$k_{m}$ & $0.015[\mathrm{~N} . \mathrm{m} / \mathrm{A}]$ \\
$k_{f}$ & $0.02[\mathrm{~N} . \mathrm{m} . \mathrm{s}]$ \\
$k_{b}$ & $0.015[\mathrm{~V} / \mathrm{rad} / \mathrm{s}]$ \\
$\Gamma$ & $0.5[\mathrm{~N} . \mathrm{m}]$ \\
\hline \hline
\end{tabular}





\section{Appendix B}

\section{PhD Publications}

\section{B.1 Peer Reviewed Journal Papers}

\section{B.1.1 Published Journal Papers}

1. M. R. Amini, M. Shahbakhti, S. Pan, and J. K. Hedrick, "Bridging the Gap between Designed and Implemented Controllers via Adaptive Robust Discrete Sliding Mode Control," Journal of Control Engineering Practice, Vol. 59, Pages 1-15, February 2017. (Ref. [6])

2. M. R. Amini, M. Razmara, and M. Shahbakhti, "Robust Model-Based Discrete Sliding Mode Control of an Automotive Electronic Throttle Body", SAE International Journal of Commercial Vehicles, Vol. 10, No. 1, 14 Pages, 2017. (Ref. [9])

3. M. Shabakhti, M. R. Amini, J. Li, S. Asami, and J. K. Hedrick, "Early ModelBased Design and Verification of Automotive Control System Software Implementations," ASME Journal of Dynamic Systems, Measurement and Control, 
Vol. 137, Issue 2, 14 Pages, February 2015. (Ref. [1])

4. M. R. Amini, M. Shahbakhti, and A. Ghaffari, "A Novel Singular Perturbation Technique for Model-based Control of Cold Start Hydrocarbon Emission," SAE Int. Journal of Engines, Vol. 7, No. 3, Pages 1290-1301, 2014. (Ref. [2])

\section{B.1.2 Submitted Journal Paper}

1. M. R. Amini, M. Shahbakhti, S. Pan, and J. K. Hedrick, "Discrete MIMO Adaptive Second Order Sliding Mode Control of Uncertain Nonlinear Systems," submitted to IEEE Trans on Control Systems Technology, 15 pages, Jan 2017.

\section{B.2 Peer-Reviewed Conference Papers}

1. M. R. Amini, M. Razmara, and M. Shahbakhti, "Robust Model-Based Discrete Sliding Mode Control of an Automotive Electronic Throttle Body," SAE World Congress, 14 Pages, SAE Technical Paper 2017-01-0598, Detroit, MI, 2017. (Ref. [9])

2. M. R. Amini, M. Shahbakhti, S. Pan, and J. K. Hedrick, "Discrete Adaptive Second Order Sliding Mode Controller Design with Application to Automotive Control Systems with Model Uncertainties," American Control Conference (ACC), 6 Pages, Seattle, WA, 2017. (Ref. [8])

3. M. R. Amini, M. Shahbakhti, S. Pan, and J. K. Hedrick, "Handling Model and Implementation Uncertainties via an Adaptive Discrete Sliding Mode Controller 
Design," ASME Dynamic System and Control Conference (DSCC), 10 Pages, Minneapolis, MN, 2016. (Ref. [5])

4. M. R. Amini, M. Shahbakhti, and J. K. Hedrick, "Discrete Sliding Controller Design with Robustness to Implementation Imprecisions via Online Uncertainty Prediction," American Control Conference (ACC), 6 Pages, Boston, MA, 2016. (Ref. [4])

5. M. R. Amini, M. Shahbakhti, and J. K. Hedrick, "Easily Verifiable Adaptive Sliding Mode Controller Design with Application to Automotive Engines," SAE World Congress, 16 Pages, SAE Technical Paper 2016-01-0629, Detroit, MI, 2016. (Ref. [3])

6. M. R. Amini, M. Shahbakhti, and A. Ghaffari, "A Novel Singular Perturbation Technique for Model-based Control of Cold Start Hydrocarbon Emission," SAE World Congress, 13 Pages, SAE Technical Paper 2014-01-1547, Detroit, MI, 2014. (Ref. [2])

\section{B.2.1 Submitted Conference Papers}

1. M. R. Amini, M. Shahbakhti, S. Pan, and J. K. Hedrick, "MIMO First and Second Order Discrete Sliding Mode Controls of Uncertain Linear Systems Under Implementation Imprecisions," submitted to ASME Dynamic System and Control Conference (DSCC), 10 pages, Tysons Corner, VA, Feb. 2017. 
2. M. R. Amini and M. Shahbakhti, "Design of an SI Engine Cold Start Controller based on Dynamic Coupling Analysis," Abstract submitted to 2017 International Conference on Advanced Vehicle Powertrains, Hangzhou, China, Feb. 2017. 


\section{Appendix $\mathrm{C}$}

\section{Program and Data File Summary}

Following files were used for this dissertation arranged in the tables.

C.1 Chapter 1

Table C.1

Chapter 1 Figure files.

\begin{tabular}{cc|}
\hline \hline File name & File description \\
\hline Figure_1_1.vsd & Figure 1.1 \\
Figure_1_2.vsd & Figure $\overline{1.2}$ \\
Figure_1_3.vsd & Figure $\overline{\overline{1.3}}$ \\
Figure_1_4.jpeg & Figure $\overline{\overline{1.4}}$ \\
Figure_1_5.vsd & Figure $\overline{\overline{1.5}}$ \\
\hline \hline
\end{tabular}

C.2 Chapter 2

Table C.2

Chapter 2 Figure files.

\begin{tabular}{cc|c|}
\hline \hline File name & File description \\
\hline Figure_2_1.vsd & Figure & 2.1 \\
Figure_2_2.vsd & Figure & 2.2 \\
Figure_2_3.vsd & Figure & 2.3 \\
Figure_2_4.fig & Figure & 2.4 \\
Figure_2_5.fig & Figure & 2.5 \\
Figure_2_6.fig & Figure & 2.6 \\
Figure_2_7.vsd & Figure & 2.7 \\
Figure_2_8.fig & Figure & 2.8 \\
Figure_2_9.fig & Figure & 2.9 \\
Figure_2_10.fig & Figure & 2.10 \\
\hline \hline
\end{tabular}


Table C.3

Chapter 2 MATLAB script and SIMULINK files.

\begin{tabular}{cc}
\hline \hline File name & File description \\
\hline SPA_Reduced_Order_SMC.m & Reduced Order SMC \\
walker_filter.m & Walker Filter \\
load_singular_data2.m & Load exp data \\
balanced_realization_nonlinear.m & Nonlinear balanced realization \\
balanced_truncation.m & Balanced truncation \\
singular_perturbation.m & Singular perturbation \\
engine_model_nf_oneoutput.mdl & approximation \\
smc_4balrea_engmodel4.mdl & Engine model \\
\hline \hline
\end{tabular}

C.3 Chapter 3

Table C.4

Chapter 3 Figure files.

\begin{tabular}{|c|c|}
\hline File name & File description \\
\hline Figure_3_1.vsd & Figure 3.1 \\
\hline Figure_3_2_a.fig, Figure_3_2_b.fig, Figure_3_2_c.fig & Figure $\overline{\overline{3.2}}$ \\
\hline Figure_3_3.fig & Figure $\overline{3.3}$ \\
\hline Figure_3_4.fig & Figure 3.4 \\
\hline Figure_3_5.vsd & Figure 3.5 \\
\hline Figure_3_6.vsd & Figure 3.6 \\
\hline Figure_3_7_a.fig, Figure_3_7_b.fig, Figure_3_7_c.fig & Figure 3.7 \\
\hline Figure_3_8.fig & Figure $\overline{\overline{3.8}}$ \\
\hline Figure_3_9.fig & Figure $\overline{3.9}$ \\
\hline
\end{tabular}


Table C.5

Chapter 3 MATLAB script and SIMULINK files.

\begin{tabular}{cc}
\hline \hline File name & File description \\
\hline Adaptive_MIMO_SMC.m & Uncertainty adaptive \\
walker_filter.m & MIMO SMC \\
load_singular_data2.m & Walker Filter \\
balanced_realization_nonlinear.m & Nonlinear balanced realization \\
balanced_truncation.m & Balanced truncation \\
engine_model_nf_oneoutput.mdl & Engine model \\
smc_4balrea_engmodel3Orig.mdl & Engine Controller \\
linmod_engmodel3_nf.m & Linear engine model \\
\hline \hline
\end{tabular}

\section{C.4 Chapter 4}

Table C.6

Chapter 4 Figure files.

\begin{tabular}{|c|c|}
\hline File name & File descriptio \\
\hline Figure_4_1.vsd & Figure 4.1 \\
\hline Figure_4_2.vsd & Figure $\overline{\overline{4.2}}$ \\
\hline Figure_4_3.vsdx & Figure $\overline{\overline{4.3}}$ \\
\hline Figure_4_4.fig & Figure $\overline{4.4}$ \\
\hline Figure_4_5.fig & Figure $\overline{4.5}$ \\
\hline Figure_4_6_a.fig, Figure_4_6_b.fig, Figure_4_6_c.fig & Figure $\overline{4.6}$ \\
\hline Figure_4_7.fig & Figure 4.7 \\
\hline Figure_4_8.fig & Figure $\overline{\overline{4.8}}$ \\
\hline
\end{tabular}

Table C.7

Chapter 4 MATLAB script and SIMULINK files (Engine case study).

\begin{tabular}{cc}
\hline \hline File name & File description \\
\hline Engine_First_Order_DSMC_Robust.m & Robust First Order \\
walker_filter.m & SISO DSMC \\
load_trajectories.m & Walker Filter \\
dsmc_ADC2.mdl & Load desired \\
\hline \hline
\end{tabular}


Table C.8

Chapter 4 MATLAB script and SIMULINK files (DC motor case study).

\begin{tabular}{cc}
\hline \hline File name & File description \\
\hline DC_Motor_First_Order_DSMC.m & Robust First Order \\
dS_MRAC_ADC_DSMC.mdl & SISO DSMC \\
\hline \hline
\end{tabular}

\section{C.5 Chapter 5}

Table C.9

Chapter 5 Figure files.

\begin{tabular}{|c|c|c|}
\hline File name & File desc & criptic \\
\hline Figure_5_1.vsd & Figure & e 5.1 \\
\hline Figure_5_2.vsd & Figure & $\mathrm{e} \overline{5.2}$ \\
\hline Figure_5_3.fig & Figure & $\mathrm{e} 5.3$ \\
\hline Figure_5_4.fig & Figure & $\mathrm{e} \overline{5.4}$ \\
\hline Figure_5_5.fig & Figure & $\mathrm{e} 5.5$ \\
\hline Figure_5_6.fig & Figure & $\mathrm{e} 5.6$ \\
\hline Figure_5_7.fig & Figure & $\mathrm{e} 5.7$ \\
\hline Figure_5_8.fig & Figure & $\mathrm{e} \overline{5.8}$ \\
\hline Figure_5_9.fig & Figure & $\mathrm{e} \overline{5.9}$ \\
\hline Figure_5_10.fig & Figure & 5.10 \\
\hline Figure_5_11.fig & Figure & 5.11 \\
\hline Figure_5_12.fig & Figure & 5.12 \\
\hline Figure_5_13.fig & Figure & 5.13 \\
\hline
\end{tabular}

Table C.10

Chapter 5 MATLAB script and SIMULINK files (Engine case study).

\begin{tabular}{cc}
\hline \hline File name & File description \\
\hline Engine_SISO_Adaptive_DSMC.m & Adaptive Robust First \\
& Order SISO DSMC \\
walker_filter.m & Walker Filter \\
load_trajectories.m & Load desired \\
& engine trajectories \\
dsmc_ADC_Adaptive_combine.mdl & Engine Controller \\
\hline \hline
\end{tabular}


Table C.11

Chapter 5 MATLAB script and SIMULINK files (DC motor case study).

\begin{tabular}{cc}
\hline \hline File name & File description \\
\hline DC_Motor_Adaptive_DSMC.m & Adaptive Robust First \\
DC_Motor_Adaptive_DSMC_Sim.mdl & Order SISO DSMC \\
\hline \hline
\end{tabular}

\section{C.6 Chapter 6}

Table C.12

Chapter 6 Figure files.

\begin{tabular}{cc|c|}
\hline \hline File name & File description \\
\hline Figure_6_1.fig & Figure & 6.1 \\
Figure_6_2.fig & Figure & $\overline{6.2}$ \\
Figure_6_3.fig & Figure & $\overline{6.3}$ \\
Figure_6_4.fig & Figure & $\overline{6.4}$ \\
Figure_6_5.fig & Figure & $\overline{6.5}$ \\
Figure_6_6.fig & Figure & $\overline{6.6}$ \\
Figure_6_7.fig & Figure & $\overline{6.7}$ \\
Figure_6_8.fig & Figure & $\overline{6.8}$ \\
Figure_6_9.fig & Figure & 6.9 \\
Figure_6_10.fig & Figure & 6.10 \\
Figure_6_11.fig & Figure & 6.11 \\
Figure_6_12.fig & Figure & 6.12 \\
Figure_6_13.fig & Figure & 6.13 \\
\hline \hline
\end{tabular}

Table C.13

Chapter 6 MATLAB script and SIMULINK files (Engine case study).

\begin{tabular}{cc}
\hline \hline File name & File description \\
\hline Engine_MIMO_Second_Order_DSMC.m & Adaptive Robust Second \\
walker_filter.m & Order MIMO DSMC \\
load_trajectories.m & Walker Filter \\
MIMO2OrderDSMC.mdl & Load desired \\
engine trajectories \\
\hline \hline
\end{tabular}


Table C.14

Chapter 6 MATLAB script and SIMULINK files (DC motor case study).

\begin{tabular}{cc}
\hline \hline File name & File description \\
\hline DC_Motor_MIMO_Second_Order_DSMC.m & Adaptive Second \\
Order MIMO DSMC \\
DC_2DSMC_Final.mdl & DC motor Controller \\
\hline \hline
\end{tabular}

\section{C.7 Chapter 7}

Table C.15

Chapter 7 Figure files.

\begin{tabular}{|c|c|c|}
\hline File name & File descr & ription \\
\hline Figure_7_1.vsd & Figure & 7.1 \\
\hline Figure_7_2.vsd & Figure & $\overline{7.2}$ \\
\hline Figure_7_3.vsd & Figure & e \\
\hline Figure_7_4.fig & Figure & 7.4 \\
\hline Figure_7_5.fig & Figure & 7.5 \\
\hline Figure_7_6.fig & Figure & 7.6 \\
\hline Figure_7_7.fig & Figure & 7.7 \\
\hline Figure_7_8.fig & Figure & 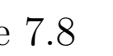 \\
\hline Figure_7_9.fig & Figure & 7.9 \\
\hline Figure_7_10.fig & Figure & 7.10 \\
\hline Figure_7_11.fig & Figure & 7.11 \\
\hline Figure_7_12.fig & Figure & 7.12 \\
\hline Figure_7_13.fig & Figure & 7.13 \\
\hline Figure_7_14.fig & Figure & 7.14 \\
\hline Figure_7_15.fig & Figure & 7.15 \\
\hline
\end{tabular}


Table C.16

Chapter 7 MATLAB script, SIMULINK files, and experimental data.

\begin{tabular}{cc}
\hline \hline File name & File description \\
\hline DSMC_code.m & Throttle Robust \\
DDSMC_V2.mdl & SISO DSMC \\
Throttle_model_ident.m & Throttle model identification \\
ident_U_sim.mat & Control input exp data (1) \\
ident_U_sim_2.mat & Control input exp data (2) \\
ident_TPS_sim.mat & TPS exp data (1) \\
ident_TPS_sim_2.mat & TPS exp data (2) \\
filtering_data.mdl & Digital low pass filter \\
ident new 04012016 & Folder of throttle \\
exp1_001.csv & identification data \\
exp1_002.csv & Experimental DSMC test data (1) \\
exp1_003.csv & Experimental DSMC test data (2) \\
\hline \hline
\end{tabular}



Appendix D

\section{Letters of Permission}




\section{D.1 Letter of Permission for Ref [1] (Chapter 2)}

M. Reza Amini <mamini@mtu.edu>

Permission Request for J. Dyn. Sys., Meas., Control 137(2), 021006

Beth Darchi <DarchiB@asme.org>

Wed, Mar 1, 2017 at 4:15 PM

To: "M. Reza Amini" <mamini@mtu.edu>

Dear Prof. Amini,

It is our pleasure to grant you permission to use all or any part of the ASME paper "Early Model-Based Design and Verification of Automotive Control System Software Implementations," by Mahdi Shahbakhti1, Mohammad Reza Amini, Jimmy Li, Satoshi Asami and J. Karl Hedrick, J. Dyn. Sys., Meas., Control 137(2), 2014, cited in your letter for inclusion in a PhD dissertation entitled Easily verifiable controller design with application to automotive powertrains to be published by Michigan Technological University.

Permission is granted for the specific use as stated herein and does not permit further use of the materials without proper authorization. Proper attribution must be made to the author(s) of the materials. Please note: if any or all of the figures and/or Tables are of another source, permission should be granted from that outside source or include the reference of the original source. ASME does not grant permission for outside source material that may be referenced in the ASME works.

As is customary, we request that you ensure full acknowledgment of this material, the author(s), source and ASME as original publisher. Acknowledgment must be retained on all pages where figure is printed and distributed.

Many thanks for your interest in ASME publications

Sincerely,

Beth Darchi

Publishing Administrator

ASME

2 Park Avenue

New York, NY 10016-5990

Tel 1.212 .591 .7700

darchib@asme.org

Figure D.1: Letter of Permission for Ref [1]. 


\section{D.2 Letter of Permission for Ref [2] (Chapter 2)}

Michigan Tech

Permission Request for SAE Paper 2014-01-1547

copyright <copyright@sae.org>

To: "M. Reza Amini" <mamini@mtu.edu>, copyright <copyright@sae.org>

Wed, Mar 15, 2017 at 1:08 PM

Dear Reza,

Thank you for your request.

Please be advised, reproduction use of SAE Technical Paper 2014-01-1547, 2014, is hereby granted, and subject to the following terms and conditions:

-Permission is granted for non-exclusive English language rights, and for the specific use as indicated in your email;

-A new Copyright Permission Request is required for any further use of this material, other than that specified within your original Request;

-The SAE material must be clearly identified and include the following statement "Reprinted with permission by SAE (C) 2017 SAE International. Further distribution of this material is not permitted without prior permission from SAE.";

-We also request that you include a complete reference to the SAE document in the reference section for each figure used;

-Any language use, other than specified within your email, requires a new copyright request and additional fee;

-This permission does not cover any third-party copyrighted work which may appear in the material requested;

-Licensor's use of this material, in whole or in part, is entirely its responsibility, and SAE International does not warrant or is not responsible for any use of the material.

Thank you,

Nikole Aston

IP Compliance Specialist

SAE INTERNATIONAL

400 Commonwealth Drive

Warrendale, PA 15096 USA

o: +1.724 .772 .4095$

e: nikole.aston@sae.org

www.sae.org

Figure D.2: Letter of Permission for Ref [2]. 


\section{D.3 Letter of Permission for Ref [3] (Chapter 3)}

Michigan Tech

\section{Permission Request for SAE Paper 2016-01-0629}

copyright <copyright@sae.org>

To: "M. Reza Amini" <mamini@mtu.edu>, copyright <copyright@sae.org>

Wed, Mar 15, 2017 at 12:13 PM

Dear Reza,

Thank you for your request.

Please be advised, reproduction use of SAE Technical Paper 2016-01-0629, 2016, is hereby granted, and subject to the following terms and conditions:

-Permission is granted for non-exclusive English language rights, and for the specific use as indicated in your email;

-A new Copyright Permission Request is required for any further use of this material, other than that specified within your original Request;

-The SAE material must be clearly identified and include the following statement "Reprinted with permission by SAE (C) 2017 SAE International. Further distribution of this material is not permitted without prior permission from SAE.";

-We also request that you include a complete reference to the SAE document in the reference section for each figure used;

-Any language use, other than specified within your email, requires a new copyright request and additional fee;

-This permission does not cover any third-party copyrighted work which may appear in the material requested;

-Licensor's use of this material, in whole or in part, is entirely its responsibility, and SAE International does not warrant or is not responsible for any use of the material.

Thank you,

Nikole Aston

IP Compliance Specialist

SAE INTERNATIONAL

400 Commonwealth Drive

Warrendale, PA 15096 USA

o: +1.724 .772 .4095$

e: nikole.aston@sae.org

www.sae.org

Figure D.3: Letter of Permission for Ref [3]. 


\section{D.4 Letter of Permission for Ref [4] (Chapter 4)}

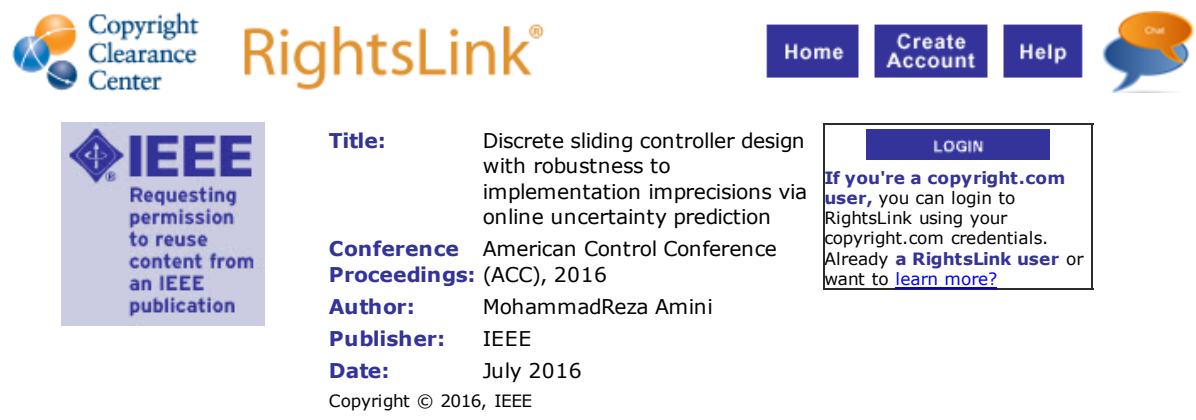

Thesis / Dissertation Reuse

The IEEE does not require individuals working on a thesis to obtain a formal reuse license, however, you may print out this statement to be used as a permission grant:

Requirements to be followed when using any portion (e.g., figure, graph, table, or textual material) of an IEEE copyrighted paper in a thesis:

1) In the case of textual material (e.g., using short quotes or referring to the work within these papers) users must give full credit to the original source (author, paper, publication) followed by the IEEE copyright line 2011 IEEE.

2 ) In the case of illustrations or tabular material, we require that the copyright line [Year of original publication] IEEE appear prominently with each reprinted figure and/or table.

3) If a substantial portion of the original paper is to be used, and if you are not the senior author, also obtain the senior author $\gg$ approval.

Requirements to be followed when using an entire IEEE copyrighted paper in a thesis:

1) The following IEEE copyright/ credit notice should be placed prominently in the references: original publication] IEEE. Reprinted, with permission, from [author names, paper title, IEEE publication title, and month/year of publication]

2) Only the accepted version of an IEEE copyrighted paper can be used when posting the paper or your thesis on-line.

3) In placing the thesis on the author's university website, please display the following message in a prominent place on the website: In reference to IEEE copyrighted material which is used with permission in this thesis, the IEEE does not endorse any of [university/educational entity's name goes here]'s products or services. Internal or personal use of this material is permitted. If interested in reprinting/republishing IEEE copyrighted material for advertising or promotional purposes or for creating new collective works for resale or redistribution, please go to

http://www.ieee.org/publications standards/publications/rights/rights link.html to learn how to obtain a License from RightsLink.

If applicable, University Microfilms and/or ProQuest Library, or the Archives of Canada may supply single copies of the dissertation.

\section{BACK \\ CLOSE WINDOW}

Copyright ( $) 2017$ Copyright Clearance Center, Inc. All Rights Reserved. Privacy statement. Terms and Conditions. Comments? We would like to hear from you. E-mail us at customercare@copyright.com

Figure D.4: Letter of Permission for Ref [4]. 


\section{D.5 Letter of Permission for Ref [5] (Chapter 5)}

M. Reza Amini <mamini@mtu.edu>

Permission Request for ASME 2016 Dynamic Systems and Control Conference, Paper No. DSCC2016-9732

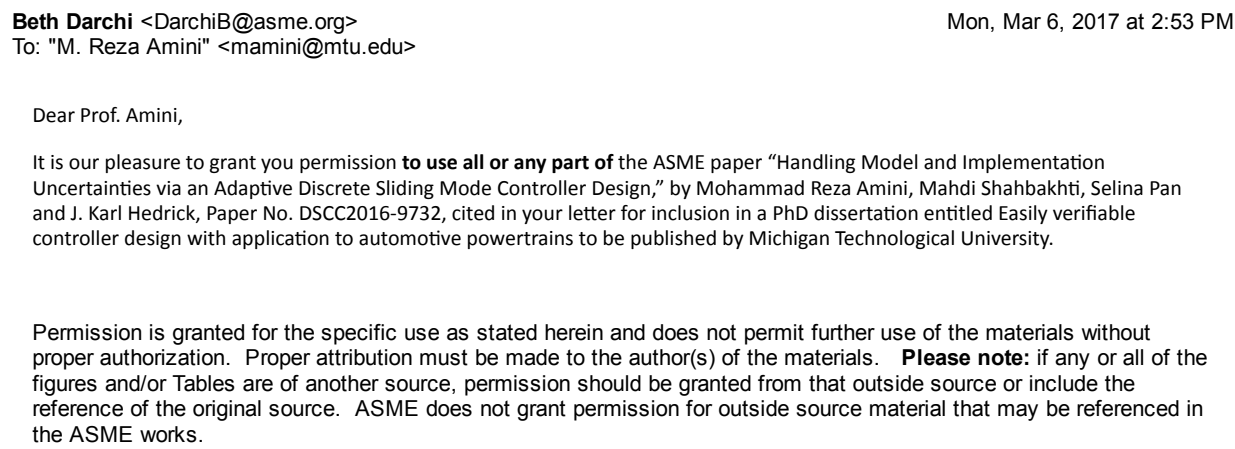

It is our pleasure to grant you permission to use all or any part of the ASME paper "Handling Model and Implementation Uncertainties via an Adaptive Discrete Sliding Mode Controller Design," by Mohammad Reza Amini, Mahdi Shahbakhti, Selina Pan and J. Karl Hedrick, Paper No. DSCC2016-9732, cited in your letter for inclusion in a PhD dissertation entitled Easily verifiable controller design with application to automotive powertrains to be published by Michigan Technological University.

Permission is granted for the specific use as stated herein and does not permit further use of the materials without proper authorization. Proper attribution must be made to the author(s) of the materials. Please note: if any or all of the figures and/or Tables are of another source, permission should be granted from that outside source or include the reference of the original source. ASME does not grant permission for outside source material that may be referenced in the ASME works.

As is customary, we request that you ensure full acknowledgment of this material, the author(s), source and ASME as original publisher. Acknowledgment must be retained on all pages where figure is printed and distributed.

Many thanks for your interest in ASME publications.

Sincerely,

Beth Darchi

Publishing Administrator

ASME

2 Park Avenue

New York, NY 10016-5990

Tel 1.212 .591 .7700

darchib@asme.org

Figure D.5: Letter of Permission for Ref [5]. 


\section{D.6 Letter of Permission for Ref [6] (Chapter 5)}

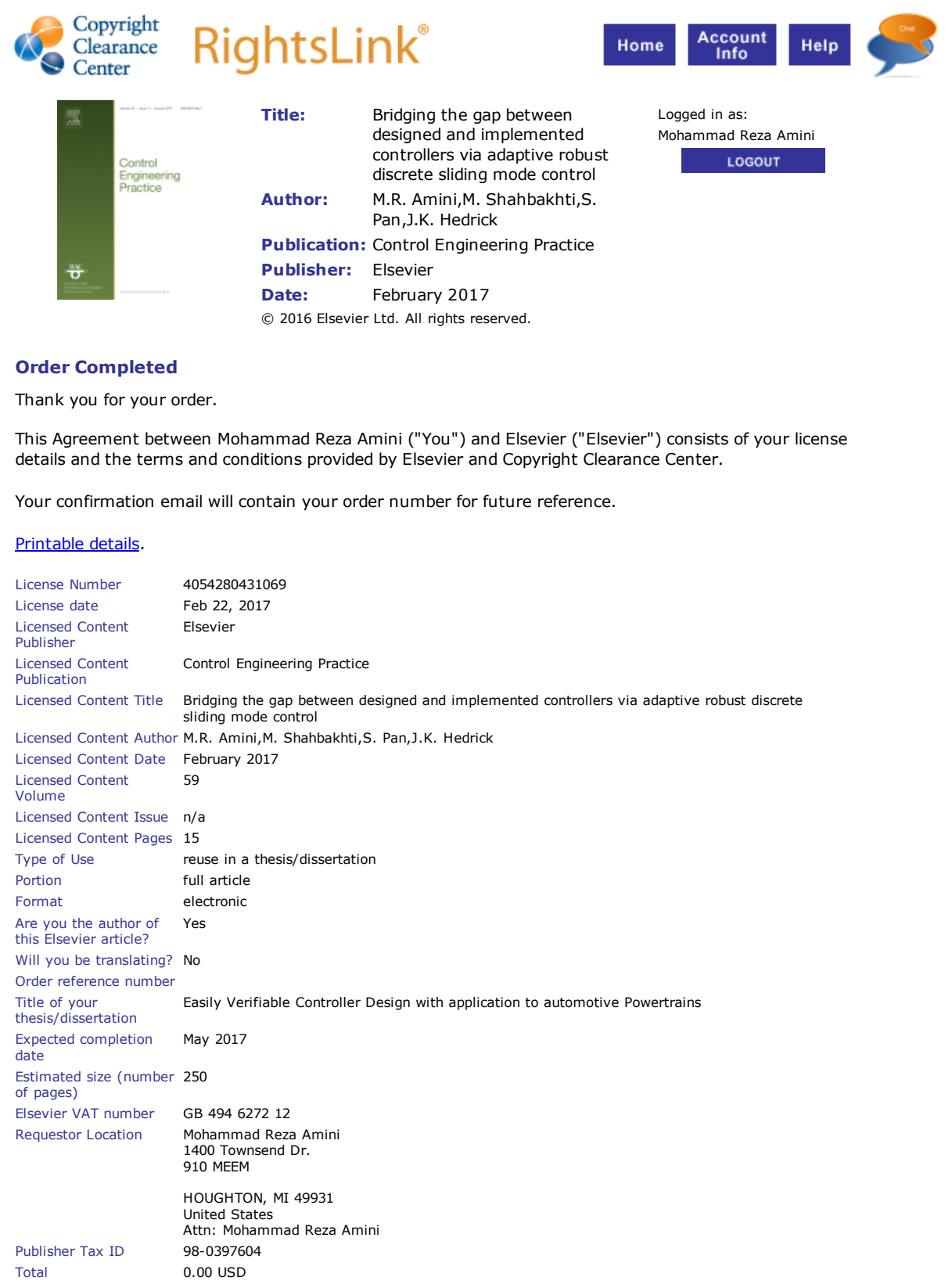

Figure D.6: Letter of Permission for Ref [6]. 


\title{
D.7 Letter of Permission for Ref [9] (Chapter 7)
}

M. Reza Amini <mamini@mtu.edu>

\author{
Permission Request for SAE Paper 2017-01-0598 \\ copyright <copyright@sae.org> \\ To: "M. Reza Amini" <mamini@mtu.edu>, copyright <copyright@sae.org> \\ Fri, May 5, 2017 at 3:59 PM \\ Dear Reza, \\ Please be advised, reproduction use of SAE Paper 2017-01-0598, is hereby granted, and subject to the following terms \\ and conditions: \\ -Permission is granted for non-exclusive English language rights, and for the specific use as indicated in your email; \\ -A new Copyright Permission Request is required for any further use of this material, other than that specified within \\ your original Request; \\ -The SAE material must be clearly identified and include the following statement "Reprinted with permission by SAE \\ (C) 2017 SAE International. Further distribution of this material is not permitted without prior permission from SAE."; \\ -We also request that you include a complete reference to the SAE document in the reference section for each figure \\ used; \\ -Any language use, other than specified within your email, requires a new copyright request and additional fee; \\ -This permission does not cover any third-party copyrighted work which may appear in the material requested; \\ -Licensor's use of this material, in whole or in part, is entirely its responsibility, and SAE International does not \\ warrant or is not responsible for any use of the material. \\ Thank you, \\ Nikole Aston \\ IP Compliance Specialist \\ SAE INTERNATIONAL \\ 400 Commonwealth Drive \\ Warrendale, PA 15096 USA \\ o: +1.724 .772 .4095$ \\ e: nikole.aston@sae.org \\ www.sae.org \\ From: M. Reza Amini [mailto:mamini@mtu.edu] \\ Sent: Friday, May 05, 2017 12:49 PM \\ To: copyright <copyright@sae.org> \\ Subject: Permission Request for SAE Paper 2017-01-0598
}

Figure D.7: Letter of Permission for Ref [9]. 


\section{D.8 Letter of Permission for Figure 2.4 [7]}

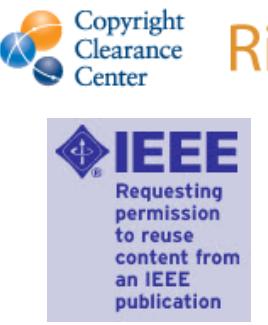

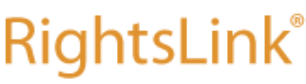

Title:

\section{Hybrid switching control of} automotive cold start hydrocarbon emission

Conference American Control Conference

Proceedings: (ACC), 2013

Author: Rasoul Salehi

Publisher: IEEE

Date: June 2013

Copyright @) 2013, IEEE

\section{Home $\underset{\substack{\text { Create } \\ \text { Account }}}{\text { Help }}$}

LOGIN

user, you can login to

RightsLink using your
Ris.

copyright.com credentials.

Already a RightsLink user or want to learn more?

\section{Thesis / Dissertation Reuse}

The IEEE does not require individuals working on a thesis to obtain a formal reuse license, however, you may print out this statement to be used as a permission grant:

Requirements to be followed when using any portion (e.g., figure, graph, table, or textual material) of an IEEE copyrighted paper in a thesis:

1) In the case of textual material (e.g., using short quotes or referring to the work within these papers) users must give full credit to the original source (author, paper, publication) followed by the IEEE copyright line 2011 IEEE.

2 ) In the case of illustrations or tabular material, we require that the copyright line [Year of original publication] IEEE appear prominently with each reprinted figure and/or table.

3) If a substantial portion of the original paper is to be used, and if you are not the senior author, also obtain the senior author $\$$ s approval.

Requirements to be followed when using an entire IEEE copyrighted paper in a thesis:

1) The following IEEE copyright/ credit notice should be placed prominently in the references: original publication] IEEE. Reprinted, with permission, from [author names, paper title, IEEE publication title, and month/year of publication]

2) Only the accepted version of an IEEE copyrighted paper can be used when posting the paper or your thesis on-line.

3) In placing the thesis on the author's university website, please display the following message in a prominent place on the website: In reference to IEEE copyrighted material which is used with permission in this thesis, the IEEE does not endorse any of [university/educational entity's name goes here]'s

products or services. Internal or personal use of this material is permitted. If interested in

reprinting/republishing IEEE copyrighted material for advertising or promotional purposes or for creating new collective works for resale or redistribution, please go to

http://www.ieee.org/publications standards/publications/rights/rights link.html to learn how to obtain a License from RightsLink.

If applicable, University Microfilms and/or ProQuest Library, or the Archives of Canada may supply single copies of the dissertation.

Figure D.8: Letter of Permission for Figure 2.4 [7]. 